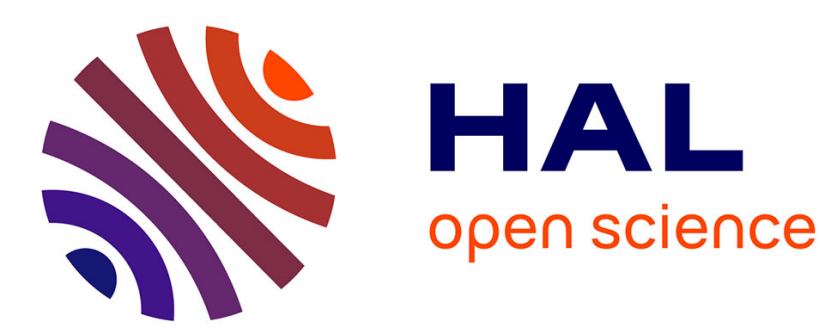

\title{
SYMMETRIC SPACES AND THE KASHIWARA-VERGNE METHOD
}

François Rouvière

\section{To cite this version:}

François Rouvière. SYMMETRIC SPACES AND THE KASHIWARA-VERGNE METHOD. 2014, 10.1007/978-3-319-09773-2 . hal-01331776

\section{HAL Id: hal-01331776 \\ https://hal.univ-cotedazur.fr/hal-01331776}

Submitted on 14 Jun 2016

HAL is a multi-disciplinary open access archive for the deposit and dissemination of scientific research documents, whether they are published or not. The documents may come from teaching and research institutions in France or abroad, or from public or private research centers.
L'archive ouverte pluridisciplinaire HAL, est destinée au dépôt et à la diffusion de documents scientifiques de niveau recherche, publiés ou non, émanant des établissements d'enseignement et de recherche français ou étrangers, des laboratoires publics ou privés. 


\section{SYMMETRIC SPACES AND THE KASHIWARA-VERGNE METHOD}

François Rouvière

June 30, 2014 


\section{Contents}

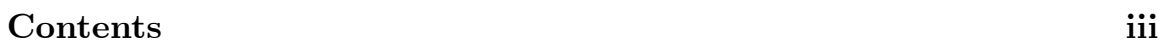

Abstract vii

Preface $\quad$ ix

Introduction $\quad x i$

Notation $\quad$ xv

0.1 General Notation . . . . . . . . . . . . . . . xv

0.2 Lie Groups and Lie Algebras . . . . . . . . . . . . . . . xvi

0.3 Symmetric Lie Algebras . . . . . . . . . . . . . . . . . . xvi

0.4 Symmetric Spaces . . . . . . . . . . . . . . . . . xvii

0.5 Semisimple Notation . . . . . . . . . . . . . . . . xviii

1 Kashiwara-Vergne Method for Lie groups 1

1.1 A General Convolution Problem . . . . . . . . . . . . . 1

1.2 The Kashiwara-Vergne Method . . . . . . . . . . . . . . . 3

1.3 The Equation $(\mathrm{KV} 1) \ldots \ldots \ldots \ldots$

1.4 The Equation $(\mathrm{KV} 2) \ldots \ldots \ldots \ldots$

1.5 The Kashiwara-Vergne Conjecture . . . . . . . . . . . . . 10

1.5.1 The Conjecture . . . . . . . . . . . . . . . 10

1.5.2 Remarks on the Conjecture . . . . . . . . . . . . 11

1.5.3 Non-Uniqueness . . . . . . . . . . . . . . . . . . 14

$1.6 \quad$ An Elementary Proof for $s l(2, \mathbb{R}) \ldots \ldots \ldots \ldots$

1.6.1 Choice of $F$ and $G \ldots \ldots \ldots \ldots \ldots$

1.6.2 A Variant of $(\mathrm{KV} 2) \ldots \ldots \ldots \ldots$

1.6.3 A Trace Formula for $s l(2, \mathbb{R}) \ldots \ldots \ldots \ldots$

1.6.4 Proof of $(\mathrm{KV} 2) \ldots \ldots \ldots \ldots$

1.7 Two Symmetries . . . . . . . . . . . . . . . . . . . . . 20

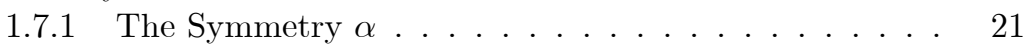

1.7.2 The Symmetry $\beta \ldots \ldots \ldots$. . . . . . . . . . . 21

1.7.3 Symmetry Invariant Solutions . . . . . . . . . . . . 24

1.8 From (KV1) Towards $(\mathrm{KV} 2) \ldots \ldots \ldots \ldots$ 
1.8.1 Tangential Derivations and Automorphisms . . . . . 26

1.8.2 Behavior Under Scaling Maps . . . . . . . . . . . . . 28

1.8.3 Using Associativity . . . . . . . . . . . . . . . . 29

1.8.4 A «Divergence» and a «Jacobian» . . . . . . . . . . 30

1.8.5 A Cohomology Argument . . . . . . . . . . . . 33

1.8.6 An Expression of the Divergence . . . . . . . . . . 34

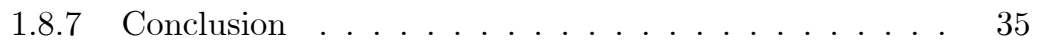

1.9 Quadratic or Solvable Lie Algebras . . . . . . . . . . . . . . . 38

1.10 The General Case . . . . . . . . . . . . . . . . . . . . . . 42

1.10.1 An Abstract Formulation of the Kashiwara-Vergne Problem..................... 42

1.10.2 Drinfeld's Associators and the Kashiwara-Vergne Con-

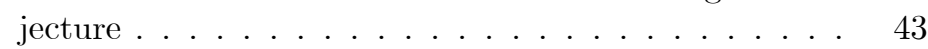

Notes .............................. 44

2 Convolution on Homogeneous Spaces $\quad 47$

2.1 Definition and Examples . . . . . . . . . . . . . . . . . 47

2.2 Extension to Line Bundles . . . . . . . . . . . . . . . . . . . 50

2.2.1 The Line Bundle $L_{\chi} \ldots \ldots \ldots$. . . . . . . . . . . . 50

2.2.2 Convolution of Distributions on $L_{\chi} \ldots \ldots \ldots$. . . . 51

Notes . . . . . . . . . . . . . . . . . 51

3 The Role of $e$-Functions 53

3.1 Transferring Convolutions ............... 54

3.1.1 The Transfer Map . . . . . . . . . . . . . . 54

3.1 .2 e-Functions . . . . . . . . . . . . . . . . . 56

3.2 Special Symmetric Spaces . . . . . . . . . . . . . . . . 59

3.3 Invariant Differential Operators . . . . . . . . . . . . . 63

3.3.1 The Fischer Product . . . . . . . . . . . . . . . 64

3.3.2 Explicit Transfer of Invariant Differential Operators . 65

3.3.3 Example: the Laplace-Beltrami Operator . . . . . . . 68

3.3.4 Towards a Generalized Duflo Isomorphism . . . . . . . 71

3.4 Mean Values and Spherical Functions . . . . . . . . . . . . 73

3.4.1 The Case of an Isotropic Riemannian Symmetric Space 73

3.4.2 Expansion of Mean Value Operators and Spherical Functions .................. . . 76

3.5 Integral Formulas . . . . . . . . . . . . . . . . . . . . . . . . 80

3.6 Noncompact Symmetric Spaces . . . . . . . . . . . . . . 84

3.6.1 The Function $e_{\infty} \ldots \ldots \ldots \ldots$. . . . . . . 85

3.6.2 Link with the Iwasawa Projection . . . . . . . . . 89

3.6.3 Extension of Duflo's Isomorphism . . . . . . . . . . . 92

3.7 Symmetric Spaces of Rank One . . . . . . . . . . . . . . . . . 98

3.7.1 Outline of the Proof . . . . . . . . . . . . . . 99

3.7.2 Properties of the Rank One $e$-Function . . . . . . . . . 101

3.7.3 Application to Spherical Functions . . . . . . . . . . . 104 
3.8 Extension to Line Bundles . . . . . . . . . . . . . . . . . . 105

3.9 Open Problems . . . . . . . . . . . . . . . . . . . . 107

Notes . . . . . . . . . . . . . . . . . . . . . . . . . . 108

4 Campbell-Hausdorff $e$-Functions 111

4.1 Contractions of Symmetric Spaces . . . . . . . . . . . . 112

4.2 The Diffeomorphism $\Phi \ldots \ldots \ldots \ldots \ldots$

4.2 .1 Lie Series . . . . . . . . . . . . . . . . . . . . . 114

$4.2 .2 \quad \mathcal{O}, Z, h \ldots \ldots \ldots \ldots \ldots \ldots \ldots$

$4.2 .3 \quad A, B, C \ldots \ldots \ldots \ldots \ldots \ldots \ldots \ldots$

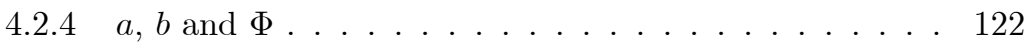

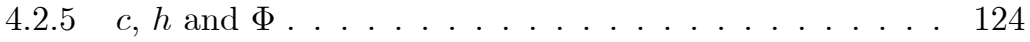

4.3 Campbell-Hausdorff $e$-Functions . . . . . . . . . . . 128

4.3.1 An Auxiliary Lemma . . . . . . . . . . . . . . . . 128

4.3.2 Construction of an e-Function . . . . . . . . . . . 129

4.3.3 Application to Invariant Differential Operators . . . . 133

4.4 Properties . . . . . . . . . . . . . . . . . 136

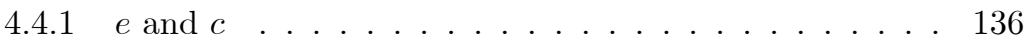

4.4.2 Some Deeper Properties of $e$. . . . . . . . . 137

4.5 Extension to Line Bundles . . . . . . . . . . . . . . . . . 146

4.6 Taylor Expansions . . . . . . . . . . . . . . . . . . . . . . . . 149

4.6.1 Expansions of $Z, h, A, B, C \ldots \ldots \ldots \ldots$

4.6 .2 Expansion of $\Phi \ldots \ldots \ldots \ldots \ldots \ldots$

4.6.3 Expansions of $e$ and $e_{\chi} \ldots \ldots \ldots \ldots \ldots$

4.6.4 Expansions of $Z, h, A, C$ Modulo $Y^{2} \ldots \ldots \ldots$

4.6.5 Expansion of $\Phi$ Modulo $Y^{2} \ldots \ldots \ldots$. . . . . . . 158

4.6.6 Expansions of $e$ and $e_{\chi}$ Modulo $Y^{2} \ldots \ldots \ldots \ldots$

4.7 Open Problems . . . . . . . . . . . . . . . . . . . 161

Notes . . . . . . . . . . . . . . . . . . . . 162

A Proof of Lemma 3.22 165

B Proof of Theorem $3.23 \quad 169$

$\begin{array}{ll}\text { Bibliography } & 179\end{array}$

$\begin{array}{ll}\text { Index } & 184\end{array}$ 


\begin{abstract}
The Kashiwara-Vergne method reduces the proof of a deep result in analysis on a Lie group (transferring convolution of invariant distributions from the group to its Lie algebra, by means of the exponential mapping) to checking two formal Lie brackets identities linked to the Campbell-Hausdorff formula. First, we expound this method and a proof, for quadratic or solvable Lie algebras, of the Kashiwara-Vergne conjecture allowing to apply the method to those cases.

We then extend it to a general symmetric space $S=G / H$. This leads to introduce a function $e(X, Y)$ of two tangent vectors $X, Y$ at the origin of $S$, allowing to make explicit, in the exponential chart, $G$-invariant differential operators of $S$, the structure of the algebra of all such operators, and an expansion of mean value operators and spherical functions. For Riemannian symmetric spaces of the noncompact type, otherwise well-known from the work of Harish-Chandra and Helgason, we compare this approach with the classical one. For rank one spaces (the hyperbolic spaces), we give an explicit formula for $e(X, Y)$.

Finally, we explain a construction of $e$ for a general symmetric space by means of Lie series linked to the Campbell-Hausdorff formula, in the spirit of the original Kashiwara-Vergne method. Proved from this construction, the main properties of $e$ thus link the fundamental tools of $H$-invariant analysis on a symmetric space to its infinitesimal structure.

The results extend to line bundles over symmetric spaces.
\end{abstract}

Mathematics Subject Classification (2010): 43A85, 53C35, 17B01, 43A90, 58J70, 22E30, 33C80.

Keywords: symmetric space, invariant differential operator, KashiwaraVergne conjecture, Duflo isomorphism, Campbell-Hausdorff formula.

François Rouvière

Laboratoire J. A. Dieudonné, UMR 7351 du CNRS

Université de Nice, Parc Valrose, 06108 Nice cedex 2, France

frou@unice.fr

http://math.unice.fr $/ \sim$ frou/ 


\section{Preface}

Let $G / K$ denote a Riemannian symmetric space of the noncompact type.

"The action of the $G$-invariant differential operators on $G / K$ on the radial functions on $G / K$ is isomorphic with the action of certain differential operators with constant coefficients. The isomorphism in question is used in Harish-Chandra's work on the Fourier analysis on $G$ and is related to the Radon transform on $G / K$.

For the case when $G$ is complex a more direct isomorphism of this type is given, again as a consequence of results of HarishChandra.»

This is a quote from Sigurður Helgason, Fundamental solutions of invariant differential operators on symmetric spaces, Amer. J. Maths. 86 (1964), p. 566 , one of the first mathematical papers I have studied. As a consequence he showed that every (non-zero) $G$-invariant differential operator $D$ on $G / K$ has a fundamental solution, whence the solvability of the differential equation $D u=f$. Those results were fascinating to me, they still are and they fostered my interest in invariant differential operators as well as Radon transforms. Yet I was dreaming simpler proofs could be given, without relying on HarishChandra's deep study of semisimple Lie groups...

In the beginning of 1977 Michel Duflo [18] gave an analytic interpretation of the celebrated Duflo isomorphism he had exhibited six years before by means of algebraic constructions in an enveloping algebra. As a consequence he proved that every (non-zero) bi-invariant differential operator on a Lie group has a local fundamental solution and is locally solvable. His work used delicate analysis on the group.

Then, in the fall of 1977, came a preprint by Masaki Kashiwara and Michèle Vergne, The Campbell-Hausdorff formula and invariant hyperfunctions [30], showing that similar (and even stronger) results could be obtained - for solvable Lie groups at least - by means of «elementary», but very clever, formal computations with the exponential mapping and the CampbellHausdorff formula only.

After explaining this method for Lie groups (with updates from the 200809 papers by Anton Alekseev and Charles Torossian) I will give here a detailed 
account of its extension to general symmetric spaces. The present text is an attempt at a self-contained monograph on the Kashiwara-Vergne approach to invariant analysis. A completely rewritten and updated version of my former papers [43] - [47], it includes several unpublished results and a few open questions. It is also a greatly expanded version of a talk given at the 2007 Reykjavik conference in honour of Helgason's eightieth birthday.

\section{Acknowledgement}

Sigurður Helgason's influence on my own work goes far beyond the above quote of course. His clearly written books and articles have been a constant reference and source of inspiration to me for many years and, above all, his friendly advice on many occasions has been extremely helpful. Thank you so much Sigurður.

I am indebted to Mogens Flensted-Jensen for several stimulating discussions, particularly about the rank one case.

I am very thankful to Anton Alekseev, Charles Torossian and Michèle Vergne for enlightening explanations of their work. 


\section{Introduction}

The 1978 Inventiones paper [30] by Kashiwara and Vergne was a breakthrough in the field of analysis on Lie groups. Before it, the proof of several significant results (notably results involving invariant differential operators, their local solvability, Duflo's isomorphism,...) required a detailed knowledge of the structure of the group and its Lie algebra, the use of specific subgroups etc. Their work opened a new way, only using skillful formal manipulations of Lie brackets, thus providing a direct link to the infinitesimal structure of the group. They carried out their program for solvable Lie groups and conjectured two general identities allowing an extension of the method to arbitrary Lie groups.

Two natural questions (at least) arose from their paper:

- prove the Kashiwara-Vergne conjecture, implying that their method is actually valid for all Lie groups

- investigate possible extensions of the method to symmetric spaces.

A complete solution to the former question kept us waiting for nearly thirty years. The conjecture is now a theorem, proved in full generality by two different methods in [5] resp. [7], by Alekseev and Meinrenken resp. Alekseev and Torossian; see the Notes of Chapter 1 for more details.

The latter question is the main topic of the present text (Chapters 3 and 4). At first one is led to restrict to those symmetric spaces, called here «special», for which the method applies in the same way as for Lie groups. But, when considering more general spaces, one needs to introduce a realvalued function $e(X, Y)$ of two tangent vectors $X, Y$ : identically 1 for special spaces, this function embodies the modification of the Kashiwara-Vergne method required for a general symmetric space. It can be constructed from its infinitesimal structure (the corresponding Lie triple system) and contains by itself much information about invariant analysis on the symmetric space, when transferred to its tangent space at the origin via the exponential mapping (hence the notation «e»): invariant differential operators, mean values, spherical functions are related to $e$. The search for such relations between analysis and infinitesimal properties is our main guiding line. 
Several recent works [1][12][6] have attracted attention on the non-unique ness in such constructions and encouraged me to rewrite my previous papers [43] - [47] entirely. Reorganizing them in a more synthetic way, we now separate the general use of $e$ in invariant analysis on a symmetric space (Chapter 3 ) from properties arising from a specific construction of this function (Chapter 4). Not only do we speak of «an $e$-function» instead of «the $e$-function», but we also add several unpublished results, some of them recently obtained. Many proofs have been rewritten. The Kashiwara-Vergne «conjecture» is henceforth a theorem and, using it for the group $G$, stronger results and easier proofs can be given for the main properties of $e$ on the symmetric space $G / H$ (Section 4.4).

Let us describe the contents in more detail.

Chapter 1, devoted to the Kashiwara-Vergne method for Lie groups, provides inspiration and motivation for its extension to symmetric spaces. It is however almost entirely independent of the sequel, its results being only used in Section 4.4.2. We give a complete proof of the conjecture for two important families of Lie groups (quadratic, resp. solvable, following [8], resp. [48]) and a brief overview of the latest proof by Alekseev and Torossian [7] for the general case.

Apart from Chapter 1 the whole paper focuses on a convolution transfer formula from a symmetric space $S=G / H$ to its tangent space $\mathfrak{s}$ at the origin. Working on suitable neighborhoods of the origin, we prove the existence of a (non-unique) function $e$ on $\mathfrak{s} \times \mathfrak{s}$ such that, for all $H$-invariant distributions $u, v$ on $\mathfrak{s}$ and all test functions $f$ on $\mathfrak{s}$,

$$
\left\langle\widetilde{u} *_{S} \widetilde{v}, \widetilde{f}\right\rangle=\langle u(X) \otimes v(Y), e(X, Y) f(X+Y)\rangle,
$$

where $X, Y$ denote variables in $\mathfrak{s}$. Let us explain the notation. The function $f$ on $\mathfrak{s}$ is transferred to $S$ as $\widetilde{f}$ by means of the exponential mapping Exp of the symmetric space and multiplication by some factor $j$, namely $f(X)=$ $j(X) \widetilde{f}(\operatorname{Exp} X)$. No specific choice of $j$ is necessary up to this point, though it soon becomes clear that the most interesting example is $j=J^{1 / 2}$, the square root of the Jacobian of Exp. This transfer extends to distributions by duality, giving two $H$-invariant distributions $\widetilde{u}, \widetilde{v}$ on $S$.

The convolution product $*_{S}$ is defined in Chapter 2, where a few examples are given.

In Chapter 3, regardless of any construction of $e$, we develop the outcome of the convolution formula in $H$-invariant analysis on $S$. If $e$ is identically 1 (the «special» case studied in Section 3.2), its right-hand side is simply $\left\langle u *_{\mathfrak{s}} v, f\right\rangle$, given by the classical abelian convolution on the vector space $\mathfrak{s}$. The $H$-invariant analysis on a special symmetric space thus boils down to classical Euclidean analysis on its tangent space at the origin. For general symmetric spaces the formula, applied to distributions supported at the origin, leads to an explicit description of $G$-invariant differential operators in 
the exponential chart and of the algebra $\mathbb{D}(S)$ of all these operators, which is commutative whenever $e(X, Y)=e(Y, X)$ (Section 3.3). Using $e$ we also give, if $H$ is compact, an expansion of mean value operators and spherical functions (Section 3.4). Invariant analysis is a well-known topic if $S$ is a Riemannian symmetric space of the noncompact type, and Section 3.6 is devoted to a discussion of the links between $e$ and the classical approach in this case. Our (partly conjectural) results of this section suggest the possibility of a far-reaching generalization of Duflo's isomorphism to symmetric spaces. In Section 3.7 we propose an explicit $e$-function for isotropic Riemannian symmetric spaces, arising from manipulations of integral formulas as explained in Section 3.5. Two technical proofs of this chapter are postponed to the Appendix.

In Chapter 4 we give a general construction of $e$ for arbitrary symmetric spaces, relying on the Campbell-Hausdorff formula in the spirit of the original Kashiwara-Vergne paper. This chapter contains our main results (Theorems $4.12,4.20,4.22,4.24)$, relating $e$ to the infinitesimal structure of the space. Let us call the reader's attention to an element $c(X, Y)$ of the group $H$, constructed in Section 4.2.5, which plays a key role at several places (e.g. Corollary 3.17, Proposition 3.21, Proposition 4.18, Theorem 4.24).

The theory extends to line bundles over a symmetric space. Though more general and arguably more natural (particularly for the bundle of halfdensities), this framework requires handling $H$-components and more cumbersome notation. We made the choice to deal with line bundles in specific sections of Chapters 2, 3 and 4 only. A character $\chi$ of the subgroup $H$ of $G$ defines a line bundle $L_{\chi}$ over $G / H$. The convolution transfer formula still holds for $H$-invariant sections of $L_{\chi}$ provided $e$ is replaced by $e_{\chi}$, the product of $e$ and a factor involving $\chi$. Again the formula leads to a description in terms of $e_{\chi}$ of the algebra $\mathbb{D}\left(L_{\chi}\right)$ of invariant differential operators and a new proof of Duflo's theorem on its commutativity for certain $\chi$, in particular for the bundle of half-densities (Sections 3.8 and 4.5).

Sections 3.9 and 4.7 list a few open questions.

Chapters 1 to 4 are, to a large extent, independent of each other. The Reader is assumed to have some familiarity with the basic theory of Lie groups and symmetric spaces, as can be gleaned from the books [27][28][37][58]. 


\section{Notation}

\subsection{General Notation}

The sign $:=$ indicates a definition.

$\mathbb{N}=\{0,1,2, \ldots\}$ denotes the set of natural integers, $\mathbb{Z}=\{\ldots,-2,-1,0,1,2, \ldots\}$ the ring of all integers, $\mathbb{R}$ the field of real numbers and $\mathbb{C}$ the field of complex numbers.

$\operatorname{sh} x=\left(e^{x}-e^{-x}\right) / 2, \operatorname{ch} x=\left(e^{x}+e^{-x}\right) / 2$, th $x=\operatorname{sh} x / \operatorname{ch} x$ and $\operatorname{coth} x=$ $\operatorname{ch} x / \operatorname{sh} x$ are the classical hyperbolic functions.

In Chapters 1 and 4 we shall use three commuting involutions acting on a function $f$ of two variables in a vector space

$$
f^{\vee}(X, Y):=f(-X,-Y), \bar{f}(X, Y):=f(Y, X), \widetilde{f}(X, Y):=f(-Y,-X) .
$$

Dots are used with several meanings, which should be clear from their context: if a group $G$ acts on a set, the action of $g \in G$ transforms a point $x$ of the set into $g \cdot x$.

$\langle.,$.$\rangle are duality brackets between a vector space and its dual, or sometimes$ denote a scalar product. ${ }^{t}$ means transpose.

$\operatorname{tr}_{V} u$ denotes the trace of an endomorphism $u$ of a finite dimensional vector space $V$ and $\operatorname{det}_{V} u$ its determinant. The subscripts $V$ may be dropped when no confusion arises.

Smooth means $C^{\infty}$ and supp denotes the support.

$\mathcal{D}(M)$ denotes the space of test functions on a manifold $M$, i.e. smooth complex-valued compactly supported functions on $M$, equipped with the Schwartz topology (see e.g. [28] p. 239). Its dual $\mathcal{D}^{\prime}(M)$ is the space of distributions on $M$.

If $f$ maps a manifold into another, $D_{x} f$ is the tangent map (differential) of $f$ at $x$. In case of several variables $x, y$ etc., $\partial_{x} f$ is the partial differential with respect to $x$.

Let us recall the classical multi-index notation when taking coordinates $\left(x_{1}, \ldots, x_{n}\right)$ in a vector space: for $\alpha=\left(\alpha_{1}, \ldots, \alpha_{n}\right) \in \mathbb{N}^{n}$

$$
\begin{aligned}
|\alpha| & =\alpha_{1}+\cdots+\alpha_{n}, \alpha !=\alpha_{1} ! \cdots \alpha_{n} ! \\
x^{\alpha} & =x_{1}^{\alpha_{1}} \cdots x_{n}^{\alpha_{n}}, \partial^{\alpha}=\partial_{1}^{\alpha_{1}} \cdots \partial_{n}^{\alpha_{n}}, \partial_{i}=\partial / \partial x_{i} .
\end{aligned}
$$




\subsection{Lie Groups and Lie Algebras}

The identity element of a Lie group $G$ is denoted by $e$ (not to be confused, of course, with a notation such as $e^{X}$ or $\left.e(X, Y)\right)$.

$L_{g}$, resp. $R_{g}$, is the left, resp. right, translation in $G$, that is $L_{g} x:=g x$ and $R_{g} x:=x g$ for $g, x \in G$.

For $X, Y$ in the Lie algebra $\mathfrak{g}$ of $G$, with bracket [.,.], we write ad $X(Y):=$ $[X, Y]$. The notation $x:=\operatorname{ad} X, y:=\operatorname{ad} Y$ will be frequently used in Chapters 1 and 4 ; for example $x y^{2} x Y=[X,[Y,[Y,[X, Y]]]]$ etc.

If $\mathfrak{a}, \mathfrak{b}$ are vector subspaces of $\mathfrak{g},[\mathfrak{a}, \mathfrak{b}]$ is the space of all finite sums $\sum_{i}\left[A_{i}, B_{i}\right]$ with $A_{i} \in \mathfrak{a}, B_{i} \in \mathfrak{b}$.

The adjoint representation Ad of $G$ on $\mathfrak{g}$ will most often be denoted by a dot: $g \cdot X:=\operatorname{Ad} g(X)$ for $g \in G, X \in \mathfrak{g}$.

The exponential mapping $\exp : \mathfrak{g} \rightarrow G$ will most often be written as $X \mapsto$ $\exp X=e^{X}$; thus $e^{g \cdot X}=g e^{X} g^{-1}$ and $\operatorname{Ad} e^{X}=e^{\text {ad } X}$.

The Campbell-Hausdorff formula expands $V(X, Y):=\log \left(e^{X} e^{Y}\right)$ as a series of brackets of $X$ and $Y$.

The differential at $X \in \mathfrak{g}$ of the exponential mapping and its Jacobian are

$$
D_{X} \exp =D_{e} L_{e^{X}} \circ \frac{1-e^{-x}}{x}, j(X)=\operatorname{det}_{\mathfrak{g}} \frac{1-e^{-x}}{x}
$$

with $x=\operatorname{ad} X$.

Caution: this notation $j$ is only used in Chapter 1 . In Chapters 3 and $4, j$ has a more general meaning (Definition 3.2).

\subsection{Symmetric Lie Algebras}

A symmetric Lie algebra is a couple $(\mathfrak{g}, \sigma)$ where $\mathfrak{g}$ is a (finite dimensional real) Lie algebra and $\sigma$ is an involutive automorphism of $\mathfrak{g}$. If $\mathfrak{h}$, resp. $\mathfrak{s}$, denotes the +1 , resp. -1 , eigenspace of $\sigma$ in $\mathfrak{g}$, we have the decomposition $\mathfrak{g}=\mathfrak{h} \oplus \mathfrak{s}$ with $[\mathfrak{h}, \mathfrak{h}] \subset \mathfrak{h},[\mathfrak{h}, \mathfrak{s}] \subset \mathfrak{s},[\mathfrak{s}, \mathfrak{s}] \subset \mathfrak{h}$ by the rule of signs.

The subspace $\mathfrak{h}_{*}:=[\mathfrak{s}, \mathfrak{s}]$ is an ideal of $\mathfrak{h}$.

The subspace $\mathfrak{s}$ is not in general a Lie algebra, but inherits from $\mathfrak{g}$ a structure of Lie triple system: denoting by $L(X, Y)$ the map $Z \mapsto[X, Y, Z]:=$ $[[X, Y], Z]$ from $\mathfrak{s}$ into itself, we have

(i) $L(X, X)=0$

(ii) $[X, Y, Z]+[Y, Z, X]+[Z, X, Y]=0$

(iii) $L(X, Y)$ is a derivation of the trilinear product $[., .,$.$] , that is$

$L(X, Y)[U, V, W]=[L(X, Y) U, V, W]+[U, L(X, Y) V, W]+[U, V, L(X, Y) W]$

for all $X, Y, Z, U, V, W \in \mathfrak{s}$ (see [37] p. 78). 


\subsection{Symmetric Spaces}

Throughout the text $G$ denotes a connected real Lie group and $H$ a closed subgroup of $G$. Let $S=G / H$ be the homogeneous space $S=G / H$ of left cosets $g H$, with $g \in G$. It will be convenient to assume $S$ is simply connected; this implies $H$ is connected.

Let $\widetilde{G}$ be the universal covering of $G$ with canonical projection $p: \widetilde{G} \rightarrow G$ and $\widetilde{H}=p^{-1}(H)$. From the simple connectedness of $G / H$ it follows that $\widetilde{H}$ is connected and $\widetilde{G} / \widetilde{H}=G / H$. Thus, whenever necessary, the group $G$ may be assumed to be simply connected too. These topological properties will only be useful to give a precise definition of the domains we are working on. The natural action of $G$ on $S$ is denoted by $\tau(g)\left(g^{\prime} H\right)=g \cdot g^{\prime} H:=g g^{\prime} H$ for $g, g^{\prime} \in G$, and $o=e H$ is taken as the origin of $S$.

A homogeneous space $S=G / H$ is a symmetric space if $G$ is equipped with an involutive automorphism $\sigma$ and $H$ lies between the fixed point subgroup of $\sigma$ in $G$ and its identity component (if connected, $H$ therefore equals this component). We still denote by $\sigma$ the corresponding automorphism of the Lie algebra $\mathfrak{g}$ of $G$, whence a symmetric Lie algebra $(\mathfrak{g}, \sigma)$. In the decomposition $\mathfrak{g}=\mathfrak{h} \oplus \mathfrak{s}, \mathfrak{h}$ is the Lie algebra of $H$ and $\mathfrak{s}$ identifies with the tangent space to $S$ at $o$. We denote by $H_{*}$ the connected Lie subgroup of $H$ with Lie algebra $\mathfrak{h}_{*}=[\mathfrak{s}, \mathfrak{s}]$.

The exponential mapping given by the canonical connection of the symmetric space is $\operatorname{Exp} X=e^{X} H, X \in \mathfrak{s}$, where $X \mapsto e^{X}$ is the exponential mapping of the group $G$. For $h \in H$ and $X \in \mathfrak{s}$ we have $h \cdot \operatorname{Exp} X=\operatorname{Exp}(h \cdot X)$.

For $X, Y$ in a neighborhood of the origin in $\mathfrak{s}$, the element $Z(X, Y)$ of $\mathfrak{s}$ defined by $\operatorname{Exp} Z(X, Y)=\exp X \cdot \operatorname{Exp} Y$ is the symmetric space analog of the Campbell-Hausdorff element $V(X, Y)$ of Lie groups.

The differential at $X \in \mathfrak{s}$ of the exponential mapping and its Jacobian are, with $x=\operatorname{ad} X$,

$$
D_{X} \operatorname{Exp}=D_{o} \tau\left(e^{X}\right) \circ \frac{\operatorname{sh} x}{x}, J(X)=\operatorname{det}_{\mathfrak{s}} \frac{\operatorname{sh} x}{x} .
$$

The symmetric space $S$ admits a $G$-invariant measure $d x$ if and only if the Lebesgue measure $d X$ of $\mathfrak{s}$ is $H$-invariant, that is $\left|\operatorname{det}_{\mathfrak{s}} \operatorname{Ad} h\right|=1$ for all $h \in H$. No absolute value is needed if $H$ is connected. The measures can then be normalized so that

$$
\int_{S} f(x) d x=\int_{\mathfrak{s}} f(\operatorname{Exp} X) J(X) d X
$$

if supp $f$ is contained in a suitable neighborhood of the origin.

The superscript $H$ on a space denotes the subspace of $H$-invariant elements. For example if $U$ is an $H$-invariant open subset of $S, \mathcal{D}(U)^{H}$ is the space of test functions $f$ such that supp $f \subset U$ and $f \circ \tau(h)=f$ for all $h \in H$. Similarly $\mathcal{D}^{\prime}(U)^{H}$ is the space of distributions $T$ on $U$ such that $\langle T, f\rangle=\langle T, f \circ \tau(h)\rangle$ for all $f \in \mathcal{D}(U), h \in H$. 
$\mathbb{D}(\mathfrak{s})=S(\mathfrak{s})$ is the algebra of linear differential operators with constant (complex) coefficients on $\mathfrak{s}$, canonically identified with the (complexified) symmetric algebra of $\mathfrak{s}$, and $\mathbb{D}(\mathfrak{s})^{H}=S(\mathfrak{s})^{H}$ is the subalgebra of $H$-invariant operators.

$\mathbb{D}(S)$ denotes the algebra of $G$-invariant linear differential operators on $S$. The specific notation for line bundles is explained in Section 2.2.

\subsection{Semisimple Notation}

In Proposition 3.7, Sections 3.6, 3.7 and the Appendix, we narrow our investigation to the case of a Riemannian symmetric space of the noncompact type. We shall then replace the above general notation by the classical semisimple notation as used in Helgason's books [27] - [29], to which we refer for details and proofs.

The symmetric space is then $S=G / K$, simply connected, where $G$ is a connected noncompact real semisimple Lie group with finite center and $K$ is a maximal compact subgroup; $K$ is connected. The involutive automorphism is the Cartan involution $\theta$ giving the decomposition $\mathfrak{g}=\mathfrak{k} \oplus \mathfrak{p}$. For $X, Y \in \mathfrak{g}$ let $\langle X, Y\rangle:=-B(X, \theta Y)$, where $B(X, Y):=\operatorname{tr}_{\mathfrak{g}}(\operatorname{ad} X \operatorname{ad} Y)$ is the Killing form. The Riemannian structure on $S$ is the $G$-invariant metric defined by the scalar product $\langle.,$.$\rangle restricted to \mathfrak{p}$. The exponential mapping Exp is then a global diffeomorphism of $\mathfrak{p}$ onto $S$.

Let $\mathfrak{a}$ be a maximal abelian subspace of $\mathfrak{p}$ and $\mathfrak{a}^{*}$ its dual space; the dimension of $\mathfrak{a}$ is the rank of $G / K$. A linear form $\alpha \in \mathfrak{a}^{*}$ is called a root of $(\mathfrak{g}, \mathfrak{a})$ if $\alpha \neq 0$ and $\mathfrak{g}_{\alpha} \neq\{0\}$, where

$$
\mathfrak{g}_{\alpha}:=\{X \in \mathfrak{g} \mid[H, X]=\alpha(H) X \text { for all } H \in \mathfrak{a}\} .
$$

The dimension $m_{\alpha}=\operatorname{dim} \mathfrak{g}_{\alpha}$ is the multiplicity of the $\operatorname{root} \alpha$. A point $H \in \mathfrak{a}$ is called regular if $\alpha(H) \neq 0$ for all roots $\alpha$. The set $\mathfrak{a}^{\prime}$ of regular elements has (finitely many) connected components, the Weyl chambers. Having picked one of them, called the positive Weyl chamber and denoted by $\mathfrak{a}^{+}$(with closure $\overline{\mathfrak{a}^{+}}$in $\mathfrak{g}$ ), we say a root is positive if it takes positive values on $\mathfrak{a}^{+}$. Let $\rho:=\frac{1}{2} \sum_{\alpha>0} m_{\alpha} \alpha \in \mathfrak{a}^{*}$ (sum over the set of positive roots $\alpha$ ). Let $M$, resp. $M^{\prime}$, be the centralizer, resp. normalizer, of $\mathfrak{a}$ in $K$. They are compact subgroups with the same Lie algebra. The quotient group $W:=M^{\prime} / M$ is a finite group called the Weyl group; it acts simply transitively on the set of Weyl chambers.

The choice of $\mathfrak{a}$ gives rise to the Iwasawa decomposition $\mathfrak{g}=\mathfrak{k} \oplus \mathfrak{a} \oplus \mathfrak{n}$ with $\mathfrak{n}=\oplus_{\alpha>0} \mathfrak{g}_{\alpha}$. The corresponding Lie subgroups of $G$ give the decomposition $G=K A N$, the map $(k, a, n) \mapsto g=$ kan being a diffeomorphism of $K \times A \times N$ onto $G$. 


\section{Chapter 1}

\section{The Kashiwara-Vergne Method for Lie Groups}

In this first chapter we motivate and explain the two equations (KV1) and (KV2) of the «Kashiwara-Vergne conjecture» for a Lie algebra and their application to analysis on the corresponding Lie group (transfer of convolution of invariant distributions, Duflo isomorphism); see Sections 1.2 to 1.5. Section 1.6, independent of the sequel, gives an elementary solution of (KV1) and $(\mathrm{KV} 2)$ for the algebra $\operatorname{sl}(2, \mathbb{R})$. After discussing two symmetries on the set of solutions (Section 1.7), we give a detailed proof of the conjecture in two important special cases: quadratic Lie algebras, solvable Lie algebras (Sections 1.8 and 1.9). The chapter ends with a brief survey of a proof in the general case due to Alekseev and Torossian (Section 1.10).

The main statements are Theorem 1.5 (the Kashiwara-Vergne equations (KV1) and (KV2) imply a convolution equality), Conjecture 1.6 (the Kashi wara-Vergne conjecture), Theorems 1.23 and 1.24 (the conjecture is true for quadratic or solvable Lie algebras), and Theorems 1.26 and 1.27 (the conjecture is true for all Lie algebras).

Chapter 1 provides motivation for a generalization to symmetric spaces in the sequel. However Chapter 3 and 4 are, to a large extent, independent from the present one.

\subsection{A General Convolution Problem}

Let $G$ be a (finite dimensional real) Lie group with Lie algebra $\mathfrak{g}$. The convolution of two distributions $u, v$ on the vector space $\mathfrak{g}$ is the distribution $u *_{\mathfrak{g}} v$ defined by

$$
\left\langle u *_{\mathfrak{g}} v, f\right\rangle:=\langle u(X) \otimes v(Y), f(X+Y)\rangle
$$


where $X, Y$ denote variables in $\mathfrak{g},\langle.,$.$\rangle the duality between distributions and$ functions and $f$ is an arbitrary test function (smooth and compactly supported) on $\mathfrak{g}$. Similarly the convolution of two distributions $U, V$ on $G$ is defined by

$$
\left\langle U *_{G} V, \varphi\right\rangle:=\langle U(g) \otimes V(h), \varphi(g h)\rangle
$$

where $g, h$ denote variables in $G$ and $\varphi$ is an arbitrary test function on $G$. These definitions make sense under suitable additional assumptions, e.g. if one of the distributions involved is compactly supported. They extend to distributions the addition of $\mathfrak{g}$, resp. the group law of $G$, as seen by applying them to Dirac measures. The product $*_{\mathfrak{g}}$ is commutative whereas in general $*_{G}$ is not.

The exponential mapping exp : $\mathfrak{g} \rightarrow G, X \mapsto \exp X=e^{X}$, is a diffeomorphism from a neigborhood of 0 in the Lie algebra onto a neighborhood of the identity in the group and our goal is to relate both convolutions by means of exp. From now on in this introduction we shall work on these neighborhoods without further mention. Let $f \mapsto \widetilde{f}$ denote the transfer of a function $f$ on $\mathfrak{g}$, defined by

$$
j(X)^{1 / 2} \widetilde{f}\left(e^{X}\right)=f(X)
$$

where $j(X):=\operatorname{det}\left(\left(1-e^{-\operatorname{ad} X}\right) / \operatorname{ad} X\right)$ is the Jacobian of exp. By duality the transfer $u \mapsto \widetilde{u}$ of a distribution $u$ on $\mathfrak{g}$ is defined by

$$
\langle\widetilde{u}, \widetilde{f}\rangle=\langle u, f\rangle
$$

for any test function $f$. Finally let us recall that a distribution $u$ on $\mathfrak{g}$ is $G$ invariant if $\langle u(X), f(g \cdot X)\rangle=\langle u(X), f(X)\rangle$ for any $f$ and any $g \in G$, where dot denotes the adjoint action of $G$ on its Lie algebra; $\widetilde{u}$ is then a central distribution on $G$, that is $\left\langle\widetilde{u}, \varphi_{g}\right\rangle=\langle\widetilde{u}, \varphi\rangle$ for any $\varphi$ and any $g \in G$, with $\varphi_{g}(h):=\varphi\left(g h g^{-1}\right)$.

Problem. Prove that

$$
\left(u *_{\mathfrak{g}} v\right)^{\sim}=\widetilde{u} *_{G} \widetilde{v}
$$

for any $G$-invariant distributions $u, v$ on $\mathfrak{g}$ (with suitable supports).

Note that $\langle\widetilde{v}(h), \varphi(g h)\rangle=\left\langle\widetilde{v}(h), \varphi\left(g^{-1}(g h) g\right)\right\rangle=\langle\widetilde{v}(h), \varphi(h g)\rangle$ if $\widetilde{v}$ is $G$ invariant, which implies the commutativity $\widetilde{u} *_{G} \widetilde{v}=\widetilde{v} *_{G} \widetilde{u}$. The problem would be impossible otherwise.

If $v$ is $G$-invariant and supported at the origin the convolution by $v$ is a $G$-invariant differential operator with constant coefficients on $\mathfrak{g}$ and the convolution by $\widetilde{v}$ is a bi-invariant differential operator on $G$. Equation (1.1) therefore reduces the study of the latter operators to the much easier case of the former.

Two previously known deep results motivate this problem:

- In 1965 Harish-Chandra [26] proved (1.1) for $G$ semisimple and $v$ supported at the origin. 
- In 1977 Duflo [18] proved (1.1) for all Lie groups $G$ and $u, v$ supported at the origin. One of his results can be stated as follows: let $P \in S(\mathfrak{g})^{G}$ be an invariant element of the symmetric algebra of $\mathfrak{g}$, identified with a $G$-invariant differential operator with constant coefficients $P\left(\partial_{X}\right)$ on $\mathfrak{g}$, and let $\gamma(P)$ be the differential operator on $G$ defined by

$$
\gamma(P) \varphi(g):=\left.P\left(\partial_{X}\right)\left(j(X)^{1 / 2} \varphi\left(g e^{X}\right)\right)\right|_{X=0}
$$

for any smooth function $\varphi$. Then $P \mapsto \gamma(P)$ is an isomorphism of algebras of $S(\mathfrak{g})^{G}$ onto $Z(\mathfrak{g})$, the center of the universal enveloping algebra of $\mathfrak{g}$, identified with the algebra of bi-invariant differential operators on $G$. This map is known as the Duflo isomorphism. It is related to by $(\gamma(P) \tilde{f})(e)=\left(P\left(\partial_{X}\right) f\right)(0)$, that is ${ }^{t} \gamma(P) \delta_{G}=\left({ }^{t} P\left(\partial_{X}\right) \delta_{\mathfrak{g}}\right)^{\sim}$ where ${ }^{t}$ denotes the transpose operator and $\delta$ the Dirac measure at the origin. Duflo's theorem is thus a consequence of (1.1), taking $P, Q \in S(\mathfrak{g})^{G}$ and $u={ }^{t} P \delta, v={ }^{t} Q \delta$. In the same paper Duflo also proved the local solvability of all (non-zero) bi-invariant differential operators on $G$. This follows again from (1.1), taking as $u$ a $G$-invariant fundamental solution of ${ }^{t} P\left(\partial_{X}\right)$ (see Raïs [40]): $\widetilde{u}$ is then a (local) fundamental solution of ${ }^{t} \gamma(P)$ whence its local solvability.

Harish-Chandra's and Duflo's proofs are both difficult and make use of the structure of the group. A direct proof of (1.1) would be more natural however, only relying on formal properties of the exponential mapping and its Jacobian. This has become possible thanks to the Kashiwara-Vergne method [30] which we now describe.

\subsection{The Kashiwara-Vergne Method}

Applied to a test function $\tilde{f}$ the left-hand side of (1.1) is

$$
\left\langle\left(u *_{\mathfrak{g}} v\right)^{\tilde{f}}, \widetilde{f}\right\rangle=\left\langle u *_{\mathfrak{g}} v, f\right\rangle=\langle u(X) \otimes v(Y), f(X+Y)\rangle
$$

and, according to our definitions, the right-hand side is

$$
\left\langle\widetilde{u} *_{G} \widetilde{v}, \widetilde{f}\right\rangle=\langle\widetilde{u}(g),\langle\widetilde{v}(h), \widetilde{f}(g h)\rangle\rangle=\langle\widetilde{u}(g), \widetilde{\varphi}(g)\rangle=\langle u, \varphi\rangle
$$

with $\widetilde{\varphi}(g)=\langle\widetilde{v}(h), \widetilde{f}(g h)\rangle$ i.e. $\varphi(X):=j(X)^{1 / 2}\left\langle\widetilde{v}(h), \widetilde{f}\left(e^{X} h\right)\right\rangle$. For fixed $X$ we may write $\widetilde{f}\left(e^{X} h\right)=\widetilde{\psi}(h)$ with $\psi(Y):=j(Y)^{1 / 2} \widetilde{f}\left(e^{X} e^{Y}\right)$, and here enters the Campbell-Hausdorff ${ }^{1}$ formula

$V(X, Y):=\log \left(e^{X} e^{Y}\right)=X+Y+\frac{1}{2}[X, Y]+\frac{1}{12}[X,[X, Y]]-\frac{1}{12}[Y,[X, Y]]+\cdots$

\footnotetext{
${ }^{1}$ According to [11] Campbell-Baker-Hausdorff-Dynkin (in chronological order of the contributions) should be a more appropriate terminology. On computational aspects of the formula, see e.g. the exposition in [13].
} 
expressing the group law in exponential coordinates (near the origin). Thus $\varphi(X)=j(X)^{1 / 2}\langle v, \psi\rangle$ with

$$
\psi(Y)=j(Y)^{1 / 2} \widetilde{f}\left(e^{V(X, Y)}\right)=\left(\frac{j(Y)}{j(V(X, Y))}\right)^{1 / 2} f(V(X, Y))
$$

and finally

$$
\left\langle\widetilde{u} *_{G} \widetilde{v}, \widetilde{f}\right\rangle=\left\langle u(X) \otimes v(Y),\left(\frac{j(X) j(Y)}{j(V(X, Y))}\right)^{1 / 2} f(V(X, Y))\right\rangle .
$$

Equation (1.1) is now equivalent to

$$
\langle u(X) \otimes v(Y), f(X+Y)\rangle=\left\langle u(X) \otimes v(Y),\left(\frac{j(X) j(Y)}{j(V(X, Y))}\right)^{1 / 2} f(V(X, Y))\right\rangle .
$$

One of the main ideas in [30] is to prove (1.2) by deformation. Let $\mathfrak{g}_{t}$ be the Lie algebra obtained by endowing the vector space $\mathfrak{g}$ with the bracket $[X, Y]_{t}=t[X, Y]$, where $t$ is a real parameter; thus $\mathfrak{g}_{0}$ is abelian and $\mathfrak{g}_{1}=\mathfrak{g}$. The corresponding function $V$ is

$V_{t}(X, Y)=t^{-1} V(t X, t Y)=X+Y+\frac{t}{2}[X, Y]+\frac{t^{2}}{12}[X,[X, Y]]-\frac{t^{2}}{12}[Y,[X, Y]]+\cdots$

for $t \neq 0$, and $V_{0}(X, Y)=X+Y$. It will therefore suffice to prove that, for any $G$-invariant distributions $u, v$ on $\mathfrak{g}$ and any test function $f$,

$$
\frac{\partial}{\partial t}\left\langle u(X) \otimes v(Y),\left(\frac{j(t X) j(t Y)}{j\left(t V_{t}(X, Y)\right)}\right)^{1 / 2} f\left(V_{t}(X, Y)\right)\right\rangle=0
$$

since both sides of (1.2) are the values of this bracket for $t=0$ and $t=1$ respectively.

This will follow from the Kashiwara-Vergne equations (KV1) and (KV2) which we now introduce and motivate.

\subsection{The Equation (KV1)}

When trying to make (1.3) explicit the first difficulty is to express $\partial_{t} V_{t}(X, Y)$. This will be achieved by a technique à la Moser. We shall need the partial differentials of $V_{t}$ at $(X, Y)$ defined by

$$
\left(\partial_{X} V_{t}\right) Z=\left.\partial_{s} V_{t}(X+s Z, Y)\right|_{s=0},\left(\partial_{Y} V_{t}\right) Z=\left.\partial_{s} V_{t}(X, Y+s Z)\right|_{s=0}
$$

for any $Z \in \mathfrak{g}$.

Let us look for a one parameter family of diffeomorphisms $\mathbf{F}_{t}$ of $\mathfrak{g} \times \mathfrak{g}$ into itself (in a neighborhood of the origin), starting from $\mathbf{F}_{0}=I d$, given by the 
adjoint action of $G \times G$ and transforming $V_{t}(X, Y)$ into its abelian analog $X+Y$, that is

$$
\begin{gathered}
\mathbf{F}_{t}(X, Y)=\left(X_{t}, Y_{t}\right):=\left(f_{t}(X, Y) \cdot X, g_{t}(X, Y) \cdot Y\right) \\
f_{0}(X, Y)=g_{0}(X, Y)=e \\
V_{t} \circ \mathbf{F}_{t}(X, Y)=V_{t}\left(X_{t}, Y_{t}\right)=X+Y
\end{gathered}
$$

with $f_{t}(X, Y), g_{t}(X, Y) \in G$. Since $V_{0}(X, Y)=X+Y,(1.5)$ is equivalent to

$$
\partial_{t} V_{t}+\left(\partial_{X} V_{t}\right) \partial_{t} X_{t}+\left(\partial_{Y} V_{t}\right) \partial_{t} Y_{t}=0
$$

where all derivatives of $V_{t}$ are computed at $\left(X_{t}, Y_{t}\right)$. Let $\left(F_{t}, G_{t}\right): \mathfrak{g} \times \mathfrak{g} \rightarrow$ $\mathfrak{g} \times \mathfrak{g}$ be the time-dependent vector field (near the origin) which generates $\left(f_{t}, g_{t}\right)$ :

$$
\begin{aligned}
& \partial_{t} f_{t}(X, Y)=\left(D_{e} R_{f_{t}(X, Y)}\right) F_{t}\left(X_{t}, Y_{t}\right) \\
& \partial_{t} g_{t}(X, Y)=\left(D_{e} R_{g_{t}(X, Y)}\right) G_{t}\left(X_{t}, Y_{t}\right)
\end{aligned}
$$

where $R_{a}$ denotes the right translation $g \mapsto g a$ in $G$ and $D_{e} R_{a}$ its tangent map at the identity. Then (1.4) implies

$$
\partial_{t} X_{t}=\left[F_{t}\left(X_{t}, Y_{t}\right), X_{t}\right], \partial_{t} Y_{t}=\left[G_{t}\left(X_{t}, Y_{t}\right), Y_{t}\right] .
$$

Indeed (1.8) is easily checked in a matrix Lie group, where $f_{t} \cdot X=f_{t} X f_{t}^{-1}$. For general Lie groups, one notes that the adjoint representation satisfies $\left(\operatorname{Ad} \circ R_{g}\right)\left(e^{t X}\right)=\operatorname{Ad}\left(e^{t X}\right) \circ \operatorname{Ad} g$ for $g \in G$ and $X \in \mathfrak{g}$, whence by differentiation $\left(D_{g} \operatorname{Ad} \circ D_{e} R_{g}\right) X=\operatorname{ad} X \circ \operatorname{Ad} g$ and the result. From (1.6) and (1.8) we see that (1.5) is equivalent to

$$
\partial_{t} V_{t}=\left(\partial_{X} V_{t}\right)\left[X, F_{t}\right]+\left(\partial_{Y} V_{t}\right)\left[Y, G_{t}\right]
$$

where all functions are now evaluated at $(X, Y)$.

With applications to analysis on $G$ in mind, let us introduce the neighborhoods we shall be working with. Let $\mathfrak{g}^{\prime}$ be an open neighborhood of the origin in $\mathfrak{g}$, invariant under all automorphisms of the Lie algebra $\mathfrak{g}$ and all scalings $X \mapsto t X$ for $t \in[-1,1]$, and such that exp : $\mathfrak{g}^{\prime} \rightarrow \exp \mathfrak{g}^{\prime}$ is a diffeomorphism. If $G$ is connected and simply connected one may take as $\mathfrak{g}^{\prime}$ the set of all $X \in \mathfrak{g}$ such that $|\operatorname{Im} \lambda|<\pi$ for all eigenvalues $\lambda$ of ad $X$ (see [58] p. 113). If $G$ is a solvable exponential group one may take $\mathfrak{g}^{\prime}=\mathfrak{g}$, $\exp \mathfrak{g}^{\prime}=G$. Having chosen such a $\mathfrak{g}^{\prime}$ let

$$
\mathcal{U}:=\left\{(X, Y) \mid X, Y \in \mathfrak{g}^{\prime} \text { and } e^{t X} e^{t Y} \in \exp \mathfrak{g}^{\prime} \text { for all } t \in[0,1]\right\} .
$$

The main properties of $\mathcal{U}$ are summarized in the following easy lemma.

Lemma 1.1 This set $\mathcal{U}$ is a connected open neighborhood of $(0,0)$ in $\mathfrak{g} \times \mathfrak{g}$, invariant under all maps $(X, Y) \mapsto(t X, t Y)$ for $t \in[-1,1],(X, Y) \mapsto(Y, X)$ and $(X, Y) \mapsto(g \cdot X, g \cdot Y)$ for $g \in G$. The map $(t, X, Y) \mapsto V(t X, t Y)=$ $\log \left(e^{t X} e^{t Y}\right)$ is analytic from an open subset of $\mathbb{R} \times \mathfrak{g} \times \mathfrak{g}$ containing $[-1,1] \times \mathcal{U}$ into $\mathfrak{g}^{\prime}$. 
Since $V(0,0)=0$ the map $(t, X, Y) \mapsto V_{t}(X, Y)=t^{-1} V(t X, t Y)$ is analytic too in the same open set.

Proof. Let $\left(X_{0}, Y_{0}\right) \in \mathcal{U}$ and let $C$ be a compact neighborhood of $\left(X_{0}, Y_{0}\right)$ in $\mathfrak{g}^{\prime} \times \mathfrak{g}^{\prime}$. The map $(t, X, Y) \mapsto f(t, X, Y):=e^{t X} e^{t Y}$ is uniformly continuous on $[0,1] \times C$ (with respect to some distance on $G$ ) therefore, for $C$ small enough, $f(t, X, Y)$ belongs to the open set $\exp \mathfrak{g}^{\prime}$ for all $t \in[0,1]$ and $(X, Y) \in C$. Thus $C \subset \mathcal{U}$ and $\mathcal{U}$ is open.

$\mathcal{U}$ is star-shaped with respect to $(0,0)$ hence connected.

The invariance of $\mathcal{U}$ under $(X, Y) \mapsto(g \cdot X, g \cdot Y)$ is clear. Taking $g=$ $e^{t Y}$ we have $g f(t, X, Y) g^{-1}=f(t, Y, X)$ and we infer the invariance under $(X, Y) \mapsto(Y, X)$ then, from $f(t,-X,-Y)=f(t, Y, X)^{-1}$, the invariance under $(X, Y) \mapsto(-X,-Y)$.

The map $(t, X, Y) \mapsto V(t X, t Y)=\log f(t, X, Y) \in \mathfrak{g}^{\prime}$ is analytic in the open set $f^{-1}\left(\exp \mathfrak{g}^{\prime}\right)$, which contains $[-1,1] \times \mathcal{U}$.

The notation $x:=\operatorname{ad} X, y:=\operatorname{ad} Y$ will be used throughout. The partial differentials of $V(X, Y)$ are given by the next lemma.

Lemma 1.2 For $(X, Y) \in \mathcal{U}$ we have, with $v=\operatorname{ad} V(X, Y)$,

$$
\frac{1-e^{-v}}{v} \circ \partial_{X} V=e^{-y} \frac{1-e^{-x}}{x}, \frac{1-e^{-v}}{v} \circ \partial_{Y} V=\frac{1-e^{-y}}{y} .
$$

Proof. As above let $L_{g}$, resp. $R_{g}$, denote the left, resp. right, translation by $g$ in $G$ and $D_{e} L_{g}, D_{e} R_{g}$ their differentials at the origin. Remembering the equality $e^{V}=e^{X} e^{Y}$ and the differential (1) of exp, differentiation with respect to $X$ gives

$$
D_{e} L_{e^{V}} \circ \frac{1-e^{-v}}{v} \circ \partial_{X} V=D_{e^{X}} R_{e^{Y}} \circ D_{e} L_{e^{X}} \circ \frac{1-e^{-x}}{x} .
$$

But $D_{e^{X}} R_{e^{Y}} \circ D_{e} L_{e^{X}}$ is the differential at $e$ of the map $g \mapsto e^{X} g e^{Y}=$ $e^{V}\left(e^{-Y} g e^{Y}\right)$, hence

$$
D_{e^{X}} R_{e^{Y}} \circ D_{e} L_{e^{X}}=D_{e} L_{e^{V}} \circ \operatorname{Ad}\left(e^{-Y}\right)=D_{e} L_{e^{V}} \circ e^{-y}
$$

and the first formula. The proof for $\partial_{Y} V$, similar and easier, is left to the Reader.

Proposition 1.3 Let $^{2} F, G$ be given elements of $\mathfrak{g}$ and let $(X, Y) \in \mathcal{U}$. The following are equivalent:

(i) $\left.\partial_{t} V_{t}(X, Y)\right|_{t=1}=\left(\partial_{X} V\right)(X, Y)[X, F]+\left(\partial_{Y} V\right)(X, Y)[Y, G]$

(ii) $V(Y, X)=X+Y-\left(1-e^{-x}\right) F-\left(e^{y}-1\right) G$.

\footnotetext{
${ }^{2}$ No confusion should arise between this $G$ and the group $G$ !
} 
Proof. For $(X, Y) \in \mathcal{U}, V(X, Y)$ belongs to $\mathfrak{g}^{\prime}$ so that, exp being a diffeomorphism on $\mathfrak{g}^{\prime}$, the map $\left(1-e^{-v}\right) / v$ is an invertible endomorphism of $\mathfrak{g}$. The definition of $V_{t}$ gives $\left.\partial_{t} V_{t}(X, Y)\right|_{t=1}=\left(\partial_{X} V\right) X+\left(\partial_{Y} V\right) Y-V$; (i) is thus equivalent to $\left(\partial_{X} V\right) X+\left(\partial_{Y} V\right) Y-V=\left(\partial_{X} V\right) x F+\left(\partial_{Y} V\right) y G$ or, applying $\left(1-e^{-v}\right) / v$ to both sides,

$$
e^{-y} X+Y-V(X, Y)=e^{-y}\left(1-e^{-x}\right) F+\left(1-e^{-y}\right) G
$$

by Lemma 1.2. But $e^{V(X, Y)}=e^{X} e^{Y}=e^{-Y}\left(e^{Y} e^{X}\right) e^{Y}=e^{-Y} e^{V(Y, X)} e^{Y}$, therefore $V(X, Y)=e^{-y} V(Y, X)$ and the above equation is equivalent to (ii).

Summarizing, assume we have two $\mathfrak{g}$-valued maps $F, G$ analytic in a neighborhood of the origin in $\mathfrak{g} \times \mathfrak{g}($ contained in $\mathcal{U})$ such that $F(0,0)=G(0,0)=0$ and the first Kashiwara-Vergne equation

$$
V(Y, X)=\log \left(e^{Y} e^{X}\right)=X+Y-\left(1-e^{-x}\right) F(X, Y)-\left(e^{y}-1\right) G(X, Y)
$$

holds on this neighborhood. Then, replacing $(X, Y)$ by $(t X, t Y)$ and setting $F_{t}(X, Y)=t^{-1} F(t X, t Y), G_{t}(X, Y)=t^{-1} G(t X, t Y)$, Proposition 1.3 shows that (1.9) holds true. Hence, defining $f_{t}, g_{t}$ by the differential equations (1.7):

$$
\begin{aligned}
& \partial_{t} f_{t}(X, Y)=\left(D_{e} R_{f_{t}(X, Y)}\right) F_{t}\left(f_{t}(X, Y) \cdot X, g_{t}(X, Y) \cdot Y\right), f_{0}=e \\
& \partial_{t} g_{t}(X, Y)=\left(D_{e} R_{g_{t}(X, Y)}\right) G_{t}\left(f_{t}(X, Y) \cdot X, g_{t}(X, Y) \cdot Y\right), g_{0}=e
\end{aligned}
$$

and $X_{t}, Y_{t}$ by $X_{t}=f_{t} \cdot X, Y_{t}=g_{t} \cdot Y$ we have (1.8):

$$
\begin{aligned}
\partial_{t} X_{t} & =\left[F_{t}\left(X_{t}, Y_{t}\right), X_{t}\right], X_{0}=X \\
\partial_{t} Y_{t} & =\left[G_{t}\left(X_{t}, Y_{t}\right), Y_{t}\right], Y_{0}=Y
\end{aligned}
$$

and it follows that (1.5) is satisfied (near the origin):

$$
V_{t} \circ \mathbf{F}_{t}(X, Y)=X+Y \text { with } \mathbf{F}_{t}(X, Y)=\left(f_{t}(X, Y) \cdot X, g_{t}(X, Y) \cdot Y\right) .
$$

Besides $f_{t}(X, Y)=f(t X, t Y), g_{t}(X, Y)=g(t X, t Y)$ with $f:=f_{1}, g:=g_{1}$, by uniqueness of solutions of (1.7).

\subsection{The Equation (KV2)}

Let us go back to (1.3). Assuming (KV1) holds, for any function $f$ on $\mathfrak{g}$ the derivative of $f\left(V_{t}(X, Y)\right)$ is, by $(1.9)$,

$$
\partial_{t} f\left(V_{t}(X, Y)\right)=D_{F_{t}, G_{t}}\left(f \circ V_{t}\right)(X, Y)
$$

where we have set, for any $A, B \in \mathfrak{g}$ and any function $\varphi$ on $\mathfrak{g} \times \mathfrak{g}$,

$D_{A, B} \varphi(X, Y):=\left(\partial_{X} \varphi\right)[X, A]+\left(\partial_{Y} \varphi\right)[Y, B]=\left.\partial_{s} \varphi\left(e^{-s A} \cdot X, e^{-s B} \cdot Y\right)\right|_{s=0}$. 
The $G$-invariance of $j$ implies $D_{A, B}\left(j(X)^{1 / 2} j(Y)^{1 / 2}\right)=0$ and we obtain, for fixed $t_{0}$,

$$
\begin{aligned}
\left.\partial_{t}\left(\left(\frac{j\left(t_{0} X\right) j\left(t_{0} Y\right)}{j\left(t_{0} V_{t}(X, Y)\right)}\right)^{1 / 2} f\left(V_{t}(X, Y)\right)\right)\right|_{t=t_{0}} & = \\
& =D_{F_{t_{0}}, G_{t_{0}}}\left(\left(\frac{j\left(t_{0} X\right) j\left(t_{0} Y\right)}{j\left(t_{0} V_{t_{0}}(X, Y)\right)}\right)^{1 / 2} f\left(V_{t_{0}}(X, Y)\right)\right)
\end{aligned}
$$

Moreover $j(X)=\operatorname{det}\left(\left(1-e^{-x}\right) / x\right)$ implies

$$
\partial_{t} \log j(t X)=\operatorname{tr}_{\mathfrak{g}}\left(\frac{x}{e^{t x}-1}-\frac{1}{t}\right)
$$

(where $\operatorname{tr}_{\mathfrak{g}}$ denotes the trace of an endomorphism of $\mathfrak{g}$ ), therefore

$$
\left.\partial_{t}\left(\frac{j(t X) j(t Y)}{j\left(t V_{t_{0}}(X, Y)\right)}\right)^{1 / 2}\right|_{t=t_{0}}=T_{t_{0}}(X, Y)\left(\frac{j\left(t_{0} X\right) j\left(t_{0} Y\right)}{j\left(t_{0} V_{t_{0}}(X, Y)\right)}\right)^{1 / 2}
$$

with $x=\operatorname{ad} X, y=\operatorname{ad} Y, v_{t}=\operatorname{ad} V_{t}(X, Y)$ and

$$
T_{t}(X, Y):=\frac{1}{2} \operatorname{tr}_{\mathfrak{g}}\left(\frac{x}{e^{t x}-1}+\frac{y}{e^{t y}-1}-\frac{v_{t}}{e^{t v_{t}}-1}-\frac{1}{t}\right) .
$$

Gathering both pieces we infer that the left-hand side of (1.3) is

$$
\begin{aligned}
& \partial_{t}\left\langle u(X) \otimes v(Y),\left(\frac{j(t X) j(t Y)}{j\left(t V_{t}(X, Y)\right)}\right)^{1 / 2} f\left(V_{t}(X, Y)\right)\right\rangle= \\
= & \left\langle u(X) \otimes v(Y),\left(D_{F_{t}, G_{t}}+T_{t}(X, Y)\right)\left(\frac{j(t X) j(t Y)}{j\left(t V_{t}(X, Y)\right)}\right)^{1 / 2} f\left(V_{t}(X, Y)\right)\right\rangle .
\end{aligned}
$$

Up to now the invariance of the distributions $u, v$ has never been used.

Lemma 1.4 Let $u$ be a $G$-invariant distribution on an open subset $U$ of $\mathfrak{g}$. Then, for any smooth map $F: U \rightarrow \mathfrak{g}$ such that $\operatorname{supp} u \cap \operatorname{supp} F$ is compact,

$$
\left\langle u(X), \operatorname{tr}_{\mathfrak{g}}\left(x \circ \partial_{X} F(X)\right)\right\rangle=0 .
$$

Proof. Let $F(X)=\sum_{i} F_{i}(X) E_{i}$ be the decomposition of $F(X)$ according to a fixed basis $\left(E_{i}\right)$ of $\mathfrak{g}$. The invariance of $u$ implies

$$
\left.\partial_{s}\left\langle u(X), F_{i}\left(e^{-s \operatorname{ad} E_{i}} X\right)\right\rangle\right|_{s=0}=\left\langle u(X), \partial_{X} F_{i}(X)\left[X, E_{i}\right]\right\rangle=0 .
$$

In the space $\mathfrak{g}^{*} \otimes \mathfrak{g}$ of endomorphisms of $\mathfrak{g}$ we have

$$
x \circ \partial_{X} F(X)=\sum_{i} \partial_{X} F_{i}(X) \otimes\left[X, E_{i}\right]
$$


hence $\operatorname{tr}\left(x \circ \partial_{X} F(X)\right)=\sum_{i} \partial_{X} F_{i}(X)\left[X, E_{i}\right]$ and the lemma.

Applied to $F(X)=\varphi(X) A(X)$ where $\varphi$ is a scalar function and $A$ is $\mathfrak{g}-$ valued, the lemma gives $\left\langle u,\left(\partial_{X} \varphi\right)[X, A]\right\rangle=-\left\langle u, \operatorname{tr}\left(x \circ \partial_{X} A\right) \varphi\right\rangle$. Replacing $\mathfrak{g}$ by $\mathfrak{g} \times \mathfrak{g}, u$ by $u \otimes v$ etc. we obtain

$$
\begin{aligned}
& \left\langle u(X) \otimes v(Y), D_{F_{t}, G_{t}} \varphi(X, Y)\right\rangle= \\
& =-\left\langle u(X) \otimes v(Y), \operatorname{tr}\left(x \circ \partial_{X} F_{t}+y \circ \partial_{Y} G_{t}\right) \varphi(X, Y)\right\rangle .
\end{aligned}
$$

We conclude that (1.3), therefore the convolution identity (1.1), will hold if $F$ and $G$ satisfy (KV1) and the second Kashiwara-Vergne equation with $x=\operatorname{ad} X, y=\operatorname{ad} Y, v=\operatorname{ad} V(X, Y)$

$$
\operatorname{tr}_{\mathfrak{g}}\left(x \circ \partial_{X} F+y \circ \partial_{Y} G\right)=\frac{1}{2} \operatorname{tr}_{\mathfrak{g}}\left(\frac{x}{e^{x}-1}+\frac{y}{e^{y}-1}-\frac{v}{e^{v}-1}-1\right) .
$$

An equivalent form of (KV2) is

$$
\operatorname{tr}_{\mathfrak{g}}\left(x \circ \partial_{X} F+y \circ \partial_{Y} G\right)=\frac{1}{2} \operatorname{tr}_{\mathfrak{g}}\left(\frac{x}{2} \operatorname{coth} \frac{x}{2}+\frac{y}{2} \operatorname{coth} \frac{y}{2}-\frac{v}{2} \operatorname{coth} \frac{v}{2}-1\right) .
$$

Indeed $x /\left(e^{x}-1\right)=\frac{x}{2} \operatorname{coth} \frac{x}{2}-\frac{x}{2}$ (which expands by means of the Bernoulli numbers); besides, the Campbell-Hausdorff formula shows $V-X-Y$ is a sum of brackets hence, by the adjoint representation, $v-x-y$ is a sum of brackets and $\operatorname{tr}(v-x-y)=0$. The even function $\frac{x}{2} \operatorname{coth} \frac{x}{2}$ will be more convenient for us in the sequel.

Summarizing, we obtain the following theorem, which is the raison d'être of the conjecture in the next section.

Theorem 1.5 Let ${ }^{3} G$ be a real Lie group with Lie algebra $\mathfrak{g}$, and let $F, G$ be two $\mathfrak{g}$-valued functions, analytic in a neighborhood of the origin in $\mathfrak{g} \times \mathfrak{g}$ such that $F(0,0)=G(0,0)=0$ and (KV1) and (KV2) hold. Then the convolution equality

$$
\left(u *_{\mathfrak{g}} v\right)^{\sim}=\widetilde{u} *_{G} \widetilde{v}
$$

holds on a neighborhood of the origin in $G$, for any $G$-invariant distributions $u, v$ on $\mathfrak{g}$ with suitable supports. In particular, if $F, G$ are analytic in $\mathcal{U}$ and $\operatorname{supp} v=\{0\}$, the equality $\left\langle\left(u *_{\mathfrak{g}} v\right)^{\sim}, \widetilde{f}\right\rangle=\left\langle\widetilde{u} *_{G} \widetilde{v}, \widetilde{f}\right\rangle$ holds for any $G-$ invariant distribution $u$ on an open subset of $\mathfrak{g}^{\prime}$ and any $f \in C^{\infty}(\mathfrak{g})$ such that $\operatorname{supp} u \cap \operatorname{supp} f$ is a compact subset of $\mathfrak{g}^{\prime}$.

The open sets $\mathfrak{g}^{\prime}$ and $\mathcal{U}$ have been introduced before Lemma 1.1. As already noted in Section 1.1 the case $\operatorname{supp} v=\{0\}$ leads to the main applications of the theorem: local solvability of bi-invariant differential operators and Duflo's isomorphism.

\footnotetext{
${ }^{3}$ With apologies for this double meaning of $G$, not too bothersome hopefully!
} 
Proof. The discussion in Sections 1.2 - 1.4 shows that (1.3) holds true, hence $\left\langle\left(u *_{\mathfrak{g}} v\right)^{\sim}, \widetilde{f}\right\rangle=\left\langle\widetilde{u} *_{G} \widetilde{v}, \widetilde{f}\right\rangle$.

As regards supports, the calculations are valid if, for all $t \in[0,1]$, ( $\operatorname{supp} u \times \operatorname{supp} v) \cap \operatorname{supp}\left(f \circ V_{t}\right)$ is a compact subset of the neighborhood where $F$ and $G$ are defined (supposed included in the set $\mathcal{U}$ of (1.10)). If this neighborhood is $\mathcal{U}$ itself and $\operatorname{supp} v=\{0\}$ we obtain the last result of the theorem, since $V_{t}(X, 0)=X$ and $\mathcal{U} \cap(\mathfrak{g} \times\{0\})=\mathfrak{g}^{\prime}$.

\subsection{The Kashiwara-Vergne Conjecture}

We shall now give a precise statement of the Kashiwara-Vergne «conjecture» - henceforth a theorem, now completely proved by two different methods in [5] and [7].

\subsubsection{The Conjecture}

Let $\mathbb{K}$ be a commutative field of characteristic zero and $\mathfrak{l}_{2}$ the free Lie algebra with two generators $X, Y$ over $\mathbb{K}$, completed with respect to its natural gradation (the generators having degree 1 ). The elements of $\mathfrak{l}_{2}$ are the formal series $F(X, Y)$ (without constant term) of $X, Y$ and iterated brackets of $X$ and $Y$, with coefficients in $\mathbb{K}$. We call them Lie series in $X, Y$ for short; we only consider series without zero order term. Two fundamental examples of Lie series are the Campbell-Hausdorff series

$$
\begin{aligned}
& V=V(X, Y):=\log \left(e^{X} e^{Y}\right)=X+Y+\frac{1}{2}[X, Y]+\cdots \in \mathfrak{l}_{2} \\
& \bar{V}=V(Y, X):=\log \left(e^{Y} e^{X}\right)=X+Y-\frac{1}{2}[X, Y]+\cdots \in \mathfrak{l}_{2} .
\end{aligned}
$$

If $X, Y$ are elements of a finite dimensional Lie algebra $\mathfrak{g}$ over $\mathbb{K}=\mathbb{R}$, the inclusion $\{X, Y\} \hookrightarrow \mathfrak{g}$ extends, by the universal property of free Lie algebras, to a morphism of Lie algebras from absolutely convergent formal series in some neighborhood of the origin (with respect to a norm on $\mathfrak{g}$ ) into $\mathfrak{g}$. In $\mathfrak{l}_{2}$ as well as $\mathfrak{g}$ we denote by $x=\operatorname{ad} X$ the map $Z \mapsto[X, Z]$ and similarly $y=\operatorname{ad} Y, v=\operatorname{ad} V$.

Conjecture 1.6 There exist two Lie series $F, G \in \mathfrak{l}_{2}$ such that

$$
V(Y, X)=X+Y-\left(1-e^{-x}\right) F(X, Y)-\left(e^{y}-1\right) G(X, Y)
$$

and, for every finite dimensional real Lie algebra $\mathfrak{g}$, these series define analytic functions of $(X, Y)$ in a neighborhhood of the origin in $\mathfrak{g} \times \mathfrak{g}$ (contained in the set $\mathcal{U}$ defined by (1.10)), satisfying the trace condition

$$
\operatorname{tr}_{\mathfrak{g}}\left(x \circ \partial_{X} F+y \circ \partial_{Y} G\right)=\frac{1}{2} \operatorname{tr}_{\mathfrak{g}}\left(\frac{x}{2} \operatorname{coth} \frac{x}{2}+\frac{y}{2} \operatorname{coth} \frac{y}{2}-\frac{v}{2} \operatorname{coth} \frac{v}{2}-1\right) .
$$


Here, for any $Z \in \mathfrak{g}, \partial_{X} F(X, Y) Z=\left.\partial_{t} F(X+t Z, Y)\right|_{t=0}$ and $\partial_{Y} G(X, Y) Z=$ $\left.\partial_{t} G(X, Y+t Z)\right|_{t=0}$.

The original statement of the conjecture in [30] was «For any finitedimensional Lie algebra there exist $F, G$, analytic near $(0,0)$, such that (KV1) and (KV2)». The hybrid statement chosen here, with (KV1) expressed within the framework of free Lie algebras, ensures that $F$ and $G$ are universal solutions of the problem, regardless of any specific relation between $X$ and $Y$ which may hold in such-and-such Lie algebra $\mathfrak{g}$. Of course the trace equation cannot be written as such for free algebras. However Corollary 1.22 below shows that (KV1) implies some formal analogue of (KV2).

Various equivalent forms of the conjecture are given in [5] (see also [57]).

\subsubsection{Remarks on the Conjecture}

a. Solving (KV1) alone is easy: the Campbell-Hausdorff series may be written as $\bar{V}=X+Y+[X, A]+[Y, B]$ with $A, B \in \mathfrak{l}_{2}$, hence (KV1) with $F, G \in \mathfrak{l}_{2}$ defined by

$$
F=\frac{x}{e^{-x}-1} A, G=\frac{y}{1-e^{y}} B .
$$

We refer to Burgunder [12] (Theorems 42 and 56) for a detailed formal study and explicit resolution of (KV1) by means of the Euler and Dynkin idempotents.

For $\mathbb{K}=\mathbb{R}$ closed formulas for $A, B, F, G$ can be obtained as follows. Let $V(t):=\log \left(e^{t X} e^{t Y}\right)$ and $v(t):=\operatorname{ad} V(t)=\log \left(e^{t x} e^{t y}\right)$. By Lemma 1.2 the derivative $V^{\prime}(t)$ with respect to $t$ is given by $\left(\left(1-e^{-v(t)}\right) / v(t)\right) V^{\prime}(t)=$ $e^{-t y}(X+Y)$ hence

$$
V=\int_{0}^{1} \frac{v(t)}{1-e^{-v(t)}} e^{-t y} d t(X+Y), \bar{V}=\int_{0}^{1} e^{t y} \frac{v(t)}{e^{v(t)}-1} d t(X+Y) .
$$

The latter formula follows from the former by exchanging $X, Y$ and observing that (in obvious notation) $\bar{v}(t)=e^{t y} v(t) e^{-t y}$. Let

$$
\psi(v):=\partial_{v}\left(\frac{v}{2} \operatorname{coth} \frac{v}{2}\right)=\frac{1}{2} \frac{\operatorname{sh} v-v}{\operatorname{ch} v-1} .
$$

Elementary computations show that the above expression of $\bar{V}$ may be rewritten as

$$
V(Y, X)=X+Y-\left(1-e^{-x}\right) F(X, Y)-\left(e^{y}-1\right) G(X, Y)
$$

with

$$
\begin{aligned}
& F(X, Y)=\int_{0}^{1} \frac{1-e^{-t x}}{1-e^{-x}}\left(\psi(v(t))+\frac{1}{2}\right) d t(X+Y) \\
& G(X, Y)=\int_{0}^{1} \frac{e^{t y}-1}{e^{y}-1}\left(\psi(v(t))-\frac{1}{2}\right) d t(X+Y)=F(-Y,-X) .
\end{aligned}
$$


These $F, G$ belong to $\mathfrak{l}_{2}$, satisfy (KV1) and define analytic functions of $(X, Y)$ in the open set $\mathcal{U}(1.10)$ : the analyticity of $x /\left(1-e^{-x}\right), v(t), v(t) \operatorname{coth} v(t) / 2$ and $\psi(v(t))$ follows from the definitions of $\mathfrak{g}^{\prime}$ and $\mathcal{U}$.

In order to prove that $F, G$ are given by convergent series of Lie brackets of $X, Y$ in a neighborhhod of $(0,0)$, we use a slightly different expression. Let

$$
\omega(u):=(1-u) \frac{u+\log (1-u)}{u^{2}}=-\frac{1}{2}+\sum_{n=1}^{\infty} \frac{u^{n}}{(n+1)(n+2)},
$$

so that $\psi(v)+\frac{1}{2}=-\omega(u)$ if $u=1-e^{v}$ and let

$$
A(X, Y):=\int_{0}^{1} \frac{1-e^{-t x}}{x} \omega(u(t)) d t(X+Y), B(X, Y):=A(-Y,-X)
$$

with $u(t):=1-e^{t x} e^{t y}$. Let $\|$.$\| be a norm on \mathfrak{g}$ such that ${ }^{4}\|[X, Y]\| \leq\|X\|\|Y\|$ for all $X, Y \in \mathfrak{g}$; the corresponding operator norm satisfies $\|x\|=\|$ ad $X \| \leq$ $\|X\|$. We claim that

$$
V(Y, X)=X+Y+[X, A(X, Y)]+[Y, B(X, Y)],
$$

the expressions $A, B, F=\left(x /\left(e^{-x}-1\right)\right) A$ and $G=\left(y /\left(e^{y}-1\right)\right) B$ are given by convergent series of Lie brackets for $\|X\|+\|Y\|<\log 2=0.693 \ldots$ and $F, G$ coincide with the above solutions (1.11) of (KV1).

Indeed the assumption on $X, Y$ implies $\|u(t)\| \leq e^{t(\|x\|+\|y\|)}-1<1$ for $0 \leq t \leq 1$, therefore

$$
A(X, Y)=\int_{0}^{1} \sum_{m \geq 0} \frac{(-t)^{m+1}}{(m+1) !} x^{m}\left(-\frac{1}{2}+\sum_{n \geq 1} \frac{u(t)^{n}}{(n+1)(n+2)}\right) d t(X+Y)
$$

where, in multi-index notation for $\alpha=\left(\alpha_{1}, \ldots, \alpha_{n}\right), \beta=\left(\beta_{1}, \ldots, \beta_{n}\right)$,

$$
u(t)^{n}=(-1)^{n} \sum_{\alpha_{i}+\beta_{i} \geq 1,1 \leq i \leq n} \frac{t^{|\alpha|+|\beta|}}{\alpha ! \beta !} x^{\alpha_{1}} y^{\beta_{1}} \cdots x^{\alpha_{n}} y^{\beta_{n}}
$$

Thus $A$ is a series of Lie brackets with majorant series

$$
\begin{aligned}
& \int_{0}^{1} \sum_{m \geq 0} \frac{t^{m+1}}{(m+1) !}\|X\|^{m}(\|X\|+\|Y\|) \times \\
& \times\left(\frac{1}{2}+\sum_{n \geq 1} \frac{1}{(n+1)(n+2)} \sum_{\alpha_{i}+\beta_{i} \geq 1} \frac{t^{|\alpha|+|\beta|}}{\alpha ! \beta !}\|X\|^{|\alpha|}\|Y\|^{|\beta|}\right) d t .
\end{aligned}
$$

\footnotetext{
${ }^{4}$ If $|$.$| is an arbitrary norm on we have |[X, Y]| \leq M|X||Y|$ for some constant $M$. Then $\|X\|=M|X|$ has the required property.
} 
If $\|X\|+\|Y\|<\log 2$ the latter $\sum$ is $\left(e^{t(\|X\|+\|Y\|)}-1\right)^{n}<1$ and the above integral is less than

$$
\int_{0}^{1} \frac{e^{t\|X\|}-1}{\|X\|} d t(\|X\|+\|Y\|)=\frac{e^{\|X\|}-1-\|X\|}{\|X\|^{2}}(\|X\|+\|Y\|)<\infty,
$$

which implies our claim for $A(X, Y)$. Since $x /\left(e^{-x}-1\right)$ expands as a convergent power series in $x$ for $\|x\|<2 \pi$ we get the same conclusion for $F(X, Y)$.

b. It will be shown in 1.9 that any solution $(F, G)$ of (KV1), e.g. the functions (1.11), also satisfies (KV2) if $\mathfrak{g}$ is a quadratic Lie algebra or (after giving it the additional symmetry $\beta$ introduced in 1.7.2 below) if $\mathfrak{g}$ is solvable. In the latter case the $\beta$-symmetrized (1.11) agrees with the original solution in [30] (see the Appendix to [43]). Unfortunately, in spite of its «natural» look, the above choice of $(F, G)$ does not seem to solve (KV2) for general Lie algebras. This was checked in [1] by computer calculations for the $\beta$-symmetrized (1.11).

c. Assuming (KV1) we have constructed (near the origin; see 1.3 with $t=1$ ) a diffeomorphism $\mathbf{F}$ of $\mathfrak{g} \times \mathfrak{g}$ such that $V \circ \mathbf{F}(X, Y)=X+Y$ with $\mathbf{F}(X, Y)=$ $(f(X, Y) \cdot X, g(X, Y) \cdot Y)$. Setting $a^{-1}=f \circ \mathbf{F}^{-1}, b^{-1}=g \circ \mathbf{F}^{-1}$ we obtain

$$
e^{X} e^{Y}=e^{a(X, Y) \cdot X+b(X, Y) \cdot Y} .
$$

This solves, locally at least, an interesting question in matrix theory (where $\left.a \cdot X=a X a^{-1}, b \cdot Y=b Y b^{-1}\right)$; see e.g. Thompson [51]. Furthermore $b(X, Y)=a(-Y,-X)$ if $(F, G)$ is $\alpha$-invariant, that is $G(X, Y)=F(-Y,-X)$ (see Section 1.7.1).

Besides the Campbell-Hausdorff formula may be written as

$$
\log \left(e^{X} e^{Y}\right)=X+Y+\left(e^{\operatorname{ad} A(X, Y)}-1\right) X+\left(e^{\operatorname{ad} B(X, Y)}-1\right) Y
$$

with $A=\log a, B=\log b$.

d. Assuming (KV1), the trace equation (KV2) is equivalent to the following expression of the Jacobian of the diffeomorphism $\mathbf{F}$ constructed in 1.3:

$$
\operatorname{det}_{\mathfrak{g} \times \mathfrak{g}} D \mathbf{F}(X, Y)=\left(\frac{j(X+Y)}{j(X) j(Y)}\right)^{1 / 2} \operatorname{det}_{\mathfrak{g}} \operatorname{Ad}(f(X, Y) g(X, Y)) .
$$

Let us sketch a proof. By 1.3 the diffeomorphisms $\mathbf{F}_{t}$ solve the differential system $\partial_{t} \mathbf{F}_{t}=u_{t} \circ \mathbf{F}_{t}, \mathbf{F}_{0}=I d$, where $u_{t}$ is the time-dependent vector field on $\mathfrak{g} \times \mathfrak{g}$ defined by $u_{t}(X, Y)=\left(\left[F_{t}(X, Y), X\right],\left[G_{t}(X, Y), Y\right]\right)$. Therefore $\partial_{t} \log \operatorname{det} D \mathbf{F}_{t}=\left(\operatorname{div} u_{t}\right) \circ \mathbf{F}_{t}$ where the divergence div $u_{t}$ is the trace of the Jacobian

$$
D_{(X, Y)} u_{t}=\left(\begin{array}{cc}
\operatorname{ad} F_{t}-x \partial_{X} F_{t} & -x \partial_{Y} F_{t} \\
-y \partial_{X} G_{t} & \operatorname{ad} G_{t}-y \partial_{Y} G_{t}
\end{array}\right)
$$


that is

$$
\partial_{t} \log \operatorname{det} D \mathbf{F}_{t}=\left(\operatorname{tr} \operatorname{ad}\left(F_{t}+G_{t}\right)-\operatorname{tr}\left(x \partial_{X} F_{t}+y \partial_{y} G_{t}\right)\right) \circ \mathbf{F}_{t} .
$$

Observing that $\partial_{t} \log j(t X)=\operatorname{tr}\left(\frac{x}{e^{t x}-1}-\frac{1}{t}\right), \partial_{t} \log \operatorname{det} \operatorname{Ad} f_{t}=\left(\operatorname{tr} \operatorname{ad} F_{t}\right) \circ \mathbf{F}_{t}$ and similarly for $g_{t}$, we obtain

$$
\begin{aligned}
& \partial_{t} \log \left(\left(\frac{j(t X) j(t Y)}{j(t X+t Y)}\right)^{1 / 2} \operatorname{det} \operatorname{Ad}\left(f_{t} g_{t}\right)^{-1} \operatorname{det} D \mathbf{F}_{t}\right)= \\
= & \frac{1}{2} \operatorname{tr}\left(\frac{x}{e^{t x}-1}+\frac{y}{e^{t y}-1}-\frac{x+y}{e^{t(x+y)}-1}-\frac{1}{t}\right)-\operatorname{tr}\left(x \circ \partial_{X} F_{t}+y \circ \partial_{Y} G_{t}\right) \circ \mathbf{F}_{t}
\end{aligned}
$$

and, remembering $X+Y=V_{t} \circ \mathbf{F}_{t}(X, Y)$, our claim easily follows.

\subsubsection{Non-Uniqueness}

The Lie series $F$ and $G$ are not uniquely determined by (KV1) (for instance one may add $\lambda X$ to $F$ and $\mu Y$ to $G$ ), not even by the system (KV1)(KV2) as shown by the next elementary proposition. In 1.7 we shall also give two ways of constructing new solutions of (KV1)(KV2) from a given one.

We need a preliminary lemma.

Lemma 1.7 Let $\mathcal{U}$ be an open subset of $\mathfrak{g} \times \mathfrak{g}$ and $F: \mathcal{U} \rightarrow \mathfrak{g}$ be differentiable, such that $F(g \cdot X, g \cdot Y)=g \cdot F(X, Y)$ whenever $(X, Y) \in \mathcal{U}$ and $(g \cdot X, g \cdot Y) \in \mathcal{U}, g \in G$. Then

$$
\partial_{X} F(X, Y) \circ \operatorname{ad} X+\partial_{Y} F(X, Y) \circ \operatorname{ad} Y=\operatorname{ad} F(X, Y),
$$

as endomorphisms of $\mathfrak{g}$.

Proof. Given $(X, Y) \in \mathcal{U}$ and $Z \in \mathfrak{g}$ we have $(g \cdot X, g \cdot Y) \in \mathcal{U}$ for $g=$ $\exp t Z$ and $|t|$ small enough. Then $g \cdot X=e^{t \operatorname{ad} Z} X$ and the derivative of $F(g \cdot X, g \cdot Y)=g \cdot F(X, Y)$ with respect to $t$ at $t=0$ is

$$
\partial_{X} F(X, Y)[Z, X]+\partial_{Y} F(X, Y)[Z, Y]=[Z, F(X, Y)] .
$$

Proposition 1.8 Given a couple $(F, G)$ of Lie series in $(X, Y)$ and a scalar s let

$$
\begin{array}{lll}
F^{s}(X, Y) & :=F(X, Y)+s(V(X, Y)-X) \\
G^{s}(X, Y) & : \quad=G(X, Y)+s(V(X, Y)-Y) .
\end{array}
$$

Then $(F, G)$ satisfies (KV1), resp. (KV2), if and only if $\left(F^{s}, G^{s}\right)$ satisfies (KV1), resp. (KV2). 
Proof. Since $e^{-x} V=e^{y} V$ we have $\left(1-e^{-x}\right)(V-X)+\left(e^{y}-1\right)(V-Y)=0$ and $(\mathrm{KV} 1)$ for $(F, G)$ is equivalent to $(\mathrm{KV} 1)$ for $\left(F^{s}, G^{s}\right)$. Besides, by the above lemma,

$$
\begin{aligned}
\operatorname{tr}\left(x \circ \partial_{X}(V-X)+y \circ \partial_{Y}(V-Y)\right) & =\operatorname{tr}\left(\partial_{X} V \circ x+\partial_{Y} V \circ y-x-y\right) \\
& =\operatorname{tr}(v-x-y)=0,
\end{aligned}
$$

as explained in 1.4. Thus (KV2) for $(F, G)$ is equivalent to $(\mathrm{KV} 2)$ for $\left(F^{s}, G^{s}\right)$.

We refer to Burgunder [12] $\S 7$ for a systematic study of the non-uniqueness for (KV1); the question boils down to finding all Lie series $A, B \in \mathfrak{l}_{2}$ such that $[X, A]+[Y, B]=0$.

\subsection{An Elementary Proof for $\operatorname{sl}(2, \mathbb{R})$}

In this section ${ }^{5}$ we solve $(\mathrm{KV} 1)$ and $(\mathrm{KV} 2)$ for $\mathfrak{g}=\operatorname{sl}(2, \mathbb{R})$ by an elementary, though lengthy, exercise in linear algebra. The proof uses specific identities valid in this three-dimensional algebra. It does not extend to other examples, whereas Theorem 1.23 below is valid for all quadratic Lie algebras.

This Lie algebra consists of all matrices $X=\left(\begin{array}{cc}a & b \\ c & -a\end{array}\right), a, b, c \in \mathbb{R}$, which we may identify with $X=(a, b, c) \in \mathbb{R}^{3}$. The bracket is

$$
\left[X, X^{\prime}\right]=X X^{\prime}-X^{\prime} X=\left(b c^{\prime}-c b^{\prime}, 2\left(a b^{\prime}-b a^{\prime}\right), 2\left(c a^{\prime}-a c^{\prime}\right)\right),
$$

similar to the clasical vector product in $\mathbb{R}^{3}$. The following facts are easily checked. The Killing form is $(X, Y):=\operatorname{tr}(\operatorname{ad} X \operatorname{ad} Y)=4 \operatorname{tr}(X Y)$, nondegenerate with signature ++- ; in particular $(X, X)=8\left(a^{2}+b c\right)$. Also $\operatorname{tr}$ ad $X=0$ for all $X \in \mathfrak{g}$ since $\operatorname{ad} X$ is skew-symmetric with respect to this form. Besides, with $x=\operatorname{ad} X, y=\operatorname{ad} Y$ as usual,

$$
(x Y, x Y)=([X, Y],[X, Y])=8 \operatorname{det}(X, Y,[X, Y]) .
$$

Thus $X, Y$ and $[X, Y]$ make up a basis of $\mathfrak{g}$ for generic $X, Y$; this is the key to the following calculations. We shall also need the identities

$$
X^{2}=\frac{(X, X)}{8} I
$$

(Cayley-Hamilton theorem) where $I$ is the unit matrix and, for all $X, Y, Z \in$ $\mathfrak{g}$

$$
x y Z=[X,[Y, Z]]=\frac{(X, Y)}{2} Z-\frac{(X, Z)}{2} Y .
$$

In particular

$$
x^{2} Y=-\frac{(X, Y)}{2} X+\frac{(X, X)}{2} Y, y^{2} X=-y x Y=\frac{(Y, Y)}{2} X-\frac{(X, Y)}{2} Y .
$$

\footnotetext{
${ }^{5}$ This section is independent of the sequel and may be skipped.
} 


\subsubsection{Choice of $F$ and $G$}

As in 1.5.2.a we shall make an explicit choice of $A=A(X, Y) \in \mathfrak{g}$ and $B=B(X, Y) \in \mathfrak{g}$, analytic in a neighborhood of the origin in $\mathfrak{g} \times \mathfrak{g}$, such that

$$
W:=\bar{V}-X-Y=[X, A]+[Y, B]
$$

with $\bar{V}=\log \left(e^{Y} e^{X}\right)$. Then $F:=\frac{x}{e^{-x}-1} A, G:=\frac{y}{1-e^{y}} B$ will solve (KV1) and our main task will be to prove $(\mathrm{KV} 2)$. The Campbell-Hausdorff formula may be written as

$$
W=\sum_{|\alpha+\beta| \geq 1} \lambda_{\alpha \beta} y^{\alpha_{1}} x^{\beta_{1}} y^{\alpha_{2}} \cdots y^{\alpha_{k}} x^{\beta_{k}} Y
$$

with rational coefficients $\lambda_{\alpha \beta}, \alpha=\left(\alpha_{1}, \ldots, \alpha_{k}\right), \beta=\left(\beta_{1}, \ldots, \beta_{k}\right) \in \mathbb{N}^{k}$. The series converges absolutely for $\|X\|+\|Y\|<\log 2$ if the norm on $\mathfrak{g}$ is chosen as in 1.5.2. An easy induction with (1.14) shows that each term of the series may be rewritten as $a_{\alpha \beta} x Y$ (if $|\alpha+\beta|$ is odd), $b_{\alpha \beta} x^{2} Y$ or $c_{\alpha \beta} y x Y$ (if $|\alpha+\beta|$ is even), where $a_{\alpha \beta}, b_{\alpha \beta}$ and $c_{\alpha \beta}$ are monomials in the Killing products $(X, X)$, $(X, Y)$ and $(Y, Y)$. Reordering the series we obtain

$$
W=a x Y+b x^{2} Y+c y x Y,
$$

where $a, b$ and $c$ are power series in these products, convergent in a neighborhood of the origin. Since $x$ and $y$ are skew-symmetric it is easily checked that $a, b, c$ satisfy

$$
(x Y, x Y) a=(W, x Y),(x Y, x Y) b=-(W, Y),(x Y, x Y) c=(W, X) .
$$

For any function $f$ on $\mathfrak{g} \times \mathfrak{g}$ we set $\widetilde{f}(X, Y):=f(-Y,-X)$. Because $\log \left(e^{-X} e^{-Y}\right)=-\log \left(e^{Y} e^{X}\right)$ we have $\widetilde{W}=-W$ and (1.16) implies $\widetilde{a}=a$, $\widetilde{b}=-c$ whenever $(x Y, x Y) \neq 0$, or even without this condition since the polynomial function $(x Y, x Y)$ does not vanish identically. Let us choose

$$
\begin{aligned}
A & =\frac{a}{2} Y+b x Y, B=\widetilde{A}=-\frac{a}{2} X+c x Y \\
F & =\frac{x}{e^{-x}-1} A, G=\widetilde{F}=\frac{y}{1-e^{y}} B .
\end{aligned}
$$

Proposition 1.9 Let $\mathfrak{g}=\operatorname{sl}(2, \mathbb{R})$. The above functions $F, G$ are analytic in a neighborhood of the origin in $\mathfrak{g} \times \mathfrak{g}$. They solve (KV1) and (KV2) for $\mathfrak{g}$.

(KV1) follows from (1.15) and (1.17). The proof of

$$
\operatorname{tr}\left(x \circ \partial_{X} F+y \circ \partial_{Y} G\right)=\frac{1}{2} \operatorname{tr}\left(\frac{x}{2} \operatorname{coth} \frac{x}{2}+\frac{y}{2} \operatorname{coth} \frac{y}{2}-\frac{v}{2} \operatorname{coth} \frac{v}{2}-1\right)
$$

(where tr denotes the trace of endomorphisms of $\mathfrak{g}$ ) is given in the next three subsections.

Remark. The above functions $A, B, F, G$ are invariant under the adjoint action of any $g$ in the group $S L(2, \mathbb{R})$, that is $A(g \cdot X, g \cdot Y)=g \cdot A(X, Y)$ etc., because $a(g \cdot X, g \cdot Y)=a(X, Y)$ etc. by invariance of the Killing form. 


\subsubsection{A Variant of (KV2)}

Since $G=\widetilde{F}$ we have $y \circ \partial_{Y} G=\left(x \circ \partial_{X} F\right)^{\sim}$. In the left-hand side of (KV2) we shall thus compute $\operatorname{tr}\left(x \partial_{X} F\right)$ then add its transform under ${ }^{\sim}$. Let

$$
\varphi(x):=\frac{x}{1-e^{-x}}=\frac{x}{2} \operatorname{coth} \frac{x}{2}+\frac{x}{2} .
$$

It is easily checked (details are given in the proof of Lemma $1.25(i)$ ) that

$$
x \partial_{X} F=\varphi(x) \partial_{X}\left(\left(1-e^{-x}\right) F\right)+\operatorname{ad} F+\cdots
$$

where $\cdots$ is a sum of Lie brackets. Remembering (1.17) $\left(1-e^{-x}\right) F=-x A$ and $\operatorname{tr}$ ad $=0$ on $\mathfrak{g}$, we get $\operatorname{tr}\left(x \partial_{X} F\right)=-\operatorname{tr}\left(\varphi(x) \partial_{X}(x A)\right)$.

Let $\bar{v}=\operatorname{ad} \bar{V}$. Since $\partial_{X} \bar{V}=\varphi(\bar{v}) \varphi(x)^{-1}$ by Lemma 1.2 the following observation will shorten the calculations:

$$
\begin{aligned}
\operatorname{tr}\left(\varphi(x) \partial_{X} W\right) & =\operatorname{tr}\left(\left(\partial_{X} \bar{V}-1\right) \varphi(x)\right)=\operatorname{tr}(\varphi(\bar{v})-\varphi(x)) \\
& =\operatorname{tr}\left(\frac{\bar{v}}{2} \operatorname{coth} \frac{\bar{v}}{2}-\frac{x}{2} \operatorname{coth} \frac{x}{2}\right) .
\end{aligned}
$$

But $\bar{V}=\log \left(e^{Y} e^{X}\right)=e^{-x} \log \left(e^{X} e^{Y}\right)=e^{-x} V$, hence $\bar{v}=e^{-x} v e^{x}$ and we may replace $\bar{v}$ by $v=$ ad $V$ under the trace. It follows that

$$
\operatorname{tr}\left(\varphi(x) \partial_{X} W+\left(\varphi(x) \partial_{X} W\right)^{\sim}\right)=\operatorname{tr}\left(v \operatorname{coth} \frac{v}{2}-\frac{x}{2} \operatorname{coth} \frac{x}{2}-\frac{y}{2} \operatorname{coth} \frac{y}{2}\right) .
$$

The similarity with the right-hand side of (KV2) suggests to introduce the function

$$
\begin{aligned}
E & =W-2 x A=-[X, A]+[Y, B]=\left(1-e^{-x}\right) F-\left(e^{y}-1\right) G \\
& =-b x^{2} Y-c y^{2} X,
\end{aligned}
$$

so that (KV2) becomes

$$
\operatorname{tr}\left(\varphi(x) \circ \partial_{X} E+\left(\varphi(x) \circ \partial_{X} E\right)^{\sim}\right)=\operatorname{tr}\left(\frac{v}{2} \operatorname{coth} \frac{v}{2}-1\right) .
$$

Remark. A similar modification of (KV2) will be performed for arbitrary Lie algebras in Section 1.9.

\subsubsection{A Trace Formula for $s l(2, \mathbb{R})$}

Let $u: \mathfrak{g} \rightarrow \mathfrak{g}$ be a linear map. Then for all $X, Y \in \mathfrak{g}$,

$$
(x Y, x Y) \operatorname{tr} u=-\left(u X, y^{2} X\right)-\left(u Y, x^{2} Y\right)+(u x Y, x Y) .
$$

Indeed it suffices to prove this for $(x Y, x Y) \neq 0$; then $X, Y$ and $x Y$ make up a basis of $\mathfrak{g}$. Let $\alpha, \beta, \gamma$ denote the diagonal coefficients of the matrix of $u$ with respect to this basis. Then

$$
\left(u X, y^{2} X\right)=\left(X, y^{2} X\right) \alpha=-(y X, y X) \alpha=-(x Y, x Y) \alpha
$$


since $y$ is skew-symmetric, $y X=-x Y$ and $y^{2} X$ is orthogonal to $Y$ and $x Y$ with respect to the Killing form. Similarly

$$
\begin{aligned}
\left(u Y, x^{2} Y\right) & =\left(Y, x^{2} Y\right)=-(x Y, x Y) \beta \\
(u x Y, x Y) & =(x Y, x Y) \gamma
\end{aligned}
$$

and (1.19) follows for $\operatorname{tr} u=\alpha+\beta+\gamma$.

\subsubsection{Proof of (KV2)}

Again we work for $(x Y, x Y) \neq 0$. By (1.16) and the definition of $E$ we have

$$
E=(W, Y) L-(W, X) M \text { with } L:=\frac{1}{(x Y, x Y)} x^{2} Y, M:=\frac{1}{(x Y, x Y)} y^{2} X .
$$

We first compute $\operatorname{tr}\left(\varphi(x) \partial_{X} E\right)$. Since

$$
\operatorname{tr}\left(\varphi(x) \partial_{X}(f(X) Z)\right)=\left\langle\partial_{X} f(X), \varphi(x) Z\right\rangle
$$

for any scalar function $f$ and constant vector $Z$ (where the brackets $\langle.,$. denote the duality between $\mathfrak{g}^{*}$ and $\mathfrak{g}$ ) we obtain

$$
\begin{aligned}
& \operatorname{tr}\left(\varphi(x) \circ \partial_{X} E\right)=\left\langle\partial_{X}(W, Y), \varphi(x) L\right\rangle-\left\langle\partial_{X}(W, X), \varphi(x) M\right\rangle+ \\
&+(W, Y) \operatorname{tr}\left(\partial_{X} L \circ \varphi(x)\right)-(W, X) \operatorname{tr}\left(\partial_{X} M \circ \varphi(x)\right) .
\end{aligned}
$$

First

$$
\begin{aligned}
\left\langle\partial_{X}(W, Y), \varphi(x) L\right\rangle & =\left(\partial_{X} W \circ \varphi(x) L, Y\right) \\
\left\langle\partial_{X}(W, X), \varphi(x) M\right\rangle & =\left(\partial_{X} W \circ \varphi(x) M, X\right)+(W, \varphi(x) M) .
\end{aligned}
$$

In view of the trace formula (1.19) the last term of (1.20) is given by

$$
\begin{aligned}
& \quad(x Y, x Y) \operatorname{tr}\left(\partial_{X} M \circ \varphi(x)\right)= \\
& =-\left(\partial_{X} M \circ \varphi(x) X, y^{2} X\right)-\left(\partial_{X} M \circ \varphi(x) Y, x^{2} Y\right)+\left(\partial_{X} M \circ \varphi(x) x Y, x Y\right) \\
& =-\left(X,{ }^{t} \partial_{X} M\left(y^{2} X\right)\right)-\left(\varphi(x) Y,{ }^{t} \partial_{X} M\left(x^{2} Y\right)\right)+\left(x \varphi(x) Y,{ }^{t} \partial_{X} M(x Y)\right),
\end{aligned}
$$

where ${ }^{t}$ means transpose with respect to the Killing form and we noted that $\varphi(x) X=\varphi(0) X=X$. But it is easily checked that the products $(X, M)$, $(Y, M)$ and $(x Y, M)$ are constant. Replacing $X$ by $X+t H$ and taking the $t$-derivative at $t=0$ it follows that $(H, M)+\left(X, \partial_{X} M(H)\right)=0$ for all $H \in \mathfrak{g}$, hence ${ }^{t} \partial_{X} M(X)=-M$. Similarly ${ }^{t} \partial_{X} M(Y)=0$ and ${ }^{t} \partial_{X} M(x Y)=-y M$. Finally, using (1.14) and (1.13) with $Z=M$,

$$
(x Y, x Y) \operatorname{tr}\left(\partial_{X} M \circ \varphi(x)\right)=\frac{(X, M)}{2}(Y-\varphi(x) Y, Y) .
$$


The same holds with $M$ replaced by $L$, since the products $(X, L),(Y, L)$ and $(x Y, L)$ are constant. But $(X, L)=0,(X, M)=-1$ and we obtain

$$
\begin{aligned}
(x Y, x Y) \operatorname{tr}\left(\partial_{X} L \circ \varphi(x)\right) & =0 \\
(x Y, x Y) \operatorname{tr}\left(\partial_{X} M \circ \varphi(x)\right) & =\frac{1}{2}(\varphi(x) Y-Y, Y) .
\end{aligned}
$$

Gathering all pieces it follows, with the help of (1.14) and (1.13), that

$$
\begin{aligned}
& (x Y, x Y) \operatorname{tr}\left(\varphi(x) \circ \partial_{X} E\right)= \\
& \quad=\left(\partial_{X} W \circ \varphi(x) x^{2} Y, Y\right)-\left(\partial_{X} W \circ \varphi(x) y^{2} X, X\right)+(W, y x \varphi(x) Y) .
\end{aligned}
$$

As already seen $W=\bar{V}-X-Y$ and $\partial_{X} W \circ \varphi(x)=\varphi(\bar{v})-\varphi(x)$ so that, after some simplifications,

$$
\begin{aligned}
& (x Y, x Y) \operatorname{tr}\left(\varphi(x) \circ \partial_{X} E\right)= \\
& \quad=\left(\varphi(\bar{v}) x^{2} Y, Y\right)-\left(\varphi(\bar{v}) y^{2} X, X\right)+(\bar{V}, y x \varphi(x) Y)-(x Y, x Y) .
\end{aligned}
$$

Adding its $\sim$ to this expression we obtain the left-hand side of (KV2), written as in (1.18). The map changes $\bar{V}$ and $\bar{v}$ into $-\bar{V}$ and $-\bar{v}$. Observing that $\varphi(\bar{v})-\varphi(-\bar{v})=\bar{v}$ and using (1.14) again, the formula boils down to

$$
\begin{aligned}
& (x Y, x Y) \operatorname{tr}\left(\varphi(x) \circ \partial_{X} E+\left(\varphi(x) \circ \partial_{X} E\right)^{\sim}\right)= \\
& \quad=(\bar{V}, y x \varphi(x) Y+x y \varphi(-y) X-(X, Y) x Y)-2(x Y, x Y)
\end{aligned}
$$

after some (easy) manipulations left to the Reader.

Now let $f(x):=\frac{x}{2} \operatorname{coth} \frac{x}{2}=\varphi(x)-\frac{x}{2}$ denote the even part of $\varphi$ and let $\lambda, \mu \in \mathbb{C}$ be such that $\lambda^{2}=(X, X) / 2, \mu^{2}=(Y, Y) / 2$. By (1.14) we have $x^{2 n+1} Y=\lambda^{2 n} x Y$ for $n \geq 0$ and

$$
y x \varphi(x) Y=f(\lambda) y x Y+\frac{1}{2} y x^{2} Y=f(\lambda) y x Y+\frac{(X, Y)}{4} x Y .
$$

A similar transformation of $x y \varphi(-y) X$ shows that the right-hand side of $(1.21)$ is

$$
-\left(\bar{V}, f(\lambda) y^{2} X+f(\mu) x^{2} Y+\frac{(X, Y)}{2} x Y\right)-2(x Y, x Y) .
$$

We must now compute $\bar{V}=\log \left(e^{Y} e^{X}\right)$. For $X \in \mathfrak{g}$ we have $X^{2}=(\lambda / 2)^{2} I$ by (1.12) hence $e^{X}=S(\lambda)(X+f(\lambda) I)$ with $S(\lambda):=\frac{\operatorname{sh} \lambda / 2}{\lambda / 2}$. Introducing $\nu$ such that $\nu^{2}=(\bar{V}, \bar{V}) / 2$ we obtain

$$
\frac{S(\nu)}{S(\lambda) S(\mu)}(\bar{V}+f(\nu) I)=Y X+f(\mu) X+f(\lambda) Y+f(\lambda) f(\mu) I .
$$


The trace is

$$
\frac{S(\nu)}{S(\lambda) S(\mu)} f(\nu)=f(\lambda) f(\mu)+\frac{(X, Y)}{8} .
$$

But the polarized equation (1.12) implies $Y X=\frac{1}{2}[Y, X]+\frac{(X, Y)}{8} I$, therefore

$$
\frac{S(\nu)}{S(\lambda) S(\mu)} \bar{V}=f(\mu) X+f(\lambda) Y-\frac{1}{2} x Y .
$$

Transferring into (1.22) this value of $\bar{V}$ and taking into account various orthogonality relations, (1.23) and (1.21), we finally check that the left-hand side of $(\mathrm{KV} 2)$ is $2(f(\nu)-1)$.

On the other hand we note that, by (1.14),

$$
f(x)-I=(f(\lambda)-1) \frac{x^{2}}{\lambda^{2}}
$$

therefore $\operatorname{tr}(f(x)-I)=2(f(\lambda)-1)$. The right-hand side of (KV2) is then, according to (1.18),

$$
\operatorname{tr}\left(\frac{v}{2} \operatorname{coth} \frac{v}{2}-I\right)=\operatorname{tr}(f(\bar{v})-I)=2(f(\nu)-1),
$$

and (KV2) is proved!

\subsection{Two Symmetries of the Kashiwara-Vergne Problem}

Notation: for any function $f$ of two variables $X, Y$ in a vector space let $^{6}$

$$
f^{\vee}(X, Y):=f(-X,-Y), \bar{f}(X, Y):=f(Y, X), \tilde{f}(X, Y):=f(-Y,-X) .
$$

Together with the identity these three commuting involutions form a group, isomorphic to $(\mathbb{Z} / 2 \mathbb{Z}) \times(\mathbb{Z} / 2 \mathbb{Z})$. For example $V(X, Y)=\log \left(e^{X} e^{Y}\right)$ gives

$$
V^{\vee}=-\bar{V}, \bar{V}=e^{-x} V=e^{y} V, \widetilde{V}=-V .
$$

As usual dots will denote the adjoint action of the group $G$ on its Lie algebra $\mathfrak{g}$.

Let us recall that the solutions of the Kashiwara-Vergne problem can be described by means of $(F, G)$ or $(f, g)$ or $\mathbf{F}$, related as follows (see 1.3): $F$ and $G$ are series of Lie brackets of $X, Y \in \mathfrak{g}$ (near the origin), $f(X, Y)$ and $g(X, Y)$ are elements of the group $G$ obtained by taking $t=1$ in the solutions $f_{t}=f_{t}(X, Y), g_{t}=g_{t}(X, Y)$ of the differential equations

$\partial_{t} f_{t}=\left(D_{e} R_{f_{t}}\right) F_{t}\left(f_{t} \cdot X, g_{t} \cdot Y\right), \partial_{t} g_{t}=\left(D_{e} R_{g_{t}}\right) G_{t}\left(f_{t} \cdot X, g_{t} \cdot Y\right), f_{0}=g_{0}=e$

\footnotetext{
${ }^{6}$ No confusion should arise of the present ${ }^{\sim}$ with the transfer map in 1.1.
} 
with $F_{t}(X, Y)=t^{-1} F(t X, t Y), G_{t}(X, Y)=t^{-1} G(t X, t Y)$, and $\mathbf{F}$ is a diffeomorphism of $\mathfrak{g} \times \mathfrak{g}$ onto itself (near the origin) given by

$$
\mathbf{F}(X, Y):=(f(X, Y) \cdot X, g(X, Y) \cdot Y) .
$$

If $(F, G)$ satisfy (KV1) we have

$$
V \circ \mathbf{F}(X, Y)=V(f \cdot X, g \cdot Y)=X+Y .
$$

Later on we shall also need

$$
E(X, Y):=\left(1-e^{-x}\right) F(X, Y)-\left(e^{y}-1\right) G(X, Y),
$$

motivated by the calculations for $\operatorname{sl}(2, \mathbb{R})$ in 1.6.2.

We now give two constructions of new solutions of the problem from a given one: $(F, G) \mapsto\left(F^{\alpha}, G^{\alpha}\right)$ and $(F, G) \mapsto\left(F^{\beta}, G^{\beta}\right)$; a similar notation is used for their effects on $(f, g)$ and $E$.

\subsubsection{The Symmetry $\alpha$}

Proposition 1.10 Given a couple $(F, G)$ of Lie series, let $\left(F^{\alpha}, G^{\alpha}\right)=(\widetilde{G}, \widetilde{F})$ that is

$$
F^{\alpha}(X, Y):=G(-Y,-X), G^{\alpha}(X, Y):=F(-Y,-X) .
$$

The map $(F, G) \mapsto\left(F^{\alpha}, G^{\alpha}\right)$ is involutive and

$$
\begin{aligned}
f^{\alpha}(X, Y) & =g(-Y,-X), g^{\alpha}(X, Y)=f(-Y,-X) \\
E^{\alpha}(X, Y) & =E(-Y,-X), V\left(f^{\alpha} \cdot X, g^{\alpha} \cdot Y\right)=X+Y .
\end{aligned}
$$

Besides $(F, G)$ satisfies (KV1), resp. (KV2), if and only if $\left(F^{\alpha}, G^{\alpha}\right)$ satisfies (KV1), resp. (KV2).

Proof. All equalities are easily checked. Applying to $(\mathrm{KV} 1)$ for $(F, G)$ we obtain the equivalent relation

$$
\widetilde{\bar{V}}=-\bar{V}=-Y-X-\left(1-e^{y}\right) \widetilde{F}-\left(e^{-x}-1\right) \widetilde{G},
$$

that is $(\mathrm{KV} 1)$ for $\left(F^{\alpha}, G^{\alpha}\right)$. Applying $\sim$ to $(\mathrm{KV} 2)$ for $(F, G)$ (written with coth), we have $\widehat{\partial_{X} F}=-\partial_{Y} \widetilde{F}, \widehat{\partial_{Y} G}=-\partial_{X} \widetilde{G}$ and the right-hand side is invariant since $\widetilde{v}=-v$. It is therefore equivalent to (KV2) for $\left(F^{\alpha}, G^{\alpha}\right)$.

\subsubsection{The Symmetry $\beta$}

Proposition 1.11 Given a couple $(F, G)$ of Lie series, let

$$
\begin{aligned}
& F^{\beta}(X, Y):=e^{x} G(Y, X)+\frac{1}{2}(V(X, Y)-X) \\
& G^{\beta}(X, Y):=e^{-y} F(Y, X)-\frac{1}{2}(V(X, Y)-Y) .
\end{aligned}
$$


The map $(F, G) \mapsto\left(F^{\beta}, G^{\beta}\right)$ is involutive and, if $(F, G)$ satisfies (KV1),

$$
\begin{aligned}
f^{\beta}(X, Y) & =e^{(X+Y) / 2} g(Y, X) e^{-X / 2}, g^{\beta}(X, Y)=e^{-(X+Y) / 2} f(Y, X) e^{Y / 2} \\
E^{\beta}(X, Y) & =-E(Y, X), V\left(f^{\beta} \cdot X, g^{\beta} \cdot Y\right)=X+Y .
\end{aligned}
$$

Besides $(F, G)$ satisfies (KV1), resp. (KV1) and (KV2), if and only if $\left(F^{\beta}, G^{\beta}\right)$ satisfies (KV1), resp. (KV1) and (KV2).

Proof. (i) First

$$
\begin{aligned}
\left(F^{\beta}\right)^{\beta} & =e^{x} \overline{G^{\beta}}+\frac{1}{2}(V-X)=e^{x}\left(e^{-x} F-\frac{1}{2}(\bar{V}-X)\right)+\frac{1}{2}(V-X) \\
& =F
\end{aligned}
$$

since $e^{x} \bar{V}=V$. Similarly $\left(G^{\beta}\right)^{\beta}=G$. Also

$$
\begin{aligned}
E^{\beta} & =\left(1-e^{-x}\right) F^{\beta}-\left(e^{y}-1\right) G^{\beta} \\
& =\left(1-e^{-x}\right)\left(e^{x} \bar{G}+\frac{V}{2}\right)-\left(e^{y}-1\right)\left(e^{-y} \bar{F}-\frac{V}{2}\right) \\
& =\left(e^{x}-1\right) \bar{G}-\left(1-e^{-y}\right) \bar{F}=-\bar{E}
\end{aligned}
$$

since $e^{-x} V=e^{y} V$. Note that (KV1) was not used here.

(ii) Computing as in (i) we obtain

$$
\bar{V}+\left(1-e^{-x}\right) F^{\beta}+\left(e^{y}-1\right) G^{\beta}=V+\overline{\left(1-e^{-x}\right) F+\left(e^{y}-1\right) G},
$$

hence the equivalence of (KV1) for $(F, G)$ and for $\left(F^{\beta}, G^{\beta}\right)$.

(iii) Assuming $(\mathrm{KV} 1)$ for $(F, G)$ we have

$$
e^{y} G=\left(e^{-x}-1\right) F+G-\bar{V}+X+Y
$$

and, exchanging $X$ and $Y$, the definition of $F^{\beta}$ may be written as

$$
F^{\beta}=\left(e^{-y}-1\right) \bar{F}+\bar{G}-\frac{V}{2}+\frac{X}{2}+Y .
$$

Besides

$$
G^{\beta}=\left(e^{-y}-1\right) \bar{F}+\bar{F}-\frac{V}{2}+\frac{Y}{2} .
$$

We may then apply the equality $\left(\partial_{X} S\right) \circ x+\left(\partial_{Y} S\right) \circ y=\operatorname{ad} S$ (Lemma 1.7) to $S=\left(e^{-y}-1\right) \bar{F}$ and $S=V$ hence, noting that $\partial_{X} \bar{G}=\overline{\partial_{Y} G}, \partial_{Y} \bar{F}=\overline{\partial_{X} F}$,

$\partial_{X} F^{\beta} \circ x+\partial_{Y} G^{\beta} \circ y=\overline{\partial_{X} F \circ x+\partial_{Y} G \circ y}+\operatorname{ad}\left(\left(e^{-y}-1\right) \bar{F}-\frac{V-X-Y}{2}\right)$.

Since $\left(e^{-y}-1\right) \bar{F}$ and $V-X-Y$ are sums of brackets it follows that

$$
\operatorname{tr}\left(\partial_{X} F^{\beta} \circ x+\partial_{Y} G^{\beta} \circ y\right)=\operatorname{tr}\left(\overline{\partial_{X} F \circ x+\partial_{Y} G \circ y}\right) .
$$


Assuming (KV2) for $(F, G)$ we now obtain $(\mathrm{KV} 2)$ for $\left(F^{\beta}, G^{\beta}\right)$ : indeed $\operatorname{tr}\left(v /\left(e^{v}-1\right)\right)$ is invariant under $v \mapsto \bar{v}$ since $\bar{V}=e^{-x} V$ and $\bar{v}=e^{-x} v e^{x}$. We conclude that (KV1) and (KV2) for $(F, G)$ imply the same properties for $\left(F^{\beta}, G^{\beta}\right)$ and conversely, $\beta$ being involutive.

(iv) Let $f_{t}^{\beta}(X, Y):=f^{\beta}(t X, t Y), F_{t}^{\beta}(X, Y):=t^{-1} F^{\beta}(t X, t Y)$ and similarly for $g_{t}^{\beta}, G_{t}^{\beta}$. We claim that

$$
\begin{aligned}
\left(f_{t}^{\beta}\right)^{\prime}\left(f_{t}^{\beta}\right)^{-1} & =F_{t}^{\beta}\left(f_{t}^{\beta} \cdot X, g_{t}^{\beta} \cdot Y\right), f_{0}^{\beta}=e \\
\left(g_{t}^{\beta}\right)^{\prime}\left(g_{t}^{\beta}\right)^{-1} & =G_{t}^{\beta}\left(f_{t}^{\beta} \cdot X, g_{t}^{\beta} \cdot Y\right), g_{0}^{\beta}=e
\end{aligned}
$$

if $f^{\beta}, F^{\beta}$ etc. are defined as in the proposition. Here' denotes the derivative with respect to $t$ and $f^{\prime} f^{-1}$ means $\left(D_{e} R_{f}\right)^{-1} f^{\prime}$ for the sake of simplicity. Replacing $(X, Y)$ by $(t X, t Y)$ it will suffice to prove (1.25) for $t=1$. Details are given for $f^{\beta}$ below; the proof for $g^{\beta}$ is similar.

If $a, b, c$ are differentiable maps from an interval of $\mathbb{R}$ into $G$ we have the following formulas for «logarithmic derivatives» (in our simplified notation)

$$
\begin{aligned}
(a b)^{\prime}(a b)^{-1} & =\left(a^{\prime} a^{-1}\right)+a \cdot\left(b^{\prime} b^{-1}\right) \\
(a b c)^{\prime}(a b c)^{-1} & =\left(a^{\prime} a^{-1}\right)+a \cdot\left(b^{\prime} b^{-1}\right)+(a b) \cdot\left(c^{\prime} c^{-1}\right),
\end{aligned}
$$

where dots denote the adjoint action of $G$ on its Lie algebra. Applying this to

$$
f_{t}^{\beta}=e^{t(X+Y) / 2} \overline{g_{t}} e^{-t X / 2}, g_{t}^{\beta}=e^{-t(X+Y) / 2} \overline{f_{t}} e^{t Y / 2}
$$

we obtain, for $t=1$,

$$
\left(f_{t}^{\beta}\right)^{\prime}\left(f^{\beta}\right)^{-1}=\frac{X+Y}{2}+e^{(X+Y) / 2} \cdot\left(\overline{g_{t}}\right)^{\prime}(\bar{g})^{-1}-\left(e^{(X+Y) / 2} \bar{g}\right) \cdot \frac{X}{2} .
$$

The claimed equality (1.25) for $\left(f_{t}^{\beta}\right)^{\prime}\left(f^{\beta}\right)^{-1}$ now ensues from the following remarks $(a)$ and $(b)$ :

(a) $X+Y=V\left(f^{\beta} \cdot X, g^{\beta} \cdot Y\right)$. Indeed $f^{\beta} \cdot X=e^{(X+Y) / 2} \cdot(\bar{g} \cdot X)$ and $g^{\beta} \cdot Y=e^{-(X+Y) / 2} \cdot(\bar{f} \cdot Y)$, hence

$$
\begin{aligned}
\exp V\left(f^{\beta} \cdot X, g^{\beta} \cdot Y\right) & =\exp \left(f^{\beta} \cdot X\right) \exp \left(g^{\beta} \cdot Y\right) \\
& =e^{(X+Y) / 2}\left(e^{\bar{g} \cdot X} e^{-(X+Y)} e^{\bar{f} \cdot Y}\right) e^{(X+Y) / 2} .
\end{aligned}
$$

But $X+Y=V(f \cdot X, g \cdot Y)$ by (KV1) (see Section 1.3), and this implies $Y+X=V(\bar{f} \cdot Y, \bar{g} \cdot X)$ therefore $e^{X+Y}=e^{\bar{f} \cdot Y} e^{\bar{g} \cdot X}$ and $\exp V\left(f^{\beta} \cdot X, g^{\beta}\right.$. $Y)=\exp (X+Y)$

(b) $g_{t}^{\prime} g^{-1}=G(f \cdot X, g \cdot Y)$ hence $\left(\overline{g_{t}}\right)^{\prime}(\bar{g})^{-1}=G(\bar{f} \cdot Y, \bar{g} \cdot X)=\bar{G}(\bar{g} \cdot X, \bar{f} \cdot Y)$. Also

$$
e^{(X+Y) / 2} \cdot \bar{G}(\bar{g} \cdot X, \bar{f} \cdot Y)=\bar{G}\left(\left(e^{(X+Y) / 2} \bar{g}\right) \cdot X,\left(e^{(X+Y) / 2} \bar{f}\right) \cdot Y\right) .
$$


Observing that $\left(e^{(X+Y) / 2} \bar{g}\right) \cdot X=f^{\beta} \cdot X$ and, by $(a)$,

$$
\begin{aligned}
\left(e^{(X+Y) / 2} \bar{f}\right) \cdot Y & =\left(e^{X+Y} g^{\beta}\right) \cdot Y=\left(e^{f^{\beta} \cdot X} e^{g^{\beta} \cdot Y} g^{\beta}\right) \cdot Y \\
& =e^{f^{\beta} \cdot X} \cdot\left(g^{\beta} \cdot Y\right),
\end{aligned}
$$

we infer that

$$
e^{(X+Y) / 2} \cdot\left(\overline{g_{t}}\right)^{\prime}(\bar{g})^{-1}=e^{f^{\beta} \cdot X} \cdot \bar{G}\left(f^{\beta} \cdot X, g^{\beta} \cdot Y\right) .
$$

From (a) and (b) it follows that (1.27) may be written as

$$
\begin{aligned}
\left(f_{t}^{\beta}\right)^{\prime}\left(f^{\beta}\right)^{-1} & =\frac{1}{2} V\left(f^{\beta} \cdot X, g^{\beta} \cdot Y\right)+e^{f^{\beta} \cdot X} \cdot \bar{G}\left(f^{\beta} \cdot X, g^{\beta} \cdot Y\right)-\frac{1}{2} f^{\beta} \cdot X \\
& =F^{\beta}\left(f^{\beta} \cdot X, g^{\beta} \cdot Y\right),
\end{aligned}
$$

which completes the proof.

Remark. A similar proof to (iv) shows that the couple $\left(F^{s}, G^{s}\right)$ of Proposition 1.8 corresponds to $\left(f^{s}, g^{s}\right)$ given by

$$
f^{s}(X, Y)=e^{s(X+Y)} f(X, Y) e^{-s X}, g^{s}(X, Y)=e^{s(X+Y)} g(X, Y) e^{-s Y},
$$

and $E^{s}=E+2 s(V-\bar{V}), V\left(f^{s} \cdot X, g^{s} \cdot Y\right)=X+Y$.

\subsubsection{Symmetry Invariant Solutions}

We keep to the notation of 1.7.1 and 1.7.2.

Proposition 1.12 (i) The above symmetries $\alpha$ and $\beta$ are commuting involutions acting on couples of Lie series in $(X, Y)$.

(ii) For any solution $(F, G)$ of (KV1), resp. (KV1) and (KV2), the couple

$$
\left(F_{0}, G_{0}\right)=\frac{1}{4}\left(F+F^{\alpha}+F^{\beta}+F^{\alpha \beta}, G+G^{\alpha}+G^{\beta}+G^{\alpha \beta}\right)
$$

is a solution of (KV1), resp. (KV1) and (KV2), invariant under $\alpha$ and $\beta$. Explicitly

$$
\begin{aligned}
F_{0}(X, Y) & =\frac{1}{4}(F(X, Y)+G(-Y,-X))+\frac{1}{4} e^{x}(F(-X,-Y)+G(Y, X))+ \\
& +\frac{1}{4}(V(X, Y)-X) \\
G_{0}(X, Y) & =F_{0}(-Y,-X) .
\end{aligned}
$$

Proof. (i) The definitions imply

$$
\begin{aligned}
& \left(F^{\alpha}\right)^{\beta}=e^{x} \overline{G^{\alpha}}+\frac{1}{2}(V-X)=e^{x} F^{\vee}+\frac{1}{2}(V-X) \\
& \left(F^{\beta}\right)^{\alpha}=\widetilde{G^{\beta}}=\left(e^{-y} \bar{F}-\frac{1}{2}(V-Y)\right) \sim=e^{x} F^{\vee}-\frac{1}{2}(\widetilde{V}+X),
\end{aligned}
$$


and $\widetilde{V}=-V$ gives $\left(F^{\alpha}\right)^{\beta}=\left(F^{\beta}\right)^{\alpha}$. Similarly $\left(G^{\alpha}\right)^{\beta}=\left(G^{\beta}\right)^{\alpha}$.

(ii) is clear from Propositions 1.10, 1.11 and the linearity of the KashiwaraVergne equations.

Proposition 1.13 (i) A couple $(F, G)$ of Lie series in $(X, Y)$ is invariant under $\alpha$ and $\beta$ if and only if there exists an even Lie series $H(X, Y)$ such that

$$
\begin{aligned}
& F(X, Y)=e^{x / 2} H(X, Y)+\frac{1}{4}(V(X, Y)-X) \\
& G(X, Y)=e^{-y / 2} H(Y, X)-\frac{1}{4}(V(X, Y)-Y) .
\end{aligned}
$$

In this case we have $G(X, Y)=F(-Y,-X)$ and $E(X, Y)=-E(-X,-Y)=$ $-E(Y, X)$. Besides $F=(Y / 4)+\cdots, G=-(X / 4)+\cdots$, where dots are sums of brackets of $X$ and $Y$.

(ii) Let $(F, G)$ be a solution of (KV1) invariant under $\alpha$ and $\beta$. Then the corresponding elements $f, g$ of the group satisfy

$$
f(X, Y)=e^{(X+Y) / 4} u(X, Y) e^{-X / 4}, g(X, Y)=f(-Y,-X)
$$

where $u(X, Y) \in G$ is an even function of $(X, Y)$.

Proof. (i) The invariance $\left(F^{\alpha}, G^{\alpha}\right)=(F, G)$ is equivalent to $G=\widetilde{F}$ that is $\bar{G}=F^{\vee}$. Let us write $e^{-x / 2} F=H+K$ where $H$ is even and $K$ is odd. Then, assuming the $\alpha$-invariance, the $\beta$-invariance $F=F^{\beta}$ gives $F=$ $e^{x} F^{\vee}+\frac{1}{2}(V-X)$ that is

$$
e^{x / 2}(H+K)=e^{x} e^{-x / 2}(H-K)+\frac{1}{2}(V-X)
$$

which boils down to $K=\frac{1}{4} e^{-x / 2}(V-X)$ hence $F=e^{x / 2} H+(V-X) / 4$. Conversely, this together with $G=\widetilde{F}$ ensures the $\alpha$ - and $\beta$-invariance of $(F, G)$ since $\widetilde{V}=-V$. The corresponding properties of $E$ follow from Propositions 1.10 and 1.11. Besides $V=X+Y+\cdots$ where dots are brackets of $X, Y$ and, $H$ being an even Lie series, $H=a[X, Y]+\cdots$ Thus $F(X, Y)=(Y / 4)+\cdots$ and $G(X, Y)=F(-Y,-X)=-(X / 4)+\cdots$.

(ii) As for (i) $f^{\alpha}=f$ gives $g=\widetilde{f}$ that is $\bar{g}=f^{\vee}$. Then $f=f^{\beta}$ implies $f=$ $e^{(X+Y) / 2} f^{\vee} e^{-X / 2}$ by Proposition 1.11 , which means that $e^{-(X+Y) / 4} f e^{X / 4}$ is even.

\subsection{From (KV1) Towards (KV2)}

In their 2009 note [8], using the free Lie algebra formalism they had developed in [7], Alekseev and Torossian discovered a remarkable link between equations (KV1) and (KV2). In this section we extract from their work the 
necessary material, eventually leading in 1.9 to a proof of the conjecture in two important cases.

Let $\mathbb{K}$ be a commutative field of characteristic zero. We work here with the completed free Lie algebra $\mathfrak{l}_{2}$ introduced in 1.5.1 and with $\mathcal{A}_{2}$, the completion (with respect to the degree) of the free associative algebra with two generators $X, Y$ over $\mathbb{K}$, that is the space of all associative but non-commutative formal series in $X$ and $Y$. Any element $a \in \mathcal{A}_{2}$ has a unique decomposition

$$
a=a^{0}+a^{X} X+a^{Y} Y
$$

with $a^{0} \in \mathbb{K}$ and $a^{X}, a^{Y} \in \mathcal{A}_{2}$; for example $[X, Y] \in \mathfrak{l}_{2} \subset \mathcal{A}_{2}$ decomposes as $[X, Y]=0+(-Y) X+X Y$. For $a \in \mathfrak{l}_{2}$ the components $a^{X}$ and $a^{Y}$ play the role of «partial derivatives» of $a$ with respect to $X$ and $Y$; see Lemma 1.21 for a precise statement.

Let $\tau$ denote the anti-involution of $\mathcal{A}_{2}$ defined by $\tau(X)=-X$ and $\tau(Y)=$ $-Y$. Thus $\tau(a b)=\tau(b) \tau(a)$ for all $a, b \in \mathcal{A}_{2}, \tau^{2}$ is the identity and $\tau(Z)=$ $-Z$ for all $Z \in \mathfrak{l}_{2}$. The goal of this section is to prove the following theorem.

Theorem 1.14 Let $F, G \in \mathfrak{l}_{2}$ be two Lie series in $(X, Y)$ such that (KV1) holds. Then there exists $a \in \mathcal{A}_{2}$ such that $\tau(a)=-a$ and

$$
F^{X} X+G^{Y} Y \sim \frac{1}{2}\left(\frac{X}{2} \operatorname{coth} \frac{X}{2}+\frac{Y}{2} \operatorname{coth} \frac{Y}{2}-\frac{V}{2} \operatorname{coth} \frac{V}{2}-1\right)+a
$$

where $V=V(X, Y)$ and $\sim$ means equal modulo $\left[\mathcal{A}_{2}, \mathcal{A}_{2}\right]$.

The link between this property and (KV2) will appear in Section 1.9. The proof of Theorem 1.14 requires several formal tools which we now introduce as we need them. We divide it into seven steps.

\subsubsection{Tangential Derivations and Automorphisms}

As shown in 1.3 for a finite-dimensional Lie algebra $\mathfrak{g}$, the main role of Lie series $F, G$ satisfying (KV1) is to allow constructing a one-parameter family of local diffeomorphisms $\mathbf{F}_{t}$ of $\mathfrak{g} \times \mathfrak{g}$ given by the adjoint action of elements $f_{t}=f_{t}(X, Y), g_{t}=g_{t}(X, Y)$ of $G$, namely $\mathbf{F}_{t}(X, Y)=\left(f_{t} \cdot X, g_{t} \cdot Y\right)$, and such that $V_{t}\left(\mathbf{F}_{t}(X, Y)\right)=X+Y$. Our first goal is to introduce similar tools in the context of the free Lie algebra $\mathfrak{l}_{2}$ with generators $X, Y$. Observing that $F, G \in \mathfrak{l}_{2}$ were only used by means of the brackets $[X, F]$ and $[Y, G]$ we are led to the following definition.

Let $\mathbb{K}$ be a commutative field of characteristic zero and $\mathfrak{l}_{n}$ be the completed free Lie algebra with $n$ generators $X_{1}, \ldots, X_{n}$ over $\mathbb{K}$. A derivation $D$ of $\mathfrak{l}_{n}$ is determined by its values $D\left(X_{i}\right)$ on the generators. It is called a tangential derivation if there exist $D_{1}, \ldots, D_{n} \in \mathfrak{l}_{n}$ such that

$$
D\left(X_{i}\right)=\left[X_{i}, D_{i}\right], i=1, \ldots, n .
$$


Remark. Such a $D_{i}$ is only unique up to the addition of some multiple of $X_{i}$. In the sequel we shall assume that, for each $i, D_{i}$ has no $X_{i}$-component among its first order terms. The map $\left(D_{1}, \ldots, D_{n}\right) \mapsto D$ thus becomes a bijection onto the set of tangential derivations and we may simply write $D=\left(D_{1}, \ldots, D_{n}\right)$.

Under the natural bracket of linear operators the tangential derivations of $\mathfrak{l}_{n}$ form a Lie algebra, denoted by $\mathfrak{t}_{n}$. Indeed an easy computation shows that $\left[D, D^{\prime}\right]=D^{\prime \prime}$ with $D_{i}^{\prime \prime}=\left[D_{i}, D_{i}^{\prime}\right]+D\left(D_{i}^{\prime}\right)-D^{\prime}\left(D_{i}\right)$.

The algebra $\mathfrak{t a}_{n}$ carries a natural grading induced by the one in $\mathfrak{l}_{n}$. For all $k=1,2, \ldots$ the subspace of elements of degree $k$ is finite-dimensional. We can therefore associate a group, denoted by $T A_{n}$, to this Lie algebra. As a set it is $\mathfrak{t}_{n}$, equipped with the group law defined by the Campbell-Hausdorff series $V$ : writing $\exp : \mathfrak{t}_{n} \rightarrow T A_{n}$ the map identitying both sets, we have $\exp D \exp D^{\prime}:=\exp V\left(D, D^{\prime}\right)$ for $D, D^{\prime} \in \mathfrak{t o}_{n}$. Each element $g$ of $T A_{n}$ is $g=\exp D$ for some $D \in \mathfrak{t a}_{n}$, and defines an automorphism of $\mathfrak{l}_{n}$ according to

$$
g(Z)=\sum_{k=0}^{\infty} \frac{1}{k !} D^{k}(Z), Z \in \mathfrak{l}_{n} .
$$

$T A_{n}$ is called the group of tangential automorphisms.

Introducing a parameter $t$, let $\left(\mathcal{F}_{t}\right)$ be a one-parameter family of automorphisms of $\mathfrak{l}_{n}$ such that $\mathcal{F}_{t}(Z)$ belongs to $\mathfrak{l}_{n}[[t]]:=\mathfrak{l}_{n} \otimes_{\mathbb{K}} \mathbb{K}[[t]]$ for any $Z \in \mathfrak{l}_{n}$. In other words $\mathcal{F}_{t}(Z)$ expands as a series of homogeneous elements of $\mathfrak{l}_{n}$, with formal power series in $t$ as the coefficients. Taking the derivative of the coefficients with respect to $t$ we obtain a linear map from $\mathfrak{l}_{n}$ into itself denoted by $\partial_{t} \mathcal{F}$. Then $\mathcal{F}_{t}^{-1} \partial_{t} \mathcal{F}$ is a one-parameter family of derivations of $\mathfrak{l}_{n}$, as seen by taking the derivative of $\mathcal{F}_{t}\left(\left[Z_{1}, Z_{2}\right]\right)=\left[\mathcal{F}_{t}\left(Z_{1}\right), \mathcal{F}_{t}\left(Z_{2}\right)\right]$.

Conversely let $D_{t}=\left(D_{t, 1}, \ldots, D_{t, n}\right)$ be a family of tangential derivations, with $D_{t, i} \in \mathfrak{l}_{n}[[t]]$. The differential equation

$$
\mathcal{F}_{t}^{-1} \partial_{t} \mathcal{F}_{t}=-D_{t}, \mathcal{F}_{0}=e
$$

defines a one-parameter family of tangential automorphisms $\mathcal{F}_{t} \in T A_{n}$, as we now explain.

Indeed let $A_{i}=A_{i}(t) \in \mathfrak{l}_{n}[[t]]$ be the solution of the differential system

$$
\partial_{t} A_{i}=\frac{\operatorname{ad} A_{i}}{e^{\text {ad } A_{i}}-1} D_{t, i}\left(e^{\text {ad } A_{1}} X_{1}, \ldots, e^{\text {ad } A_{n}} X_{n}\right), A_{i}(0)=0,
$$

where $D_{t, i}\left(e^{\text {ad } A_{1}} X_{1}, \ldots\right)$ is obtained from $D_{t, i}$ by replacing the generators $X_{i}$ by $e^{\text {ad } A_{i}} X_{i}$. Repeating a classical proof for the differential of exp, we set $Z_{i}(s, t):=e^{s \text { ad } A_{i}(t)} X_{i}$. Then $\partial_{s} Z_{i}=\left[A_{i}, Z_{i}\right]$ hence

$$
\begin{aligned}
\partial_{s}\left(e^{-s \operatorname{ad} A_{i}(t)} \partial_{t} Z_{i}(s, t)\right) & =\left[e^{-s \operatorname{ad} A_{i}(t)} \partial_{t} A_{i}(t), X_{i}\right] \\
& =\partial_{s}\left[\frac{1-e^{-s \operatorname{ad} A_{i}(t)}}{\operatorname{ad} A_{i}(t)} \partial_{t} A_{i}(t), X_{i}\right] .
\end{aligned}
$$


Since $\partial_{t} Z_{i}(0, t)=0$ we infer that, for $s=1$,

$$
e^{-\mathrm{ad} A_{i}(t)} \partial_{t} Z_{i}(1, t)=\left[\frac{1-e^{-\operatorname{ad} A_{i}(t)}}{\operatorname{ad} A_{i}(t)} \partial_{t} A_{i}(t), X_{i}\right] .
$$

Setting $\mathcal{F}_{t}\left(X_{i}\right):=Z_{i}(1, t)=e^{\text {ad } A_{i}(t)} X_{i}$, the expression of $\partial_{t} A_{i}$ finally leads to

$$
\partial_{t} \mathcal{F}_{t}\left(X_{i}\right)=\left[D_{t, i}\left(\mathcal{F}_{t}\left(X_{1}\right), \ldots, \mathcal{F}_{t}\left(X_{n}\right)\right), \mathcal{F}_{t}\left(X_{i}\right)\right]=-\mathcal{F}_{t}\left(D_{t}\left(X_{i}\right)\right) .
$$

Thus $\mathcal{F}_{t}$ defines the sought after tangential automorphism of $\mathfrak{l}_{n}$.

\subsubsection{Behavior Under Scaling Maps}

For $t \in \mathbb{K} \backslash 0$ let $\Lambda_{t}$ denote the scaling automorphism of the Lie algebra $\mathfrak{l}_{n}$ defined by $\Lambda_{t}\left(X_{i}\right):=t X_{i}$ for $i=1, \ldots, n$. Thus, for any Lie series $F=$ $F\left(X_{1}, \ldots, X_{n}\right) \in \mathfrak{l}_{n}$ we have

$$
\left(\Lambda_{t} F\right)\left(X_{1}, \ldots, X_{n}\right)=F\left(t X_{1}, \ldots, t X_{n}\right) .
$$

Since $F$ has no zero order term we may introduce $F_{t}=t^{-1} \Lambda_{t} F$, that is $F_{t}\left(X_{1}, \ldots, X_{n}\right)=t^{-1} F\left(t X_{1}, \ldots, t X_{n}\right)$. Then $F_{t} \in \mathfrak{l}_{n}[[t]], F_{1}=F$ and $\Lambda_{t} F=$ $t F_{t}$ for $t \in \mathbb{K} \backslash 0$.

Lemma 1.15 Given a tangential derivation $D=\left(D_{1}, \ldots, D_{n}\right) \in \mathfrak{t a}_{n}$ and $t \in \mathbb{K} \backslash 0$, let $D_{t}:=t^{-1} \Lambda_{t} D \Lambda_{t}^{-1}$. Then $D_{t}$ is a tangential derivation and the solution $\mathcal{F}_{t} \in T A_{n}$ of the differential equation (1.29) $\mathcal{F}_{t}^{-1} \partial_{t} \mathcal{F}_{t}=-D_{t}$, $\mathcal{F}_{0}=e$ satisfies $\mathcal{F}_{t}=\Lambda_{t} \mathcal{F} \Lambda_{t}^{-1}$ with $\mathcal{F}=\mathcal{F}_{1}$.

Proof. The first assertion is easy:

$$
D_{t}\left(X_{i}\right)=t^{-1} \Lambda_{t}\left[t^{-1} X_{i}, D_{i}\right]=t^{-1}\left[X_{i}, \Lambda_{t}\left(D_{i}\right)\right]=\left[X_{i}, D_{t, i}\right]
$$

with $D_{t, i}=t^{-1} \Lambda_{t}\left(D_{i}\right) \in \mathfrak{l}_{n}[[t]]$.

The second follows from the uniqueness of solutions of (1.29). Indeed ${ }_{1} \mathcal{G}_{t}:=$ $\mathcal{F}_{s t}$ and ${ }_{2} \mathcal{G}_{t}:=\Lambda_{s} \mathcal{F}_{t} \Lambda_{s}^{-1}$ are, for fixed $s$, two solutions of the differential system

$$
\mathcal{G}_{t}^{-1} \partial_{t} \mathcal{G}_{t}=-s D_{s t}, \mathcal{G}_{0}=e
$$

since $s D_{s t}=\Lambda_{s} D_{t} \Lambda_{s}^{-1}$. Thus $\mathcal{F}_{s t}=\Lambda_{s} \mathcal{F}_{t} \Lambda_{s}^{-1}$ and $t=1$ implies our claim.

Specializing to $n=2$, let $F, G \in \mathfrak{l}_{2}$ such that (KV1) holds. The argument given in the Lie algebra $\mathfrak{g}$ (Section 1.3) remains valid in the free algebra $\mathfrak{l}_{2}$ and gives

$$
\partial_{t} V_{t}(X, Y)=D_{t} V_{t}(X, Y)
$$

where $D_{t} \in \mathfrak{t}_{2}$ is the tangential derivation defined by $D_{t}=\left(F_{t}, G_{t}\right)$. Then $\mathcal{F}_{t}^{-1} \partial_{t} \mathcal{F}_{t}=-D_{t}$ implies

$$
\begin{aligned}
\partial_{t}\left(\mathcal{F}_{t}\left(V_{t}\right)\right) & =\mathcal{F}_{t}\left(\partial_{t} V_{t}\right)+\left(\partial_{t} \mathcal{F}_{t}\right)\left(V_{t}\right) \\
& =\mathcal{F}_{t}\left(\partial_{t} V_{t}-D_{t} V_{t}\right)=0,
\end{aligned}
$$


hence $\mathcal{F}_{t}\left(V_{t}(X, Y)\right)=\mathcal{F}_{0}\left(V_{0}(X, Y)\right)$, that is

$$
\mathcal{F}_{t}\left(V_{t}(X, Y)\right)=X+Y .
$$

Remark. Note the change of notation between $\mathfrak{g}$ and $\mathfrak{l}_{2}$ : in the former (see 1.3) $\mathbf{F}_{t}$ was a diffeomorphism of $\mathfrak{g} \times \mathfrak{g}$ (near the origin) whereas, in the latter, $\mathcal{F}_{t}$ denotes a map from $\mathfrak{l}_{2}$ into itself. If $X, Y$ belong to $\mathfrak{g}$ they are linked by $\mathbf{F}_{t}(X, Y)=\left(\mathcal{F}_{t}(X), \mathcal{F}_{t}(Y)\right)$. Accordingly the property $V_{t}\left(\mathbf{F}_{t}(X, Y)\right)=X+Y$ is replaced here by

$$
V_{t}\left(\mathcal{F}_{t}(X), \mathcal{F}_{t}(Y)\right)=\mathcal{F}_{t}\left(V_{t}(X, Y)\right)=X+Y,
$$

where the first equality holds because $V_{t}$ is a Lie series in $X, Y$ and $\mathcal{F}_{t}$ is an automorphism of $\mathfrak{l}_{2}$.

\subsubsection{Using Associativity}

A key observation in the proof of Theorem 1.14 is the associativity

$$
V(V(X, Y), Z)=V(X, V(Y, Z))
$$

satisfied by the Campbell-Hausdorff law, a consequence of the associativity of the group law and the definition of $V$. To exploit it we now work in the (completed) free Lie algebra $\mathfrak{l}_{3}$ with generators $X, Y, Z$. Since $\mathcal{F}(V(X, Y))=$ $X+Y$ by (1.30) we have successively

$$
\begin{aligned}
\mathcal{F}_{1,2}(V(V(X, Y), Z)) & =V(X+Y, Z) \\
\mathcal{F}_{12,3} \mathcal{F}_{1,2}(V(V(X, Y), Z)) & =(X+Y)+Z
\end{aligned}
$$

where $\mathcal{F}_{1,2} \in T A_{3}$ means $\mathcal{F}$ acting on the first and second generators of $\mathfrak{l}_{3}$, trivially on the third, and $\mathcal{F}_{12,3} \in T A_{3}$ means $\mathcal{F}$ acting on $X+Y$ and $Z$. Similarly

$$
\begin{aligned}
\mathcal{F}_{2,3}(V(X, V(Y, Z))) & =V(X, Y+Z) \\
\mathcal{F}_{1,23} \mathcal{F}_{2,3}(V(X, V(Y, Z))) & =X+(Y+Z) .
\end{aligned}
$$

Introducing the automorphism $\Phi \in T A_{3}$ defined by

$$
\Phi \mathcal{F}_{1,23} \mathcal{F}_{2,3}=\mathcal{F}_{12,3} \mathcal{F}_{1,2}
$$

we see that the associativity of $V$ implies

$$
\Phi(X+Y+Z)=X+Y+Z .
$$

This special property of $\Phi$, related to $\mathcal{F}$ hence to $(F, G)$, will eventually lead to the conclusion. 


\subsubsection{A «Divergence» and a «Jacobian»}

In $\mathcal{A}_{n}$, the completion of the free associative algebra with generators $X_{1}, . ., X_{n}$ over $\mathbb{K}$, we denote by

$$
a=a^{0}+\sum_{k=1}^{n} a^{k} X_{k}
$$

the unique decomposition of any $a \in \mathcal{A}_{n}$ with $a^{0} \in \mathbb{K}$ and $a^{k} \in \mathcal{A}_{n}$.

Let $\tau$ denote the anti-involution of $\mathcal{A}_{n}$ defined by $\tau\left(X_{i}\right)=-X_{i}, i=$ $1, \ldots, n$. Thus $\tau(a b)=\tau(b) \tau(a)$ for all $a, b \in \mathcal{A}_{n}, \tau^{2}$ is the identity map and $\tau(Z)=-Z$ for all $Z \in \mathfrak{l}_{n}$. Let $\mathcal{N}_{n}=\operatorname{ker}(1+\tau)$ be the $(-1)$-eigenspace of $\tau$ in $\mathcal{A}_{n}$.

Let $\left\langle\mathcal{A}_{n}\right\rangle:=\mathcal{A}_{n}^{+} /\left(\left[\mathcal{A}_{n}, \mathcal{A}_{n}\right]+\mathcal{N}_{n}\right)$ be the quotient of $\mathcal{A}_{n}^{+}$(the subalgebra of elements of degree $\geq 1$ ) by the space of all linear combinations of brackets in $\mathcal{A}_{n}$ and of elements of $\mathcal{N}_{n}$, and let $a \mapsto\langle a\rangle$ denote $^{7}$ the canonical projection $\mathcal{A}_{n} \rightarrow\left\langle\mathcal{A}_{n}\right\rangle$. One can think of $\mathcal{A}_{n}^{+} /\left[\mathcal{A}_{n}, \mathcal{A}_{n}\right]$ as the $\mathbb{K}$-vector space spanned by cyclic words in the letters $X_{1}, \ldots, X_{n}$; including $\mathcal{N}_{n}$ in the quotient will play no role until Proposition 1.17.

Any derivation $D$ of the Lie algebra $\mathfrak{l}_{n}$ extends to a derivation of the associative algebra $\mathcal{A}_{n}$, and descends to the graded vector space $\left\langle\mathcal{A}_{n}\right\rangle$. Indeed $D\left(\left[\mathcal{A}_{n}, \mathcal{A}_{n}\right]\right) \subset\left[\mathcal{A}_{n}, \mathcal{A}_{n}\right]$ and $\tau D \tau^{-1}$ is a derivation of $\mathcal{A}_{n}$ such that $\tau D \tau^{-1}\left(X_{i}\right)=-\tau D\left(X_{i}\right)=D\left(X_{i}\right)$; thus $\tau D \tau^{-1}=D$ hence $D \mathcal{N}_{n} \subset \mathcal{N}_{n}$. We denote by $D \cdot\langle a\rangle$ this action of $D$ on $\langle a\rangle \in\left\langle\mathcal{A}_{n}\right\rangle$.

Similarly tangential and scaling automorphisms of $\mathfrak{l}_{n}$ extend to automorphisms of $\mathcal{A}_{n}$ and descend to $\left\langle\mathcal{A}_{n}\right\rangle$, since they map $\left[\mathcal{A}_{n}, \mathcal{A}_{n}\right]$ into itself and commute with $\tau$.

The «divergence» is the map div : $\mathfrak{t a}_{n} \rightarrow\left\langle\mathcal{A}_{n}\right\rangle$ defined by

$$
\operatorname{div} D:=\left\langle\sum_{i=1}^{n} D_{i}^{i} X_{i}\right\rangle
$$

where $D=\left(D_{1}, \ldots, D_{n}\right)$ (see Remark in 1.8.1) and $D_{i}=\sum_{k} D_{i}^{k} X_{k}$ is the above decomposition of $D_{i}$ in $\mathcal{A}_{n}$.

Proposition 1.16 The map $\operatorname{div}: \mathfrak{t a}_{n} \rightarrow\left\langle\mathcal{A}_{n}\right\rangle$ is a 1-cocycle, that is

$$
\operatorname{div}[D, E]=D \cdot \operatorname{div} E-E \cdot \operatorname{div} D
$$

for all $D, E \in \mathfrak{t a d}_{n}$.

Proof. We first compute the $k$-th component of

$$
[D, E]_{i}=\left[D_{i}, E_{i}\right]+D\left(E_{i}\right)-E\left(D_{i}\right)=\left(D\left(E_{i}\right)+D_{i} E_{i}\right)-\left(E\left(D_{i}\right)+E_{i} D_{i}\right) .
$$

\footnotetext{
${ }^{7}$ This map is denoted by $a \mapsto \operatorname{tr}^{\text {quad }} a$ in [8].
} 
Clearly $\left(D_{i} E_{i}\right)^{k}=D_{i} E_{i}^{k}$. Besides, $D$ being a tangential derivation,

$$
\begin{aligned}
D\left(E_{i}\right) & =\sum_{k} D\left(E_{i}^{k} X_{k}\right)=\sum_{k} D\left(E_{i}^{k}\right) X_{k}+E_{i}^{k}\left[X_{k}, D_{k}\right] \\
& =\sum_{k}\left(D\left(E_{i}^{k}\right)-E_{i}^{k} D_{k}\right) X_{k}+\sum_{k, l} E_{i}^{k} X_{k} D_{k}^{l} X_{l} \\
& =\sum_{k}\left(D\left(E_{i}^{k}\right)-E_{i}^{k} D_{k}+\sum_{l} E_{i}^{l} X_{l} D_{l}^{k}\right) X_{k} .
\end{aligned}
$$

This gives the component $D\left(E_{i}\right)^{k}$ hence $[D, E]_{i}^{k}$ and the above equations easily imply, modulo $\left[\mathcal{A}_{n}, \mathcal{A}_{n}\right]$,

$\sum_{i}[D, E]_{i}^{i} X_{i} \sim \sum_{i}\left(D\left(E_{i}^{i}\right)-E_{i}^{i} D_{i}+D_{i} E_{i}^{i}\right) X_{i}-\left(E\left(D_{i}^{i}\right)-D_{i}^{i} E_{i}+E_{i} D_{i}^{i}\right) X_{i}$

On the other hand

$$
\begin{aligned}
D \cdot \operatorname{div} E & =\sum_{i}\left\langle D\left(E_{i}^{i} X_{i}\right)\right\rangle=\sum_{i}\left\langle D\left(E_{i}^{i}\right) X_{i}+E_{i}^{i} X_{i} D_{i}-E_{i}^{i} D_{i} X_{i}\right\rangle \\
& =\sum_{i}\left\langle D\left(E_{i}^{i}\right) X_{i}+D_{i} E_{i}^{i} X_{i}-E_{i}^{i} D_{i} X_{i}\right\rangle,
\end{aligned}
$$

hence $D \cdot \operatorname{div} E-E \cdot \operatorname{div} D=\sum_{i}\left\langle[D, E]_{i}^{i} X_{i}\right\rangle=\operatorname{div}[D, E]$.

This cocycle property of div allows defining a representation of the Lie algebra $\mathfrak{t a}_{n}$ on the graded vector space $\mathbb{K} u \oplus\left\langle\mathcal{A}_{n}\right\rangle$, where $u$ is some generator of degree zero, according to

$$
D \cdot(\lambda u+\langle a\rangle)=\lambda \operatorname{div} D+D \cdot\langle a\rangle
$$

with $D \in \mathfrak{t a}_{n}, \lambda \in \mathbb{K},\langle a\rangle \in\left\langle\mathcal{A}_{n}\right\rangle$. Indeed $[D, E] \cdot(\lambda u+\langle a\rangle)=(D E-E D)$. $(\lambda u+\langle a\rangle)$ by Proposition 1.16. The group $T A_{n}$ then acts on $\mathbb{K} u \oplus\left\langle\mathcal{A}_{n}\right\rangle$ by

$$
(\exp D) \cdot(\lambda u+\langle a\rangle)=\sum_{k=0}^{\infty} \frac{1}{n !} D^{n} \cdot(\lambda u+\langle a\rangle) .
$$

In particular $(\exp D) \cdot u=u+\operatorname{div} D+\frac{1}{2} D \cdot \operatorname{div} D+\frac{1}{3 !} D^{2} \cdot \operatorname{div} D+\cdots$

We now define the «logarithm of Jacobian» map $\mathcal{J}: T A_{n} \rightarrow\left\langle\mathcal{A}_{n}\right\rangle$ by

$$
\mathcal{J}(g):=g \cdot u-u \in\left\langle\mathcal{A}_{n}\right\rangle
$$

for $g \in T A_{n}$. The following properties of $\mathcal{J}$ are clear from this definition: for $g, h \in T A_{n}$ and $D \in \mathfrak{t o}_{n}$,

$$
\begin{aligned}
\mathcal{J}(g h) & =\mathcal{J}(g)+g \cdot \mathcal{J}(h) \\
\mathcal{J}(\exp D) & =\frac{e^{D}-1}{D} \cdot \operatorname{div} D \\
\left.\partial_{t} \mathcal{J}(\exp t D)\right|_{t=0} & =\operatorname{div} D .
\end{aligned}
$$


Proposition 1.17 Let $\Phi \in T A_{n}$ be a tangential automorphism of $\mathfrak{l}_{n}$ such that

$$
\Phi\left(X_{1}+\cdots+X_{n}\right)=X_{1}+\cdots+X_{n} .
$$

Then $\mathcal{J}(\Phi)=0$.

Proof. We have $\Phi=\exp D$ with $D \in \mathfrak{t}_{n}$. We shall prove $\operatorname{div} D=0$ and conclude by (1.33). The derivation $D$ decomposes as $D=\sum_{1}^{\infty} D_{k}$ with $D_{k} \in \mathfrak{t a}_{n}$ homogeneous of degree $k$. Let $S:=X_{1}+\cdots+X_{n}$, homogeneous of degree 1. Since $(\exp D)(S)=S$ we have

$$
S+\sum_{k \geq 1} D_{k}(S)+\frac{1}{2 !} \sum_{k, l \geq 1} D_{k} D_{l}(S)+\cdots=S .
$$

Separating homogeneous components we successively obtain $D_{1}(S)=0$, $D_{2}(S)+\frac{1}{2} D_{1}^{2}(S)=0$ hence $D_{2}(S)=0, \ldots$ and $D_{k}(S)=0$ for all $k$ inductively. Thus $D(S)=0$ and the proposition follows from the next lemma.

Lemma 1.18 Assume $D \in \mathfrak{t a d}_{n}$ and $D\left(X_{1}+\cdots+X_{n}\right)=0$. Then $\operatorname{div} D=$ 0 .

Proof. Writing $D=\left(D_{1}, \ldots, D_{n}\right)$ with $D_{i} \in \mathfrak{l}_{n}$ we have $\sum_{i}\left[X_{i}, D_{i}\right]=$ $\sum_{i}\left(X_{i} D_{i}-D_{i} X_{i}\right)=0$ that is, with the decomposition $D_{i}=\sum_{k} D_{i}^{k} X_{k}$,

$$
\sum_{i, k} X_{i} D_{i}^{k} X_{k}=\sum_{k} D_{k} X_{k}
$$

Thus $D_{k}=\sum_{i} X_{i} D_{i}^{k}$ for $k=1, \ldots, n$. Applying the anti-involution $\tau$ we get $-D_{k}=\sum_{i} \tau\left(D_{i}^{k}\right)\left(-X_{i}\right)$, hence $D_{k}^{i}=\tau\left(D_{i}^{k}\right)$. Then

$$
2 \sum_{i} D_{i}^{i} X_{i}=(1+\tau)\left(\sum_{i} D_{i}^{i} X_{i}\right)+(1-\tau)\left(\sum_{i} D_{i}^{i} X_{i}\right) .
$$

In the right-hand side the first term is $\sum_{i}\left(D_{i}^{i} X_{i}-X_{i} \tau\left(D_{i}^{i}\right)\right)=\sum_{i}\left[D_{i}^{i}, X_{i}\right] \in$ $\left[\mathcal{A}_{n}, \mathcal{A}_{n}\right]$, and the second term belongs to $\mathcal{N}_{n}$. Thus $\operatorname{div} D=0$.

Combining Section 1.8.3 with Proposition 1.17 (for $n=3$ ) we have proved that the automorphism $\Phi \in T A_{3}$ defined by $(1.31) \mathcal{F}_{2,3}^{-1} \mathcal{F}_{1,23}^{-1}=\mathcal{F}_{1,2}^{-1} \mathcal{F}_{12,3}^{-1} \Phi$ satisfies $\mathcal{J}(\Phi)=0$. The cocycle property (1.32) of $\mathcal{J}$ implies

$$
\mathcal{J}\left(\mathcal{F}_{2,3}^{-1}\right)+\mathcal{F}_{2,3}^{-1} \cdot \mathcal{J}\left(\mathcal{F}_{1,23}^{-1}\right)=\mathcal{J}\left(\mathcal{F}_{1,2}^{-1}\right)+\mathcal{F}_{1,2}^{-1} \cdot \mathcal{J}\left(\mathcal{F}_{12,3}^{-1}\right) .
$$

Let $f(X, Y) \in \mathcal{A}_{2}$ be such that $\langle f(X, Y)\rangle:=\mathcal{J}\left(\mathcal{F}^{-1}\right)$. Then $\mathcal{J}\left(\mathcal{F}_{2,3}^{-1}\right)=$ $\langle f(Y, Z)\rangle, \mathcal{J}\left(\mathcal{F}_{1,23}^{-1}\right)=\langle f(X, Y+Z)\rangle, \mathcal{J}\left(\mathcal{F}_{12,3}^{-1}\right)=\langle f(X+Y, Z)\rangle$ in $\left\langle\mathcal{A}_{3}\right\rangle$ and the above relation becomes

$$
\langle f(Y, Z)\rangle+\mathcal{F}_{2,3}^{-1} \cdot\langle f(X, Y+Z)\rangle=\langle f(X, Y)\rangle+\mathcal{F}_{1,2}^{-1} \cdot\langle f(X+Y, Z)\rangle .
$$

Remembering that $\mathcal{F}$ was built so that $\mathcal{F}(V(X, Y))=X+Y$ we obtain

$$
\langle f(Y, Z)+f(X, V(Y, Z))\rangle=\langle f(X, Y)+f(V(X, Y), Z)\rangle .
$$




\subsubsection{A Cohomology Argument}

We now solve equation (1.35).

Proposition 1.19 An element $f(X, Y) \in \mathcal{A}_{2}$ satisfies

$$
\langle f(Y, Z)+f(X, V(Y, Z))\rangle=\langle f(X, Y)+f(V(X, Y), Z)\rangle
$$

in $\left\langle\mathcal{A}_{3}\right\rangle$ if and only if there exists an even formal series $g(X) \in \mathcal{A}_{1}$ such that

$$
\langle f(X, Y)\rangle=\langle g(X)+g(Y)-g(V(X, Y))\rangle .
$$

Sketch of proof. (i) The «if» part follows from the associativity of $V$. (ii) A simplified version of the converse is proved first, with $V(X, Y)$ replaced by $X+Y$. Letting $\delta: \mathcal{A}_{2} \rightarrow \mathcal{A}_{3}$ be defined by ${ }^{8}$

$$
\delta f(X, Y, Z):=f(Y, Z)+f(X, Y+Z)-f(X, Y)-f(X+Y, Z),
$$

we show that $\langle\delta f\rangle=0$ implies the existence of $g$ such that

$$
\langle f(X, Y)\rangle=\langle g(X)+g(Y)-g(X+Y)\rangle .
$$

Since the map $\delta$ preserves homogeneity, it suffices to prove this for $f$ homogeneous of degree $k \geq 1$. Our assumption implies

$$
\left.\partial_{t}\langle\delta f(X, Y, t Y)-\delta f(t X, X, Y)\rangle\right|_{t=0}=0,
$$

hence

$k\langle f(X, Y)\rangle=\left.\partial_{t}\langle f(t X, X+Y)-f(t X, X)+f(X+Y, t Y)-f(Y, t Y)\rangle\right|_{t=0}$.

But, looking modulo brackets at the first order terms with respect to $t$ in $f(t X, Y)$, it is easily seen that $\left.\partial_{t}\langle f(t X, Y)\rangle\right|_{t=0}=\alpha\left\langle X Y^{k-1}\right\rangle$ for some $\alpha \in$ $\mathbb{K}$. Repeated application of this remark leads to

$$
k\langle f(X, Y)\rangle=\left\langle\alpha X(X+Y)^{k-1}-\alpha X^{k}+\beta Y(X+Y)^{k-1}-\beta Y^{k}\right\rangle
$$

for some $\alpha, \beta \in \mathbb{K}$.

If $k$ is odd we observe that $\left\langle A B^{k-1}\right\rangle=0$ in $\left\langle\mathcal{A}_{2}\right\rangle$ for all $A, B \in \mathfrak{l}_{2}$. Indeed, computing modulo $\left[\mathcal{A}_{2}, \mathcal{A}_{2}\right]+\mathcal{N}_{2}$,

$$
\left\langle A B^{k-1}\right\rangle=\left\langle\tau\left(A B^{k-1}\right)\right\rangle=(-1)^{k}\left\langle B^{k-1} A\right\rangle=(-1)^{k}\left\langle A B^{k-1}\right\rangle .
$$

Thus (1.36) implies $\langle f\rangle=0$ for $k$ odd.

If $k=2$ (1.36) boils down to $2\langle f(X, Y)\rangle=(\alpha+\beta)\langle X Y\rangle$ (ignoring a bracket) whence our claim with $g(X)=-(\alpha+\beta) X^{2} / 4$.

${ }^{8}$ More generally, one can define a family of maps $\delta:\left\langle\mathcal{A}_{n}\right\rangle \rightarrow\left\langle\mathcal{A}_{n+1}\right\rangle$ with $\delta^{2}=0$ (see 2.3 in [7]). We are proving here the vanishing of the second cohomology group. 
If $k$ is even, $k \geq 4$, we copy out (1.36) into $\langle\delta f\rangle=0$, which leads to

$$
\begin{aligned}
& k\langle\delta f(X, Y, Z)\rangle= \\
= & (\beta-\alpha)\left\langle Y\left((X+Y+Z)^{k-1}-(X+Y)^{k-1}-(Y+Z)^{k-1}+Y^{k-1}\right)\right\rangle=0 .
\end{aligned}
$$

Looking at the terms of highest degree with respect to $Y$, such as $\left\langle Y^{k-2} X Z\right\rangle$, we can conclude from this that $\alpha=\beta$. Then (1.36) implies our claim with $g(X)=-\alpha X^{k} / k$.

(iii) The original equation (1.35), with $V(X, Y)$ instead of $X+Y$, can be reduced to the simplified one by expanding $f$ into its homogeneous components and observing that $V(X, Y)=X+Y+\cdots$ where $\cdots$ have degree $\geq 2$. For details we refer to Appendix A of the printed version of [7] or to the arXiv version of [8].

\subsubsection{An Expression of the Divergence}

Let us summarize what is known at this point. Given $F, G \in \mathfrak{l}_{2}$ we have constructed a family of tangential automorphisms $\mathcal{F}_{t} \in T A_{2}$, solution of the differential equation (1.29)

$$
\mathcal{F}_{t}^{-1} \partial_{t} \mathcal{F}_{t}=-D_{t}, \mathcal{F}_{0}=e
$$

where $D_{t} \in \mathfrak{t a}_{2}$ is the tangential derivation $D_{t}=\left(F_{t}, G_{t}\right)$ with $F_{t}(X, Y)=$ $t^{-1} F(t X, t Y), G_{t}(X, Y)=t^{-1} G(t X, t Y)$. Setting $\mathcal{F}=\mathcal{F}_{1}$ we have $\mathcal{F}_{t}=$ $\Lambda_{t} \mathcal{F} \Lambda_{t}^{-1}$ (Lemma 1.15).

Furthermore, if $(F, G)$ satisfies $(\mathrm{KV} 1)$, we have proved $\mathcal{F}(V(X, Y))=$ $X+Y(1.30)$ and $\mathcal{J}\left(\mathcal{F}^{-1}\right)=\langle g(X)+g(Y)-g(V(X, Y))\rangle$ for some even series $g \in \mathcal{A}_{1}$ (see (1.35) and Proposition 1.19). The cocycle property (1.32) implies $\mathcal{J}(\mathcal{F})=-\mathcal{F} \cdot \mathcal{J}\left(\mathcal{F}^{-1}\right)$ and we infer that, in $\left\langle\mathcal{A}_{2}\right\rangle$,

$$
\mathcal{J}(\mathcal{F})=-\langle g(X)+g(Y)-g(X+Y)\rangle .
$$

Indeed $\mathcal{F}(V(X, Y))=X+Y$ and $\mathcal{F}(X)=e^{\text {ad } A} X, \mathcal{F}(Y)=e^{\text {ad } B} Y$ for some $A, B \in \mathfrak{l}_{2}$ (see the construction of $\mathcal{F}_{t}$ in 1.8.1) hence $\langle\mathcal{F}(X)\rangle=\langle X\rangle$ and $\langle\mathcal{F}(Y)\rangle=\langle Y\rangle$ in $\left\langle\mathcal{A}_{2}\right\rangle$.

The scaling formula $\mathcal{F}_{t}=\Lambda_{t} \mathcal{F} \Lambda_{t}^{-1}$ implies $\mathcal{J}\left(\mathcal{F}_{t}\right)=\Lambda_{t} \cdot \mathcal{J}(\mathcal{F})$ since, writing $\mathcal{F}=\exp E$ with $E \in \mathfrak{t o}_{2}$, we have

$$
\mathcal{J}\left(\mathcal{F}_{t}\right)=\mathcal{J}\left(\exp \left(\Lambda_{t} E \Lambda_{t}^{-1}\right)\right)=\Lambda_{t} \frac{e^{E}-1}{E} \Lambda_{t}^{-1} \cdot \operatorname{div}\left(\Lambda_{t} E \Lambda_{t}^{-1}\right)
$$

in view of (1.33). Also $\operatorname{div}\left(\Lambda_{t} E \Lambda_{t}^{-1}\right)=\Lambda_{t} \cdot \operatorname{div} E$ is easily checked from the definitions, hence

$$
\mathcal{J}\left(\mathcal{F}_{t}\right)=\Lambda_{t} \frac{e^{E}-1}{E} \cdot \operatorname{div} E=\Lambda_{t} \cdot \mathcal{J}(\mathcal{F})
$$


as claimed. Thus (1.37) implies

$$
\mathcal{J}\left(\mathcal{F}_{t}\right)=-\langle g(t X)+g(t Y)-g(t(X+Y))\rangle .
$$

This leads to an expression of $\operatorname{div} D$ (with $D=D_{1}$ ) as we now explain. Setting $t=1+s$ the differential equation for $\mathcal{F}_{t}$ yields the expansion

$$
\mathcal{F}^{-1} \mathcal{F}_{t}=e+\left.s \mathcal{F}^{-1} \partial_{t} \mathcal{J}\left(\mathcal{F}_{t}\right)\right|_{t=1}+\cdots=e-s D+\cdots
$$

where $\cdots$ have degree $\geq 2$ with respect to $s$. The definition of $\mathcal{J}$ now gives

$$
\begin{aligned}
\mathcal{J}\left(\mathcal{F}^{-1} \mathcal{F}_{t}\right) & =(e-s D+\cdots) \cdot u-u=-s D \cdot u+\cdots \\
& =-s \operatorname{div} D+\cdots
\end{aligned}
$$

But $\mathcal{F} \cdot \mathcal{J}\left(\mathcal{F}^{-1} \mathcal{F}_{t}\right)=\mathcal{J}\left(\mathcal{F}_{t}\right)-\mathcal{J}(\mathcal{F})$ by (1.32) again therefore, using (1.38),

$$
\mathcal{F} \operatorname{div} D=\langle h(X)+h(Y)-h(X+Y)\rangle
$$

where $h \in \mathcal{A}_{1}$ is the (even) series defined by $h(X):=X g^{\prime}(X)$. Finally

$$
\operatorname{div} D=\langle h(X)+h(Y)-h(V(X, Y))\rangle
$$

if $(F, G)$ satisfies $(\mathrm{KV} 1)$,

\subsubsection{Conclusion}

To conclude the proof of Theorem 1.14 it only remains to show that (1.39) actually holds with $h(X)=\frac{1}{2}\left(\frac{X}{2} \operatorname{coth} \frac{X}{2}-1\right)$.

Let $D=(F, G) \in \mathfrak{t o}_{2}$ be any tangential derivation and let

$$
F(X, Y)=f(x) Y+O\left(Y^{2}\right), G(X, Y)=\gamma X+g(x) Y+O\left(Y^{2}\right)
$$

be the expansions up to first order terms with respect to $Y$, with $x=\operatorname{ad} X$ as usual, $f, g \in \mathbb{K}[[t]], \gamma \in \mathbb{K}$ and $O\left(Y^{2}\right)$ denoting terms of degree $\geq 2$ in $Y$. In accordance with the remark in 1.8.1 there is no $X$ term in $F$.

a. We first prove that

$$
\operatorname{div} D=\left\langle(f(0)-f(X)+g(X)) Y+O\left(Y^{2}\right)\right\rangle .
$$

Indeed $\operatorname{div} D=\left\langle F^{X} X+G^{Y} Y\right\rangle$ by the definition of div with $F=F^{X} X+$ $F^{Y} Y, G=G^{X} X+G^{Y} Y$ and $F^{X}, \ldots, G^{Y} \in \mathcal{A}_{2}$. Writing $x^{k+1} Y=\left[X, x^{k} Y\right]=$ $-\left(x^{k} Y\right) X+X\left(x^{k} Y\right)$ we get $\left(x^{k} Y\right)^{Y}=X^{k}$ for all $k \geq 0$ by induction, hence $G^{Y} Y=g(X) Y+O\left(Y^{2}\right)$. Then

$$
\begin{aligned}
F^{X} X & =F-F^{Y} Y=f(x) Y-f(X) Y+O\left(Y^{2}\right) \\
& =(f(0)-f(X)) Y+O\left(Y^{2}\right)+\text { brackets }
\end{aligned}
$$


(since all terms in $f(x) Y$ except the first are Lie brackets) and (1.41) is proved.

b. Moreover, for any even series $h \in \mathcal{A}_{1}$, the right-hand side of (1.39) is

$$
\langle h(X)+h(Y)-h(V(X, Y))\rangle=\left\langle h(0)-h^{\prime}(X) Y+O\left(Y^{2}\right)\right\rangle .
$$

Indeed $h(Y)=h(0)+O\left(Y^{2}\right)$ since $h$ is even. Also $V=X+\varphi(x) Y+O\left(Y^{2}\right)$ with $\varphi(x):=x /\left(1-e^{-x}\right)$ by Lemma 1.2 , hence $V^{k}-X^{k}=k X^{k-1} \varphi(x) Y+$ $O\left(Y^{2}\right)+$ brackets and $h(V)-h(X)=h^{\prime}(X) \varphi(x) Y+O\left(Y^{2}\right)+$ brackets. But $\varphi(0)=1$ and $X^{k}\left(x^{l} Y\right)=\left[X, X^{k}\left(x^{l-1} Y\right)\right]$ for $l \geq 1$, so that the only remaining terms are $h(V)-h(X)=h^{\prime}(X) Y+O\left(Y^{2}\right)+$ brackets, which proves (1.42).

c. If $(F, G)$ satisfies (KV1) we then have, by (1.39), (1.41) and (1.42),

$$
\left\langle h(0)-h^{\prime}(X) Y+O\left(Y^{2}\right)\right\rangle=\left\langle(f(0)-f(X)+g(X)) Y+O\left(Y^{2}\right)\right\rangle .
$$

This implies

$$
h(0)=0, h^{\prime}(X)=f(X)-g(X)+e_{1}(X)
$$

where $e_{1} \in \mathcal{A}_{1}$ is some even series, as shown by the next lemma.

Lemma 1.20 Let $\lambda \in \mathbb{K}$ and $a \in \mathcal{A}_{1}$ be such that $\left\langle\lambda+a(X) Y+O\left(Y^{2}\right)\right\rangle=$ 0 . Then $\lambda=0$ and $a$ is even.

Proof. By assumption $\lambda+a(X) Y+O\left(Y^{2}\right)$ is the sum of Lie brackets and an element of $\mathcal{N}_{2}=\operatorname{ker}(1+\tau)$. Applying $1+\tau$ it follows that $2 \lambda+$ $(a(X)-a(-X)) Y+O\left(Y^{2}\right)$ is a sum of brackets. The zero order term with respect to $Y$ gives $\lambda=0$. Looking at first order terms it suffices to show that, if $f(X)=\sum_{k \geq 0} f_{k} X^{k}$ and $f(X) Y$ is a sum of brackets, then $f=0$. Assuming this and separating orders with respect to $X$, for every $k$ there exist scalars $c_{p q r} \in \mathbb{K}$ such that

$$
f_{k} X^{k} Y=\sum c_{p q r}\left[X^{p}, X^{q} Y X^{r}\right]=\sum c_{p q r}\left(X^{p+q} Y X^{r}-X^{q} Y X^{p+r}\right)
$$

where $\sum$ runs over $p \geq 1, q, r \geq 0$ and $p+q+r=k$. Each coefficient appears twice in the latter sum, with opposite signs; if we reorder it as

$$
f_{k} X^{k} Y=c_{0} X^{k} Y+c_{1} X^{k-1} Y X+\cdots+c_{k} Y X^{k},
$$

we have $c_{0}+\cdots+c_{k}=0$ of course. But the uniqueness of the decomposition (1.28) in $\mathcal{A}_{2}$ implies $c_{0}=f_{k}$ and $c_{1}=0, \ldots, c_{k}=0$ successively. Therefore $f_{k}=0$ and the lemma is proved.

d. The final step is to prove that $f, g$ in the expansions of $F$ and $G$ are determined by (KV1). Indeed, replacing $Y$ by $t Y$, we have $V(t Y, X)=$ 
$X+t \varphi(-x) Y+O\left(t^{2}\right)$ by Lemma 1.2 and the first order terms with respect to $t$ in (KV1) give, in view of (1.40),

$$
\varphi(-x) Y=Y-\left(1-e^{-x}\right) f(x) Y+\gamma x Y,
$$

hence $f(x)=(1-\varphi(-x)) /\left(1-e^{-x}\right)+\gamma \varphi(x)$ that is

$$
f(x)=\varphi^{\prime}(x)+\gamma \varphi(x) .
$$

To reach $g$ we need the second order terms in the expansion of (KV1). Thus let

$$
F(X, Y)=f(x) Y+\frac{1}{2}\left(a\left(x_{1}, x_{2}\right) \mid Y, Y\right)+O\left(Y^{3}\right)
$$

be the expansion of $F$, where $a(u, v)=\sum_{p, q \geq 0} a_{p q} u^{p} v^{q}$ is some formal (commutative) power series and we have written, for any $A, B \in \mathfrak{l}_{2}$,

$$
\left(a\left(x_{1}, x_{2}\right) \mid A, B\right):=\sum_{p, q} a_{p q}\left[x^{p} A, x^{q} B\right] .
$$

Since $x^{p} y x^{q} Y=(\operatorname{ad} X)^{p}\left[Y, x^{q} Y\right]=\left(\left(x_{1}+x_{2}\right)^{p} x_{2}^{q} \mid Y, Y\right)$ by Leibniz' formula (with $y=\operatorname{ad} Y$ ), the second order terms in $F \in \mathfrak{l}_{2}$ actually have the claimed form. Similarly, for $V(t):=V(t Y, X)$ we have $V^{\prime}(t)=\varphi(-\operatorname{ad} V(t)) Y$ by Lemma 1.2, noting that $e^{\operatorname{ad} V(t)} e^{-x} Y=e^{t y} Y=Y$. Let $\varphi(-x)=\sum_{p \geq 0} \varphi_{p} x^{p}$; then

$$
\begin{aligned}
V^{\prime}(t) & =\varphi\left(-x-t \operatorname{ad}(\varphi(-x) Y)+O\left(t^{2}\right)\right) Y \\
& =\varphi(-x) Y+t \sum_{p \geq 1} \varphi_{p} \sum_{1 \leq q \leq p} x^{q-1} \operatorname{ad}(\varphi(-x) Y) x^{p-q} Y+O\left(t^{2}\right) .
\end{aligned}
$$

But

$$
\begin{aligned}
x^{q-1} \operatorname{ad}(\varphi(-x) Y) x^{p-q} Y & =x^{q-1}\left[\varphi(-x) Y, x^{p-q} Y\right] \\
& =x^{q-1}\left(\varphi\left(-x_{1}\right) x_{2}^{p-q} \mid Y, Y\right) \\
& =\left(\left(x_{1}+x_{2}\right)^{q-1} \varphi\left(-x_{1}\right) x_{2}^{p-q} \mid Y, Y\right)
\end{aligned}
$$

and the sum for $1 \leq q \leq p$ gives $\left(\varphi\left(-x_{1}\right)\left(\left(x_{1}+x_{2}\right)^{p}-x_{2}^{p}\right) / x_{1} \mid Y, Y\right)$. It follows that $V^{\prime}(t)=\varphi(-x) Y+t\left(b\left(x_{1}, x_{2}\right) \mid Y, Y\right)+O\left(t^{2}\right)$ with

$$
b\left(x_{1}, x_{2}\right):=\varphi\left(-x_{1}\right) \frac{\varphi\left(-x_{1}-x_{2}\right)-\varphi\left(-x_{2}\right)}{x_{1}},
$$

hence

$$
V(t Y, X)=X+t \varphi(-x) Y+\frac{t^{2}}{2}\left(b\left(x_{1}, x_{2}\right) \mid Y, Y\right)+O\left(t^{3}\right) .
$$

Besides we have $\left(a\left(x_{1}, x_{2}\right) \mid Y, Y\right)=0$ if and only if $a$ is a symmetric series: $a(u, v)=a(v, u)$; this fact may be proved by means of a Hall basis of $\mathfrak{l}_{2}$ (see 
Proposition 2.6 in the arXiv version of [6]; cf. Lemma 4.34 below). Thus identification of the second order terms in (KV1) leads to

$$
b(u, v)=\left(e^{-u-v}-1\right) a(u, v)-2 g(v)+\gamma v+c(u, v)
$$

where $c$ is a symmetric series. Remembering the definition of $b$, the special case $v=X, u=-X$ gives

$$
2 g(X)=\varphi^{\prime}(X)+\gamma X+e_{2}(X)
$$

where $e_{2}(X)=c(-X, X)$ is an even series.

e. Let us gather all pieces. Since $\varphi(X)-(X / 2)=(X / 2) \operatorname{coth}(X / 2)$ is even we obtain $f-g=\frac{1}{2} \varphi^{\prime}+e$ with $e$ even and, $h$ being even, (1.43) may be rewritten as

$$
h^{\prime}=\frac{1}{2}\left(\varphi^{\prime}-\frac{1}{2}\right), h(0)=0
$$

hence $h(X)=\frac{1}{2}\left(\frac{X}{2} \operatorname{coth} \frac{X}{2}-1\right)$. With (1.39) this completes the proof of Theorem 1.14

\subsection{The Case of Quadratic or Solvable Lie Al- gebras}

We shall now explain how, for two important classes of Lie algebras, (KV2) can be deduced from (KV1) thanks to Theorem 1.14. This will prove the Kashiwara-Vergne conjecture in those cases.

Working first with $\mathfrak{l}_{2}$ as before, let $\mathcal{B}$ denote the algebra of all (associative but non-commutative) formal series in $x=\operatorname{ad} X$ and $y=\operatorname{ad} Y$. The adjoint representation ad $: \mathfrak{l}_{2} \rightarrow \operatorname{End}\left(\mathfrak{l}_{2}\right)$ extends to a morphism of associative algebras ad : $\mathcal{A}_{2} \rightarrow \mathcal{B} \subset \operatorname{End}\left(\mathfrak{l}_{2}\right)$; for example ad 1 is the identity, $\operatorname{ad}(X Y X) Z=$ $x y x Z=[X,[Y,[X, Z]]]$ for any $Z \in \mathfrak{l}_{2}$ etc. We still denote by $\tau$ the antiinvolution of $\mathcal{B}$ defined by $\tau(x)=-x, \tau(y)=-y$; thus $\tau(\operatorname{ad} a)=\operatorname{ad} \tau(a)$ for any $a \in \mathcal{A}_{2}$.

To define the partial differentials $\partial_{X} F, \partial_{Y} F$ of a Lie series $F \in \mathfrak{l}_{2}$, let $\mathfrak{l}_{3}$ be the free Lie algebra with generators $X, Y, Z$ and

$$
\partial_{X} F(X, Y) Z=\left.\partial_{t} F(X+t Z, Y)\right|_{t=0}, \partial_{Y} F(X, Y) Z=\left.\partial_{t} F(X, Y+t Z)\right|_{t=0} .
$$

These partial differentials are related to $F^{X}$ and $F^{Y}$ by the following lemma.

Lemma 1.21 Let $F \in \mathfrak{l}_{2}$ be a Lie series in $(X, Y)$ and $F=F^{X} X+F^{Y} Y$ its decomposition (1.28) in the algebra $\mathcal{A}_{2}$. Then

$$
\text { ad } F^{X}=\partial_{X} F(X, Y), \operatorname{ad} F^{Y}=\partial_{Y} F(X, Y),
$$

as endomorphisms of $\mathfrak{l}_{3}$. 
Proof. It suffices to prove the lemma for a Lie monomial of order $p \geq 1$, namely $F=\left[X_{1},\left[X_{2},\left[\ldots,\left[X_{p-1}, X_{p}\right] \ldots\right]\right.\right.$ with $X_{i}=X$ or $Y$ for all $i$. We use induction on $p$.

For $p=1$ we have (for instance) $F=X$ hence $F^{X}=1$ and $\partial_{X} F$ is the identity, $F^{Y}=0$ and $\partial_{Y} F=0$.

For $p \geq 2$ we have (for instance) $F=[X, G]$ where $G$ is some Lie monomial of order $p-1$ and we assume that ad $G^{X}=\partial_{X} G, \operatorname{ad} G^{Y}=\partial_{Y} G$. Then

$$
F(X+t Z, Y)=[X, G(X+t Z, Y)]+t[Z, G(X+t Z, Y)]
$$

and the derivatives at $t=0$ are

$$
\begin{aligned}
\partial_{X} F(X, Y) Z & =\left[X, \partial_{X} G(X, Y) Z\right]+[Z, G(X, Y)] \\
& =\left(\operatorname{ad} X \operatorname{ad} G^{X}-\operatorname{ad} G\right) Z=\operatorname{ad}\left(X G^{X}-G\right) Z
\end{aligned}
$$

by the inductive hypothesis. But $F=X G-G X=X\left(G^{X} X+G^{Y} Y\right)-G X$ gives $F^{X}=X G^{X}-G$, thus $\partial_{X} F=\operatorname{ad} F^{X}$. A similar proof for $\partial_{Y} F$ implies the lemma.

Corollary 1.22 (i) Let $F, G \in \mathfrak{l}_{2}$ be two Lie series in $(X, Y)$ such that (KV1) holds. Then there exists $b \in \mathcal{B}$ such that $\tau(b)=-b$ and

$$
x \circ \partial_{X} F+y \circ \partial_{Y} G \sim \frac{1}{2}\left(\frac{x}{2} \operatorname{coth} \frac{x}{2}+\frac{y}{2} \operatorname{coth} \frac{y}{2}-\frac{v}{2} \operatorname{coth} \frac{v}{2}-1\right)+b
$$

where $v=\operatorname{ad} V(X, Y)$ and $\sim$ means equality of formal series modulo $[\mathcal{B}, \mathcal{B}]$. (ii) Let $F, G$ be two Lie series on a finite-dimensional real Lie algebra $\mathfrak{g}$, solutions of (KV1) and analytic in a neighborhood of the origin in $\mathfrak{g} \times \mathfrak{g}$. Then there exists a series $\left(b_{k}\right)$ of (non-commutative) homogeneous polynomials of degree $k$ in $(x, y)$ such that $\tau\left(b_{k}\right)=-b_{k}$, the series $\sum_{k} \operatorname{tr}_{\mathfrak{g}} b_{k}$ converges and

$$
\begin{aligned}
\operatorname{tr}_{\mathfrak{g}}\left(x \circ \partial_{X} F\right. & \left.+y \circ \partial_{Y} G\right)= \\
& =\frac{1}{2} \operatorname{tr}_{\mathfrak{g}}\left(\frac{x}{2} \operatorname{coth} \frac{x}{2}+\frac{y}{2} \operatorname{coth} \frac{y}{2}-\frac{v}{2} \operatorname{coth} \frac{v}{2}-1\right)+\sum_{k \geq 0} \operatorname{tr}_{\mathfrak{g}} b_{k} .
\end{aligned}
$$

Proof. (i) Lemma 1.21 implies ad $\left(F^{X} X+G^{Y} Y\right)=\partial_{X} F \circ x+\partial_{Y} G \circ y$ and the result follows from Theorem 1.14 by the adjoint representation, with $b=\operatorname{ad} a$. Indeed $\tau(b)=\tau(\operatorname{ad} a)=\operatorname{ad} \tau(a)=-\operatorname{ad} a=-b$.

(ii) Let $u:=x \circ \partial_{X} F+y \circ \partial_{Y} G-\frac{1}{2}\left(\frac{x}{2} \operatorname{coth} \frac{x}{2}+\frac{y}{2} \operatorname{coth} \frac{y}{2}-\frac{v}{2} \operatorname{coth} \frac{v}{2}-1\right) \in \mathcal{B}$. From (i) we know that $u=b+c$ where $\tau(b)=-b$ and $c$ is a sum of brackets. Separating the homogeneous components $u_{k}, b_{k}, c_{k}$ of degree $k$ in $(x, y)$ we obtain $u_{k}=b_{k}+c_{k}$ where $\tau\left(b_{k}\right)=-b_{k}$ and $c_{k}$ is a finite sum of brackets. Under the assumptions of (ii), the series $u=\sum_{k \geq 0} u_{k}=\sum_{k \geq 0}\left(b_{k}+c_{k}\right)$ converges absolutely with respect to an operator norm and

$$
\operatorname{tr} u=\sum_{k \geq 0} \operatorname{tr} u_{k}=\sum_{k \geq 0} \operatorname{tr} b_{k} .
$$


Let us recall that, by definition, $\mathfrak{g}$ is quadratic if it has a $\mathfrak{g}$-invariant nondegenerate symmetric bilinear form. Letting $B$ denote this form, the invariance means that $B([X, Y], Z)+B(Y,[X, Z])=0$ for al $X, Y, Z$. A semisimple Lie algebra is quadratic, with its Killing form.

Theorem 1.23 Let $\mathfrak{g}$ be a finite-dimensional quadratic real Lie algebra and let $F, G \in \mathfrak{l}_{2}$ be two Lie series in $X, Y \in \mathfrak{g}$, solutions of (KV1) and analytic in a neighborhood of the origin in $\mathfrak{g} \times \mathfrak{g}$. Then they are solutions of (KV2) too.

Thus any solution of (KV1) (easily obtained as noted in 1.5.2.a) satisfies (KV2): the Kashiwara-Vergne problem is «trivial» in this case.

Proof. In view of Corollary 1.22 (ii) it suffices to check that $\operatorname{tr} b_{k}=0$ for all $k$. Here $x=\operatorname{ad} X$ and $y=\operatorname{ad} Y$ are skew-symmetric with respect to the invariant form, that is ${ }^{t} x=-x$ and ${ }^{t} y=-y$ where ${ }^{t}$ means transpose. Therefore ${ }^{t} b_{k}=\tau\left(b_{k}\right)=-b_{k}$ and $\operatorname{tr} b_{k}=0$.

The solvable case uses similar ideas but requires slightly more work.

Theorem 1.24 Let $\mathfrak{g}$ be a finite-dimensional solvable real Lie algebra and let $F, G \in \mathfrak{l}_{2}$ be two Lie series in $X, Y \in \mathfrak{g}$, solutions of (KV1) and analytic in a neighborhood of the origin in $\mathfrak{g} \times \mathfrak{g}$. Then the corresponding $\alpha$ - and $\beta$ invariant couple of Lie series (Proposition 1.12) is a solution of (KV1) and (KV2).

The Kashiwara-Vergne problem is «almost trivial» here: except for an elementary modification, any solution of (KV1) solves (KV2). The proof is based on Corollary 1.22 and the following lemma.

Lemma 1.25 (i) The relations $P:=\left(1-e^{-x}\right) F, Q:=\left(e^{y}-1\right) G$ imply

$$
x \circ \partial_{X} F+y \circ \partial_{Y} G \sim \frac{x}{1-e^{-x}} \partial_{X} P+\frac{y}{e^{y}-1} \partial_{Y} Q+\operatorname{ad}(F+G)
$$

where $\sim$ means equal modulo $[\mathcal{B}, \mathcal{B}]$.

(ii) Let $E:=P-Q$. If $(F, G)$ satisfy $(K V 1)$ then, modulo $[\mathcal{B}, \mathcal{B}]$,

$$
\begin{aligned}
& \left(x \circ \partial_{X} F+y \circ \partial_{Y} G\right)-\frac{1}{2}\left(\frac{x}{2} \operatorname{coth} \frac{x}{2}+\frac{y}{2} \operatorname{coth} \frac{y}{2}-\frac{v}{2} \operatorname{coth} \frac{v}{2}-1\right) \sim \\
& \sim \frac{1}{2}\left(\frac{x}{2} \operatorname{coth} \frac{x}{2} \circ \partial_{X} E-\frac{y}{2} \operatorname{coth} \frac{y}{2} \circ \partial_{Y} E\right)-\frac{1}{2}\left(\frac{v}{2} \operatorname{coth} \frac{v}{2}-1\right)+ \\
& + \text { ad }\left(F+G+\frac{X-Y}{4}\right) \text {. }
\end{aligned}
$$


Proof. (i) Let $\varphi(x):=x /\left(1-e^{-x}\right)$. Then $[X, F]=\varphi(x) P$ hence $x \partial_{X} F-$ ad $F=\partial_{X}(\varphi(x) P)$. Letting $P^{\prime}:=\varphi(x)^{-1} F$ we also have $\left[X, P^{\prime}\right]=P$ and $x \partial_{X} P^{\prime}-\operatorname{ad} P^{\prime}=\partial_{X} P$. It is then readily checked that, for any $n \geq 1$,

$$
\begin{aligned}
\partial_{X}\left(x^{n} P\right) & =\partial_{X}\left(x^{n+1} P^{\prime}\right)=x^{n+1} \partial_{X} P^{\prime}-x^{n} \text { ad } P^{\prime}-\sum_{k=1}^{n} x^{n-k} \text { ad }\left(x^{k} P^{\prime}\right) \\
& =x^{n} \partial_{X} P-\sum_{k=1}^{n}\left[x, x^{n-k} \operatorname{ad}\left(x^{k-1} P^{\prime}\right)\right] \sim x^{n} \partial_{X} P,
\end{aligned}
$$

hence $\partial_{X}(\varphi(x) P) \sim \varphi(x) \partial_{X} P$ and $x \partial_{X} F-\operatorname{ad} F \sim \varphi(x) \partial_{X} P$. Replacing $X, F, P$ by $Y, G, Q$ we obtain (i).

(ii) Let $V=V(X, Y), \bar{V}=V(Y, X)$ for short. In view of $P-Q=E$ and (KV1) written as $P+Q=X+Y-\bar{V}$, the first two terms in the right-hand side of (i) are

$$
\begin{aligned}
\varphi(x) \partial_{X} P+\varphi & (-y) \partial_{Y} Q= \\
& =\frac{1}{2} \varphi(x)\left(\partial_{X} E+1-\partial_{X} \bar{V}\right)-\frac{1}{2} \varphi(-y)\left(\partial_{Y} E-1+\partial_{Y} \bar{V}\right) .
\end{aligned}
$$

By Lemma $1.2 \partial_{X} \bar{V}=\varphi(\bar{v}) \varphi(x)^{-1}$ and $\partial_{Y} \bar{V}=\varphi(-\bar{v}) \varphi(-y)^{-1}$ with $\bar{v}=$ ad $\bar{V}$. But $\bar{v}=e^{-x} v e^{x}$ hence $\varphi(\bar{v})=e^{-x} \varphi(v) e^{x}$ and, modulo $[\mathcal{B}, \mathcal{B}]$,

$$
\varphi(x) \partial_{X} \bar{V} \sim \varphi(\bar{v}) \sim \varphi(v), \varphi(-y) \partial_{Y} \bar{V} \sim \varphi(-\bar{v}) \sim \varphi(-v)
$$

thus, using $\varphi(v)+\varphi(-v)=v \operatorname{coth} \frac{v}{2}$,

$\varphi(x) \partial_{X} P+\varphi(-y) \partial_{Y} Q \sim \frac{1}{2} \varphi(x)\left(\partial_{X} E+1\right)-\frac{1}{2} \varphi(-y)\left(\partial_{Y} E-1\right)-\frac{v}{2} \operatorname{coth} \frac{v}{2}$.

In the right-hand side we replace $\varphi$ by coth functions and observe that, by Lemma 1.7,

$$
x \partial_{X} E+y \partial_{Y} E \sim \partial_{X} E \circ x+\partial_{Y} E \circ y=\operatorname{ad} E \sim 0
$$

since $P, Q$ and $E$ belong to $\left[\mathcal{A}_{2}, \mathcal{A}_{2}\right]$ by their very definition. Then (ii) follows from (i).

Proof of Theorem 1.24. (i) Comparing Corollary 1.22 (i) and Lemma 1.25 (ii) we obtain

$$
\begin{aligned}
b \sim \frac{1}{2}\left(\frac{x}{2} \operatorname{coth} \frac{x}{2} \circ \partial_{X} E-\frac{y}{2} \operatorname{coth} \frac{y}{2} \circ \partial_{Y} E\right)- & \frac{1}{2}\left(\frac{v}{2} \operatorname{coth} \frac{v}{2}-1\right)+ \\
& +\operatorname{ad}\left(F+G+\frac{X-Y}{4}\right)
\end{aligned}
$$

modulo $[\mathcal{B}, \mathcal{B}]$. Given a solution $(F, G)$ of $(\mathrm{KV} 1)$ we may change it by Proposition 1.12 into an invariant solution under the symmetries $\alpha$ and $\beta$. Proposition $1.13(i)$ then shows that $E$ is odd and ad $\left(F+G+\frac{X-Y}{4}\right)$ is a sum 
of brackets. Besides $V^{\vee}:=V(-X,-Y)=-e^{-x} V$ (see (1.24)), therefore $v^{\vee}=\operatorname{ad} V^{\vee}=-e^{-x} v e^{x}$ and $(v / 2) \operatorname{coth}(v / 2)$ is even modulo brackets. We conclude that $b$ is even modulo $[\mathcal{B}, \mathcal{B}]$.

(ii) Let $x_{1} \cdots x_{n}$ be one of the monomials in $b$, with $x_{i}=x$ or $y$ for all $i$. The anti-involution $\tau$ gives

$$
\tau\left(x_{1} \cdots x_{n}\right)=(-1)^{n} x_{n} \cdots x_{1} \sim(-1)^{n} x_{1} \cdots x_{n}
$$

where $\sim$ now means an equality modulo the two-sided ideal $\mathcal{I}$ generated by $(x y-y x)$ in $\mathcal{B}$. Separating the even and odd components of $b$ we see that the property $\tau(b)=-b$ implies that $b$ is odd modulo $\mathcal{I}$. Since $[\mathcal{B}, \mathcal{B}] \subset \mathcal{I}$, we infer from (i) and (ii) that $b$ belongs to $\mathcal{I}$ and all its homogeneous components $b_{k}$ as well.

(iii) When $\mathfrak{g}$ is solvable, $x=\operatorname{ad} X$ and $y=\operatorname{ad} Y$ are given by upper triangular matrices in a suitable basis of its complexified Lie algebra (Lie's theorem). The matrix of $b_{k} \in \mathcal{I}$ is then strictly upper triangular, hence $\operatorname{tr} b_{k}=0$ and (KV2) follows by Corollary $1.22($ ii).

Remark. Lemma 1.25 leads to an equivalent form of the Kashiwara-Vergne conjecture: there exists a couple $(F, G)$ such that (KV1) and (KV2) if and only if there exists a couple $(F, G)$, invariant under the symmetries $\alpha$ and $\beta$, such that, setting $P=\left(1-e^{-x}\right) F, Q=\left(e^{y}-1\right) G, E=P-Q$ and $v=\operatorname{ad} V(X, Y)$, the following equations hold:

$$
\begin{gathered}
V(Y, X)=X+Y-P(X, Y)-Q(X, Y) \\
\operatorname{tr}_{\mathfrak{g}}\left(\frac{x}{2} \operatorname{coth} \frac{x}{2} \circ \partial_{X} E-\frac{y}{2} \operatorname{coth} \frac{y}{2} \circ \partial_{Y} E\right)=\operatorname{tr}_{\mathfrak{g}}\left(\frac{v}{2} \operatorname{coth} \frac{v}{2}-1\right) .
\end{gathered}
$$

The equivalence follows from Lemma 1.25 (ii), Propositions 1.12 and 1.13. This variant of the conjecture was used in the original article [30] $\$ 5$ for the solvable case.

\subsection{The General Case}

\subsubsection{An Abstract Formulation of the Kashiwara-Vergne Problem}

The formalism described in 1.8 allowed Alekseev and Torossian to give a complete proof of the Kashiwara-Vergne conjecture with the aid of Drinfeld's associators. We shall content ourselves here with a very brief survey of their method and refer to [7] and [2] for full details. Let us state and explain one of the main results of [7]. A few words about its proof are given in the next section.

Theorem 1.26 There exist $\mathcal{F} \in T A_{2}$ and $g \in \mathcal{A}_{1}$ such that (AT1) and 
(AT2) hold:

$$
\begin{gathered}
\mathcal{F}(V(X, Y))=X+Y \\
\mathcal{J}(\mathcal{F})=\langle g(X)+g(Y)-g(X+Y)\rangle .
\end{gathered}
$$

The group $T A_{2}$ of tangential automorphisms of the free Lie algebra $\mathfrak{l}_{2}$ was defined in 1.8.1, and we know from 1.8.2 that (KV1) for Lie series $F, G \in \mathfrak{l}_{2}$ implies (and is in fact equivalent to) the existence of $\mathcal{F} \in T A_{2}$ satisfying (AT1). Let us recall that $\mathcal{F}$ is obtained from $F$ and $G$ by integrating the tangential derivations $D_{t}=\left(F_{t}, G_{t}\right)$.

As in 1.8.4 we introduce the algebra $\mathcal{A}_{n}$ of associative but non-commutative formal series in $n$ generators. We denote here by $\left\langle\mathcal{A}_{n}\right\rangle=\mathcal{A}_{n}^{+} /\left[\mathcal{A}_{n}, \mathcal{A}_{n}\right]$ the vector space spanned by cyclic words in the generators and by $a \mapsto\langle a\rangle$ the canonical projection ${ }^{9}$. Note that, unlike the similar notation in 1.8.4, there is no $\tau$ and no $\mathcal{N}$ here. Except for this difference, the definitions and properties already given of div and $\mathcal{J}$ remain the same. As in 1.8.6 (AT1) and (AT2) imply the existence of a series $h \in \mathcal{A}_{1}$ (not necessarily even here) such that $\operatorname{div} D=\langle h(X)+h(Y)-h(V(X, Y))\rangle$. As in 1.8.7 it can be proved that $h(X)=\frac{1}{2}\left(\frac{X}{2} \operatorname{coth} \frac{X}{2}-1\right)+h_{-}(X)$ where $h_{-}$is odd. Finally the automorphism $\mathcal{F}$ can be modified into a new solution of (AT1) and (AT2) so as to obtain $h_{-}=0$, hence (KV2); see [7] $\S 5$ and $\S 6$.

Therefore Theorem 1.26 implies the Kashiwara-Vergne conjecture.

\subsubsection{Drinfeld's Associators and the Kashiwara-Vergne Conjecture}

Assuming (AT1) and (AT2) one can define $\Phi \in T A_{3}$ by $\Phi \mathcal{F}_{1,23} \mathcal{F}_{2,3}=$ $\mathcal{F}_{12,3} \mathcal{F}_{1,2}$ as in (1.31) and prove that $\Phi(X+Y+Z)=X+Y+Z$ as in 1.8.3. Besides $\mathcal{J}(\Phi)=0$ follows from the cocycle property of $\mathcal{J}$ together with (AT2) although Proposition 1.17 and Lemma 1.18, which made use of $\tau$ and $\mathcal{N}$, are no more valid here. One can also check that $\Phi$ satisfies the pentagon equation in $T A_{4}$

$$
\Phi_{12,3,4} \Phi_{1,2,34}=\Phi_{1,2,3} \Phi_{1,23,4} \Phi_{2,3,4}
$$

with a subscript notation similar to the one used in 1.8.3.

Remarkably, one can go backwards and prove Theorem 1.26 by letting $\Phi$ play the main role, as briefly sketched below. The following fundamental theorem is due to Drinfeld.

Theorem 1.27 There exists $\Phi \in T A_{3}$ such that

(i) $\Phi(X+Y+Z)=X+Y+Z$ and $\mathcal{J}(\Phi)=0$

(ii) $\Phi$ satisfies the pentagon equation, two hexagon equations and the inversion property $\Phi_{3,2,1}=\Phi_{1,2,3}^{-1}$

(iii) $\Phi$ belongs to the subgroup of $T A_{3}$ generated by infinitesimal braids.

\footnotetext{
${ }^{9}$ This map is denoted by $a \mapsto \operatorname{tr} a$ in [7].
} 
Such a $\Phi$ is called a Drinfeld associator. We refer to [7], [2] and the monograph [21] for detailed statements of (ii) and (iii). Drinfeld's proof exhibits an example of $\Phi$ arising from the monodromy of the Knizhnik-Zamolodchikov differential equation

$$
u^{\prime}(z)=\frac{1}{2 i \pi}\left(\frac{X}{z}+\frac{Y}{z-1}\right) u(z) .
$$

Given a Drinfeld associator $\Phi$, Alekseev and Torossian [7] construct an $\mathcal{F} \in T A_{2}$ such that $\Phi \mathcal{F}_{1,23} \mathcal{F}_{2,3}=\mathcal{F}_{12,3} \mathcal{F}_{1,2}$. The automorphism $\mathcal{F}$ is obtained as $\mathcal{F}=\exp f$, where the homogeneous components of $f \in \mathfrak{t a d}_{2}$ are determined inductively; a closed formula for $\mathcal{F}$ is given by Theorem 4 in [2]. From the property $\Phi(X+Y+Z)=X+Y+Z$ it follows that the Lie series $\chi(X, Y):=\mathcal{F}^{-1}(X+Y)$ satisfies the associativity $\chi(X, \chi(Y, Z))=$ $\chi(\chi(X, Y), Z)$. Looking at the expansion of $\chi$, the authors show that this implies $\chi(X, Y)=V(X, Y)$ hence (AT1).

Then, using the cocycle property of $j$ as we did at the end of 1.8.4, they prove that the property $\mathcal{J}(\Phi)=0$ implies (1.35) hence (AT2) by the cohomology argument of Proposition 1.19.

To sum up, from a Drinfeld associator one can thus construct a solution $\mathcal{F}$ of $(\mathrm{AT} 1)(\mathrm{AT} 2)$ hence a solution $(F, G)$ of the Kashiwara-Vergne conjecture. Moreover, the inversion property (ii) of $\Phi$ implies the invariance of this solution under the symmetry $\beta$ of 1.7.2.

\section{Notes}

In their pioneering 1978 paper [30] Masaki Kashiwara and Michèle Vergne proved that the convolution formula (1.1) for invariant distributions in a Lie group is implied by the «combinatorial» conjecture (KV1)(KV2) in its Lie algebra. They also proved the conjecture for all solvable Lie algebras.

Three years later I proved the conjecture in the note [42] for the simplest non-solvable algebra, $\operatorname{sl}(2, \mathbb{R})$, by means of ad hoc calculations which did not seem to extend beyond this case. Excepting this modest attempt, no significant progress occurred for nearly twenty years, until Vergne proved the conjecture for all quadratic Lie algebras in her 1999 note [59] by means of differential forms.

Things speeded up in the first decade of the twenty-first century. In 2001 Martin Andler, Siddhartha Sahi and Charles Torossian [9] proved the convolution property (1.1) (but not the combinatorial conjecture) by means of the Kontsevich star product [32]. In 2002, using the Moser trick and tools from Poisson geometry, Anton Alekseev and Eckhard Meinrenken [3] gave a new proof of the conjecture for quadratic Lie algebras. In 2006 [5], using the 2002 paper [54] by Torossian, the same authors gave the first complete proof of the conjecture. The Kontsevich deformation quantization was again one of the major tools in this approach; see Torossian's exposition [57]. Then, 
in 2008 Alekseev and Torossian [7] (see also [2]) gave a different proof of the conjecture, reducing the problem to the existence theorem of Drinfeld's associators. A recent paper by Carlo Rossi [41] sheds light on the link between both proofs.

In their 2009 note [8] Alekseev and Torossian showed that the KashiwaraVergne problem is «trivial» for quadratic Lie algebras and, following the ideas of [8] and [30], I noted a similar «triviality» for solvable algebras [48].

The reader is advised to browse through Torossian's web page ${ }^{10}$, which gathers many interesting documents.

Without any claim for giving here an exhaustive bibliography, let us also mention the papers [1], [6] and [12] related to the non-uniqueness problem in the conjecture.

Sections 1.2 to 1.5 are essentially taken from the fundamental 1978 paper [30]. The symmetries $\alpha$ and $\beta$ in 1.7 are used in [30] p. 265; the additional details on $\beta$ given here are from Section 8 of Alekseev and Torossian's article [7]. The expression (1.11) of $F$ and $G$ was given in my 1986 paper [43] and their relation to the original construction in [30] for the solvable case is explained in the appendix to [43]. Proposition 1.8 is mentioned without proof in [43] p. 562. Section 1.6 is taken from [42]; a simpler proof would be welcome.

Section 1.8 follows Alekseev and Torossian's works [7][8]. The proof of Theorem 1.14 given here is slightly different from the original however, after a suggestion given to me by Alekseev. The proof of Lemma 1.18 is due to Vergne. The study of second order terms in 1.8.7.d follows Emanuela Petracci's thesis (see [6]). Section 1.9 is taken from [8] for the quadratic case and [48] for the solvable case. Section 1.10 gives a very brief overview of the deep proof in [7] with Drinfeld's associators.

${ }^{10}$ http://www.math.jussieu.fr/ torossian/ 
46 CHAPTER 1. KASHIWARA-VERGNE METHOD FOR LIE GROUPS 


\section{Chapter 2}

\section{Convolution on Homogeneous Spaces}

In this short chapter we define convolution products on a general homogeneous space $G / H$. No symmetric space structure is assumed here. We give some examples, the most important being the application to invariant differential operators. Then we extend the definitions to line bundles over homogeneous spaces.

\subsection{Definition and Examples}

Let $G$ be a Lie group, $H$ a closed subgroup and $S=G / H$ the homogeneous space of all left cosets $g H, g \in G$. Let $\tau$ denote the action of $G$ on $G / H$, that is $\tau(g) g^{\prime} H=g g^{\prime} H$. A distribution $V$ on $S$ is $H$-invariant if $\langle V, f \circ \tau(h)\rangle=$ $\langle V, f\rangle$ for any test function $f \in \mathcal{D}(S)$ and any $h \in H$.

The convolution of a distribution $U$ by an $H$-invariant distribution $V$ is the distribution $U *_{S} V$ defined by

$$
\left\langle U *_{S} V, f\right\rangle:=\left\langle U(g H) \otimes V\left(g^{\prime} H\right), f\left(g g^{\prime} H\right)\right\rangle=\langle U(g H),\langle V, f \circ \tau(g)\rangle\rangle
$$

for any test function $f$; here $g, g^{\prime}$ denote variables in $G$. Due to the $H$ invariance of $V$ the term $\langle V, f \circ \tau(g)\rangle$ is a right $H$-invariant function of $g$, therefore defines a function of $g H$. The definition (2.1) makes sense under suitable assumptions on the supports of $U, V$ and $f$, e.g. if $V$ and $f$ are compactly supported. If $U$ and $V$ are $H$-invariant then $U *_{S} V$ is $H$-invariant too. If $V$ and $W$ are $H$-invariant we have $U *_{S}\left(V *_{S} W\right)=\left(U *_{S} V\right) *_{S} W$, as follows from the associativity of the multiplication in $G$.

If $S$ has a $G$-invariant measure $d x$ a (continuous) function $U$ on $S$ can be identified with the distribution $f \mapsto \int_{S} U(x) f(x) d x$. The convolution of two such distributions $U$ and $V$, the latter being $H$-invariant, is given by the 
integral

$$
\left(U *_{S} V\right)\left(g^{\prime} H\right)=\int_{S} U(g H) V\left(g^{-1} g^{\prime} H\right) d(g H) .
$$

Example 1: $H$ compact. The convolution $*_{S}$ on $S$ is then induced by the convolution $*_{G}$ on the group $G$.

Indeed the canonical projection $\pi: G \rightarrow G / H$ is here a proper map. Functions and distributions on $S$ can be pulled back to $G$ by

$$
\pi^{*} f=f \circ \pi,\left\langle\pi^{*} U, \varphi\right\rangle=\left\langle U, \pi_{*} \varphi\right\rangle \text { with } \pi_{*} \varphi(g H)=\int_{H} \varphi(g h) d h
$$

for $f \in \mathcal{D}(S), U \in \mathcal{D}^{\prime}(S)$ and $\varphi \in \mathcal{D}(G), d h$ being the Haar measure of $H$ normalized by $\int_{H} d h=1$. Note that $\pi_{*} \pi^{*} f=f$ and $\left\langle\pi^{*} U, \pi^{*} f\right\rangle=\langle U, f\rangle$. In particular, taking $\varphi(g)=\left\langle V\left(g^{\prime} H\right), f\left(g g^{\prime} H\right)\right\rangle$ for $H$-invariant $V$, resp. $\varphi\left(g^{\prime}\right)=$ $f\left(g g^{\prime} H\right)$, we simply have $\pi_{*} \varphi(g H)=\varphi(g)$ so that

$$
\begin{gathered}
\left\langle U(g H),\left\langle V\left(g^{\prime} H\right), f\left(g g^{\prime} H\right)\right\rangle\right\rangle=\left\langle\pi^{*} U(g),\left\langle V\left(g^{\prime} H\right), f\left(g g^{\prime} H\right)\right\rangle\right\rangle \\
=\left\langle\pi^{*} U(g),\left\langle\pi^{*} V\left(g^{\prime}\right), \pi^{*} f\left(g g^{\prime}\right)\right\rangle\right\rangle,
\end{gathered}
$$

that is

$$
\left\langle U *_{S} V, f\right\rangle=\left\langle\pi^{*} U *_{G} \pi^{*} V, \pi^{*} f\right\rangle .
$$

Here the right-hand side makes sense even if $V$ is not $H$-invariant. Then $\pi^{*}\left(U *_{S} V\right)=\pi^{*} U *_{G} \pi^{*} V$.

Example 2: group case. Let $\underline{G}$ be a Lie group, $G:=\underline{G} \times \underline{G}$ and let $H:=\{(g, g), g \in G\}$ be the diagonal subgroup. The map $\left(g_{1}, g_{2}\right) H \mapsto g_{1} g_{2}^{-1}$ identifies the homogeneous space $S=G / H$ with the group $\underline{G}$, an $H$-invariant distribution on $S$ with a central distribution on $G$ and the convolution (2.1) on $S$ with the convolution on $\underline{G}$ (which again makes sense even if $V$ is not $H$-invariant).

Indeed a function $f \in \mathcal{D}(S)$ corresponds with $\underline{f} \in \mathcal{D}(\underline{G})$ given by $f\left(\left(g_{1}, g_{2}\right) H\right)$ $=\underline{f}\left(g_{1} g_{2}^{-1}\right)$ i.e. $\underline{f}(g)=f((g, e) H)$, and a distribution $U \in \mathcal{D}^{\prime}(S)$ corresponds with $\underline{U} \in \mathcal{D}^{\prime}(\underline{G})$ given by $\langle U, f\rangle=\langle\underline{U}, f\rangle$ for all $f$. Now let $g_{1}, g_{2} \in \underline{G}$ be fixed. If $V$ is an $H$-invariant distribution on $S$ we may replace $\left(g_{1}^{\prime}, g_{2}^{\prime}\right)$ by $\left(g_{2}^{-1} g_{1}^{\prime}, g_{2}^{-1} g_{2}^{\prime}\right)$ so as to obtain:

$$
\left\langle V\left(\left(g_{1}^{\prime}, g_{2}^{\prime}\right) H\right), f\left(\left(g_{1}, g_{2}\right)\left(g_{1}^{\prime}, g_{2}^{\prime}\right) H\right)\right\rangle=\left\langle V\left(\left(g_{1}^{\prime}, g_{2}^{\prime}\right) H\right), f\left(\left(g_{1} g_{2}^{-1} g_{1}^{\prime}, g_{2}^{\prime}\right) H\right)\right\rangle .
$$

Introducing the function $\varphi\left(\left(g_{1}^{\prime}, g_{2}^{\prime}\right) H\right):=f\left(\left(g_{1} g_{2}^{-1} g_{1}^{\prime}, g_{2}^{\prime}\right) H\right)$ this expression equals

$$
\langle V, \varphi\rangle=\langle\underline{V}, \underline{\varphi}\rangle
$$

with

$$
\underline{\varphi}\left(g^{\prime}\right)=\varphi\left(\left(g^{\prime}, e\right) H\right)=f\left(\left(g_{1} g_{2}^{-1} g^{\prime}, e\right) H\right)=\underline{f}\left(g_{1} g_{2}^{-1} g^{\prime}\right) .
$$

Therefore

$$
\langle V, \varphi\rangle=\left\langle\underline{V}\left(g^{\prime}\right), \underline{f}\left(g_{1} g_{2}^{-1} g^{\prime}\right)\right\rangle=F\left(\left(g_{1}, g_{2}\right) H\right)
$$


with $\underline{F}(g):=\left\langle\underline{V}\left(g^{\prime}\right), f\left(g g^{\prime}\right)\right\rangle$, and finally

$$
\begin{aligned}
\left\langle U *_{S} V, f\right\rangle=\langle U, F\rangle & =\langle\underline{U}, \underline{F}\rangle=\left\langle\underline{U}(g),\left\langle\underline{V}\left(g^{\prime}\right), \underline{f}\left(g g^{\prime}\right)\right\rangle\right\rangle \\
& =\left\langle\underline{U} *_{\underline{G}} \underline{V}, \underline{f}\right\rangle
\end{aligned}
$$

as claimed.

Example 3: invariant differential operators. Let $\mathbb{D}(S)$ denote the algebra of linear differential operators $D$ on the homogeneous space $S=G / H$ which are $G$-invariant, that is $D(f \circ \tau(g))=(D f) \circ \tau(g)$ for any $f \in \mathcal{D}(S)$, $g \in G$. An element $D$ of $\mathbb{D}(S)$ acts on $\mathcal{D}(S)$ or $C^{\infty}(S)$ and, by transposition, on distributions:

$$
\left\langle{ }^{t} D U, f\right\rangle:=\langle U, D f\rangle, U \in \mathcal{D}^{\prime}(S), f \in \mathcal{D}(S) .
$$

The operator ${ }^{t} D$ preserves $H$-invariance.

If $V$ is $H$-invariant,

$$
\begin{aligned}
\left\langle{ }^{t} D\left(U *_{S} V\right), f\right\rangle & =\langle U(g H),\langle V,(D f) \circ \tau(g)\rangle\rangle \\
& =\left\langle U(g H),\langle V, D(f \circ \tau(g)\rangle\rangle=\left\langle U *{ }^{t} D V, f\right\rangle,\right.
\end{aligned}
$$

therefore

$$
{ }^{t} D\left(U *_{S} V\right)=U *_{S}{ }^{t} D V .
$$

In particular, $\delta$ denoting the Dirac measure at the origin $o=H$ of $S$, we have ${ }^{t} D \delta \in \mathcal{D}^{\prime}(S)^{H}$ and

$$
{ }^{t} D U=U *_{S}{ }^{t} D \delta
$$

Also ${ }^{t}\left(D \circ D^{\prime}\right) \delta={ }^{t} D \delta *{ }^{t} D^{\prime} \delta$ for $D, D^{\prime} \in \mathbb{D}(S)$.

Conversely, let $T$ be an element of $\mathcal{D}^{\prime}(S)^{H}$ supported at $o$. Defining $D$ by

$$
D f(g H):=\langle T, f \circ \tau(g)\rangle, f \in \mathcal{D}(S), g \in G,
$$

we get a linear map $D: \mathcal{D}(S) \rightarrow C^{\infty}(S)$ decreasing supports, therefore a differential operator by Peetre's theorem ([28] p. 236), clearly $G$-invariant on $S$. Thus $D \in \mathbb{D}(S)$ and ${ }^{t} D \delta=T$.

Remark. Assume $S=G / H$ has a $G$-invariant measure $d \mu$. Then $D \in \mathbb{D}(S)$ implies ${ }^{t} D \in \mathbb{D}(S)$. Indeed we have, for $f_{1}, f_{2} \in \mathcal{D}(S)$ and $g \in G$,

$$
\begin{aligned}
\int_{S}{ }^{t} D\left(f_{1} \circ \tau(g)\right) \cdot f_{2} d \mu & =\int\left(f_{1} \circ \tau(g)\right) \cdot D f_{2} d \mu \\
& =\int f_{1} \cdot\left(D f_{2} \circ \tau\left(g^{-1}\right)\right) d \mu \\
& =\int f_{1} \cdot D\left(f_{2} \circ \tau\left(g^{-1}\right)\right) d \mu \\
& =\int{ }^{t} D f_{1} \cdot\left(f_{2} \circ \tau\left(g^{-1}\right)\right) d \mu \\
& =\int_{S}\left({ }^{t} D f_{1} \circ \tau(g)\right) \cdot f_{2} d \mu
\end{aligned}
$$


in view of the invariance of $d \mu$ and $D$; thus ${ }^{t} D$ commutes with $\tau(g)$, as claimed.

\subsection{Extension to Line Bundles}

\subsubsection{The Line Bundle $L_{\chi}$}

Given a Lie group $G$, a closed subgroup $H$ and a character $\chi$ of $H$ (a morphism of $H$ into the multiplicative group of positive numbers) the corresponding line bundle above $G / H$ is

$$
L_{\chi}:=G \times_{H} \mathbb{C},
$$

the set of classes of couples $(x, z) \in G \times \mathbb{C}$ under the equivalence relation

$$
(x, z) \sim\left(x h, \chi(h)^{-1} z\right), h \in H .
$$

The class of $(x, z)$, denoted by $\underline{(x, z)} \in L_{\chi}$, projects in the base space as $x H \in G / H$.

If $\psi$ is a character of the whole group $G$, the map $(x, z) \mapsto\left(x, \psi(x)^{-1} z\right)$ defines an isomorphism of $L_{\chi}$ onto $L_{\chi \psi}$.

A smooth section $s: G / H \rightarrow L_{\chi}$ can be identified with a complex valued function $f \in C^{\infty}(G)$ such that

$$
f(x h)=\chi(h)^{-1} f(x)
$$

for all $x \in G, h \in H$, by the relation $s(x H)=\underline{(x, f(x))}$. Let $\Gamma\left(L_{\chi}\right)$ denote the space of smooth sections.

Example. If $d$ is a positive constant, the character

$$
\chi_{d}(h):=\left|\operatorname{det}_{\mathfrak{g} / \mathfrak{h}} \operatorname{Ad} h\right|^{-d}
$$

(the absolute value may be forgotten if $H$ is connected) defines a line bundle, the sections of which are the $d$-densities on $G / H$; for $d=0$ the 0 -densities are functions and the space of sections is $C^{\infty}(G / H)$. The special case $d=1 / 2$ will be important in the sequel.

The map $(x, z) \mapsto g \cdot(x, z)=(g x, z)$ defines a natural action of $g \in G$ on $L_{\chi}$, whence its action on a section $s$ :

$$
s_{g}(x H):=g^{-1} \cdot s(g x H) .
$$

Thus $s_{g g^{\prime}}=\left(s_{g}\right)_{g^{\prime}}$. If $s$ corresponds to a function $f$ on $G$, then $s_{g}$ corresponds to $f_{g}(x)=f(g x)$; a section is $H$-invariant if and only if the corresponding function is left $H$-invariant on $G$.

A linear differential operator $D: \Gamma\left(L_{\chi}\right) \rightarrow \Gamma\left(L_{\chi}\right)$ is $G$-invariant if $(D s)_{g}=$ $D\left(s_{g}\right)$ for all $s$ and $g$. Let $\mathbb{D}\left(L_{\chi}\right)$ denote the algebra of these operators under 
addition and composition. If $\psi$ is a character of the whole group $G$, the map $D \mapsto \psi^{-1} \circ D \circ \psi$ (where $\psi$ is the multiplication operator by $\psi(x)$ ) is an algebra isomorphism of $\mathbb{D}\left(L_{\chi}\right)$ onto $\mathbb{D}\left(L_{\chi \psi}\right)$. The algebra $\mathbb{D}\left(L_{\chi}\right)$ can be described in terms of enveloping algebras (see Notes below) but we shall not need it in the sequel.

\subsubsection{Convolution of Distributions on $L_{\chi}$}

A distribution $U$ on $L_{\chi}$ is a continuous linear form on the space of compactly supported smooth sections, equipped with the Schwartz topology. It is $H$ invariant if

$$
\left\langle U, s_{h}\right\rangle=\chi(h)^{-1}\langle U, s\rangle
$$

for all $h \in H$ and all compactly supported smooth sections $s$. For example the Dirac distribution $\delta$ defined by $\langle\delta, s\rangle=f(e)($ with $s(x H)=\underline{(x, f(x))})$ and, more generally, the distribution ${ }^{t} D \delta$ defined by

$$
\left\langle{ }^{t} D \delta, s\right\rangle=\langle\delta, D s\rangle
$$

are $H$-invariant for any $D \in \mathbb{D}\left(L_{\chi}\right)$.

The convolution of $U$ by an $H$-invariant distribution $V$ on $L_{\chi}$ (with suitable supports) is the distribution $U * V$ defined by

$$
\langle U * V, s\rangle=\langle U, \sigma\rangle \text { with } \sigma(x H)=\underline{\left(x,\left\langle V, s_{x}\right\rangle\right)} .
$$

The convolution of two $H$-invariant distributions is $H$-invariant. Moreover

$$
\begin{gathered}
{ }^{t} D(U * V)=U *{ }^{t} D V, U *{ }^{t} D \delta={ }^{t} D U \\
{ }^{t} D \delta *{ }^{t} D^{\prime} \delta={ }^{t}\left(D \circ D^{\prime}\right) \delta .
\end{gathered}
$$

if $D, D^{\prime} \in \mathbb{D}\left(L_{\chi}\right)$ and $V$ is $H$-invariant.

\section{Notes}

The algebra $\mathbb{D}\left(L_{\chi}\right)$ has been studied in various papers, in particular by Michel Duflo [19] for symmetric spaces, Tom Koornwinder [35] for general (non necessarily reductive) homogeneous spaces, Goro Shimura [50] for Hermitian symmetric spaces and Katsuhiro Minemura [38] for reductive homogeneous spaces. 


\section{Chapter 3}

\section{The Role of $e$-Functions}

Throughout this chapter $S=G / H$ denotes a ( $n$-dimensional) connected and simply connected symmetric space, where $G$ is a simply connected real Lie group (we may assume this) with involutive automorphism $\sigma$ and $H$ is the connected component of the identity in the fixed point subgroup of $\sigma$ in $G$. See Notation for more details; the main use of these topological assumptions is to specify the open sets we are working on. We still denote by $\sigma$ the corresponding automorphism of the Lie algebra $\mathfrak{g}$, whence the decomposition $\mathfrak{g}=\mathfrak{h} \oplus \mathfrak{s}$.

In Section 3.1 we define a transfer map $\sim$ from $\mathfrak{s}$ to $S$ for functions and distributions by means of the exponential map. We then introduce the concept of $e$-function, which appears when transferring convolutions back from $S$ to $\mathfrak{s}$. Section 3.2 is devoted to the simple case of special symmetric spaces, when $e$ is identically 1 and convolutions of $H$-invariant distributions in $\mathfrak{s}$ and $S$ correspond under $\sim$. Disregarding an existence proof of $e$-functions (postponed until Chapter 4), we develop in Section 3.3 applications of $e$ functions to invariant differential operators then, in Section 3.4, to mean value operators and spherical functions of Riemannian symmetric spaces. No specific construction of $e$ is required in those sections.

Section 3.5 explains how to obtain an $e$-function from certain integral formulas, if $H$ is a compact group. This is the method used in Section 3.7 to produce an explicit $e$-function for rank one Riemannian symmetric spaces of the noncompact type (the hyperbolic spaces). In the (partly conjectural) Section 3.6 we investigate some links between $e$-functions and spherical functions on a semisimple Lie group, arising from asymptotic properties as one goes to infinity in the positive Weyl chamber.

The chapter ends with an extension of $e$-functions to line bundles over a symmetric space (Section 3.8), and a list of open problems (Section 3.9).

The main features of this chapter are Definition 3.3 of $e$-functions, Proposition 3.5 (properties of special symmetric spaces), Theorems 3.8 and 3.9 (link with invariant differential operators), Theorem 3.13 and Corollary 3.14 (ex- 
pansion of mean value operators and spherical functions by means of $e$ ), Theorem 3.18 (another link between $e$ and spherical functions) and Theorem 3.23 (an explicit $e$-function for all hyperbolic spaces).

\subsection{Transferring Convolutions}

\subsubsection{The Transfer Map}

Our goal is to relate analysis of $H$-invariant functions (or distributions) on the symmetric space $S$ to analysis on its tangent space $\mathfrak{s}$ at the origin, by means of the exponential mapping Exp : $\mathfrak{s} \rightarrow S$ (normal coordinates).

First we need to restrict ourselves to an open set on which Exp is a diffeomorphism. For the group $G$ we recall from Section 1.3 that there exists a connected open neighborhood $\mathfrak{g}^{\prime}$ of 0 in $\mathfrak{g}$, invariant under $\operatorname{Ad} G, \sigma$ and all maps $X \mapsto t X, t \in[-1,1]$, such that $\exp : \mathfrak{g}^{\prime} \rightarrow \exp \mathfrak{g}^{\prime}$ is a diffeomorphism.

Lemma 3.1 The set $\mathfrak{s}^{\prime}:=\mathfrak{s} \cap \frac{1}{2} \mathfrak{g}^{\prime}$ is a connected open neighborhood of the origin in $\mathfrak{s}$, invariant under all maps $X \mapsto t X$ and $X \mapsto h \cdot X$ for $t \in[-1,1]$, $h \in H$, and such that $\operatorname{Exp}: \mathfrak{s}^{\prime} \rightarrow S^{\prime}:=\operatorname{Exp} \mathfrak{s}^{\prime}$ is a diffeomorphism.

If $G$ is simply connected we may take

$$
\mathfrak{s}^{\prime}=\{X \in \mathfrak{s}|| \operatorname{Im} \lambda \mid<\pi / 2, \text { for all eigenvalues } \lambda \text { of } \operatorname{ad} X \text { on } \mathfrak{g}\} .
$$

If Exp is a global diffeomorphism, e.g. if $S$ is a Riemannian symmetric space of the noncompact type ([27] p.253), we may replace $\mathfrak{s}^{\prime}$ by $\mathfrak{s}$.

Proof. Let $g H$ denote a coset in $\operatorname{Exp} \mathfrak{s}^{\prime}$; then $g=e^{X} h$ for some $X \in \mathfrak{s}^{\prime}$ and $h \in H$. By the symmetry $\sigma$ we get $\sigma g=e^{-X} h$ therefore $e^{2 X}=g(\sigma g)^{-1}$. But $2 X$ belongs to $\mathfrak{g}^{\prime}$ and, in view of the properties of $\mathfrak{g}^{\prime}$, this implies $X=$ $\frac{1}{2} \log \left(g(\sigma g)^{-1}\right) \in \mathfrak{s}^{\prime}$. It follows that Exp : $\mathfrak{s}^{\prime} \rightarrow \operatorname{Exp} \mathfrak{s}^{\prime}$ is a diffeomorphism.

Definition 3.2 Let $j$ be a given analytic function on $\mathfrak{s}^{\prime}$, strictly positive and $H$-invariant, normalized by $j(0)=1$. We transfer a function $f$ on $\mathfrak{s}^{\prime}$ into the function $\tilde{f}$ on $S^{\prime}$ defined by

$$
j(X) \tilde{f}(\operatorname{Exp} X)=f(X), X \in \mathfrak{s}^{\prime},
$$

and a distribution $u$ on $\mathfrak{s}^{\prime}$ into the distribution $\widetilde{u}$ on $S^{\prime}$ defined by

$$
\langle\widetilde{u}, \tilde{f}\rangle=\langle u, f\rangle
$$

for all $f \in \mathcal{D}\left(\mathfrak{s}^{\prime}\right)$. These definitions extend in an obvious way with $\mathfrak{s}^{\prime}$ replaced by an open subset of $\mathfrak{s}^{\prime}$. 
Remarks. (a) The transfer preserves $H$-invariance.

(b) For the time being there is no need for a specific choice of the factor $j$; we might for instance take $j=1$. It will soon become clear however that the most interesting choice is the square root of the Jacobian of Exp, i.e. $j(X)^{2}=$ $J(X)=\operatorname{det}_{\mathfrak{s}}(\operatorname{sh}(\operatorname{ad} X) / \operatorname{ad} X)$; see Example 1 below, or Propositions 3.6 and 4.23 , or the specific properties of the line bundle of half-densities (Sections 3.8 and 4.5). The particular choice $j=J^{1 / 2}$ will always be specified in the sequel.

Example 1: transferring measures. Let $d X$ be a Lebesgue measure on $\mathfrak{s}$ and let $a(X) d X$ be the distribution on $\mathfrak{s}^{\prime}$ defined by a locally integrable function $a$. The distribution $(a(X) d X)^{\sim}$ on $S^{\prime}$ is defined by

$$
\begin{aligned}
\langle\widetilde{a d X}, \widetilde{f}\rangle & =\langle a d X, f\rangle=\int_{\mathfrak{s}^{\prime}} a(X) f(X) d X \\
& =\int_{\mathfrak{s}^{\prime}} \widetilde{a}(\operatorname{Exp} X) \widetilde{f}(\operatorname{Exp} X) j(X)^{2} d X,
\end{aligned}
$$

for all $f \in \mathcal{D}\left(\mathfrak{s}^{\prime}\right)$. In other words

$$
(a(X) d X)^{\sim}=\widetilde{a}(x) d \mu(x),
$$

where $d \mu(x):=(j(X) d X)^{\sim}$ is the measure on $S^{\prime}$ given by

$$
\int_{S^{\prime}} \varphi(x) d \mu(x)=\int_{\mathfrak{s}^{\prime}} \varphi(\operatorname{Exp} X) j(X)^{2} d X, \varphi \in \mathcal{D}\left(S^{\prime}\right) .
$$

If $d X$ is $H$-invariant, $S$ has a $G$-invariant measure $d x$ (normalized so that $d \operatorname{Exp} X=J(X) d X)$ and it follows that $d \mu(x)=\left(j^{2} J^{-1}\right)(\log x) d x$ where Log is the inverse map of Exp. Thus $j=J^{1 / 2}$ is the only choice of $j$ such that the definitions of $\sim$ for functions and distributions agree through $(a(X) d X)^{\sim}=$ $\widetilde{a}(x) d x$.

Example 2: transferring invariant differential operators. We consider $\mathbb{D}(\mathfrak{s})^{H}$, the algebra of $H$-invariant (linear) differential operators on $\mathfrak{s}$ with constant coefficients, and $\mathbb{D}(S)$, the algebra of $G$-invariant (linear) differential operators on $S$. Let $\delta_{0}$, resp. $\delta$, denote the Dirac measure at the origin of $\mathfrak{s}$, resp. $S$; then $\widetilde{\delta_{0}}=\delta$. For $P \in \mathbb{D}(\mathfrak{s})^{H}$ the distribution $\left({ }^{t} P \delta_{0}\right)^{\sim}$ belongs to $\mathcal{D}^{\prime}(S)^{H}$ and is supported at the origin. As noted in Example 3 of 2.1 there exists a (unique) differential operator $\widetilde{P} \in \mathbb{D}(S)$ such that

$$
{ }^{t} \widetilde{P} \delta=\left({ }^{t} P \delta_{0}\right)^{\sim} .
$$

More generally, by (2.3),

$$
{ }^{t} \widetilde{P} U=U *_{S}\left({ }^{t} P \delta_{0}\right)^{\sim}
$$


for any distribution $U$ on $S$.

The definition of $\widetilde{P}$ means that $\widetilde{P} \widetilde{f}(o)=P f(0)$ for any smooth function $f$ on $\mathfrak{s}$, an equality only valid at the origin in general; the appropriate generalization will be given by Theorem 3.8. Taking $\varphi \in C^{\infty}(S), g \in G$ and $f(X):=j(X) \varphi(g \cdot \operatorname{Exp} X)$ we have $\widetilde{f}(x)=\varphi(g \cdot x)$ and the correspondence $P \mapsto \widetilde{P}$ is explicitly given by

$$
\widetilde{P} \varphi(g H)=\left.P(j(X) \varphi(g \cdot \operatorname{Exp} X))\right|_{X=0},
$$

$P$ acting on $X \in \mathfrak{s}$ in the right-hand side. Conversely, with $\log =\mathrm{Exp}^{-1}$ near the origin,

$$
P f(X)=\left.\widetilde{P}\left((j(\log x))^{-1} f(X+\log x)\right)\right|_{x=o}
$$

for $f \in C^{\infty}(\mathfrak{s}), X \in \mathfrak{s}$ and $\widetilde{P}$ acting on $x \in S$. The map $P \mapsto \widetilde{P}$ is an order preserving linear isomorphism of $\mathbb{D}(\mathfrak{s})^{H}$ onto $\mathbb{D}(S)$, but not in general an isomorphism of algebras; see Proposition 3.5 and Theorem 3.9 below.

For $j=1$ our map is the symmetrization map $\beta: P \mapsto \widetilde{P}=D_{\lambda(P)}$ considered by Helgason [28] p. 287; it should not be confused of course with the transfer of differential operators by means of the diffeomorphism Exp. For general $j$, (3.3) may be written as a Fischer product (see Section 3.3.1)

$$
(\widetilde{P} \varphi)(g H)=\langle P(\xi) \mid j(X) \varphi(g \cdot \operatorname{Exp} X)\rangle=\left\langle j\left(\partial_{\xi}\right) P(\xi) \mid \varphi(g \cdot \operatorname{Exp} X)\right\rangle,
$$

where $j\left(\partial_{\xi}\right)$ is an infinite order differential operator acting on the variable $\xi \in \mathfrak{s}^{*}$, in other $\operatorname{words}^{1} \widetilde{P}=\beta(j(\partial) P)$.

\subsection{2 e-Functions}

A function $j$ being chosen, we retain the notation $\sim$ from Definition 3.2 and now try to transfer convolutions. On $\mathfrak{s}$ the classical abelian convolution $*_{\mathfrak{s}}$ of two distributions $u, v$ is defined by

$$
\left\langle u *_{\mathfrak{s}} v, f\right\rangle=\langle u(X) \otimes v(Y), f(X+Y)\rangle .
$$

But a simple convolution formula like $\widetilde{u} *_{S} \widetilde{v}=\left(u *_{\mathfrak{s}} v\right)^{\sim}$ does not hold for general symmetric spaces: see Proposition 3.7 for instance. Instead convolutions of $H$-invariant distributions on $S$ will correspond via to some «twisted» abelian convolutions on $\mathfrak{s}$ as defined by (3.4) below; with tools from Chapter 4 at hand, (3.4) will be motivated at the beginning of Section 4.3.2.

\footnotetext{
${ }^{1}$ Some authors have named «Rouvière's isomorphism» the map $P \mapsto \beta(j(\partial) P)$. So far so good but it should be noted that it is an isomorphism of algebras in some cases only, e.g. for special symmetric spaces. Generalized Duflo isomorphisms will be discussed in Section 3.3.4
} 
Definition 3.3 Let $e: \Omega \rightarrow \mathbb{R}$ be an analytic function on an open neighborhood $\Omega$ of $(0,0)$ in $\mathfrak{s}^{\prime} \times \mathfrak{s}^{\prime}$, such that:

(i) for all $h \in H$ and $(X, Y) \in \Omega$, one has $(h \cdot X, h \cdot Y) \in \Omega$ and $e(h \cdot X, h \cdot Y)=$ $e(X, Y)$

(ii) for any $H$-invariant distributions $u, v$ and any smooth function $f$ on open neighborhoods of the origin in $\mathfrak{s}$ (with suitable supports), one has

$$
\left\langle\widetilde{u} *_{S} \widetilde{v}, \widetilde{f}\right\rangle=\langle u(X) \otimes v(Y), e(X, Y) f(X+Y)\rangle .
$$

We call such a function an $e$-function of $S$ (associated with the given function $j$ ).

If 1 is an e-function, that is

$$
\widetilde{u} *_{S} \widetilde{v}=\left(u *_{\mathfrak{s}} v\right)^{\sim}
$$

for any $H$-invariant distributions $u, v$ on open neighborhoods of the origin in $\mathfrak{s}$ (with suitable supports), the symmetric space $S$ is said to be special.

No invariance of $f$ is necessary here. A precise assumption about the supports of $u, v, f$ is in order, so that both sides of (3.4) make sense. Let $u, v$ be $H$ invariant distributions on some open subsets of $\mathfrak{s}^{\prime}$ and $f \in C^{\infty}(\mathfrak{s})$. Writing $Z_{0}(X, Y):=X+Y$, we assume that

$$
\left\{\begin{array}{l}
\Omega \cap(\operatorname{supp} u \times \operatorname{supp} v) \cap \operatorname{supp}(f \circ Z) \\
\Omega \cap(\operatorname{supp} u \times \operatorname{supp} v) \cap \operatorname{supp}\left(f \circ Z_{0}\right)
\end{array} \quad\right. \text { are compact. }
$$

Our main applications will be to invariant differential operators: $v$ is then an $H$-invariant distribution supported at the origin of $\mathfrak{s}$ (Example 2 in 3.1.1). Let

$$
\Omega_{1}:=\{X \in \mathfrak{s} \mid(X, 0) \in \Omega\},
$$

an $H$-invariant open subset of $\mathfrak{s}^{\prime}$. Since $Z(X, 0)=Z_{0}(X, 0)=X$ assumption (3.5) becomes: $u$ is an $H$-invariant distribution on an open subset $U$ of $\mathfrak{s}$, $f \in C^{\infty}(\mathfrak{s})$ and $\Omega_{1} \cap \operatorname{supp} u \cap \operatorname{supp} f$ is compact. This support condition is fulfilled, for instance, if $f \in \mathcal{D}\left(\Omega_{1} \cap U\right)$.

Choosing $u=v=\delta_{0}$ in (3.4) shows that $e(0,0)=1$ necessarily.

Example 1. Let $G=S O_{o}(n, 1)$ (the connected component of the identity in $S O(n, 1)), H=S O(n)$ and $j=J^{1 / 2}$. An $e$-function for the real hyperbolic space $H^{n}(\mathbb{R})=G / H$ is

$$
e(X, Y)=A(\|X\|,\|Y\|,\|X+Y\|)^{(n-3) / 2}
$$

where the function $A$ and the norm are defined in Theorem 3.23; in particular $H^{3}(\mathbb{R})$ is special. A hypergeometric factor also appears in $e$ for the other hyperbolic spaces (complex, quaternionian, exceptional).

Example 2. More examples of special spaces are given in Section 3.2. 
We shall prove in Chapter 4 (Theorem 4.12) that any symmetric space admits an $e$-function on some suitably chosen $\Omega$ (Theorem 4.6). But excepting Section 3.7 our purpose in the present chapter is only to develop some consequences of (3.4), taking for granted the existence of an $e$-function of $S$.

Let us modify the left-hand side of (3.4) into a more convenient form. For $X, Y$ in a neighborhood of the origin of $\mathfrak{s}$ let $Z(X, Y) \in \mathfrak{s}^{\prime}$ be defined by

$$
\operatorname{Exp} Z(X, Y)=\exp X \cdot \operatorname{Exp} Y \text {. }
$$

Proposition 3.4 Let $\Omega$ be an open neighborhood of $(0,0)$ in $\mathfrak{s}^{\prime} \times \mathfrak{s}^{\prime}$ on which $e: \Omega \rightarrow \mathbb{R}$ and $Z: \Omega \rightarrow \mathfrak{s}^{\prime}$ are analytic.

(i) Let $u, v, f$ satisfy (3.5). The equality (3.4) in Definition 3.3 is equivalent to the following:

$$
\begin{aligned}
\left\langle u(X) \otimes v(Y), \frac{j(X) j(Y)}{j(Z(X, Y))} f\right. & Z(X, Y))\rangle= \\
& =\langle u(X) \otimes v(Y), e(X, Y) f(X+Y)\rangle .
\end{aligned}
$$

(ii) If $\operatorname{det}_{\mathfrak{s}} \operatorname{Ad} h=1$ for all $h \in H$, an e-function satisfies $e(X, 0)=e(0, Y)=$ 1 for all $X, Y$ such that $(X, 0)$ and $(0, Y)$ belong to $\Omega$.

Remarks. (a) Replacing $u$ by $j^{-1} u, v$ by $j^{-1} v$ and $f$ by $j f$ gives the following variant of (3.6)

$$
\begin{aligned}
& \langle u(X) \otimes v(Y), f(Z(X, Y))\rangle= \\
& \quad=\left\langle u(X) \otimes v(Y), \frac{j(X+Y)}{j(X) j(Y)} e(X, Y) f(X+Y)\right\rangle .
\end{aligned}
$$

Looking at (3.6) or (3.7) we see that, up to the $j$ factors, $e$ plays the same role as the Jacobian of a map transforming $Z(X, Y)$ into $X+Y$; this is the strategy to construct $e$ in Chapter 4.

If $j$ and $\lambda$ are analytic functions on $\mathfrak{s}^{\prime}$, strictly positive and $H$-invariant, normalized by $j(0)=\lambda(0)=1$ and $e(X, Y)$ is an $e$-function associated to $j$, then

$$
\frac{\lambda(X) \lambda(Y)}{\lambda(X+Y)} e(X, Y)
$$

is an $e$-function associated to $\lambda j$. If $e$ itself may be written as $e(X, Y)=$ $\lambda(X+Y) / \lambda(X) \lambda(Y)$ for some $\lambda$, replacing $j$ by $\lambda j$ will turn $S$ into a special space.

(b) The assumption of (ii) holds true if $G / H$ has a $G$-invariant measure and $H$ is connected, in particular if $H$ is compact connected.

Proof. (i) The functions $\frac{j(X) j(Y)}{j(Z(X, Y))} f(Z(X, Y))$ and $e(X, Y) f(X+Y)$ are smooth on $(X, Y) \in \Omega$ and, assuming (3.5), both sides of (3.6) are welldefined. The left-hand side is $\langle u(X), \varphi(X)\rangle$ with

$$
\varphi(X):=j(X)\left\langle v(Y), \frac{j(Y)}{j(Z(X, Y))} f(Z(X, Y))\right\rangle .
$$


The variables $X, Y$ remain in $\mathfrak{s}^{\prime}$ and repeated use of the definition of $\sim$ gives $\langle u, \varphi\rangle=\langle\widetilde{u}, \widetilde{\varphi}\rangle$ with

$$
\begin{aligned}
\widetilde{\varphi}(\operatorname{Exp} X) & =j(X)^{-1} \varphi(X)=\langle v(Y), j(Y) \widetilde{f}(\operatorname{Exp} Z(X, Y))\rangle \\
& =\left\langle v(Y), j(Y)\left(\tilde{f} \circ \tau\left(e^{X}\right)\right)(\operatorname{Exp} Y)\right\rangle=\left\langle\widetilde{v}, \tilde{f} \circ \tau\left(e^{X}\right)\right\rangle .
\end{aligned}
$$

The left-hand side of (3.6) is finally $\langle\widetilde{u}(g H),\langle\widetilde{v}, \widetilde{f} \circ \tau(g)\rangle\rangle$, that is

$$
\left\langle\widetilde{u} *_{S} \widetilde{v}, \widetilde{f}\right\rangle=\left\langle u(X) \otimes v(Y), \frac{j(X) j(Y)}{j(Z(X, Y))} f(Z(X, Y))\right\rangle
$$

as claimed.

(ii) Here the Lebesgue measure $d X$ of $\mathfrak{s}$ defines an $H$-invariant distribution on $\mathfrak{s}$. Applying (3.6) to $u=d X$ and $v=\delta_{0}$ (the Dirac measure of $\mathfrak{s}$ ) we obtain, in view of $Z(X, 0)=X$ and $j(0)=1$,

$$
\int_{\mathfrak{s}} f(X)(1-e(X, 0)) d X=0
$$

for any $f \in \mathcal{D}\left(\Omega_{1}\right)$, hence $e(X, 0)=1$ if $(X, 0) \in \Omega$. Similarly $u=\delta_{0}$ and $v=d Y$ give $e(0, Y)=1$.

\subsection{Special Symmetric Spaces}

Let us study first the nice case when convolutions of $H$-invariant distributions on $S$ correspond exactly, by the transfer map , to classical abelian convolutions on its tangent space $\mathfrak{s}$, as was the case for Lie groups in Chapter 1 . We call special such symmetric spaces (Definition 3.3). This property essentially implies $j=J^{1 / 2}$; see Propositions 3.6 and 4.23 for precise statements.

As shown by Propositions 3.5 and 3.6, $H$-invariant analysis on special symmetric spaces boils down to classical abelian analysis on the tangent space. The following spaces are special (with $j=J^{1 / 2}$ ):

- all symmetric spaces $G / H$ with $G$ solvable (Theorem 4.22 )

- all strongly symmetric spaces (Definition 4.21 and Theorem 4.22), e.g. spaces of the form $G_{\mathbb{C}} / G_{\mathbb{R}}$ or Lie groups viewed as symmetric spaces

- a Riemannian symmetric space of the noncompact type $G / K$ is special if and only if $G$ admits a complex structure (Proposition 3.7).

As in Section 3.1.2, $\Omega$ denotes an open neighborhood of $(0,0)$ in $\mathfrak{s}^{\prime} \times \mathfrak{s}^{\prime}$, invariant under $(X, Y) \mapsto(h \cdot X, h \cdot Y)$ for $h \in H$, on which $Z$ is analytic. Let $\Omega_{1}:=\{X \in \mathfrak{s} \mid(X, 0) \in \Omega\} \subset \mathfrak{s}^{\prime}$ and $Z_{0}(X, Y):=X+Y$. The map $P \mapsto \widetilde{P}$ is defined in 3.1.1. 
Proposition 3.5 Assume $S$ is a special symmetric space. Then

(i) Let $u, v$ be $H$-invariant distributions on open subsets of $\mathfrak{s}$ and $f \in C^{\infty}(\mathfrak{s})$ such that (3.5) holds. Then

$$
\left\langle\widetilde{u} *_{S} \widetilde{v}, \widetilde{f}\right\rangle=\left\langle\widetilde{u *_{\mathfrak{s}} v}, \widetilde{f}\right\rangle=\left\langle u *_{\mathfrak{s}} v, f\right\rangle .
$$

If $P \in \mathbb{D}(\mathfrak{s})^{H}$ and $u$ is an $H$-invariant distribution on an open subset $U$ of $\mathfrak{s}$,

$$
\widetilde{{ }^{t} P u}={ }^{t} \widetilde{P} \widetilde{u}
$$

an equality of distributions on $\operatorname{Exp}\left(\Omega_{1} \cap U\right)$.

(ii) The map $P \mapsto \widetilde{P}$ is an algebra isomorphism of $\mathbb{D}(\mathfrak{s})^{H}$ onto $\mathbb{D}(S)$.

(iii) Any non-zero differential operator in ${ }^{t} \mathbb{D}(S)$ has an $H$-invariant fundamental solution on $\Omega_{1}$ and is locally solvable.

The transfer map reduces invariant differential equations on a special space to equations with constant coefficients on its tangent space.

As noted at the end of $2.1,{ }^{t} \mathbb{D}(S)=\mathbb{D}(S)$ if $S$ has a $G$-invariant mesaure.

Proof. (i) follows from Definition 3.3 with $e=1$ on $\Omega$ and the support condition (3.5). The second equality is obtained by taking $v={ }^{t} P \delta_{0}$, in view of $(3.2)$.

(ii) Taking now $u={ }^{t} Q \delta_{0}$ with $Q \in \mathbb{D}(\mathfrak{s})^{H}$ we infer that ${ }^{t} \widetilde{P^{t} Q \delta_{0}}={ }^{t} \widetilde{P}{ }^{t} \widetilde{Q} \delta$, hence ${ }^{t}(\widetilde{Q P})={ }^{t}(\widetilde{Q} \widetilde{P})$ by $(3.1)$ and the conclusion.

(iii) By (ii) any operator in ${ }^{t} \mathbb{D}(S)$ is ${ }^{t} \widetilde{P}$ for some $P \in \mathbb{D}(\mathfrak{s})^{H}$. The differential operator ${ }^{t} P$ has constant coefficients and is $H$-invariant on $\mathfrak{s}$; by a result due to Raïs [40] it admits an $H$-invariant (and tempered) fundamental solution $E \in \mathcal{D}^{\prime}(\mathfrak{s})^{H}$. Restricting it to $\Omega_{1}$ we obtain $E \in \mathcal{D}^{\prime}\left(\Omega_{1}\right)^{H}$ such that ${ }^{t} \widetilde{P} \widetilde{E}=$ $\widetilde{\delta_{0}}=\delta$ on $\operatorname{Exp} \Omega_{1}$ by (ii). The local solvability follows in view of (2.2).

We now discuss, under some stronger assumptions, the meaning of the convolution equality for special symmetric spaces. In order to avoid repetitions we shall use results proved for general symmetric spaces in Section 3.3, specialized here to the case $e=1$. Let $\mathcal{E}^{\prime}(\mathfrak{s})^{H}$ denote the space of $H$-invariant distributions on $\mathfrak{s}$ with compact support and, for $\xi \in \mathfrak{s}^{*}$ (the dual of $\mathfrak{s}$ ),

$$
\widehat{u}(\xi):=\left\langle u(X), e^{-i\langle\xi, X\rangle}\right\rangle
$$

the classical Fourier transform of $u \in \mathcal{E}^{\prime}(\mathfrak{s})^{H}$. Recall that an $H$-invariant smooth function $\varphi$ on a symmetric space $S=G / H, H$ compact, is called a spherical function if $\varphi(o)=1$ and $\varphi$ is an eigenfunction of all differential operators in $\mathbb{D}(S)$. Spherical functions are characterized by the functional equation ([28] p. 400): for all $g, g^{\prime} \in G$

$$
\varphi(g H) \varphi\left(g^{\prime} H\right)=\int_{H} \varphi\left(g h g^{\prime} H\right) d h .
$$


Proposition 3.6 Assume $H$ is compact, Exp $: \mathfrak{s} \rightarrow S$ is a global diffeomorphism onto and $j$ is analytic and strictly positive on $\mathfrak{s}$. The following properties of the Riemannian symmetric space $S=G / H$ are equivalent:

(i) $\widetilde{u} *_{S} \widetilde{v}=\widetilde{u *_{\mathfrak{s}} v}$ on $S$ for all $u, v \in \mathcal{E}^{\prime}(\mathfrak{s})^{H}$

(ii) for every $\xi \in \mathfrak{s}^{*}$ there exists a spherical function $\varphi_{\xi}$ of $S$ such that $\left\langle\widetilde{u}, \varphi_{\xi}\right\rangle=\widehat{u}(\xi)$ for all $u \in \mathcal{E}^{\prime}(\mathfrak{s})^{H}$.

These properties imply $j(X) j(-X)=J(X)$ for $X \in \mathfrak{s}$, and $j=J^{1 / 2}$ if $j$ is even.

Here the transfer map reduces $H$-invariant harmonic analysis on $S$ to classical Fourier analysis on the tangent space.

Proof. (i) $\Rightarrow$ (ii). Assuming (i) we have $\widetilde{{ }^{t} P u}={ }^{t} \widetilde{P} \widetilde{u}$ for all $u \in \mathcal{E}^{\prime}(\mathfrak{s})^{H}$ and, by the proof of Theorem $3.8(i i), \widetilde{P} \widetilde{f}=\widetilde{P f}$ on $S$ for all $P \in \mathbb{D}(\mathfrak{s})^{H}$ and all $H$-invariant smooth functions $f$ on $\mathfrak{s}$. We apply this to the integral $f_{\xi}(X):=$ $\int_{H} e^{-i\langle\xi, h \cdot X\rangle} d h$ (generalized Bessel function), which is an eigenfunction of all operators $P \in \mathbb{D}(\mathfrak{s})^{H}$ namely $P\left(\partial_{X}\right) f_{\xi}=P(-i \xi) f_{\xi}$. Thus $\varphi_{\xi}:=\widetilde{f}_{\xi}$ is a spherical function on $S$ and, for $u \in \mathcal{E}^{\prime}(\mathfrak{s})^{H},\left\langle\widetilde{u}, \varphi_{\xi}\right\rangle=\left\langle u, f_{\xi}\right\rangle=\widehat{u}(\xi)$.

(ii) $\Rightarrow($ i). Assuming (ii) we have

$$
\begin{aligned}
\left\langle\widetilde{u *_{\mathfrak{s}} v}, \varphi_{\xi}\right\rangle & =\widetilde{u *_{\mathfrak{s}} v}(\xi)=\widehat{u}(\xi) \widehat{v}(\xi) \\
& =\left\langle\widetilde{u}, \varphi_{\xi}\right\rangle\left\langle\widetilde{v}, \varphi_{\xi}\right\rangle=\left\langle\widetilde{u} *_{S} \widetilde{v}, \varphi_{\xi}\right\rangle,
\end{aligned}
$$

as follows from the functional equation of spherical functions and the definition of $*_{S}$. Let $w \in \mathcal{E}^{\prime}(\mathfrak{s})^{H}$ be defined by $\widetilde{w}=\widetilde{u *_{\mathfrak{s}} v}-\widetilde{u} *_{S} \widetilde{v}$. Then $\left\langle\widetilde{w}, \varphi_{\xi}\right\rangle=\widehat{w}(\xi)=0$ for all $\xi \in \mathfrak{s}^{*}$, whence $w=0$ and our claim.

Finally let us write $(i)$ at the origin for distributions $u(X) d X, v(X) d X$, with $u, v \in \mathcal{D}(\mathfrak{s})^{H}$. By Example 1 in 3.1.1 we have

$$
\widetilde{u d X}=U d x \text { with } U(\operatorname{Exp} X)=\left(j J^{-1}\right)(X) u(X)
$$

and (see 2.1)

$$
\begin{aligned}
\widetilde{u d X} *_{S} \widetilde{v d X} & =U d x *_{S} V d x=W_{1} d x \\
\text { with } W_{1}(x) & =\int_{S} U(g H) V\left(g^{-1} \cdot x\right) d(g H) .
\end{aligned}
$$

On the other hand, by (i),

$$
\begin{aligned}
\widetilde{u d X} *_{S} \widetilde{v d X} & =\left(u d X *_{\mathfrak{s}} v d X\right)^{\sim}=\widetilde{w d X}=W_{2} d x \\
\text { with } W_{2}(\operatorname{Exp} X) & =\left(j J^{-1}\right)(X) \int_{\mathfrak{s}} u(Y) v(X-Y) d Y .
\end{aligned}
$$

Writing $W_{1}(o)=W_{2}(o)$ and remembering $j(0)=J(0)=1, J(-X)=J(X)$, we obtain

$$
\int_{\mathfrak{s}} U(\operatorname{Exp} X) V(\operatorname{Exp}(-X)) J(X) d X=\int_{\mathfrak{s}} u(X) v(-X) d X,
$$


that is

$$
\int_{\mathfrak{s}} u(X) v(-X)\left(j(X) j(-X) J(X)^{-1}-1\right) d X=0
$$

for all $u, v \in \mathcal{D}(\mathfrak{s})^{H}$. Taking $v(-X)=1$ on $\operatorname{supp} u$, the conclusion easily follows since $j(X) j(-X) J(X)^{-1}$ is $H$-invariant (see Lemma 3.15).

The next proposition considers the case of a Riemannian symmetric space of the noncompact type. We use here the semisimple notation explained in Section 0.5.

Proposition 3.7 Let $G$ be a noncompact connected simple Lie group with finite center and $K$ a maximal compact subgroup. With transfer $\sim$ defined by $j=J^{1 / 2}$, the symmetric space $S=G / K$ is special if and only if $G$ admits a complex structure.

Proof. (i) Assume $S$ is special and let $\Delta$, resp. $L$, denote the Laplacian on $\mathfrak{p}$, resp. $S$. By Theorem $3.8(i i)$ below we have $\widetilde{\Delta f}=\widetilde{\Delta f}$ (in a neighborhood of the origin) for any $K$-invariant function $f$, and Proposition 3.11 ( $i i$ ) gives $\widetilde{\Delta}=L+c$ with $c=\Delta j(0)$. Applying this to $f=j$ we obtain $\widetilde{j}=1$, $\widetilde{\Delta j}=L 1+c=c \widetilde{j}$, hence $\Delta j=c j$ on $\mathfrak{p}$ by analytic continuation. Here

$$
j(H)=J(H)^{1 / 2}=\prod_{\alpha}\left(\frac{\operatorname{sh} \alpha(H)}{\alpha(H)}\right)^{m_{\alpha} / 4}
$$

for $H \in \mathfrak{a}$ (product over all positive and negative roots $\alpha$ ). When restricted to the open set $\mathfrak{a}^{\prime}$ of regular elements in $\mathfrak{a}$ the $K$-invariant equality $\Delta j=c j$ implies, by means of the radial part of $\Delta$ for the adjoint action of $K$ on $\mathfrak{p}$ ([28] p. 270):

$$
\left(\Delta_{\mathfrak{a}}+\frac{1}{2} \sum_{\alpha} m_{\alpha} \alpha(H)^{-1} H_{\alpha}\right) j(H)=c j(H), H \in \mathfrak{a}^{\prime},
$$

with a sum over all roots; $\Delta_{\mathfrak{a}}$ is the Laplacian on $\mathfrak{a}$ and $H_{\alpha} \in \mathfrak{a}$, defined by $\left\langle H_{\alpha}, H\right\rangle=\alpha(H)$ for all $H \in \mathfrak{a}$, is viewed here as a constant coefficients differential operator on $\mathfrak{a}$.

Let $\left(H_{i}\right)$ be an orthonormal basis of $\mathfrak{a}$ and $\partial_{i}$ the partial derivative with respect to the $i$-th coordinate; thus $H_{\alpha}=\sum_{i} \alpha_{i} \partial_{i}$ with $\alpha_{i}=\alpha\left(H_{i}\right)$ and $\Delta_{\mathfrak{a}}=\sum_{i} \partial_{i}^{2}$. Then $j^{-1} \Delta j=c$ restricted to $\mathfrak{a}^{\prime}$ becomes

$$
\sum_{i} \partial_{i}\left(j^{-1} \partial_{i} j\right)+\sum_{i}\left(j^{-1} \partial_{i} j\right)^{2}+\frac{1}{2} \sum_{i, \alpha} m_{\alpha} \alpha(H)^{-1} \alpha_{i}\left(j^{-1} \partial_{i} j\right)=c .
$$

Since $j^{-1} \partial_{i} j=\frac{1}{4} \sum_{\alpha} m_{\alpha}\left(\operatorname{coth} \alpha(H)-\alpha(H)^{-1}\right) \alpha_{i}$, elementary calculations 
lead to

$$
\begin{aligned}
4 \sum_{\alpha} m_{\alpha}\left(\alpha(H)^{-2}\right. & \left.-(\operatorname{sh} \alpha(H))^{-2}\right)|\alpha|^{2}+ \\
& +\left|\sum_{\alpha} m_{\alpha}(\operatorname{coth} \alpha(H)) \alpha\right|^{2}-\left|\sum_{\alpha} m_{\alpha} \alpha(H)^{-1} \alpha\right|^{2}=16 c
\end{aligned}
$$

for all $H \in \mathfrak{a}^{\prime}$, with $|\lambda|^{2}:=\left\langle H_{\lambda}, H_{\lambda}\right\rangle=\sum_{i} \lambda_{i}^{2}$ for $\lambda \in \mathfrak{a}^{*}$. Replacing $H$ by $t H$, the asymptotic expansion as $t \rightarrow \infty$ implies $c=|\rho|^{2}$ with $\rho=\frac{1}{2} \sum_{\alpha>0} m_{\alpha} \alpha$ and, for the $t^{-2}$ terms,

$$
4 \sum_{\alpha} m_{\alpha} \alpha(H)^{-2}|\alpha|^{2}=\left|\sum_{\alpha} m_{\alpha} \alpha(H)^{-1} \alpha\right|^{2} .
$$

Fix an indivisible root $\alpha$ and let $\beta$ denote any root non-proportional to $\alpha$ (assuming such $\beta$ exist). There exists $H_{0} \in \mathfrak{a}$ such that $\alpha\left(H_{0}\right)=0$ and $\left|\beta\left(H_{0}\right)\right|>1$ for all $\beta$ 's: one may take $H_{1} \in \operatorname{ker} \alpha$ outside the finite number of $(d-2)$-planes $\operatorname{ker} \alpha \cap \operatorname{ker} \beta$ (with $d=\operatorname{dim} \mathfrak{a}$ ), and a suitable multiple of $H_{1}$ will do. Replacing $H_{0}$ by $H:=H_{0}+s|\alpha|^{-2} H_{\alpha}$ with $s>0$ small enough, we obtain $\alpha(H)=s$ and $|\beta(H)|>1$ for all $\beta$ 's. In the above equality, written at this point $H$, we separate $\pm \alpha$ and $\pm 2 \alpha$ from the other roots $\beta$ :

$$
\begin{aligned}
8\left(m_{\alpha}+\right. & \left.m_{2 \alpha}\right)|\alpha|^{2} s^{-2}+4 \sum_{\beta} m_{\beta} \beta(H)^{-2}|\beta|^{2}=4\left(m_{\alpha}+m_{2 \alpha}\right)^{2}|\alpha|^{2} s^{-2}+ \\
& +4\left(m_{\alpha}+m_{2 \alpha}\right) \sum_{\beta} m_{\beta} \beta(H)^{-1}\langle\alpha, \beta\rangle s^{-1}+\left|\sum_{\beta} m_{\beta} \beta(H)^{-1} \beta\right|^{2} .
\end{aligned}
$$

The coefficients including $\beta(H)^{-1}$ remain bounded as $s \rightarrow 0$. The $s^{-2}$ terms show that $m_{\alpha}+m_{2 \alpha}=2$, therefore $m_{\alpha}=m_{2 \alpha}=1$ or else $m_{\alpha}=2$ and $m_{2 \alpha}=0$. The former case is impossible because $\left[\mathfrak{g}_{\alpha}, \mathfrak{g}_{\alpha}\right]=\mathfrak{g}_{2 \alpha}([27]$ p. 408). Thus $m_{\alpha}=2$ and $m_{2 \alpha}=0$ for all indivisible roots $\alpha$. By Araki's results for simple Lie algebras this implies that $\mathfrak{g}$ admits a complex structure ([27] p. $531)$.

If all roots are proportional to $\alpha$, there are no $\sum_{\beta}$ 's in the above equation whence $m_{\alpha}+m_{2 \alpha}=2$ and the same conclusion.

(ii) The converse follows from Theorem 4.22 (ii) or from Proposition 3.6 (ii) and the expression of spherical functions of $G / K$ for $G$ complex ([28] p. 425).

\section{3 $e$-Functions and Invariant Differential Op- erators}

Specializing Definition 3.3 of $e$-functions to distributions supported at the origin (Example 2 in 3.1.1), we shall now derive some interesting relations 
between $e$ and the algebra of $G$-invariant differential operators on a general symmetric space $S=G / H$. The philosophy of this section and the next one is that several important objects in $H$-invariant analysis on this space are determined by the algebraic structure of $S(\mathfrak{s})^{H}$ (the commutative algebra of $H$-invariant elements in the symmetric algebra of $\mathfrak{s})$ together with an $e$ function. We first recall a few elementary facts on symmetric algebras.

\subsubsection{The Fischer Product}

Let $V$ denote a finite dimensional vector space over $\mathbb{R}$ and $V^{*}$ its dual space. The (complexified) symmetric algebra $S(V)$ is canonically isomorphic to the algebra of (complex valued) polynomial functions on $V^{*}$ and to the algebra $\mathbb{D}(V)$ of linear differential operators with (complex) constant coefficients on $V$. Writing $P$ or $P(\xi)$ or $P\left(\partial_{X}\right)$ accordingly an element of this algebra (with $\left.\xi \in V^{*}, X \in V\right)$ we have

$$
P\left(\partial_{X}\right)\left(e^{<\xi, X>}\right)=P(\xi) e^{<\xi, X>} .
$$

Similarly an element $Q$ of $S\left(V^{*}\right)$ is a polynomial function on $V$ or a differential operator on $V^{*}$. The duality between $V$ and $V^{*}$ extends to a duality between $S(V)$ and $S\left(V^{*}\right)$ by means of the nondegenerate bilinear form (the Fischer product)

$$
\langle P \mid Q\rangle=\langle P(\xi) \mid Q(X)\rangle:=\left.P\left(\partial_{X}\right) Q(X)\right|_{X=0} .
$$

Let $\left(e_{1}, \ldots, e_{n}\right)$, resp. $\left(e_{1}^{*}, \ldots, e_{n}^{*}\right)$, denote dual bases of $V$, resp. $V^{*}$, and $X=\sum X_{i} e_{i}, \xi=\sum \xi_{j} e_{j}^{*}$ the corresponding decompositions. Then, writing $P(\xi)=\sum a_{\alpha} \xi^{\alpha}, Q(X)=\sum b_{\beta} X^{\beta}$ in multi-index notation $\alpha=\left(\alpha_{1}, \ldots, \alpha_{n}\right) \in$ $\mathbb{N}^{n}$, we have

$$
\left.P\left(\partial_{X}\right) Q(X)\right|_{X=0}=\left.Q\left(\partial_{\xi}\right) P(\xi)\right|_{\xi=0} .
$$

since both sides equal $\sum_{\alpha} \alpha ! a_{\alpha} b_{\alpha}$. Let us call «finite» a linear form on $S(V)$ which vanishes on all homogeneous elements of $S(V)$ of degree greater than some value; the map $Q \mapsto\langle. \mid Q\rangle$ allows to identify $S\left(V^{*}\right)$ with the space of finite linear forms on $S(V)$.

Replacing $Q$ by the product of two elements $Q, R \in S\left(V^{*}\right)$ in (3.10) we obtain

$$
\langle P \mid Q R\rangle=\left.R\left(\partial_{\xi}\right)\left(Q\left(\partial_{\xi}\right) P(\xi)\right)\right|_{\xi=0}=\left\langle Q\left(\partial_{\xi}\right) P(\xi) \mid R(X)\right\rangle
$$

by (3.10) again, that is

$$
\langle P(\xi) \mid Q(X) R(X)\rangle=\left\langle Q\left(\partial_{\xi}\right) P(\xi) \mid R(X)\right\rangle .
$$

Thus $Q\left(\partial_{\xi}\right)$ is the transpose of the multiplication operator by $Q(X)$ with respect to the Fischer product.

More generally (3.11) still holds if $Q$ is a formal series and $R$ a smooth function near the origin of $V$, since the formula only involves a finite number of derivatives of $R$ at the origin. 


\subsubsection{Explicit Transfer of Invariant Differential Opera- tors}

We retain the notation of 3.1. Our next theorem gives an explicit relation, by means of $e$, between an $H$-invariant constant coefficients differential operator $P \in \mathbb{D}(\mathfrak{s})^{H}$ on the tangent space $\mathfrak{s}$ and the corresponding $G$-invariant differential operator $\widetilde{P} \in \mathbb{D}(S)$ on the symmetric space. As usual ${ }^{t}$ denotes the transposition of differential operators.

Theorem 3.8 Let $e: \Omega \rightarrow \mathbb{R}$ be an e-function of a symmetric space $S=$ $G / H$ and $\Omega_{1}=\{X \in \mathfrak{s} \mid(X, 0) \in \Omega\}$.

(i) For $P \in \mathbb{D}(\mathfrak{s})^{H}$ the equation

$$
P_{e}\left(X, \partial_{X}\right) f(X):=\left.P\left(\partial_{Y}\right)(e(X, Y) f(X+Y))\right|_{Y=0},
$$

$f \in C^{\infty}\left(\Omega_{1}\right)$, defines an $H$-invariant differential operator $P_{e}$ with analytic coefficients on $\Omega_{1}$ and symbol $P_{e}(X, \xi)=e\left(X, \partial_{\xi}\right) P(\xi)$. For any $H$-invariant distribution $u$ on $\Omega_{1}$

$$
{ }^{t} \widetilde{P} \widetilde{u}=\left({ }^{t} P_{e}\left(X, \partial_{X}\right) u\right)^{\sim} \text { on } \operatorname{Exp} \Omega_{1} .
$$

(ii) If $H$ is compact and $f$ is any $H$-invariant smooth function on $\Omega_{1}$

$$
\widetilde{P} \tilde{f}=\left(P_{e}\left(X, \partial_{X}\right) f\right)^{\sim} \text { on } \operatorname{Exp} \Omega_{1} \text {. }
$$

Remarks. (a) The assumptions on $\Omega$ (Definition 3.3) imply that $\Omega_{1}$ is an $H$-invariant open subset of $\mathfrak{s}^{\prime}$.

(b) If $\left(X_{1}, \ldots, X_{n}\right)$ are coordinates with respect to a basis of $\mathfrak{s}$ and $\left(\xi_{1}, \ldots, \xi_{n}\right)$ the dual coordinates on $\mathfrak{s}^{*}$ we have, in multi-index notation,

$$
\begin{aligned}
& e(X, Y)=\sum_{\alpha, \beta \in \mathbb{N}^{n}} \frac{1}{\alpha ! \beta !} \partial_{X}^{\alpha} \partial_{Y}^{\beta} e(0,0) X^{\alpha} Y^{\beta} \\
& P_{e}(X, \xi)=\sum_{\beta} \frac{1}{\beta !} \partial_{Y}^{\beta} e(X, 0) \partial_{\xi}^{\beta} P(\xi)=\sum_{\alpha, \beta} \frac{1}{\alpha ! \beta !} \partial_{X}^{\alpha} \partial_{Y}^{\beta} e(0,0) X^{\alpha} \partial_{\xi}^{\beta} P(\xi),
\end{aligned}
$$

with finite summation over $\beta$ in the latter equations.

(c) Theorem 4.16 below extends the result of (ii) to arbitrary symmetric spaces, for a specific choice of $e$.

(d) In view of (ii) finding the radial part of $\widetilde{P}$ for the action of $H$ on $S$ is equivalent to the same problem for $P_{e}$ on $\mathfrak{s}$.

Proof. (i) In view of (3.1) (3.2) Definition 3.3 implies, with $v={ }^{t} P \delta_{0}$,

$$
\left\langle\widetilde{u} *_{S}{ }^{t} \widetilde{P} \delta, \widetilde{f}\right\rangle=\left\langle u(X),\left\langle{ }^{t} P \delta_{0}(Y), e(X, Y) f(X+Y)\right\rangle\right\rangle
$$


for any test function $f$ on $\Omega_{1}$, that is

$$
\begin{aligned}
\left\langle{ }^{t} \widetilde{P} \widetilde{u}, \tilde{f}\right\rangle & =\left\langle u(X),\left.P\left(\partial_{Y}\right)(e(X, Y) f(X+Y))\right|_{Y=0}\right\rangle \\
& =\left\langle{ }^{t} P_{e}\left(X, \partial_{X}\right) u(X), f(X)\right\rangle=\left\langle\left({ }^{t} P_{e}\left(X, \partial_{X}\right) u\right) \widetilde{f}\right\rangle .
\end{aligned}
$$

Besides, by (3.11) with $V=\mathfrak{s}, X$ fixed and $Y$ as the variable,

$$
\begin{aligned}
P_{e}\left(X, \partial_{X}\right) f(X) & =\left.P\left(\partial_{Y}\right)(e(X, Y) f(X+Y))\right|_{Y=0} \\
& =\langle P \mid e(X, .) f(X+.)\rangle=\langle e(X, \partial) P \mid f(X+.)\rangle,
\end{aligned}
$$

thus $P_{e}\left(X, \partial_{X}\right)$ is obtained by replacing $\xi$ by $\partial_{X}$ in $P_{e}(X, \xi)=e\left(X, \partial_{\xi}\right) P(\xi)$, hence (3.12). The $H$-invariance of $P_{e}$ is an immediate consequence of the invariance of $P$ and $e$.

(ii) By (i) we have $\langle\widetilde{u}, \widetilde{P} \widetilde{f}\rangle=\left\langle\widetilde{u}, \widetilde{P_{e} f}\right\rangle$ for all $f \in \mathcal{D}\left(\Omega_{1}\right)$. Assuming $H$ is compact and $f$ is $H$-invariant, $P_{e} f$ is $H$-invariant too and the $H$-invariant function $\widetilde{P} \widetilde{f}-\widetilde{P_{e} f}$ is annihilated by any $H$-invariant distribution on $\operatorname{Exp} \Omega_{1}$. This function must therefore vanish identically, as follows from the existence of $H$-invariant means (Lemma 3.15 below). Thus $\widetilde{P} \widetilde{f}=\widetilde{P_{e} f}$ if $\operatorname{supp} f$ is compact, and the result extends to any $H$-invariant $f$ on $\Omega_{1}$ by invariant truncation.

The next result shows how $e$ and the map $\sim$ defined by (3.3) relate the structures of the algebras $\mathbb{D}(\mathfrak{s})^{H}=S(\mathfrak{s})^{H}$ (the $H$-invariant elements in the symmetric algebra of the tangent space) and $\mathbb{D}(S)$ (the $G$-invariant differential operators on the symmetric space).

Theorem 3.9 Let $e(X, Y)$ be an e-function of a symmetric space $S=G / H$. Then, for any $P, Q \in \mathbb{D}(\mathfrak{s})^{H}$,

$$
\widetilde{P} \circ \widetilde{Q}=\widehat{P \times Q}
$$

where the product $\times$ on $\mathbb{D}(\mathfrak{s})^{H}$ is defined by

$$
(P \times Q)(\xi):=\left.e\left(\partial_{\xi}, \partial_{\eta}\right)(P(\xi) Q(\eta))\right|_{\xi=\eta}=\left.\left(Q_{e}\left(\partial_{\xi}, \eta\right) P(\xi)\right)\right|_{\xi=\eta},
$$

with $\xi, \eta \in \mathfrak{s}^{*}$. Thus the map $P \mapsto \widetilde{P}$ is an order preserving isomorphism of algebras of $\left(\mathbb{D}(\mathfrak{s})^{H}, \times\right)$ onto $(\mathbb{D}(S), \circ)$.

The differential operator $Q_{e}\left(\partial_{\xi}, \eta\right)$ is obtained from $Q_{e}(X, \eta)$ in the previous theorem by substituting $\partial_{\xi}$ for $X$, so that

$$
Q_{e}\left(X, \partial_{X}\right) e^{<\xi, X>}=Q_{e}(X, \xi) e^{<\xi, X>}=\left.Q_{e}\left(\partial_{\xi}, \eta\right) e^{<\xi, X>}\right|_{\eta=\xi} .
$$

In dual coordinates $\left(X_{1}, \ldots, X_{n}\right),\left(\xi_{1}, \ldots, \xi_{n}\right)$ on $\mathfrak{s}$ and $\mathfrak{s}^{*}$ we have the expansion (finite sum)

$$
\begin{aligned}
(P \times Q)(\xi) & =\sum_{\alpha, \beta \in \mathbb{N}^{n}} \frac{1}{\alpha ! \beta !} \partial_{X}^{\alpha} \partial_{Y}^{\beta} e(0,0) \partial_{\xi}^{\alpha} P(\xi) \partial_{\xi}^{\beta} Q(\xi) \\
& =P(\xi) Q(\xi)+\text { lower order terms }
\end{aligned}
$$


since $e(0,0)=1$. The product $\times$ is thus a deformation of the classical product in the symmetric algebra; of course $P \times Q=P Q$ if the symmetric space is special.

Proof. We already know from 3.1.1 that $\sim$ is an order preserving isomorphism of vector spaces, whence the existence of a unique $R \in \mathbb{D}(\mathfrak{s})^{H}$ such that $\widetilde{P} \circ \widetilde{Q}=\widetilde{R}$. To make $R$ explicit we may apply Theorem 3.8 with $u$ supported at the origin, or compute directly as follows.

Let $f$ be a smooth function on $\mathfrak{s}$ with support in a neighborhood of the origin. Then

$$
\begin{aligned}
R(\partial) f(0) & =\left\langle{ }^{t} R \delta_{0}, f\right\rangle=\left\langle\widetilde{{ }^{t} R \delta_{0}}, \widetilde{f}\right\rangle=\left\langle{ }^{t} \widetilde{R} \delta, \widetilde{f}\right\rangle \\
& =\left\langle{ }^{t} \widetilde{Q}\left({ }^{t} \widetilde{P} \delta\right), \widetilde{f}\right\rangle=\left\langle{ }^{t} \widetilde{P} \delta *{ }^{t} \widetilde{Q} \delta, \widetilde{f}\right\rangle \\
& =\left\langle{ }^{t} P \delta_{0}(X) \otimes{ }^{t} Q \delta_{0}(Y), e(X, Y) f(X+Y)\right\rangle \\
& =\left.P\left(\partial_{X}\right) Q\left(\partial_{Y}\right)(e(X, Y) f(X+Y))\right|_{X=Y=0}
\end{aligned}
$$

by the definition of $e$. The latter expression is a Fischer product on the space $V=\mathfrak{s} \times \mathfrak{s}$, namely

$$
\begin{aligned}
R(\partial) f(0) & =\langle P(\xi) Q(\eta) \mid e(X, Y) f(X+Y)\rangle \\
& =\left\langle e\left(\partial_{\xi}, \partial_{\eta}\right) P(\xi) Q(\eta) \mid f(X+Y)\right\rangle
\end{aligned}
$$

in view of (3.11). The derivatives $\partial_{X}$ and $\partial_{Y}$ have the same effect on $f(X+Y)$ and, going back to Fischer products on $\mathfrak{s}$, we have proved

$$
\langle R(\xi) \mid f(X)\rangle=\left\langle\left. e\left(\partial_{\xi}, \partial_{\eta}\right) P(\xi) Q(\eta)\right|_{\xi=\eta} \mid f(X)\right\rangle,
$$

which gives the first expression of $R=P \times Q$. The second follows easily since $Q_{e}(X, \eta)=e\left(X, \partial_{\eta}\right) Q(\eta)$ (Theorem 3.8), whence $Q_{e}\left(\partial_{\xi}, \eta\right)=e\left(\partial_{\xi}, \partial_{\eta}\right) Q(\eta)$ and

$$
e\left(\partial_{\xi}, \partial_{\eta}\right) P(\xi) Q(\eta)=Q_{e}\left(\partial_{\xi}, \eta\right) P(\xi) .
$$

Remarks. (a) The composition $\circ$ of differential operators is associative and Theorem 3.9 implies that $\times$ is an associative law on $\mathbb{D}(\mathfrak{s})^{H}$. The derivatives of $e$ at the origin must therefore satisfy some (non-trivial) identities.

Going the other way around, $x$ is clearly commutative if $e$ is a symmetric function $(e(X, Y)=e(Y, X))$; the algebra $\mathbb{D}(S)$ is then commutative. This holds true for a large class of symmetric spaces, as will be shown in Theorem $4.24(i i)$.

(b) Let $R=P \times Q$. Then ${ }^{t} R_{e}\left(X, \partial_{X}\right) u={ }^{t} Q_{e}\left(X, \partial_{X}\right) \circ{ }^{t} P_{e}\left(X, \partial_{X}\right) u$ for all $H$-invariant distributions $u$ on $\Omega_{1}$ by combination of both theorems and, if $H$ is compact, $R_{e}\left(X, \partial_{X}\right) f=P_{e}\left(X, \partial_{X}\right) \circ Q_{e}\left(X, \partial_{X}\right) f$ for all $H$-invariant functions $f$ on $\Omega_{1}$.

Corollary 3.10 Assume the algebra $\mathbb{D}(\mathfrak{s})^{H}$ (with its usual product) has a finite system of generators $P_{1}, \ldots, P_{l}$. Then $\widetilde{P_{1}}, \ldots, \widetilde{P}_{l}$ generate the algebra $(\mathbb{D}(S), \circ)$. 
Proof. For $P, Q \in \mathbb{D}(\mathfrak{s})^{H}$ we have $P Q=P \times Q+$ lower order, hence $\widetilde{P Q}=$ $\widetilde{P} \circ \widetilde{Q}+$ lower order and the result follows by induction on the order.

\subsubsection{Example: the Laplace-Beltrami Operator}

Let $S=G / H$ be a pseudo-Riemannian symmetric space and $e(X, Y)$ an $e$ function of $S$. The space is equipped with the $G$-invariant metric defined by an $H$-invariant non-degenerate bilinear form on $\mathfrak{s}$; thus $\operatorname{det}_{\mathfrak{s}} \operatorname{Ad} h=1$ for all $h \in H$ since $H$ is connected. The next proposition relates the corresponding (pseudo-)Laplacian $\Delta \in \mathbb{D}(\mathfrak{s})^{H}$ on the tangent space, the Laplace-Beltrami operator $L \in \mathbb{D}(S)$ and the operators $\widetilde{\Delta} \in \mathbb{D}(S), \Delta_{e}\left(X, \partial_{X}\right)$ arising from $\Delta$ and $e$. As usual $J$ denotes the Jacobian of Exp.

Proposition 3.11 Let $S=G / H$ be a pseudo-Riemannian symmetric space $S=G / H$ with an e-function on $\Omega$ associated with $j=J^{1 / 2}$.

(i) Let $\varphi$ be a smooth function on an open subset of $S$. Then, for any $g H$ in this set, $L \varphi(g H)=\left.\Delta_{X} \varphi(g \cdot \operatorname{Exp} X)\right|_{X=0}$.

(ii) $\widetilde{\Delta}=L+\Delta J^{1 / 2}(0)$.

(iii) Assume $\partial_{Y} e(X, 0)=0$ identically. Then

$$
\begin{aligned}
& \Delta_{Y} e(X, 0)=\Delta J^{1 / 2}(0)-J(X)^{-1 / 2} \Delta\left(J(X)^{1 / 2}\right) \\
& \Delta_{e}\left(X, \partial_{X}\right)=\Delta_{X}+\Delta_{Y} e(X, 0) .
\end{aligned}
$$

If $u$ is an $H$-invariant distribution on $\Omega_{1}$,

$$
L \widetilde{u}=\left(\Delta u-J^{-1 / 2} \Delta\left(J^{1 / 2}\right) u\right)^{\sim} \text { on } \operatorname{Exp} \Omega_{1} .
$$

(iv) Let $G$ be semi-simple. For the pseudo-Riemannian structure defined on $G / H$ by the Killing form one has $\widetilde{\Delta}=L+(n / 12)$ with $n=\operatorname{dim} G / H$.

Remarks. (a) The assumption $\partial_{Y} e(X, 0)=0$ is satisfied by the rank one $e$ function of Section 3.7 (Proposition 3.24) and by the general $\left(J^{1 / 2}, \Phi\right)$-function of Chapter 4 (see Remark (d) after Theorem 4.20, with $\operatorname{det}_{\mathfrak{s}} \operatorname{Ad} h=1$ for all $h \in H$ implying $\operatorname{tr}_{\mathfrak{h}}$ ad $\mathfrak{h}_{*}=0$ by Lemma 4.11).

(b) The expression (iii) of $L \widetilde{u}$ extends Theorem 3.15 in [28] p. 273, proved by Helgason for Riemannian symmetric spaces of the noncompact type.

Proof. (i) Both sides are $G$-invariant operators on $S$ and it suffices to prove their equality at the origin, that is $g=e$. But, when using normal coordinates (the diffeomorphism Exp) in a neighborhood of the origin of $S$, the classical expression $\sum_{i, j} g^{i j}\left(\partial_{i} \partial_{j}-\Gamma_{i j}^{k} \partial_{k}\right)$ of $L([28]$ p. 247) reduces to $\Delta$ at the origin since, at this point, $\left(g^{i j}\right)$ is the identity matrix and the Christoffel symbols $\Gamma_{i j}^{k}$ vanish.

(ii) By (3.3) and (i),

$$
\widetilde{\Delta} \varphi(g H)=\left.\Delta_{X}\left(J(X)^{1 / 2} \varphi(g \cdot \operatorname{Exp} X)\right)\right|_{X=0}=\left(L+\Delta J^{1 / 2}(0)\right) \varphi(g H),
$$


since $J(0)=1$ and $\partial_{X} J^{1 / 2}(0)=0$.

(iii) The definition of $\Delta_{e}$ is

$\Delta_{e}\left(X, \partial_{X}\right) f(X)=\left.\Delta_{Y}(e(X, Y) f(X+Y))\right|_{Y=0}=\Delta f(X)+\Delta_{Y} e(X, 0) f(X)$

since $\partial_{Y} e(X, 0)=0$ and $e(X, 0)=1$ by Proposition 3.4 (ii). Applying Theorem $3.8(i)$ to an $H$-invariant distribution $u$ we have ${ }^{t} \widetilde{\Delta} \widetilde{u}=\widetilde{{ }^{t} \Delta_{e} u}$. But $\Delta$ and $L$ are symmetric operators, as well as $\Delta_{e}$ and $\widetilde{\Delta}$ computed above, therefore, by (ii),

$$
L \widetilde{u}=\left(\Delta u+\left(\Delta_{Y} e(X, 0)-\Delta J^{1 / 2}(0)\right) u\right) .
$$

The distribution $u=J(X)^{1 / 2} d X$ is $H$-invariant since $\operatorname{det}_{\mathfrak{s}} \operatorname{Ad} h=1$. Then $\widetilde{u}=d x$ (Example 1 in 3.1.1), $L \widetilde{u}=0$, whence

$$
\Delta\left(J(X)^{1 / 2}\right)+\left(\Delta_{Y} e(X, 0)-\Delta J^{1 / 2}(0)\right) J(X)^{1 / 2}=0
$$

and our claims follow.

(iv) The Taylor expansion at the origin of $J^{1 / 2}$ is

$$
\begin{aligned}
J(X)^{1 / 2} & =\left(\operatorname{det}_{\mathfrak{s}} \frac{\operatorname{sh}(\operatorname{ad} X)}{\operatorname{ad} X}\right)^{1 / 2}=\operatorname{det}_{\mathfrak{s}}\left(1+\frac{1}{6}(\operatorname{ad} X)^{2}+O\left(X^{4}\right)\right)^{1 / 2} \\
& =1+\frac{1}{12} \operatorname{tr}_{\mathfrak{s}}(\operatorname{ad} X)^{2}+O\left(X^{4}\right) .
\end{aligned}
$$

For $X \in \mathfrak{s}$ the Killing form of $\mathfrak{g}$ is

$$
B(X, X)=\operatorname{tr}_{\mathfrak{g}}(\operatorname{ad} X)^{2}=\operatorname{tr}_{\mathfrak{h}}(\operatorname{ad} X)^{2}+\operatorname{tr}_{\mathfrak{s}}(\operatorname{ad} X)^{2}=2 \operatorname{tr}_{\mathfrak{s}}(\operatorname{ad} X)^{2}
$$

(see (4.3) in Section 4.2.1). If $\Delta$ is the Laplacian associated to $B$ we get $\Delta B=2 n, \Delta J^{1 / 2}(0)=n / 12$ and the result follows from (ii).

Example. Let us define the $n$-dimensional real hyperbolic space $H^{n}(\mathbb{R})$ as the upper half-hyperboloid

$$
x_{0}^{2}-x_{1}^{2}-\cdots-x_{n}^{2}=1, x_{0}>0
$$

in $\mathbb{R}^{n+1}$, with origin $o=(1,0, \ldots, 0)$ and (Riemannian) metric induced by the Lorentzian metric

$$
-d x_{0}^{2}+d x_{1}^{2}+\cdots+d x_{n}^{2} .
$$

As usual $S^{n-1}$ denotes the unit sphere in $\mathbb{R}^{n}$. The map

$$
(r, \sigma) \longmapsto x=\left(x_{0}, x_{1}, \ldots, x_{n}\right)=(\operatorname{ch} r,(\operatorname{sh} r) \sigma)
$$

is a diffeomorphism of $] 0, \infty\left[\times S^{n-1}\right.$ onto $H^{n}(\mathbb{R}) \backslash\{o\}$ transforming the hyperbolic metric into

$$
d s^{2}=d r^{2}+(\operatorname{sh} r)^{2} d \sigma^{2},
$$


where $d \sigma^{2}$ is the canonical metric of $S^{n-1}$.

The length of a curve defined by differentiable functions $r(t), \sigma(t)$ with $0 \leq$ $t \leq T, r(0)=0, r(T)=R$ is

$$
\int_{0}^{T} \sqrt{r^{\prime}(t)^{2}+(\operatorname{sh} r(t))^{2}\left\|\sigma^{\prime}(t)\right\|^{2}} d t \geq \int_{0}^{T} r^{\prime}(t) d t=R,
$$

where $\|$.$\| is the Euclidean norm in \mathbb{R}^{n}$. Taking $r(t)=t$ and $\sigma(t)=\sigma$ (constant) we obtain a curve with minimal length (equal to $R$ ) among all curves from the origin to the point $(\operatorname{ch} R,(\operatorname{sh} R) \sigma)$ in $H^{n}(\mathbb{R})$. This curve also has unit speed parametrization; it is therefore the geodesic between those points, defined by $x(t)=(\operatorname{ch} t,(\operatorname{sh} t) \sigma)$. The initial speed is $V=x^{\prime}(0)=$ $(0, \sigma)$, hence $x(t)=\operatorname{Exp} t V$. Replacing $V$ by $r V$ we see that $\operatorname{Exp}$ is simply the map $(r, \sigma) \mapsto(r, \sigma)$ if we use Euclidean polar coordinates in the tangent space and geodesic polar coordinates in $H^{n}(\mathbb{R})$.

The metric (3.14) gives the hyperbolic volume element $d v=(\operatorname{sh} r)^{n-1} d r d \Sigma$ where $d \Sigma$ is the volume element of $S^{n-1}$. Let $\omega_{n-1}=\int_{S^{n-1}} d \Sigma$. For any radial (continuous) function $f=f(r)$ on $H^{n}(\mathbb{R})$ we have

$$
\int_{H^{n}(\mathbb{R})} f d v=\omega_{n-1} \int_{0}^{\infty} f(r)(\operatorname{sh} r)^{n-1} d r .
$$

When transferred to the tangent space via Exp (with Jacobian $J(X)=J(r)$ ) this integral is also

$$
\int_{\mathbb{R}^{n}} f(\operatorname{Exp} X) J(X) d X=\omega_{n-1} \int_{0}^{\infty} f(r) J(r) r^{n-1} d r,
$$

whence $J(X)=(\operatorname{sh} r / r)^{n-1}$ for $X=r \sigma$.

The metric (3.14) also gives the Laplace-Beltrami operator of $H^{n}(\mathbb{R})$ in geodesic polar coordinates (cf. [28] p. 313):

$$
L=\partial_{r}^{2}+(n-1) \operatorname{coth} r \partial_{r}+L_{r}
$$

where $L_{r}$ is the Laplace-Beltrami operator on the sphere with center $o$ and radius $r$ in the hyperbolic space. Similarly

$$
\Delta=\partial_{r}^{2}+\frac{n-1}{r} \partial_{r}+\Delta_{r}
$$

in Euclidean polar coordinates of $\mathbb{R}^{n}$. The «spherical» operators $L_{r}$ and $\Delta_{r}$ kill radial functions.

Our transfer map for functions is given by $\widetilde{u}(\operatorname{Exp} X)=J(X)^{-1 / 2} u(X)$ that is, using the above coordinates on $H^{n}(\mathbb{R})$ and its tangent space,

$$
\widetilde{u}(r, \sigma)=\left(\frac{\operatorname{sh} r}{r}\right)^{(1-n) / 2} u(r, \sigma) .
$$


Restricting ourselves to radial functions we can then compare the radial parts of $L$ and $\Delta$ above as in Helgason's proof of his theorem in [28] p. 273. An easy calculation leads to

$$
L \widetilde{u}=(\Delta u+f u)^{\sim}
$$

with

$$
f:=-J^{-1 / 2} \Delta J^{1 / 2}=\frac{(n-1)(n-3)}{4}\left(\frac{1}{r^{2}}-\frac{1}{\operatorname{sh}^{2} r}\right)-\left(\frac{n-1}{2}\right)^{2} .
$$

Thus ${ }^{2} \Delta J^{1 / 2}(0)=-f(0)=n(n-1) / 6$ and, comparing with (ii) and (iii) in the proposition, it follows that

$$
\Delta_{Y} e(X, 0)=\frac{(n-1)(n-3)}{4}\left(\frac{1}{r^{2}}-\frac{1}{\operatorname{sh}^{2} r}-\frac{1}{3}\right)
$$

with $r=\|X\|$, if $e$ is an $e$-function of $H^{n}(\mathbb{R})$ associated with $J^{1 / 2}$.

\subsubsection{Towards a Generalized Duflo Isomorphism}

With the above tools at hand we can now try to extend to general symmetric spaces Duflo's isomorphism for Lie groups (Section 1.1). By Theorem 3.9 the transfer map $\sim$ is an isomorphism of $\left(S(\mathfrak{s})^{H}, \times\right)$ onto $(\mathbb{D}(S), \circ)$, and the problem is to construct an isomorphism $\varepsilon:\left(S(\mathfrak{s})^{H}, \cdot\right) \rightarrow\left(S(\mathfrak{s})^{H}, \times\right)$ between the classical product $(\cdot)$ and the product $(\times)$ in the symmetric algebra. The map $\gamma:\left(S(\mathfrak{s})^{H}, \cdot\right) \rightarrow(\mathbb{D}(S), \circ)$ defined by $\gamma(P):=\widetilde{\varepsilon(P)}$ will then be an isomorphism of algebras, the «generalized Duflo isomorphism».

The commutativity of the algebra $\mathbb{D}(S)$ (or of the product $\times$ ) is of course a necessary condition for the existence of $\gamma$. This property has been established by several authors under various assumptions (see the Notes of this chapter). It will follow here from the symmetry of an $e$-function (Theorem 4.24).

Example 1. If $S$ is a special symmetric space the products $(\times)$ and $(\cdot)$ coincide and we may take $\varepsilon(P)=P, \gamma(P)=\widetilde{P}$.

Example 2. Assume $\mathbb{D}(S)$ is commutative and $\left(S(\mathfrak{s})^{H}, \cdot\right)$ is a polynomial algebra with generators $P_{1}, \ldots, P_{l}$ (as holds true for all Riemannian symmetric spaces of the noncompact type). Then there exists a unique isomorphism $\varepsilon$ : $\left(S(\mathfrak{s})^{H}, \cdot\right) \rightarrow\left(S(\mathfrak{s})^{H}, \times\right)$ such that $\varepsilon\left(P_{j}\right)=P_{j}$ for $j=1, \ldots, l$, and $\gamma(P)=$ $\widetilde{\varepsilon(P)}$ is a degree preserving generalized Duflo isomorphism (characterized by $\gamma\left(P_{j}\right)=\widetilde{P}_{j}$ for all $\left.j\right)$.

\footnotetext{
${ }^{2}$ This does not contradict (iv) in the proposition: we are not using here the metric defined by the Killing form.
} 
Indeed any $P \in S(\mathfrak{s})^{H}$ can be uniquely written as

$$
P=\sum_{\alpha \in \mathbb{N}^{l}} a_{\alpha} P_{1}^{\alpha_{1}} \cdots P_{l}^{\alpha_{l}}
$$

(product $\cdot$ ), and the maps defined by

$$
\varepsilon(P):=\sum_{\alpha \in \mathbb{N}^{l}} a_{\alpha} P_{1}^{\times \alpha_{1}} \times \cdots \times P_{l}^{\times \alpha_{l}}, \gamma(P)=\sum_{\alpha \in \mathbb{N}^{l}} a_{\alpha}{\widetilde{P_{1}}}^{\alpha_{1}} \circ \cdots \circ \widetilde{P}_{l}^{\alpha_{l}}
$$

(product $\times$, resp. o) fulfill our requirements because of Corollary 3.10 and the commutativity of these products. Remembering (3.13) this $\varepsilon$ may also be written as

$$
\varepsilon(P)=\sum_{\alpha \in \mathbb{N}^{l}} a_{\alpha} A_{1}^{\alpha_{1}} \cdots A_{l}^{\alpha_{l}} 1
$$

where $A_{j}: S(\mathfrak{s})^{H} \rightarrow S(\mathfrak{s})^{H}$ is the operator defined by $A_{j} P=P_{j} \times P$, that is

$$
A_{j}:=\sum_{\beta, \gamma \in \mathbb{N}^{n}} \frac{1}{\beta ! \gamma !} \partial_{X}^{\beta} \partial_{Y}^{\gamma} e(0,0) \partial_{\xi}^{\beta} P_{j}(\xi) \partial_{\xi}^{\gamma} .
$$

Example 3. Assume one of the generators in Example 2, say $Q:=P_{1}$, is homogeneous of degree two, and the symmetric space is endowed with an $e$-function such that $e(X, Y)=e(Y, X)$ and $e(X, 0)=1, \partial_{Y} e(X, 0)=0$ (see Remark $(a)$ in Section 3.3.3). Setting $e_{Q}(X):=\left.Q\left(\partial_{Y}\right) e(X, Y)\right|_{Y=0}$, Leibniz' formula implies

$$
\begin{aligned}
Q_{e}\left(X, \partial_{X}\right) f(X) & =Q\left(\partial_{Y}\right)(e(X, Y) f(X+Y))_{Y=0} \\
& =\left(Q\left(\partial_{X}\right)+e_{Q}(X)\right) f(X) .
\end{aligned}
$$

Note that $e_{Q}(0)=0$. Thus $^{3} Q_{e}(X, \eta)=Q(\eta)+e_{Q}(X)$ and, by Theorem 3.9,

$$
(P \times Q)(\xi)=\left.\left(Q_{e}\left(\partial_{\xi}, \eta\right) P(\xi)\right)\right|_{\xi=\eta}=\left(Q(\xi)+e_{Q}\left(\partial_{\xi}\right)\right) P(\xi)
$$

for $P \in S(\mathfrak{s})^{H}, \xi \in \mathfrak{s}^{*}$. The required map $\varepsilon$ satisfies $\varepsilon(Q)=Q$ and $\varepsilon(P \cdot Q)=$ $\varepsilon(P) \times \varepsilon(Q)=Q \cdot \varepsilon(P)+e_{Q}(\partial) \varepsilon(P)$. In other words

$$
\varepsilon(Q)=Q \text { and }[\varepsilon, Q]=e_{Q}(\partial) \circ \varepsilon,
$$

where $[\varepsilon, Q]$ denotes the commutator of two linear endomorphisms of $S(\mathfrak{s})^{H}$, with $Q$ meaning here the mutliplication operator by $Q$ in $\left(S(\mathfrak{s})^{H}, \cdot\right)$.

If $l=1$, that is if $S(\mathfrak{s})^{H}$ is the algebra of all polynomials $p(Q), p \in \mathbb{C}[X]$, these conditions determine $\varepsilon$ :

$$
\varepsilon(p(Q))=p\left(Q+e_{Q}(\partial)\right) 1
$$

\footnotetext{
${ }^{3} \mathrm{~A}$ more explicit expression can be given when Proposition 3.11 (iii) applies.
} 
This special case of (3.15) follows from (3.16) applied to $P=Q^{\times k}$; here again $Q$ on the right-hand side is viewed as a multiplication operator.

It would be interesting to generalize these examples: can one construct an isomorphism $\varepsilon:\left(S(\mathfrak{s})^{H}, \cdot\right) \rightarrow\left(S(\mathfrak{s})^{H}, \times\right)$ by means of $e$, only assuming the symmetry $e(X, Y)=e(Y, X)$ ? See Section 3.6.3.b for a hint in this direction.

\section{$3.4 \quad e$-Functions, Mean Values and Spherical Functions}

Throughout this section $S=G / H$ is a symmetric space with $H$ compact. The space is now Riemannian, with the $G$-invariant metric defined by an $H$-invariant scalar product on $\mathfrak{s}$. We denote by $|X|$ the corresponding norm of $X \in \mathfrak{s}$ and by $|\xi|$ the dual norm of $\xi \in \mathfrak{s}^{*}$. Let $d h$ be the Haar measure of $H$ normalized by $\int_{H} d h=1$.

Recall that a spherical function of $S$ is an $H$-invariant eigenfunction $\varphi$ of all operators in $\mathbb{D}(S)$, normalized by $\varphi(o)=1$. Spherical functions are analytic on $S$ because of the ellipticity of the Laplace-Beltrami operator $L \in \mathbb{D}(S)$. Our next goal is to relate spherical functions and mean value operators to an $e$-function of $S$ by means of Taylor expansions. Let us begin with the case of an isotropic space, in order to motivate the more general construction in 3.4 .2 below.

\subsubsection{The Case of an Isotropic Riemannian Symmetric Space}

In this subsection we assume $H$ compact and $S=G / H$ isotropic, meaning that the adjoint action of $H$ is transitive on the unit sphere of the tangent space $\mathfrak{s}$ to $S$ at the origin. As is well-known (e.g. [61] p. 295), a connected Riemannian manifold is isotropic if and only if it is two point homogeneous (its isometry group is transitive on equidistant pairs of points), or else if and only if it is a Euclidean space or a symmetric space of rank one. Then $\mathbb{D}(\mathfrak{s})^{H}$, resp. $\mathbb{D}(S)$, is the algebra of all polynomials in the (Euclidean) Laplace operator $\Delta$ of $\mathfrak{s}$, resp. in the Laplace-Beltrami operator $L$ of $S$ ([28] p. 288). We also assume here $j=J^{1 / 2}$.

a. On the open neighborhood of the origin where we can use the Exp chart we may write $\varphi=\widetilde{\psi}$ that is $\psi(X)=J(X)^{1 / 2} \varphi(\operatorname{Exp} X)$. Then $L \varphi=$ $\left(\widetilde{\Delta}-\Delta J^{1 / 2}(0)\right) \widetilde{\psi}$ by Proposition 3.11 and $\varphi$ is spherical if and only if $\psi$ is $H$-invariant, $\psi(0)=1$ and $\widetilde{\Delta} \widetilde{\psi}=\lambda \widetilde{\psi}$ for some constant $\lambda$, or else $\Delta_{e} \psi=\lambda \psi$ by Theorem 3.8 (ii). Picking an $e$-function of $S$ such that $\partial_{Y} e(X, 0)=0$ we obtain the equation

$$
\Delta \psi(X)=\left(\lambda-\Delta_{Y} e(X, 0)\right) \psi(X), \psi(0)=1
$$


by Proposition 3.11 (iii). Equation (3.17) is a perturbation of $\Delta \psi=\lambda \psi$, its analog for a special isotropic symmetric space. Since $\Delta$ is an elliptic operator its solutions are analytic. In view of the $H$-invariance the Taylor series at the origin have the following form

$$
\psi(X)=\sum_{m=0}^{\infty} a_{m}(\lambda) \frac{|X|^{2 m}}{c_{m}}, \Delta_{Y} e(X, 0)=\sum_{m=1}^{\infty} e_{m} \frac{|X|^{2 m}}{c_{m}},
$$

where $a_{m}(\lambda)$ (resp. $e_{m}$ ) are unknown (resp. known) coefficients and the constants $c_{m}:=\left\langle\left.\Delta^{m}|| X\right|^{2 m}\right\rangle=\Delta^{m}|X|^{2 m}$, introduced here for convenience, are

$$
c_{0}=1, c_{m}=2^{m} m ! n(n+2) \cdots(n+2 m-2) \text { with } m \geq 1, n=\operatorname{dim} S .
$$

Thus $a_{m}(\lambda)=\Delta^{m} \psi(0), e_{m}=\Delta_{X}^{m} \Delta_{Y} e(0,0)$ and (3.17) is equivalent to

$$
\begin{aligned}
a_{0}(\lambda) & =1, a_{1}(\lambda)=\lambda=\Delta \psi(0) \\
a_{m+1}(\lambda) & =\lambda a_{m}(\lambda)-\sum_{l=0}^{m-1} \frac{c_{m}}{c_{m-l} c_{l}} e_{m-l} a_{l}(\lambda), m \geq 1 .
\end{aligned}
$$

Solving this inductively we obtain

$$
\begin{aligned}
\psi(X) & =J(X)^{1 / 2} \varphi(\operatorname{Exp} X) \\
& =1+\lambda \frac{|X|^{2}}{c_{1}}+\left(\lambda^{2}-e_{1}\right) \frac{|X|^{4}}{c_{2}}+\left(\lambda^{3}-\left(3+\frac{4}{n}\right) e_{1} \lambda-e_{2}\right) \frac{|X|^{6}}{c_{3}}+\cdots
\end{aligned}
$$

a convergent series in a neighborhood of the origin. Indeed the convergence of the power series $\sum e_{m}|X|^{2 m} / c_{m}$ implies $\left|e_{m}\right| / c_{m} \leq C R^{m}$ for some positive constants $C, R$ and $\left|a_{m}(\lambda)\right| / c_{m} \leq C^{\prime} R^{m}$ easily follows by induction, with $C^{\prime}>0$ depending on $\lambda$.

In the case of a special isotropic space $(e(X, Y)=1)$ we obtain $a_{m}(\lambda)=$ $\lambda^{m}$ and formula (3.21) boils down to the classical expansion of modified Bessel functions

$$
\psi(X)=\sum_{m=0}^{\infty} \lambda^{m} \frac{|X|^{2 m}}{c_{m}}=\Gamma(n / 2)\left(\frac{2}{|X| \sqrt{-\lambda}}\right)^{(n / 2)-1} J_{(n / 2)-1}(|X| \sqrt{-\lambda}) .
$$

b. More generally, a similar expansion can be given for the mean value operator

$M^{X} \varphi(g H):=\int_{H} \varphi(g h \cdot \operatorname{Exp} X) d h=\int_{H} \varphi(g \cdot \operatorname{Exp}(h \cdot X)) d h, X \in \mathfrak{s}, g \in G$,

applied to an arbitrary analytic function $\varphi$ on the symmetric space $S$. In an isotropic space $H$-orbits are spheres and the above integral is the average of $\varphi$ over the sphere with center $g H$ and radius $|X|$. 
For fixed $g, J(X)^{1 / 2} M^{X} \varphi(g H)$ is an $H$-invariant analytic function of $X$ and can be expanded as

$$
J(X)^{1 / 2} M^{X} \varphi(g H)=\sum_{m=0}^{\infty} \varphi_{m}(g H) \frac{|X|^{2 m}}{c_{m}},
$$

where the coefficients $\varphi_{m}$ are given by

$$
\begin{aligned}
\varphi_{m}(g H) & =\left.\Delta_{X}^{m}\left(J(X)^{1 / 2} M^{X} \varphi(g H)\right)\right|_{X=0} \\
& =\left.\Delta_{X}^{m}\left(J(X)^{1 / 2} \varphi(g \cdot \operatorname{Exp} X)\right)\right|_{X=0}=\widetilde{\Delta^{m}} \varphi(g H)
\end{aligned}
$$

in view of the $H$-invariance of $\Delta$ and the definition (3.3). Thus

$$
J(X)^{1 / 2} M^{X} \varphi(g H)=\sum_{m=0}^{\infty} \widetilde{\Delta^{m}} \varphi(g H) \frac{|X|^{2 m}}{c_{m}} .
$$

The operator $\widetilde{\Delta^{m}}$ belongs to $\mathbb{D}(S)$ and can therefore be expressed as a polynomial in $\widetilde{\Delta}$; this will allow linking our expansion of $M^{X}$ with the above expansion of a spherical function satisfying $\widetilde{\Delta} \varphi=\lambda \varphi$. The details can be carried out inductively as follows.

Applying to $\Delta^{m}(\xi)=|\xi|^{2 m}$ and $\Delta$ the product $\times$ of Theorem 3.9 we have

$$
\left(\Delta^{m} \times \Delta\right)(\xi)=\Delta_{e}\left(\partial_{\xi}, \eta\right)\left(|\xi|^{2 m}\right)_{\xi=\eta}
$$

and, by Proposition 3.11 (iii) and (3.18),

$$
\Delta_{e}(X, \eta)=|\eta|^{2}+\Delta_{Y} e(X, 0)=|\eta|^{2}+\sum_{l=1}^{\infty} \frac{e_{l}}{c_{l}}|X|^{2 l}
$$

whence $\Delta_{e}\left(\partial_{\xi}, \eta\right)=|\eta|^{2}+\sum_{l=1}^{\infty}\left(e_{l} / c_{l}\right) \Delta_{\xi}^{l}$.

Since $\Delta_{\xi}^{l}\left(|\xi|^{2 m} / c_{m}\right)=|\xi|^{2(m-l)} / c_{m-l}$ we obtain

$$
\left(\Delta^{m} \times \Delta\right)(\xi)=\Delta^{m+1}(\xi)+c_{m} \sum_{l=1}^{m} \frac{e_{l}}{c_{l}} \frac{\Delta^{m-l}(\xi)}{c_{m-l}} .
$$

We now apply $\sim$ to both sides, hence the following analog of (3.20)

$$
\widetilde{\Delta^{m+1}}=\widetilde{\Delta^{m}} \circ \widetilde{\Delta}-\sum_{l=0}^{m-1} \frac{c_{m}}{c_{m-l} c_{l}} e_{m-l} \widetilde{\Delta^{l}}
$$

implying that $\widetilde{\Delta^{m}}=a_{m}(\widetilde{\Delta})$ where $a_{m}$ is a polynomial of degree $m$ inductively defined by this equation and the coefficients $e_{l}$, clearly the same polynomial as before. We finally obtain

$$
J(X)^{1 / 2} M^{X} \varphi(g H)=\sum_{m=0}^{\infty}\left(a_{m}(\widetilde{\Delta}) \varphi\right)(g H) \frac{|X|^{2 m}}{c_{m}}
$$


for any analytic function $\varphi$ on $S$. If $\varphi$ is spherical, $\widetilde{\Delta} \varphi=\lambda \varphi$, we have $M^{X} \varphi(g H)=\varphi(\operatorname{Exp} X) \varphi(g H)$ by the classical functional equation and this of course gives (3.21) again.

Remark. In [24] Gray and Willmore proved the following mean value expansion for an arbitrary $n$-dimensional Riemannian manifold $M$. Let $u$ be an analytic function on a neighborhood of a point $a \in M$ and let $M^{r} u(a)$ denote the mean value of $u$ over the (Riemannian) sphere $S(a, r)$ with center $a$ and radius $r$. Then

$$
M^{r} u(a)=\frac{f_{n}\left(r \sqrt{-\Delta_{a}}\right)\left(J_{a} u\right)(a)}{f_{n}\left(r \sqrt{-\Delta_{a}}\right)\left(J_{a}\right)(a)},
$$

where $f_{n}$ is the modified Bessel function

$$
f_{n}(x)=\sum_{m=0}^{\infty}(-1)^{m} \frac{x^{2 m}}{c_{m}}
$$

(with $c_{m}$ as in (3.19)), $J_{a}$ is the Jacobian of the exponential map $\operatorname{Exp}_{a}$ at $a$, $\Delta_{a}$ is the differential operator on $M$ defined by $\left(\Delta_{a} u\right) \circ \operatorname{Exp}_{a}=\Delta\left(u \circ \operatorname{Exp}_{a}\right)$ and $\Delta$ is, as above, the Euclidean Laplace operator on the tangent space to $M$ at $a$. This generalized Pizzetti formula follows easily from the corresponding formula in Euclidean space, since the Riemannian sphere $S(a, r)$ is the image under $\operatorname{Exp}_{a}$ of the Euclidean sphere with center 0 and radius $r$. For isotropic Riemannian symmetric spaces $M=G / H$ the spheres are $H$-orbits and we may compare (3.25) with our (3.24): the Gray-Willmore expansion uses the same simple power series as in the Euclidean case, but in general the differential operator $\Delta_{a}$ is not $G$-invariant on $M$. Thus no simple expansion of the spherical functions seems to come out of it.

\subsubsection{Expansion of Mean Value Operators and Spherical Functions}

Going back to a general Riemannian symmetric space $S=G / H$ we shall now extend the results proved in the isotropic case. The compactness of $H$ implies that the algebra $S(\mathfrak{s})^{H}=\mathbb{D}(\mathfrak{s})^{H}$ is finitely generated ([28] p. 352). Let $P_{1}, \ldots, P_{l}$ be a system of generators, homogeneous with respective degrees $d_{1}, \ldots, d_{l}$. They may be algebraically dependent, but from the set of all

$$
P^{\alpha}:=P_{1}^{\alpha_{1}} \cdots P_{l}^{\alpha_{l}}, \alpha \in \mathbb{N}^{l},
$$

we can extract a basis of $\mathbb{D}(\mathfrak{s})^{H}$ as a vector space. Let $B \subset \mathbb{N}^{l}$ denote the corresponding subset of indices.

To introduce the dual basis we note that the Fischer product allows to identify $S\left(\mathfrak{s}^{*}\right)^{H}$ with the space of finite linear forms on $S(\mathfrak{s})^{H}$ (see 3.3.1). Indeed let $\pi: S(\mathfrak{s}) \rightarrow S(\mathfrak{s})^{H}$ be the projection given by $H$-invariant mean. If 
$\ell$ is a finite linear form on $S(\mathfrak{s})^{H}$, then $\ell \circ \pi$ is a finite linear form on $S(\mathfrak{s})$ and there exists a unique $Q \in S\left(\mathfrak{s}^{*}\right)$ such that $\ell \circ \pi(P)=\langle P \mid Q\rangle$ for all $P \in S(\mathfrak{s})$. Replacing $P$ by $h \cdot P$ with $h \in H$ we obtain $\langle P \mid Q\rangle=\langle h \cdot P \mid Q\rangle=\left\langle P \mid h^{-1} \cdot Q\right\rangle$, hence $Q \in S\left(\mathfrak{s}^{*}\right)^{H}$ by uniqueness, as claimed.

The conditions $\ell_{\alpha}\left(P^{\beta}\right)=\delta_{\alpha \beta}$ (Kronecker symbol) for $\alpha, \beta \in B$ define forms $\ell_{\alpha}$ which make up a basis of the space of finite linear forms on $S(\mathfrak{s})^{H}$. Denoting by $P_{\alpha}^{*}$ the corresponding elements of $S\left(\mathfrak{s}^{*}\right)^{H}$ we have

$$
\left\langle P^{\beta} \mid P_{\alpha}^{*}\right\rangle=\delta_{\alpha \beta} \text { for } \alpha, \beta \in B
$$

and the decomposition of any $Q \in S\left(\mathfrak{s}^{*}\right)^{H}$ is $Q=\sum_{\alpha \in B}\left\langle P^{\alpha} \mid Q\right\rangle P_{\alpha}^{*}$.

Each $P^{\alpha}$ is homogeneous of degree $\alpha \cdot d=\alpha_{1} d_{1}+\cdots+\alpha_{l} d_{l}$ and the same holds for $P_{*}^{\alpha}$ : indeed for all $\alpha, \beta \in B$ and $t \in \mathbb{R}$ we have

$$
\begin{aligned}
\left\langle P^{\beta}\left(\partial_{X}\right) \mid P_{\alpha}^{*}(t X)\right\rangle & =t^{\beta \cdot d} P^{\beta}(\partial) P_{\alpha}^{*}(0)=t^{\beta \cdot d} \delta_{\alpha \beta} \\
& =t^{\alpha \cdot d} \delta_{\alpha \beta}=t^{\alpha \cdot d}\left\langle P^{\beta}\left(\partial_{X}\right) \mid P_{\alpha}^{*}(X)\right\rangle,
\end{aligned}
$$

hence $P_{\alpha}^{*}(t X)=t^{\alpha \cdot d} P_{\alpha}^{*}(X)$.

Example 1 (trivial case): $H=\{e\}, P^{\alpha}=\partial_{X}^{\alpha}, P_{\alpha}^{*}=X^{\alpha} / \alpha !$.

Example 2 (isotropic case): $P^{\alpha}=\Delta^{m}, P_{\alpha}^{*}=|X|^{2 m} / c_{m}$ with $\alpha=m \in B=$ $\mathbb{N}$ and $c_{m}$ defined by (3.19).

Lemma 3.12 ( $H$ compact) Any $H$-invariant function $f$, analytic in a neighborhood of 0 in $\mathfrak{s}$, admits a unique $H$-invariant Taylor expansion

$$
f(X)=\sum_{\alpha \in B} a_{\alpha} P_{\alpha}^{*}(X),
$$

with coefficients given by $a_{\alpha}=P^{\alpha}\left(\partial_{X}\right) f(0)=\left\langle P^{\alpha} \mid f\right\rangle$. The series converges in the following sense: there exists $R>0$ such that

$$
\sum_{q \geq 0}\left|\sum_{\alpha \cdot d=q} a_{\alpha} P_{\alpha}^{*}(X)\right|<\infty \text { for }|X|<R .
$$

This expansion of $f$ can be differentiated term by term for $|X|<R$.

Proof. For some $R>0$ the classical Taylor series $f(X)=\sum_{\sigma \in \mathbb{N}^{n}} f_{\sigma} X^{\sigma}$ converges absolutely and can be differentiated term by term for $|X|<R$. Gathering terms of equal degrees, the same properties a fortiori hold for $\sum_{q \geq 0}\left(\sum_{|\sigma|=q} f_{\sigma} X^{\sigma}\right)$. Each term of this new series, as an invariant $q$ homogeneous polynomial in $X$, is a linear combination of the $P_{\alpha}^{*}$ 's with $\alpha \cdot d=q$, hence the expansion of $f$. The formula for $a_{\alpha}$ comes out when applying $P^{\alpha}\left(\partial_{X}\right)$ term by term. 
Example (generalized Bessel functions). For $\xi \in \mathfrak{s}^{*}$ and $X \in \mathfrak{s}$ let

$$
\varphi_{\xi}^{0}(X):=\int_{H} e^{<\xi, h \cdot X>} d h .
$$

Then $P\left(\partial_{X}\right) \varphi_{\xi}^{0}(X)=P(\xi) \varphi_{\xi}^{0}(X)$ for any $P \in \mathbb{D}(\mathfrak{s})^{H}$ and $\varphi_{\xi}^{0}$ is a spherical function for the flat space $\mathfrak{s}$. Lemma 3.12 gives the expansion

$$
\varphi_{\xi}^{0}(X)=\int_{H} e^{<\xi, h \cdot X>} d h=\sum_{\alpha \in B} P^{\alpha}(\xi) P_{\alpha}^{*}(X),
$$

a convergent series in the above sense for all $X$ and $\xi$.

Let us take up the mean value operator again

$$
M^{X} \varphi(g H):=\int_{H} \varphi(g \cdot \operatorname{Exp}(h \cdot X)) d h, X \in \mathfrak{s}, g \in G .
$$

This integral over $H$ is an average of $\varphi$ over a submanifold of the sphere with center $g H$ and radius $|X|$. Let $g$ be fixed and $\varphi$ be analytic in a neighborhood of $g H$. According to the previous lemma the $H$-invariant function $j(X) M^{X} \varphi(g H)$ of $X$ can be expanded as

$$
j(X) M^{X} \varphi(g H)=\sum_{\alpha \in B} a_{\alpha}(g H) P_{\alpha}^{*}(X)
$$

with the coefficients

$a_{\alpha}(g H)=P^{\alpha}\left(\partial_{X}\right)\left(j(X) M^{X} \varphi(g H)\right)_{X=0}=P^{\alpha}\left(\partial_{X}\right)(j(X) \varphi(g \cdot \operatorname{Exp} X))_{X=0}$, hence the following generalization of (3.22)

$$
j(X) M^{X} \varphi(g H)=\sum_{\alpha \in B} \widetilde{P^{\alpha}} \varphi(g H) P_{\alpha}^{*}(X) .
$$

We now give another version of this expansion in terms of the generators $\widetilde{P_{1}}, \ldots, \widetilde{P}_{l}$ of the algebra $\mathbb{D}(S)$ (see Corollary 3.10 ). Let us recall that this algebra is commutative ([28] p. 293). Given an $e$-function we shall write

$$
e(X, Y)=\sum_{\sigma, \tau \in \mathbb{N}^{n}} e_{\sigma \tau} X^{\sigma} Y^{\tau} \text { with } e_{\sigma \tau}=\frac{1}{\sigma ! \tau !} \partial_{X}^{\sigma} \partial_{Y}^{\tau} e(0,0)
$$

its Taylor expansion at the origin with respect to some basis of $\mathfrak{s}$. In view of Proposition 3.4 (ii) we have

$$
e_{00}=1, e_{\sigma 0}=e_{0 \tau}=0
$$

for all $\sigma, \tau \neq 0$. Although unessential here we note that for the CampbellHausdorff $e$-function of chapter 4 with $j=J^{1 / 2}$ we also have $e_{\sigma \tau}=0$ for $1 \leq|\sigma|+|\tau| \leq 3$, as follows from Proposition 4.31 and Lemma 4.11. Formula (3.24) extends as follows. 
Theorem 3.13 ( $H$ compact) Let $S=G / H$ be a Riemannian symmetric space with an e-function, and $P_{1}, \ldots, P_{l}$ a given system of generators of the algebra $\mathbb{D}(\mathfrak{s})^{H}$. Let $\varphi$ be an analytic function on a neighborhood of a point $g_{0} H$ in $S$. Then there exist a neighborhood $V$ of $g_{0}$ in $G$, a radius $R>0$ and a sequence of polynomials $\left(A_{\alpha}\right)_{\alpha \in B}$ such that for $X \in \mathfrak{s},|X|<R$ and $g \in V$

$$
j(X) M^{X} \varphi(g H)=\sum_{\alpha \in B} A_{\alpha}\left(e ; \widetilde{P}_{1}, \ldots, \widetilde{P}_{l}\right) \varphi(g H) P_{\alpha}^{*}(X),
$$

with convergence in the sense of Lemma 3.12.

Each $A_{\alpha}\left(e ; \lambda_{1}, \ldots, \lambda_{l}\right)$ is a polynomial in the $\lambda_{j}($ with $1 \leq j \leq l)$ and the $e_{\sigma \tau}$ (with $|\sigma|+|\tau| \leq \alpha \cdot d$ ), homogeneous of degree $\alpha \cdot d$ if one assigns $\operatorname{deg} \lambda_{j}=$ $\operatorname{deg} P_{j}=d_{j}$ and $\operatorname{deg} e_{\sigma \tau}=|\sigma|+|\tau|$. Furthermore

$$
A_{\alpha}\left(e ; \lambda_{1}, \ldots, \lambda_{l}\right)=\lambda_{1}^{\alpha_{1}} \cdots \lambda_{l}^{\alpha_{l}}+\text { lower degree in } \lambda_{1}, \ldots, \lambda_{l} .
$$

The coefficients of $A_{\alpha}$ only depend on the structure of the algebra $\mathbb{D}(\mathfrak{s})^{H}$ and the choice of generators $P_{j}$.

This theorem provides a more precise form, for Riemannian symmetric spaces, of a result proved by Helgason for general homogeneous Riemannian spaces $G / H([29]$ p. 77$)$.

A spherical function $\varphi$ satisfies the functional equation $M^{X} \varphi(g H)=$ $\varphi(\operatorname{Exp} X) \varphi(g H)$ and we immediately infer the following expansion of $\varphi$.

Corollary 3.14 With the notation and assumptions of Theorem 3.13, let $\varphi$ be a spherical function on $S$ and $\lambda_{j}$ the corresponding eigenvalues: $\widetilde{P}_{j} \varphi=\lambda_{j} \varphi$ for $j=1, \ldots, l$. Then there exists $R>0$ such that for $X \in \mathfrak{s}$ and $|X|<R$

$$
j(X) \varphi(\operatorname{Exp} X)=\sum_{\alpha \in B} A_{\alpha}\left(e ; \lambda_{1}, \ldots, \lambda_{l}\right) P_{\alpha}^{*}(X) .
$$

Proof of Theorem 3.13. The function $(g, X) \mapsto \varphi(g \cdot \operatorname{Exp} X)$ is analytic in a neighborhood of $\left(g_{0}, 0\right)$, hence there exist a neighborhood $V$ of $g_{0}$ and $R>0$ such that it has a power series expansion (by means of local coordinates) on $V \times\{|X|<R\}$, and the same holds true for $j(X) M^{X} \varphi(g H)$.

In view of (3.27) it will suffice to prove that

$$
\widetilde{P^{\alpha}}=A_{\alpha}\left(e ; \widetilde{P_{1}}, \ldots, \widetilde{P}_{l}\right)
$$

for any $\alpha \in \mathbb{N}^{l}$, which can be obtained by induction on $\alpha \cdot d$.

First $\widetilde{P^{0}}=1$ and the claim follows with $A_{0}=1$. Then, if $\alpha \in \mathbb{N}^{l}$ and $\alpha_{j} \geq 1$ for some $j$ we may write $P^{\alpha}=P^{\beta} P_{j}$ (usual product in $\mathbb{D}(\mathfrak{s})^{H}$ ) with $\beta \in \mathbb{N}^{l}$. Using the $\times$ product (3.13) we have

$$
P^{\beta} \times P_{j}=P^{\beta} P_{j}+Q \text { with } Q=\sum_{|\sigma| \geq 1,|\tau| \geq 1} e_{\sigma \tau}\left(\partial^{\sigma} P^{\beta}\right)\left(\partial^{\tau} P_{j}\right) \in \mathbb{D}(\mathfrak{s})^{H},
$$


therefore $\widetilde{P^{\alpha}}=\widetilde{P^{\beta}} \circ \widetilde{P_{j}}-\widetilde{Q}$ by Theorem 3.9. But $Q \in \mathbb{D}(\mathfrak{s})^{H}$ decomposes as $Q=\sum_{\gamma \in B} c_{\gamma} P^{\gamma}$ with $c_{\gamma}=\left\langle Q \mid P_{\gamma}^{*}\right\rangle$ (Fischer product), thus

$$
\widetilde{P^{\alpha}}=\widetilde{P^{\beta}} \circ \widetilde{P_{j}}-\sum e_{\sigma \tau}\left\langle\left(\partial^{\sigma} P^{\beta}\right)\left(\partial^{\tau} P_{j}\right) \mid P_{\gamma}^{*}\right\rangle \widetilde{P^{\gamma}}
$$

where the summation runs over all $\gamma \in B$ and all $\sigma, \tau \in \mathbb{N}^{n}$ such that $|\sigma| \geq$ $1,|\tau| \geq 1$. Remembering the homogeneity of all terms we see that the Fischer products in this sum vanish unless $\beta \cdot d-|\sigma|+d_{j}-|\tau|=\gamma \cdot d$, that is $\gamma \cdot d=\alpha \cdot d-(|\sigma|+|\tau|)<\alpha \cdot d$.

Assuming that $\widetilde{P^{\gamma}}=A_{\gamma}\left(e ; \widetilde{P}_{1}, \ldots, \widetilde{P}_{l}\right)$ for all $\gamma \in \mathbb{N}^{l}$ such that $\gamma \cdot d \leq N$ we deduce that $\widetilde{P^{\alpha}}=A_{\alpha}\left(e ; \widetilde{P_{1}}, \ldots, \widetilde{P_{l}}\right)$ for $\alpha \cdot d=N+1$, with

$$
A_{\alpha}=A_{\beta} \circ \widetilde{P_{j}}-\sum_{\gamma, \sigma, \tau} e_{\sigma \tau}\left\langle\left(\partial^{\sigma} P^{\beta}\right)\left(\partial^{\tau} P_{j}\right) \mid P_{\gamma}^{*}\right\rangle A_{\gamma},
$$

where the sum runs over all $\gamma \in B$ and $\sigma, \tau \in \mathbb{N}^{n}$ such that $|\sigma| \geq 1,|\tau| \geq 1$ and $\gamma \cdot d=\alpha \cdot d-(|\sigma|+|\tau|)$. All our claims now follow inductively.

\section{5 $e$-Functions and Integral Formulas}

Let $G / H$ be a symmetric space with $H$ compact (equipped with the normalized Haar measure $d h$ ). For the sake of simplicity we shall assume in this section that $\operatorname{Exp}: \mathfrak{s} \rightarrow G / H$ is a global diffeomorphism, so that the equality $\operatorname{Exp} Z(X, Y)=\exp X \cdot \operatorname{Exp} Y$ defines an analytic function $Z: \mathfrak{s} \times \mathfrak{s} \rightarrow \mathfrak{s}$; this is true for instance if $G / H$ is a Riemannian symmetric space of the noncompact type. We also assume that the $H$-invariant function $j$ is analytic and strictly positive on the whole $\mathfrak{s}$ (with $j(0)=1$ ); the function $j=J^{1 / 2}$ satisfies this condition.

We begin with an elementary lemma.

Lemma 3.15 (H compact) Let $f$ be a smooth function on $\mathfrak{s}$. The following are equivalent:

(i) $\langle u, f\rangle=0$ for any $H$-invariant distribution $u$ on $\mathfrak{s}$ such that $\operatorname{supp} u \cap \operatorname{supp} f$ is compact

(ii) $\int_{H} a(X) f(X) d X=0$ for any $H$-invariant test function a on $\mathfrak{s}$

(iii) $\int_{H} f(h \cdot X) d h=0$ for all $X \in \mathfrak{s}$.

Similarly, if $f$ is a smooth function on $\mathfrak{s} \times \mathfrak{s}$, the property $\langle u(X) v(Y), f(X, Y)\rangle$ $=0$ for every $H$-invariant distributions $u, v$ on $\mathfrak{s}(\operatorname{such}$ that $(\operatorname{supp} u \times \operatorname{supp} v) \cap$ supp $f$ is compact) is equivalent to $\int_{H \times H} f\left(h \cdot X, h^{\prime} \cdot Y\right) d h d h^{\prime}=0$ for all $X, Y \in \mathfrak{s}$.

Proof. (i) $\Rightarrow$ (ii). The distribution $u=a(X) d X$ fulfills the assumptions of (i). 
(ii) $\Rightarrow$ (iii). Let $a$ be any test function on $\mathfrak{s}$. Then $\int_{H} a\left(h^{-1} \cdot X\right) d h$ is $H$ invariant hence

$$
\int_{\mathfrak{s}} a(X) d X \int_{H} f(h \cdot X) d h=\int_{\mathfrak{s}} f(X) d X \int_{H} a\left(h^{-1} \cdot X\right) d h=0
$$

by (ii), which implies (iii).

(iii) $\Rightarrow$ (i) immediately, since

$$
\left\langle u(X), \int_{H} f(h \cdot X) d h\right\rangle=\int_{H}\langle u(X), f(h \cdot X)\rangle d h=\langle u, f\rangle
$$

by the $H$-invariance of $u$.

The next proposition, which makes essential use of $H$-invariant means, shows how an $e$-function can be obtained from a certain integral formula. In some cases (e.g. for rank one spaces, see Section 3.7 below) it leads to an explicit expression of $e$, which would be difficult to obtain from the general construction in Chapter 4.

Proposition 3.16 (H compact and Exp global diffeomorphism) Let $D: \mathfrak{s} \times$ $\mathfrak{s} \rightarrow \mathbb{R}$ be analytic, with $D(h \cdot X, h \cdot Y)=D(X, Y)$ for all $h \in H, X, Y \in \mathfrak{s}$. The following are equivalent:

(i) the function

$$
e(X, Y)=\frac{j(X) j(Y)}{j(X+Y)} D(X, Y)
$$

is an e-function of $G / H$

(ii) for any $H$-invariant smooth function $f$ on $\mathfrak{s}$ and any $X, Y \in \mathfrak{s}$,

$$
\int_{H} f(Z(X, h \cdot Y)) d h=\int_{H} f(X+h \cdot Y) D(X, h \cdot Y) d h
$$

(iii) for any $H$-bi-invariant smooth function $F$ on the group $G$ and any $X, Y \in \mathfrak{s}$,

$$
\int_{H} F\left(e^{X} e^{h \cdot Y}\right) d h=\int_{H} F\left(e^{X+h \cdot Y}\right) D(X, h \cdot Y) d h .
$$

Proof. The defining property (3.7) of $e$-functions, written with $e(X, Y)=$ $\frac{j(X) j(Y)}{j(X+Y)} D(X, Y)$,

$$
\langle u(X) \otimes v(Y), f(Z(X, Y))-D(X, Y) f(X+Y)\rangle=0
$$

is, by Lemma 3.15, equivalent to

$$
\int_{H \times H} f\left(Z\left(h \cdot X, h^{\prime} \cdot Y\right)\right) d h d h^{\prime}=\int_{H \times H} f\left(h \cdot X+h^{\prime} \cdot Y\right) D\left(h \cdot X, h^{\prime} \cdot Y\right) d h d h^{\prime}
$$


for any $f \in C^{\infty}(\mathfrak{s})$ and any $X, Y \in \mathfrak{s}$. Let $f_{H}(X)=\int_{H} f(h \cdot X) d h$. In view of the invariance of $D$ and $Z(h \cdot X, h \cdot Y)=h \cdot Z(X, Y)$, the change of variables $h^{\prime}=h h^{\prime \prime}$ in $\int_{H}(\ldots) d h^{\prime}$ transforms this property into

$$
\int_{H} f_{H}(Z(X, h \cdot Y)) d h=\int_{H} f_{H}(X+h \cdot Y) D(X, h \cdot Y) d h .
$$

Here $f_{H}$ is an arbitrary smooth $H$-invariant function on $\mathfrak{s}$, hence the equivalence of (i) and (ii).

Let $f$ be smooth and $H$-invariant. Defining $F$ by

$$
F(g)=f\left(\operatorname{Exp}^{-1}(g H)\right), g \in G,
$$

we obtain an $H$-bi-invariant smooth function on $G$. Conversely, any such $F$ may be obtained in this way from the $H$-invariant function $f=F \circ \exp$ on s. Since $\operatorname{Exp} Z(X, h \cdot Y)=e^{X} \cdot \operatorname{Exp}(h \cdot Y)$ this shows the equivalence of (ii) with (iii).

Remark. Applied to the mean value $f(X)=M^{X} \varphi(g H)$ (with $\varphi$ continuous), the result of (ii) implies the iterated mean value formula

$$
M^{X} M^{Y} \varphi(g H)=\int_{H} M^{X+h \cdot Y} \varphi(g H) D(X, h \cdot Y) d h .
$$

Corollary 3.17 (H compact and Exp global diffeomorphism) Under the assumptions stated at the beginning of this section, let $c: \mathfrak{s} \times \mathfrak{s} \rightarrow H$ be an analytic map such that

(i) $c(h \cdot X, h \cdot Y)=h c(X, Y) h^{-1}$ for any $h \in H, X, Y \in \mathfrak{s}$

(ii) for any $X \in \mathfrak{s}$, the Jacobian of the map $\psi_{X}: Y \mapsto c(X, Y) \cdot Y$ is strictly positive for $Y \in \mathfrak{s}$

(iii) for any $X, Y \in \mathfrak{s}, e^{X} e^{\psi_{X}(Y)}$ belongs to $H e^{X+Y} H$.

Then $\psi_{X}$ is a diffeomorphism of $\mathfrak{s}$ onto itself, and

$$
e(X, Y)=\frac{j(X) j(Y)}{j(X+Y)} \operatorname{det}_{\mathfrak{s}} D \psi_{X}(Y), X, Y \in \mathfrak{s}
$$

is an e-function for $G / H$, analytic on $\mathfrak{s} \times \mathfrak{s}$.

Remarks. (a) Such a map $c$ will be constructed in the next chapter (see Propositions $4.8(i), 4.10$ (iii) and 4.18 , with $\Omega=\mathfrak{s} \times \mathfrak{s}$ by Theorem $4.6(v)$ under our assumptions).

(b) Let $\|$.$\| be an H$-invariant norm on $\mathfrak{s}$ and $d$ the corresponding Riemannian distance on $S$, so that $d(o, \operatorname{Exp} X)=\|X\|$. Condition (iii), equivalent to $e^{X}$. $\operatorname{Exp} \psi_{X}(Y)=h \cdot \operatorname{Exp}(X+Y)$ for some $h \in H$, implies $d\left(o, e^{X} \cdot \operatorname{Exp} \psi_{X}(Y)\right)=$ $\|X+Y\|$, that is $d\left(\operatorname{Exp}(-X), \operatorname{Exp} \psi_{X}(Y)\right)=\|X+Y\|$.

Therefore $\operatorname{Exp} \psi_{X}(Y)=c(X, Y) \cdot \operatorname{Exp} Y$ must belong to the intersection of the sphere with center $o$ and radius $\|Y\|$ and the sphere with center $\operatorname{Exp}(-X)$ 
and radius $\|X+Y\|$. Details are given below for the 2-dimensional hyperbolic space $H^{2}(\mathbb{R})$.

Proof. By (i) $\psi_{h \cdot X}(h \cdot Y)=h \cdot \psi_{X}(Y)$, hence $D \psi_{h \cdot X}(h \cdot Y) \circ \operatorname{Ad} h=$ $\operatorname{Ad} h \circ D \psi_{X}(Y)$ and

$$
\operatorname{det} D \psi_{h \cdot X}(h \cdot Y)=\operatorname{det} D \psi_{X}(Y) .
$$

Thus $D(X, Y):=\operatorname{det} D \psi_{X}(Y)$ satisfies $D(h \cdot X, h \cdot Y)=D(X, Y)$.

Owing to the previous proposition we must check that

$$
\int_{H} F\left(e^{X} e^{h \cdot Y}\right) d h=\int_{H} F\left(e^{X+h \cdot Y}\right) \operatorname{det} D \psi_{X}(h \cdot Y) d h
$$

for any $H$-bi-invariant $F$. Taking an $H$-invariant norm on $\mathfrak{s}$ we have $\left\|\psi_{X}(Y)\right\|=$ $\|Y\|$; thus $\psi_{X}$ is a proper mapping and $\psi_{X}$ is a diffeomorphism of $\mathfrak{s}$ onto itself by (ii) and Hadamard's theorem.

Let $u$ be an $H$-invariant compactly supported continuous function on $\mathfrak{s}$. Then, using (ii) and (iii),

$$
\begin{aligned}
\int_{\mathfrak{s}} u(Y) d Y \int_{H} F\left(e^{X} e^{h \cdot Y}\right) d h & =\int_{\mathfrak{s}} F\left(e^{X} e^{Y}\right) u(Y) d Y \\
& =\int_{\mathfrak{s}} F\left(e^{X} e^{\psi_{X}(Y)}\right) u\left(\psi_{X}(Y)\right) \operatorname{det} D \psi_{X}(Y) d Y \\
& =\int_{\mathfrak{s}} u(Y) F\left(e^{X+Y}\right) \operatorname{det} D \psi_{X}(Y) d Y
\end{aligned}
$$

and the validity of this equality for any $H$-invariant $u$ implies (3.29) in view of Lemma 3.15 above.

Example. Consider the 2-dimensional hyperbolic space(the Poincaré disk $^{4}$ ) $H^{2}(\mathbb{R})=S U(1,1) / S O(2)$; the group $S U(1,1)$ acts on the unit disk of $\mathbb{C}$ by homographic transformations. Let $X=\left(\begin{array}{cc}0 & \xi \\ \bar{\xi} & 0\end{array}\right)$ and $Y=\left(\begin{array}{cc}0 & \eta \\ \bar{\eta} & 0\end{array}\right)$ denote two elements of $\mathfrak{s}$, with $\xi=\frac{x}{2} e^{i \alpha}, \eta=\frac{y}{2} e^{i \beta}, x, y \geq 0$ and $\alpha, \beta \in \mathbb{R}$. Then $\operatorname{Exp}(-X)=-\operatorname{th}(x / 2) e^{i \alpha}, \operatorname{Exp} Y=\operatorname{th}(y / 2) e^{i \beta}$ and, by Remark (b) above, we are looking for $c=c(X, Y)=\left(\begin{array}{cc}e^{i \theta} & 0 \\ 0 & e^{-i \theta}\end{array}\right) \in H, \theta \in \mathbb{R}$, such that $d(\operatorname{Exp}(-X), \operatorname{Exp}(c \cdot Y))=d(o, \operatorname{Exp}(X+Y))$.

Taking $\|X\|:=x$, which is an $H$-invariant norm on $\mathfrak{s}$, the corresponding metric on the unit disk $|w|<1$ of the complex plane is $d s^{2}=4\left(1-|w|^{2}\right)^{-2}|d w|^{2}$ and the hyperbolic distance $d\left(w, w^{\prime}\right)$ from $w$ to $w^{\prime}$ is given by

$$
\operatorname{th}\left(\frac{1}{2} d\left(w, w^{\prime}\right)\right)=\frac{\left|w^{\prime}-w\right|}{\left|1-\bar{w} w^{\prime}\right|} .
$$

\footnotetext{
${ }^{4}$ See [28], Introduction, for a detailed study of this example (with slightly different notation).
} 
Let $z:=\|X+Y\|=\left|x e^{i \alpha}+y e^{i \beta}\right|$. Condition (iii) becomes

$$
\left|\left(\operatorname{th} \frac{x}{2}\right) e^{i \alpha}+\left(\operatorname{th} \frac{y}{2}\right) e^{i(2 \theta+\beta)}\right|^{2}=\left|1+\left(\operatorname{th} \frac{x}{2} \operatorname{th} \frac{y}{2}\right) e^{i(2 \theta+\beta-\alpha)}\right|^{2}\left(\operatorname{th} \frac{z}{2}\right)^{2},
$$

that is

$$
\operatorname{sh} x \operatorname{sh} y \cos (2 \theta+\beta-\alpha)=\operatorname{ch} z-\operatorname{ch} x \operatorname{ch} y .
$$

The existence of a solution $c$, analytic function of $(X, Y)$, is guaranteed by Remark (a) above. The corresponding map $\psi_{X}$ is a rotation with angle $2 \theta$ (depending on $x, y, \alpha, \beta)$ in the complex plane. Its Jacobian $\operatorname{det} D \psi_{X}=$ $1+2 \partial_{\beta} \theta$ can be obtained by differentiation with respect to $\beta$ of (3.30) and

$$
z^{2}=x^{2}+y^{2}+2 x y \cos (\beta-\alpha) .
$$

It follows that

$$
\left(\operatorname{det} D \psi_{X}\right) \sin (2 \theta+\beta-\alpha)=\frac{x}{\operatorname{sh} x} \frac{y}{\operatorname{sh} y} \frac{\operatorname{sh} z}{z} \sin (\beta-\alpha),
$$

where the sines can be computed from (3.30) and (3.31); details are left to the Reader. Finally

$$
\left(\operatorname{det} D \psi_{X}\right)^{2}=\frac{1}{4}\left(\frac{\operatorname{sh} z}{z}\right)^{2} \frac{z^{2}-(x-y)^{2}}{\operatorname{ch} z-\operatorname{ch}(x-y)} \frac{(x+y)^{2}-z^{2}}{\operatorname{ch}(x+y)-\operatorname{ch} z} .
$$

The Jacobian of Exp is here $J(X)=\operatorname{sh} x / x$, therefore the $e$-function associated with $J^{1 / 2}$ given by Corollary 3.17 is, in the notation of Theorem 3.23 ,

$$
e(X, Y)=A(x, y, z)^{-1 / 2} .
$$

The norm used here is $\|X\|=x=\left(\frac{1}{2} B(X, X)\right)^{1 / 2}$ (as easily checked), thus our result agrees with Theorem 3.23 .

\section{6 e-Functions and Noncompact Symmetric Spaces}

In this section we specialize to the case of a Riemannian symmetric space $G / K$ of the noncompact type, where $G$ is a noncompact connected semisimple Lie group with finite center and $K$ is a maximal compact subgroup. Corollary 3.14 above relates spherical functions to $e$-functions by means of Taylor series expansions. Taking advantage of the semisimple structure we shall now discuss a different link which appears when going to infinity in the positive Weyl chamber, a method suggested to me by Mogens Flensted-Jensen. We propose two versions, the first with a limit of $e$ itself and the latter with a 
limit in $K$. Finally, we investigate possible extensions of the Duflo isomorphism to all convolution products. It should be noted that some results of the present section are partly conjectural and/or of a heuristic nature.

We shall need here several facts from the theory of semisimple Lie groups and refer to Helgason's books [27] and [28] for their proofs. Our general notation is put aside in this section, replaced by the classical semisimple notation in use in these references; please see Section 0.5. Besides, $\mathfrak{a}_{c}^{*}$ denotes the complexification of the dual $\mathfrak{a}^{*}, \pi: \mathfrak{p} \rightarrow \mathfrak{a}$ the orthogonal projection, $c(\lambda)$ Harish-Chandra's function and

$$
\varphi_{\lambda}(\operatorname{Exp} X)=\int_{K} e^{\left\langle i \lambda-\rho, A\left(k^{-1} \cdot X\right)\right\rangle} d k, \lambda \in \mathfrak{a}_{c}^{*},
$$

the spherical function, where the Iwasawa projection $A: \mathfrak{p} \rightarrow \mathfrak{a}$ corresponding to the Iwasawa decomposition $G=K A N$ is defined by $e^{X}=$ $k(X) e^{A(X)} n(X)$ with $X \in \mathfrak{p}, k(X) \in K, A(X) \in \mathfrak{a}$ and $n(X) \in N$.

\subsubsection{The Function $e_{\infty}$}

Theorem 3.18 ( $S=G / K$ of the noncompact type) Let $e$ be an e-function for $S$ associated to $j=J^{1 / 2}$, analytic in $\mathfrak{p} \times \mathfrak{p}$, and let $H \in \mathfrak{a}^{+}$be fixed. Assume the existence of

$$
e_{\infty}(X):=\lim _{t \rightarrow+\infty} e(X, t H)
$$

for all $X \in \mathfrak{p}$, with uniform convergence for $X$ running in any $K$-orbit in $\mathfrak{p}$. The spherical functions $\varphi_{\lambda}$ of $S$ are then given by

$$
J(X)^{1 / 2} \varphi_{\lambda}(\operatorname{Exp} X)=\int_{K} e^{i\left\langle\lambda, \pi\left(k^{-1} \cdot X\right)\right\rangle} e_{\infty}\left(k^{-1} \cdot X\right) d k
$$

for $\lambda \in \mathfrak{a}_{c}^{*}$ and $X \in \mathfrak{p}$.

Remarks. (a) The space $G / K$ actually has a globally defined $e$-function: see Theorem 3.23 for rank one spaces and Theorem 4.12 for general spaces, with $\Omega=\mathfrak{p} \times \mathfrak{p}$ by Theorem $4.6(v)$. The assumption about $e_{\infty}$ holds for rank one spaces (Proposition 3.24). and is obviously satisfied (with $e_{\infty}=1$ ) for a special symmetric space, that is if $G$ has a complex structure and $K$ is a compact real form (Proposition 3.7).

(b) Duistermaat's beautiful paper [20] gives a complete proof of a similar expression of the spherical functions of $G / K$. A few comments on his method are given at the end of 3.6.2.

(c) $e_{\infty}(m . X)=e_{\infty}(X)$ for $X \in \mathfrak{p}$ and $m \in M$, because $e(m \cdot X, H)=$ $e(m \cdot X, m \cdot H)=e(X, H)$ for $H \in \mathfrak{a}$. Owing to $\pi(m \cdot X)=\pi(X)$ the integral for $\varphi_{\lambda}$ is actually taken over $K / M$, with the $K$-invariant measure $d(k M)$ replacing $d k$. 
(d) In the rank one case (or for $G$ complex) we have $e(X, Y)=1$ whenever $[X, Y]=0$. Thus $e_{\infty}(X)=1$ for $X \in \mathfrak{a}$. The same is true for the $e$-function of Chapter 4 (Theorem 4.20) if the limit exists.

(e) Given $\lambda \in \mathfrak{a}_{c}^{*}$ let

$$
\psi_{\lambda}(X):=\int_{K} e^{i\left\langle\lambda, \pi\left(k^{-1} \cdot X\right)\right\rangle} e_{\infty}\left(k^{-1} \cdot X\right) d k .
$$

The theorem says that $\varphi_{\lambda}=\widetilde{\psi_{\lambda}}$ is a spherical function. According to the classical functional equation, this fact is equivalent to $\left\langle\widetilde{u} * \widetilde{v}, \widetilde{\psi_{\lambda}}\right\rangle=$ $\left\langle\widetilde{u}, \widetilde{\psi_{\lambda}}\right\rangle\left\langle\widetilde{v}, \widetilde{\psi_{\lambda}}\right\rangle$ for all $K$-invariant test functions $u, v$ on $\mathfrak{p}$, that is

$$
\left\langle u(X) v(Y), e(X, Y) \psi_{\lambda}(X+Y)\right\rangle=\left\langle u(X), \psi_{\lambda}(X)\right\rangle\left\langle v(Y), \psi_{\lambda}(Y)\right\rangle
$$

or else, by the definition of $\psi_{\lambda}$ and the $K$-invariance of $u, v$ and $e$,

$$
\left\langle u(X) v(Y),\left(e(X, Y) e_{\infty}(X+Y)-e_{\infty}(X) e_{\infty}(Y)\right) e^{i\langle\lambda, \pi(X+Y)\rangle}\right\rangle=0 .
$$

Conversely, a direct proof of this identity (for all $u, v, \lambda$ ) from the properties of the $e$-function would lead to a natural proof of the above theorem. It bears some similarity to an identity arising from the associativity $(\widetilde{u} * \widetilde{v}) * \widetilde{w}=$ $\widetilde{u} *(\widetilde{v} * \widetilde{w})$ for any $K$-invariant test functions $u, v, w$. Indeed, applied to a function $\widetilde{f}$ it implies by (3.4)

$$
\begin{aligned}
& <u(X) v(Y) w(Z) \\
& \quad,(e(X, Y) e(X+Y, Z)-e(X, Y+Z) e(Y, Z)) f(X+Y+Z)>=0
\end{aligned}
$$

or, by Lemma 3.15 on the variable $Z$,

$$
\langle u(X) v(Y),(e(X, Y) e(X+Y, Z)-e(X, Y+Z) e(Y, Z)) f(X+Y+Z)\rangle=0
$$

for any $Z \in \mathfrak{p}$ and $K$-invariant functions $u, v, f$; here again the $K$-invariance of $u, v$ and $e$ has been taken into account. It is now tempting to replace $Z$ by $t H, H \in \mathfrak{a}^{+}$, and let $t$ tend to $+\infty$ so as to prove (3.33)... For lack of a complete proof along these lines, we shall now infer Theorem 3.18 from Harish-Chandra's study of spherical functions.

(f) The spherical function is thus formally expressed as

$$
\varphi_{\lambda}(\operatorname{Exp} X)=\left.J(X)^{-1 / 2} e_{\infty}\left(\partial_{\xi}\right) \varphi_{\xi}^{0}(X)\right|_{\xi=i \lambda \circ \pi}
$$

where $\xi \in \mathfrak{p}_{c}^{*}$ (the complexified dual of $\mathfrak{p}$ ) and $\varphi_{\xi}^{0}(X)=\int_{K} e^{\left\langle\xi, k^{-1} \cdot X\right\rangle} d k$ is the generalized Bessel function.

Proof of Theorem 3.18. Proposition 3.16 and the functional equation of 
spherical functions imply

$$
\begin{aligned}
& J(X)^{1 / 2} \varphi_{\lambda}(\operatorname{Exp} X) \varphi_{\lambda}(\operatorname{Exp} t H)= \\
& \quad=\int_{K} \varphi_{\lambda}\left(\operatorname{Exp}\left(k^{-1} \cdot X+t H\right)\right)\left(\frac{J\left(k^{-1} \cdot X+t H\right)}{J(t H)}\right)^{1 / 2} e\left(k^{-1} \cdot X, t H\right) d k
\end{aligned}
$$

for $X \in \mathfrak{p}, H \in \mathfrak{a}, \lambda \in \mathfrak{a}_{c}^{*}$ and $t \in \mathbb{R}$. Referring to [28] Chapter IV for a detailed study of spherical functions, we shall estimate both sides as $t \rightarrow+\infty$ for $H \in \mathfrak{a}^{+}$, on the basis of Harish-Chandra's asymptotic formula:

$$
\lim _{t \rightarrow+\infty} e^{\langle\rho-i \lambda, t H\rangle} \varphi_{\lambda}(\operatorname{Exp} t H)=c(\lambda) .
$$

In order to replace this limit by a uniform estimate, we need some notation. The scalar product on $\mathfrak{p}$ induces one on $\mathfrak{a}$, hence an identification of $\mathfrak{a}$ with $\mathfrak{a}^{*}$ and a scalar product on $\mathfrak{a}^{*}$, still denoted by $\langle$,$\rangle . Let$

$$
\mathfrak{a}_{+}^{*}:=\left\{\lambda \in \mathfrak{a}^{*} \mid\langle\lambda, \alpha\rangle>0 \text { for all positive roots } \alpha\right\}
$$

be the positive Weyl chamber in $\mathfrak{a}^{*}$ and $\alpha_{1}, \ldots, \alpha_{l}$ denote the simple positive roots. Let $\mathfrak{a}_{c}^{\prime *}$ be a specific $W$-invariant connected dense open subset of $\mathfrak{a}_{c}^{*}$, the complement of a union of countably many hyperplanes, on which $c(\lambda)$ is holomorphic ([28] p. 434). We first prove two lemmas.

Lemma 3.19 Given $\varepsilon>0$ and $\lambda \in \mathfrak{a}_{c}^{\prime *}$ such that $-\operatorname{Im} \lambda \in \mathfrak{a}_{+}^{*}$, there exists $C>0$ such that $H \in \mathfrak{a}$ and $\alpha_{1}(H) \geq C, \ldots, \alpha_{l}(H) \geq C$ imply

$$
\left|e^{\langle\rho-i \lambda, H\rangle} \varphi_{\lambda}(\operatorname{Exp} H)-c(\lambda)\right| \leq \varepsilon .
$$

Proof. Harish-Chandra's expansion for $\varphi_{\lambda}$ gives ([28] p. 430)

$$
e^{\langle\rho, H\rangle} \varphi_{\lambda}(\operatorname{Exp} H)=\sum_{w \in W} c(w \lambda) e^{\langle i w \lambda, H\rangle} \sum_{\mu \in \Lambda} \Gamma_{\mu}(w \lambda) e^{-\langle\mu, H\rangle}
$$

for $H \in \mathfrak{a}^{+}, \lambda \in \mathfrak{a}_{c}^{\prime *}$, where $\Lambda$ is the set of all $n_{1} \alpha_{1}+\cdots+n_{l} \alpha_{l}$ with $n_{1}, \ldots, n_{l} \in$ $\mathbb{N}$ and the coefficients $\Gamma_{\mu}$ are obtained inductively from $\Gamma_{0}=1$. The recursion formula implies that for any $\lambda$ as above and any $H_{0} \in \mathfrak{a}^{+}$there exists a constant $M>0$ (depending on $\lambda$ and $H_{0}$ ) such that $\left|c(w \lambda) \Gamma_{\mu}(w \lambda)\right| \leq M e^{\left\langle\mu, H_{0}\right\rangle}$ for all $\mu \in \Lambda, w \in W$ ([28] p. 428). Separating the term with $w=e$ and $\mu=0$ we obtain

$$
\begin{aligned}
& e^{\langle\rho-i \lambda, H\rangle} \varphi_{\lambda}(\operatorname{Exp} H)-c(\lambda)= \\
& =c(\lambda) \sum_{\mu \neq 0} \Gamma_{\mu}(\lambda) e^{-\langle\mu, H\rangle}+\sum_{w \neq e} c(w \lambda) e^{i\langle w \lambda-\lambda, H\rangle} \sum_{\mu} \Gamma_{\mu}(w \lambda) e^{-\langle\mu, H\rangle},
\end{aligned}
$$


whence the estimate

$$
\begin{aligned}
& \left|e^{\langle\rho-i \lambda, H\rangle} \varphi_{\lambda}(\operatorname{Exp} H)-c(\lambda)\right| \leq \\
& \leq M\left(\sum_{\mu \neq 0} e^{\left\langle\mu, H_{0}-H\right\rangle}+\sum_{w \neq e} e^{\langle\operatorname{Im} \lambda-w \operatorname{Im} \lambda, H\rangle} \sum_{\mu} e^{\left\langle\mu, H_{0}-H\right\rangle}\right) \\
& \quad \leq M\left(f\left(H-H_{0}\right)-1+f\left(H-H_{0}\right) \sum_{w \neq e} e^{\langle\operatorname{Im} \lambda-w \operatorname{Im} \lambda, H\rangle}\right)
\end{aligned}
$$

with $f(H):=\sum_{\mu \in \Lambda} e^{-\langle\mu, H\rangle}=\prod_{j=1}^{l}\left(1-e^{-\left\langle\alpha_{j}, H\right\rangle}\right)^{-1}$.

Fixing $H_{0}$ we have $0<f\left(H-H_{0}\right)-1 \leq \eta$ for any given $\eta>0$ if $\alpha_{1}(H), \ldots, \alpha_{l}(H)$ are large enough. Furthermore the assumption $-\operatorname{Im} \lambda \in \mathfrak{a}_{+}^{*}$ implies that, in the basis of $\mathfrak{a}^{*}$ given by the simple roots, $w \operatorname{Im} \lambda-\operatorname{Im} \lambda=\sum_{j=1}^{l} \lambda_{j} \alpha_{j}$ with coefficients $\lambda_{j} \geq 0$, not all 0 if $w \neq e$ (see [27] p. 292-293). The lemma follows.

Lemma 3.20 Let $C$ be a compact subset of $\mathfrak{p}$. Given $X \in C$ and $H \in \mathfrak{a}^{+}$, (i) there exists $k(t) \in K$ such that

$$
X+t H=k(t) \cdot(t H+\pi(X)+\varepsilon(t))
$$

with $\varepsilon(t) \in \mathfrak{a}$ and $\varepsilon(t)=O\left(t^{-1}\right)$ as $t \rightarrow+\infty$, uniformly for $X \in C$

(ii) $(J(X+t H) / J(t H))^{1 / 2} \rightarrow e^{\langle\rho, \pi(X)\rangle}$ as $t \rightarrow+\infty$, uniformly for $X \in C$.

Proof. (i) Let $u=t^{-1}$. Since $H \in \mathfrak{a}^{+}$, for $t$ large enough $H+u X$ belongs to the open set $\mathfrak{p}^{\prime}$ of regular elements. By the polar decomposition on $\mathfrak{p}^{\prime}$, a diffeomorphism of $K / M \times \mathfrak{a}^{+}$onto $\mathfrak{p}^{\prime}$ ([28] p. 195), there exist two functions ${ }^{5}$ $Z(u) \in \mathfrak{k}$ and $R(u) \in \mathfrak{a}^{+}$, smooth near $u=0$ and such that

$$
H+u X=e^{Z(u)} \cdot R(u), Z(0)=0, R(0)=H .
$$

The derivative at $u=0$ is $X=R^{\prime}(0)+\left[Z^{\prime}(0), H\right]$. The latter term, which belongs to $[\mathfrak{k}, \mathfrak{a}]$, is orthogonal to $\mathfrak{a}$ in view of the invariance of the Killing form together with $[\mathfrak{a}, \mathfrak{a}]=0$. Therefore $R^{\prime}(0)=\pi(X)$ and

$$
H+u X=e^{Z(u)} \cdot\left(H+u \pi(X)+O\left(u^{2}\right)\right) .
$$

The result follows by multiplication by $t$. The remainder depends smoothly on $X$ and the estimate is uniform for $X \in C$.

(ii) By (i) $J(X+t H)=J(H(t))$ with $H(t)=t H+\pi(X)+\varepsilon(t) \in \mathfrak{a}$, therefore

$$
\left(\frac{J(X+t H)}{J(t H)}\right)^{1 / 2}=\prod_{\alpha>0}\left(\frac{\operatorname{sh}\langle\alpha, H(t)\rangle}{\operatorname{sh} t\langle\alpha, H\rangle} \frac{t\langle\alpha, H\rangle}{\langle\alpha, H(t)\rangle}\right)^{m_{\alpha} / 2},
$$

${ }^{5}$ We choose a section of $K \rightarrow K / M$ in a neighborhhod of the origin. 
a product taken over all positive roots $\alpha$.

Since $\langle\alpha, H\rangle>0$ we have $\langle\alpha, H(t)\rangle / t\langle\alpha, H\rangle \rightarrow 1$ and

$$
\frac{\operatorname{sh}\langle\alpha, H(t)\rangle}{\operatorname{sh} t\langle\alpha, H\rangle}=e^{\langle\alpha, \pi(X)+\varepsilon(t)\rangle} \frac{1-e^{-2\langle\alpha, H(t)\rangle}}{1-e^{-2 t\langle\alpha, H\rangle}} \rightarrow e^{\langle\alpha, \pi(X)\rangle}
$$

for $t \rightarrow+\infty$, uniformly for $X \in C$. The result follows, with $\rho=\frac{1}{2} \sum m_{\alpha} \alpha$.

Going back to the proof of Theorem 3.18 we rewrite (3.35) as

$$
\begin{aligned}
& J(X)^{1 / 2} \varphi_{\lambda}(\operatorname{Exp} X) e^{\langle\rho-i \lambda, t H\rangle} \varphi_{\lambda}(\operatorname{Exp} t H)= \\
& \int_{K} e^{\langle\rho-i \lambda, t H\rangle} \varphi_{\lambda}\left(\operatorname{Exp}\left(k^{-1} \cdot X+t H\right)\right)\left(\frac{J\left(k^{-1} \cdot X+t H\right)}{J(t H)}\right)^{1 / 2} e\left(k^{-1} \cdot X, t H\right) d k
\end{aligned}
$$

and apply Lemma 3.19 to both sides of this equation. Given $H \in \mathfrak{a}^{+}, \lambda \in \mathfrak{a}_{c}^{\prime *}$ and $-\operatorname{Im} \lambda \in \mathfrak{a}_{+}^{*}$ the left-hand side tends to $c(\lambda) J(X)^{1 / 2} \varphi_{\lambda}(\operatorname{Exp} X)$ as $t \rightarrow$ $+\infty$. By Lemma $3.20(i)$ in the right-hand side $\varphi_{\lambda}\left(\operatorname{Exp}\left(k^{-1} \cdot X+t H\right)\right)=$ $\varphi_{\lambda}(\operatorname{Exp} H(t))$ with $H(t)=t H+\pi\left(k^{-1} \cdot X\right)+\varepsilon(t)$ and $\varepsilon(t) \rightarrow 0$ in $\mathfrak{a}$ as $t \rightarrow+\infty$, uniformly for $k \in K$. Then $e^{\langle\rho-i \lambda, H(t)\rangle} \varphi_{\lambda}(\operatorname{Exp} H(t)) \longrightarrow c(\lambda)$ hence

$$
e^{\langle\rho-i \lambda, t H\rangle} \varphi_{\lambda}\left(\operatorname{Exp}\left(k^{-1} \cdot X+t H\right)\right) \longrightarrow e^{\left\langle i \lambda-\rho, \pi\left(k^{-1} \cdot X\right)\right\rangle} c(\lambda)
$$

since $e^{\varepsilon(t)} \rightarrow 1$ (uniformly in $k$ ). Besides

$$
\left(\frac{J\left(k^{-1} \cdot X+t H\right)}{J(t H)}\right)^{1 / 2} e\left(k^{-1} \cdot X, t H\right) \longrightarrow e^{\left\langle\rho, \pi\left(k^{-1} \cdot X\right)\right\rangle} e_{\infty}\left(k^{-1} \cdot X\right)
$$

by Lemma 3.20 (ii), uniformly for $k \in K$. Limits can thus be taken under the integral sign, which implies our claim for $\lambda \in \mathfrak{a}_{c}^{\prime *}$ and $-\operatorname{Im} \lambda \in \mathfrak{a}_{+}^{*}$ (the factors $c(\lambda)$ cancel out), hence for all $\lambda \in \mathfrak{a}_{c}^{*}$ by analytic continuation.

\subsubsection{Link with the Iwasawa Projection}

Let us take a closer look at the function $e_{\infty}$. Still working on a Riemannian symmetric space $G / K$ of the noncompact type with $j=J^{1 / 2}$, let $c: \mathfrak{p} \times \mathfrak{p} \rightarrow K$ and $\psi_{X}(Y):=c(X, Y) \cdot Y$ satisfy the assumptions of Corollary 3.17. Together with Lemma 3.20 (ii) it shows that, for $H \in \mathfrak{a}^{+}, e_{\infty}(X)=\lim _{t \rightarrow+\infty} e(X, t H)$ exists if and only if $D(X):=\lim _{t \rightarrow+\infty} \operatorname{det}_{\mathfrak{p}} D \psi_{X}(t H)$ exists; then

$$
e_{\infty}(X)=J(X)^{1 / 2} e^{-\langle\rho, \pi(X)\rangle} D(X) .
$$

By Theorem 3.18 the spherical functions are then

$$
\varphi_{\lambda}(\operatorname{Exp} X)=\int_{K} e^{\left\langle i \lambda-\rho, \pi\left(k^{-1} \cdot X\right)\right\rangle} D\left(k^{-1} \cdot X\right) d k
$$


where $X \in \mathfrak{p}$ and $\lambda$ runs over the complexified dual $\mathfrak{a}_{c}^{*}$. As noted in 3.6.1 Remark $(c)$, this is actually an integral over $K / M$.

We now try to compare this expression with Harish-Chandra's classical formula

$$
\varphi_{\lambda}(\operatorname{Exp} X)=\int_{K} e^{\left\langle i \lambda-\rho, A\left(k^{-1} \cdot X\right)\right\rangle} d k,
$$

where $A$ is the Iwasawa projection defined at the beginning of this section. Since $M$ normalizes $N$ we have $A(m \cdot X)=A(X)$ and this is again an integral over $K / M$.

The link between both formulas for $\varphi_{\lambda}$ can be understood by means of a new expression of $\operatorname{det} D \psi_{X}$. The «polar coordinates» map $(k M, H) \mapsto$ $Y=k \cdot H$ is a diffeomorphism of $K / M \times \mathfrak{a}^{+}$onto the dense open subset $\mathfrak{p}^{\prime}$ of regular elements in $\mathfrak{p}$, with Jacobian $\Pi \alpha(H)^{m_{\alpha}}$ (product taken over all positive roots $\alpha$; see [28] p. 195). Restricted to $\mathfrak{p}^{\prime}$ the map $\psi_{X}$ decomposes as

$$
Y \longmapsto(k M, H) \longmapsto(c(X, k \cdot H) k M, H) \longmapsto c(X, k \cdot H) k \cdot H=\psi_{X}(Y) .
$$

The Jacobians of the first, resp. third, map are $\Pi \alpha(H)^{-m_{\alpha}}$, resp. $\Pi \alpha(H)^{m_{\alpha}}$, therefore $\operatorname{det} D \psi_{X}(Y)$ is the Jacobian of the map $k M \mapsto \gamma(k) M$ of $K / M$ into itself with $H \in \mathfrak{a}^{+}$fixed, $X \in \mathfrak{p}$ and $\gamma(k):=c(X, k \cdot H) k=k c\left(k^{-1} \cdot X, H\right)$. By Corollary 3.17 this map is, for each $H \in \mathfrak{a}^{+}$, a diffeomorphism of $K / M$ onto itself. Its Jacobian actually means the Jacobian of the map taken back to the origin $o$ of $K / M$ by the action of $K$ (see [28] p. 93), that is $k^{\prime} M \mapsto$ $\gamma(k)^{-1} \gamma\left(k k^{\prime}\right) M$ at $k^{\prime} M=o$. Since $\gamma(k m)=\gamma(k) m$ for $k \in K$ and $m \in M$, the restriction to $M$ of the map $k^{\prime} \mapsto \gamma(k)^{-1} \gamma\left(k k^{\prime}\right)$ is the identity. The tangent space to $K / M$ at $o$ identifies with the orthogonal of $\mathfrak{m}$ in $\mathfrak{k}$, and it follows that our Jacobian equals the Jacobian of the latter map at $k^{\prime}=e$ in $K$. Remembering the definition of $\gamma$ we finally obtain $\left(D_{k^{\prime}}\right.$ meaning the differential with respect to $k^{\prime}$ )

$$
\operatorname{det}_{\mathfrak{p}} D \psi_{X}(Y)=\left.\operatorname{det}_{\mathfrak{k}} D_{k^{\prime}}\left(c\left(k^{-1} \cdot X, H\right)^{-1} k^{\prime} c\left(k^{\prime-1} k^{-1} \cdot X, H\right)\right)\right|_{k^{\prime}=e}
$$

with $X \in \mathfrak{p}, Y=k \cdot H, k \in K, H \in \mathfrak{a}^{+}$; it only depends on $k^{-1} \cdot X$ and $H$. In particular, for $k=e$,

$$
\operatorname{det}_{\mathfrak{p}} D \psi_{X}(H)=\left.\operatorname{det}_{\mathfrak{k}} D_{k^{\prime}}\left(c(X, H)^{-1} k^{\prime} c\left(k^{\prime-1} \cdot X, H\right)\right)\right|_{k^{\prime}=e} .
$$

Replacing now $H$ by $t H$ with $t>0$ we discuss the behavior as $t \rightarrow+\infty$. For any $X \in \mathfrak{p}$ and any sequence $t_{n} \rightarrow+\infty$ the sequence $c\left(X, t_{n} H\right)$ in the compact group $K$ admits a convergent subsequence; its limit is called a limit point of $c(X, t H)$ as $t \rightarrow+\infty$.

Proposition 3.21 Let $c: \mathfrak{p} \times \mathfrak{p} \rightarrow K$ be such that for any $X, Y \in \mathfrak{p}$, $e^{X} e^{c(X, Y) \cdot Y}$ belongs to $K e^{X+Y} K$ (assumption (iii) of Corollary 3.17).

Fixing $H \in \mathfrak{a}^{+}$let $c(X)$, for $X \in \mathfrak{p}$, denote a limit point of $c(X, t H)$ in $K$ as $t$ tends to $+\infty$. The Iwasawa projection $A$ and the orthogonal projection $\pi$ are then linked by

$$
A\left(c(X)^{-1} \cdot X\right)=\pi(X)
$$


The proof rests on the following lemma, linking the orthogonal projection $\pi: \mathfrak{p} \rightarrow \mathfrak{a}$ and the Iwasawa projection $A: \mathfrak{p} \rightarrow \mathfrak{a}$ with the radial component $R: G \rightarrow \overline{\mathfrak{a}^{+}}$defined by the Cartan decomposition ([27] p. 402)

$$
g=k e^{R(g)} k^{\prime}
$$

for some $k, k^{\prime} \in K$.

Lemma 3.22 For $X \in \mathfrak{p}, H \in \mathfrak{a}^{+}$we have the following asymptotic expansions of radial components as $t$ tends to $+\infty$ :

$$
\begin{aligned}
& R\left(e^{X+t H}\right)=t H+\pi(X)+O\left(t^{-1}\right) \\
& R\left(e^{X} e^{t H}\right)=t H+A(X)+O\left(e^{-t \mu(H)}\right)
\end{aligned}
$$

with $\mu(H):=\inf _{\alpha>0} \alpha(H)>0$ (infimum over the set of positive roots $\alpha$ ). These estimates are uniform for $X$ in a compact subset of $\mathfrak{p}$.

Proof. The first result is a restatement of Lemma $3.20(i)$. The latter is proved in Appendix A.

Proof of Proposition 3.21. The assumption on $c$ implies the equality of radial components

$$
R\left(e^{c(X, t H)^{-1} \cdot X} e^{t H}\right)=R\left(e^{X+t H}\right)
$$

and Lemma 3.22 gives

$$
t H+A\left(c(X, t H)^{-1} \cdot X\right)+O\left(e^{-t \mu(H)}\right)=t H+\pi(X)+O\left(t^{-1}\right),
$$

whence $A\left(c(X, t H)^{-1} \cdot X\right)=\pi(X)+O\left(t^{-1}\right)$ and the conclusion.

Formulas (3.36) and (3.37) for spherical functions can now be compared by the following heuristic arguments. The factor $D(X)$ in (3.36) is $D(X)=$ $\lim _{t \rightarrow+\infty} \operatorname{det}_{\mathfrak{p}} D \psi_{X}(t H)$. From (3.38) we may expect that, in the notation of Proposition 3.21,

$$
D(X)=\left.\operatorname{det}_{\mathfrak{k}} D_{k^{\prime}}\left(c(X)^{-1} k^{\prime} c\left(k^{\prime-1} \cdot X\right)\right)\right|_{k^{\prime}=e}
$$

(heuristic formula). For $X \in \mathfrak{p}$ and $k \in K$ let

$$
\Phi(X, k):=k c\left(k^{-1} \cdot X\right)=k \Phi\left(k^{-1} \cdot X, e\right),
$$

a limit point in $K$ of $k c\left(k^{-1} \cdot X, t H\right)=c(X, t k \cdot H) k$ as $t$ tends to $+\infty$ with $H \in \mathfrak{a}^{+}$. As above for $\gamma$ the Jacobian $\operatorname{det} D_{k} \Phi(X, k)$ of the map $k \mapsto \Phi(X, k)$ (assumed to be smooth) is actually

$$
\begin{aligned}
\left.\operatorname{det}_{\mathfrak{k}} D_{k^{\prime}}\left(\Phi(X, k)^{-1} \Phi\left(X, k k^{\prime}\right)\right)\right|_{k^{\prime}=e}= & \\
=\left.\operatorname{det}_{\mathfrak{k}} D_{k^{\prime}}\left(c\left(k^{-1} \cdot X\right)^{-1} k^{\prime} c\left(k^{\prime-1} k^{-1} \cdot X\right)\right)\right|_{k^{\prime}=e} & =D\left(k^{-1} \cdot X\right) .
\end{aligned}
$$


Formula (3.36) becomes

$$
\varphi_{\lambda}(\operatorname{Exp} X)=\int_{K} e^{<i \lambda-\rho, \pi\left(k^{-1} \cdot X\right)>} \operatorname{det} D_{k} \Phi(X, k) d k
$$

with $\operatorname{det} D_{k} \Phi(X, k)=D\left(k^{-1} \cdot X\right)$. But $\pi\left(k^{-1} \cdot X\right)=A\left(\Phi(X, k)^{-1} \cdot X\right)$ by Proposition 3.21 and the change of variable $k \mapsto \Phi(X, k)$ finally gives a (heuristic) proof of Harish-Chandra's formula (3.37).

A rigorous proof of (3.39) from (3.37) was given by Duistermaat [20] by means of a direct construction of a map $k \mapsto \Psi(X, k)$, similar to $\Phi$, such that $\pi\left(k^{-1} \cdot X\right)=A\left(\Psi(X, k)^{-1} \cdot X\right)$. He obtained it as the value for $t=1$ of a oneparameter family of diffeomorphisms $k \mapsto \Psi_{t}(X, k)$, the flow of a carefully chosen time-dependent vector field $v_{t}(X, k)$ on $K$ (or $\left.K / M\right)$; his construction of $v_{t}$ uses the root system of $(\mathfrak{g}, \mathfrak{a})$. Besides $\Psi(X, k)=k \Psi\left(k^{-1} \cdot X, e\right)$ and, as above for $\Phi$, the Jacobian $\operatorname{det} D_{k} \Psi(X, k)$ only depends on $k^{-1} \cdot X$.

On the other hand Lemma 4.7 below proposes a construction of our $c(X, Y)$ by means of a vector field $C_{t}(X, Y)$ on $K$ depending on the parameters $X, Y$; Duistermaat's vector field $v_{t}$ might thus be a limit of ours as $Y$ goes to infinity in $\mathfrak{a}^{+}$. Turning this into a satisfactory argument seems difficult however, for lack of an explicit definition of our $C_{t}(X, Y)$.

\subsubsection{Extension of Duflo's Isomorphism}

Remembering the quote from Helgason in the preface, one may expect that an $e$-function for $S=G / H$ provides the appropriate tool to construct an isomorphism $\mathcal{T}$ of a space of $H$-invariant distributions on $S$ onto a space of $H$ invariant distributions on its tangent space $\mathfrak{s}$, so that $\mathcal{T}\left(U *_{S} V\right)=\mathcal{T} U *_{\mathfrak{s}} \mathcal{T} V$ for all $H$-invariant $U, V$. This isomorphism should encompass both the Abel transform of symmetric spaces of the noncompact type and the map $\sim$ of special spaces. Specializing to the case $\operatorname{supp} V=\{0\}$, this would imply the existence of a fundamental solution for all operators in $\mathbb{D}(S)$, hence their local solvability, and $\operatorname{supp} U=\operatorname{supp} V=\{0\}$ would give a generalized Duflo isomorphism (cf. Section 3.3.4). An obvious necessary condition for that is the commutativity of the convolution $*_{S}$ (and of $\mathbb{D}(S)$ in particular), which holds true if $e$ is symmetric: $e(X, Y)=e(Y, X)$.

We discuss here this issue for a Riemannian symmetric space $S=G / K$ of the noncompact type, keeping to the semisimple notation of 0.5 and the previous subsections.

a. Using spherical harmonic analysis on $S$. This will easily lead to a first construction of $\mathcal{T}$. We recall some classical results from [28] Chapter IV. Let $f$ be a $K$-invariant test function on $S$, with supp $f$ contained in the ball with center $o$ and radius $R$. Its spherical transform $\mathcal{F}_{S} f$, defined by

$$
\mathcal{F}_{S} f(\lambda):=\int_{S} f(x) \varphi_{-\lambda}(x) d x
$$


where $\lambda \in \mathfrak{a}_{c}^{*}$ and $\varphi_{-\lambda}$ is the spherical function (3.37), is a Weyl group invariant entire function of exponential type $R$. A linear form on $\mathfrak{a}$ identifies with a linear form on $\mathfrak{p}$ vanishing on the orthogonal of $\mathfrak{a}$ in $\mathfrak{p}$, thus $\mathfrak{a}_{c}^{*}$ may be identified with a subspace of $\mathfrak{p}_{c}^{*}$. By the «holomorphic Chevalley theorem» in [28] p. $468, \mathcal{F}_{S} f$ extends uniquely to a $K$-invariant entire function on $\mathfrak{p}_{c}^{*}$ of the same exponential type, therefore the Fourier transform of a test function $\mathcal{T} f$ on $\mathfrak{p}$ with $\operatorname{supp} \mathcal{T} f$ contained in the ball with center 0 and radius $R$ :

$$
\mathcal{F}_{S} f(\xi)=\mathcal{F}_{\mathfrak{p}} \mathcal{T} f(\xi) \text { with } \mathcal{F}_{\mathfrak{p}} u(\xi):=\int_{\mathfrak{p}} u(X) e^{-i\langle\xi, X\rangle} d X, \xi \in \mathfrak{p}_{c}^{*} .
$$

By the functional equation for $\varphi_{-\lambda}$ we have $\mathcal{F}_{S}\left(f *_{S} g\right)(\lambda)=\mathcal{F}_{S} f(\lambda) . \mathcal{F}_{S} g(\lambda)$ for $\lambda \in \mathfrak{a}_{c}^{*}$, which extends to $\xi \in \mathfrak{p}_{c}^{*}$ and gives

$$
\mathcal{T}\left(f *_{S} g\right)=\mathcal{T} f *_{\mathfrak{p}} \mathcal{T} g
$$

for all $K$-invariant test functions $f, g$ on $S$. Because $\varphi_{-\lambda}$ is an eigenfunction of all differential operators $D \in \mathbb{D}(S)$, we have $\mathcal{F}_{S}(D f)(\lambda)=\Gamma(D)(i \lambda) \mathcal{F}_{S} f(\lambda)$ where $\Gamma: \mathbb{D}(S) \rightarrow S(\mathfrak{a})^{W}$ is Harish-Chandra's isomorphism. By Chevalley's theorem again, $\lambda \mapsto \Gamma(D)(i \lambda)$ extends to a $K$-invariant polynomial on $\mathfrak{p}^{*}$ and we infer that there exists a differential operator $\mathcal{T}(D) \in \mathbb{D}(\mathfrak{p})^{K}$ such that

$$
\mathcal{T}(D f)=\mathcal{T}(D) \mathcal{T} f
$$

The operator $\mathcal{T}$ can also be obtained from the Abel transform $\mathcal{A}$ as follows. Remembering the Iwasawa decomposition $G=K A N$ we set

$$
\mathcal{A} f(H):=e^{\langle\rho, H\rangle} \int_{N} f\left(e^{H} n K\right) d n, H \in \mathfrak{a},
$$

for $K$-invariant $f$. It easily follows from (3.37) that, for $\lambda \in \mathfrak{a}_{c}^{*}$,

$$
\mathcal{F}_{\mathfrak{p}} \mathcal{T} f(\lambda)=\mathcal{F}_{S} f(\lambda)=\mathcal{F}_{\mathfrak{a}} \mathcal{A} f(\lambda), \text { with } \mathcal{F}_{\mathfrak{a}} u(\lambda):=\int_{\mathfrak{a}} u(H) e^{-i\langle\lambda, H\rangle} d H .
$$

Let $\mathfrak{q}$ denote the orthogonal complement of $\mathfrak{a}$ in $\mathfrak{p}$ and $X=H+Y$ with $H \in \mathfrak{a}, Y \in \mathfrak{q}$, the corresponding decomposition of $X \in \mathfrak{p}$. Then

$$
\mathcal{F}_{\mathfrak{a}} \mathcal{A} f(\lambda)=\int_{\mathfrak{a} \times \mathfrak{q}} \mathcal{T} f(H+Y) e^{-i\langle\lambda, H\rangle} d H d Y=\mathcal{F}_{\mathfrak{a}} \mathcal{A}_{0} \mathcal{T} f(\lambda)
$$

where $\mathcal{A}_{0}$ is the «flat Abel transform» defined (for $K$-invariant functions) by

$$
\mathcal{A}_{0} u(H):=\int_{\mathfrak{q}} u(H+Y) d Y,
$$

whence $\mathcal{A}=\mathcal{A}_{0} \mathcal{T}$ by the injectivity of $\mathcal{F}_{\mathfrak{a}}$ and finally $\mathcal{T}=\mathcal{A}_{0}^{-1} \mathcal{A}$ since $\mathcal{A}_{0}$ is invertible by Helgason's Theorem 5.3 of [29], Chapter IV. An explicit formula for $\mathcal{T}$ can be extracted from the proof of this theorem. Let $\omega(\lambda):=$ 
$\prod_{\alpha>0}\langle\alpha, \lambda\rangle^{m_{\alpha}}$ (product over all positive roots) and let $|\omega(\partial)|$ denote the pseudo-differential operator on $\mathfrak{a}$ with symbol $|\omega(\lambda)|$, that is $\mathcal{F}_{\mathfrak{a}}(|\omega(\partial)| u)(\lambda)=$ $|\omega(\lambda)| \mathcal{F}_{\mathfrak{a}} u(\lambda)$. If all multiplicities $m_{\alpha}$ are even, $|\omega(\partial)|$ is a differential operator; otherwise it is a differential operator composed with a Hilbert transform. Helgason's formula implies, for $f \in \mathcal{D}(S)^{K}$,

$$
\mathcal{T} f(X)=C \int_{K / M}(|\omega(\partial)| \mathcal{A} f)\left(\pi\left(k^{-1} \cdot X\right)\right) d(k M),
$$

where $C$ is a constant and $\pi: \mathfrak{p} \rightarrow \mathfrak{a}$ is the orthogonal projection.

Summing up, we see that the linear bijection

$$
\mathcal{T}=\mathcal{F}_{\mathfrak{p}}^{-1} \circ \mathcal{F}_{S}=\mathcal{A}_{0}^{-1} \circ \mathcal{A}: \mathcal{D}(S)^{K} \rightarrow \mathcal{D}(\mathfrak{p})^{K},
$$

defined by means of spherical harmonic analysis on $S=G / K$, satisfies

$$
\mathcal{T}\left(f *_{S} g\right)=\mathcal{T} f *_{\mathfrak{p}} \mathcal{T} g, \mathcal{T}(D f)=\mathcal{T}(D) \mathcal{T} f .
$$

b. Using an $e$-function. We now try to link $\mathcal{T}$ with an $e$-function of $S$ associated to $j=J^{1 / 2}$, assuming here the existence of $e_{\infty}$ (see Theorem 3.18 ), smooth on a neighborhhod of the origin in $\mathfrak{p}$. The spherical functions are then $\varphi_{\lambda}=\widetilde{\psi}_{\lambda}$ with

$$
\psi_{\lambda}(X):=\int_{K} e^{i\left\langle\lambda, \pi\left(k^{-1} \cdot X\right)\right\rangle} e_{\infty}\left(k^{-1} \cdot X\right) d k, \lambda \in \mathfrak{a}_{c}^{*}, X \in \mathfrak{p} .
$$

As already mentioned, a similar formula has been proved by Duistermaat [20], with $e_{\infty}$ replaced by some (smooth) Jacobian.

Since $\varphi_{\lambda}$ is an eigenfunction of all operators in $\mathbb{D}(S)$ we have $\widetilde{P} \widetilde{\psi}_{\lambda}=c(P, \lambda) \widetilde{\psi}_{\lambda}$ for $P \in \mathbb{D}(\mathfrak{p})^{K}$, where the scalar $c(P, \lambda)$ is given by

$$
\begin{aligned}
c(P, \lambda) & =\widetilde{P} \widetilde{\psi}_{\lambda}(o)=P \psi_{\lambda}(0)=\left.P\left(\partial_{X}\right)\left(e^{i\langle\lambda, \pi(X)\rangle} e_{\infty}(X)\right)\right|_{X=0} \\
& =\left(P\left(\partial_{X}+i \lambda\right) e_{\infty}\right)(0)
\end{aligned}
$$

in view of the $K$-invariance of $P$ (here $\lambda \in \mathfrak{a}^{*}$ is identified with $\lambda \circ \pi$, a linear form on $\mathfrak{p}$ vanishing on the orthogonal of $\mathfrak{a}$ ). The latter expression shows that $c(P,$.$) is a polynomial function on \mathfrak{a}^{*}$ having the same highest order terms as $P(i \lambda)$ (since $\left.e_{\infty}(0)=1\right)$. Because $\varphi_{w \cdot \lambda}=\varphi_{\lambda}$ for $w \in W$ it is $W$-invariant.

Besides $(\widetilde{P} \circ \widetilde{Q}) \widetilde{\psi}_{\lambda}=c(P, \lambda) c(Q, \lambda) \widetilde{\psi}_{\lambda}$ for $P, Q \in \mathbb{D}(\mathfrak{p})^{K}$, and Theorem 3.9 implies

$$
c(P \times Q, \lambda)=c(P, \lambda) c(Q, \lambda) .
$$

The eigenvalues $c(P, \lambda)$ are also given by Harish-Chandra's isomorphism $\Gamma$, which is therefore linked with $e_{\infty}$ by

$$
\Gamma(\widetilde{P})(i \lambda)=c(P, \lambda)=\left(P\left(\partial_{X}+i \lambda\right) e_{\infty}\right)(0)=\left.e_{\infty}\left(\partial_{\xi}\right) P(\xi)\right|_{\xi=i \lambda}
$$


for $P \in \mathbb{D}(\mathfrak{p})^{K}$; the last expression follows from the symmetry of the Fischer product. By Chevalley's theorem the $W$-invariant polynomial $c(P,$.$) on \mathfrak{a}^{*}$ extends to a unique $K$-invariant polynomial on $\mathfrak{p}^{*}$; we still denote it by $c(P,$.$) .$ Knowing that the restriction map $S(\mathfrak{p})^{K} \rightarrow S(\mathfrak{a})^{W}$ and Harish-Chandra's map $\Gamma: \mathbb{D}(S) \rightarrow S(\mathfrak{a})^{W}$ are isomorphisms of algebras, we infer that the map $\gamma: c(P,.) \mapsto \widetilde{P}$ is an isomorphism of the algebra $S(\mathfrak{p})^{K}$ (with standard product) onto $\mathbb{D}(S)$ (with composition of differential operators), that is a generalized Duflo isomorphism. Writing $\gamma(P)=\widetilde{\varepsilon(P)}$ as in Section 3.3.4, the corresponding map $\varepsilon$ is here $c(P,.) \mapsto P$, an isomorphism of $\left(S(\mathfrak{p})^{K}, \cdot\right)$ onto $\left(S(\mathfrak{p})^{K}, \times\right)$. Note that this map may differ from that in 3.3.4 Example 2 however, as the latter depended on a choice of generators of $S(\mathfrak{p})^{K}$. For instance, taking the Laplace operator $\Delta$ of $\mathfrak{p}$ as one of the generators we had $\varepsilon(\Delta)=\Delta$ by the construction in 3.3.4 (Example 3), whereas here

$$
\varepsilon^{-1}(\Delta)(\lambda)=c(\Delta, \lambda)=\widetilde{\Delta} \varphi_{\lambda}(o)=-|\lambda|^{2}-|\rho|^{2}+(n / 12)
$$

by Proposition 3.11 (iv).

More generally, the inverse $\varepsilon^{-1}: P \mapsto c(P,$.$) can be written down explic-$ itly under a slightly stronger assumption on $e$. Assume ${ }^{6}$ that, for any fixed $H \in \mathfrak{a}^{+}$, the limit

$$
e_{\infty}(X, H):=\lim _{t \rightarrow+\infty} e(X, t H)
$$

exists, with uniform convergence for $X$ running in any $K$-orbit in $\mathfrak{p}$, and defines a smooth function of $X \in \mathfrak{p}$. The abridged notation $e_{\infty}(X)$ used up to now disregarded the dependence on $H$. Let $T_{\xi} \in \mathfrak{p}$ correspond to $\xi \in \mathfrak{p}^{*}$ under the identification given by the scalar product on $\mathfrak{p}$; thus $T_{k \cdot \xi}=k \cdot T_{\xi}$ for $k \in K$ and $T_{\lambda} \in \mathfrak{a}$ if $\lambda \in \mathfrak{a}^{*}$ (identified as above with a linear form on $\mathfrak{p}$ vanishing on the orthogonal of $\mathfrak{a})$. For $\xi \in \mathfrak{p}^{* \prime}$, the set of regular elements in $\mathfrak{p}^{*}$, we have $T_{\xi}=k(\xi) \cdot T_{\lambda}$ for some $k(\xi) \in K$ and a unique $T_{\lambda} \in \mathfrak{a}^{+}$. Under our assumption, the $K$-invariance $e\left(X, t T_{\xi}\right)=e\left(k(\xi)^{-1} \cdot X, t T_{\lambda}\right)$ implies the existence of $e_{\infty}\left(X, T_{\xi}\right):=\lim _{t \rightarrow+\infty} e\left(X, t T_{\xi}\right)$ for any $X \in \mathfrak{p}$. Considering

$$
\psi_{\xi}(X):=\int_{K} e^{i\left\langle\xi, k^{-1} \cdot X\right\rangle} e_{\infty}\left(k^{-1} \cdot X, T_{\xi}\right) d k, \xi \in \mathfrak{p}^{* \prime}, X \in \mathfrak{p},
$$

we see that it extends the above definition of $\psi_{\lambda}$ and $\psi_{k \cdot \xi}=\psi_{\xi}$ for $k \in K$. Therefore $\varphi_{\xi}:=\widetilde{\psi}_{\xi}$ is a spherical function on $S$ with eigenvalue

$$
\begin{aligned}
\gamma^{-1}(\widetilde{P})(\xi) & =\varepsilon^{-1}(P)(\xi)=c(P, \xi) \\
& =P \psi_{\xi}(0)=\left.P\left(\partial_{X}+i \xi\right) e_{\infty}\left(X, T_{\xi}\right)\right|_{X=0}
\end{aligned}
$$

for $P \in \mathbb{D}(\mathfrak{p})^{K}$; this gives an explicit formula for the $K$-invariant extension of $c(P, \lambda)$.

\footnotetext{
${ }^{6}$ This assumption holds for special spaces $(G$ complex) and for rank one spaces (Proposition 3.24).
} 
An expression of the operator $\mathcal{T}$ can be obtained similarly. For $u \in \mathcal{D}(\mathfrak{p})^{K}$ the spherical transform of $\widetilde{u} \in \mathcal{D}(S)^{K}$ extends to $\mathfrak{p}^{*}$ as

$$
\begin{aligned}
\mathcal{F}_{S} \widetilde{u}(\xi) & =\int_{S} \widetilde{u}(x) \widetilde{\psi}_{-\xi}(x) d x=\int_{\mathfrak{p}} u(X) \psi_{-\xi}(X) d X \\
& =\int_{\mathfrak{p}} u(X) e_{\infty}\left(X, T_{-\xi}\right) e^{-i\langle\xi, X\rangle} d X
\end{aligned}
$$

This equality holds for $\xi \in \mathfrak{p}^{* \prime}$ but we know the left-hand side extends to an entire function of $\xi \in \mathfrak{p}_{c}^{*}$. Defining as above the operator $\mathcal{T}$ by $\mathcal{F}_{S}=\mathcal{F}_{\mathfrak{p}} \circ \mathcal{T}$ we obtain

$$
\mathcal{T} \widetilde{u}(Y)=\int_{\mathfrak{p}^{*}} \mathcal{F}_{S} \widetilde{u}(\xi) e^{i\langle\xi, Y\rangle} d \xi=\int_{\mathfrak{p} \times \mathfrak{p}^{* \prime}} e^{i\langle\xi, Y-X\rangle} e_{\infty}\left(X, T_{-\xi}\right) u(X) d X d \xi
$$

(the measure $d \xi$ being suitably normalized). The latter is an oscillating integral, defining a pseudo-differential operator of order 0 with symbol $e_{\infty}$. Again

$$
\mathcal{T}\left(\widetilde{u} *_{S} \widetilde{v}\right)=\mathcal{T} \widetilde{u} *_{\mathfrak{p}} \mathcal{T} \widetilde{v}, \mathcal{T}(D \widetilde{u})=\mathcal{T}(D) \mathcal{T} \widetilde{u}
$$

for $u, v \in \mathcal{D}(\mathfrak{p})^{K}, D \in \mathbb{D}(S)$, where $\mathcal{T}(D) \in \mathbb{D}(\mathfrak{p})^{K}$ is defined by $\mathcal{T}(D)(\xi)=$ $\gamma^{-1}(D)(-i \xi)$.

Summing up, under our assumption about $e_{\infty}$, this function allows constructing an operator $\mathcal{T}$ which intertwines the $G$-invariant differential operators in $\mathbb{D}(S)$ with constant coefficients differential operators in $\mathbb{D}(\mathfrak{p})^{K}$ and, more generally, the convolutions products on $S$ and $\mathfrak{p}$.

c. Harish-Chandra's $c$-function. We finally note the following link between $e_{\infty}$ and Harish-Chandra's function $c(\lambda)$. Here again we consider $e_{\infty}$ as a (smooth) function on $\mathfrak{p}$ only, forgetting its dependence upon the second variable which is not needed any more. According to a fundamental theorem of spherical harmonic analysis, the function $\mu(\lambda):=|c(\lambda)|^{-2}$ is $W$-invariant and tempered on $\mathfrak{a}^{*}$, and gives the inversion formula of the spherical transform

$$
u(0)=\widetilde{u}(o)=\int_{\mathfrak{a}^{*}} \mathcal{F}_{S} \widetilde{u}(\lambda) \mu(\lambda) d \lambda=\left\langle\mu(\lambda), \mathcal{F}_{\mathfrak{p}}\left(e_{\infty} u\right)(\lambda)\right\rangle
$$

for $u \in \mathcal{D}(\mathfrak{p})^{K}$; the latter equality follows from (3.41). Writing as above $\mathfrak{p}=\mathfrak{a} \oplus \mathfrak{q}$ and $X=H+Y$ the corresponding decomposition we have

$$
\mathcal{F}_{\mathfrak{p}}\left(e_{\infty} u\right)(\lambda)=\int_{\mathfrak{a}} e^{-i\langle\lambda, H\rangle} d H \int_{\mathfrak{q}}\left(e_{\infty} u\right)(H+Y) d Y .
$$

Thus, using the Fourier transform of the tempered distribution $\mu$,

$$
u(0)=\left\langle\left(\mathcal{F}_{\mathfrak{a}^{*}} \mu\right)(H), \int_{\mathfrak{q}}\left(e_{\infty} u\right)(H+Y) d Y\right\rangle
$$


for any $K$-invariant test function $u$. To write it more concisely let $\pi^{*} T=$ $T \circ \pi \in \mathcal{D}^{\prime}(\mathfrak{p})$ denote the pullback of a distribution $T \in \mathcal{D}^{\prime}(\mathfrak{a})$ by the submersion $\pi: \mathfrak{p} \rightarrow \mathfrak{a}$, that is $\langle T \circ \pi(X), f(X)\rangle=\langle T(H) \otimes 1(Y), f(H+Y)\rangle$, and $U_{K}$ the $K$-invariant mean of $U \in \mathcal{D}^{\prime}(\mathfrak{p})$, that is $\left\langle U_{K}(X), f(X)\right\rangle=$ $\left\langle U(X), \int_{K} f(k \cdot X) d k\right\rangle$. We obtain the following relation between $e_{\infty}$ and the Plancherel measure $\mu$ :

$$
\left(e_{\infty} \cdot\left(\left(\mathcal{F}_{\mathfrak{a}^{*}} \mu\right) \circ \pi\right)\right)_{K}=\delta_{0},
$$

the Dirac measure at the origin of $\mathfrak{p}$.

d. Remark. Let $\varphi_{i \xi}^{0}(X)=\int_{K} e^{i<\xi, k \cdot X>} d k$, with $\xi \in \mathfrak{p}^{*}, X \in \mathfrak{p}$, be the generalized Bessel function already introduced. With $\mathcal{T}=\mathcal{F}_{\mathfrak{p}}^{-1} \circ \mathcal{F}_{S}$ as above we have the following formal relations

$$
\begin{aligned}
{ }^{t} \mathcal{T} \varphi_{i \xi}^{0} & =\varphi_{\xi}, \xi \in \mathfrak{p}^{*}, \\
\left(\mathcal{T}^{-1} \varphi_{i \xi}^{0}\right)(x) & =\left(\mathcal{T}^{-1} \varphi_{i \xi}^{0}\right)(o) \varphi_{\xi}(x), x \in G / K,
\end{aligned}
$$

and $\left(\mathcal{T}^{-1} \varphi_{i \xi}^{0}\right)(o) d \xi$ is the Plancherel measure in the inversion formula for the spherical transform.

Indeed, for any $K$-invariant test function $f$ on $S$,

$$
\begin{aligned}
\int_{\mathfrak{p}} \mathcal{T} f(X) \varphi_{-i \xi}^{0}(X) d X & =\int_{\mathfrak{p}} \mathcal{T} f(X) e^{-i\langle\xi, X\rangle} d X=\mathcal{F}_{\mathfrak{p}} \mathcal{T} f(\xi) \\
& =\mathcal{F}_{S} f(\xi)=\int_{S} f(x) \varphi_{-\xi}(x) d x
\end{aligned}
$$

whence the first result using the formal transpose ${ }^{t} \mathcal{T}$. Moreover

$$
\mathcal{T} f(X)=\mathcal{F}_{\mathfrak{p}}^{-1} \circ \mathcal{F}_{S} f(X)=\int_{\mathfrak{p}^{*}} \mathcal{F}_{S} f(\xi) e^{i\langle\xi, X\rangle} d \xi=\int_{\mathfrak{p}^{*}} \mathcal{F}_{S} f(\xi) \varphi_{i \xi}^{0}(X) d \xi
$$

and, applying $\mathcal{T}^{-1}$ (formally) under the integral sign,

$$
f(x)=\int_{\mathfrak{p}^{*}} \mathcal{F}_{S} f(\xi)\left(\mathcal{T}^{-1} \varphi_{i \xi}^{0}\right)(x) d \xi .
$$

For $x=o$ this shows that $\left(\mathcal{T}^{-1} \varphi_{i \xi}^{0}\right)(o) d \xi$ is the Plancherel measure.

Our second formal relation now follows from a classical calculation. We note that, for $g \in G$ and $k \in K$,

$$
\int_{S} f(g k x) \varphi_{-\xi}(x) d x=\int_{S} f(x) \varphi_{-\xi}\left(g^{-1} k x\right) d x,
$$

as given by the change $x \mapsto k^{-1} g k x$. Let $f_{g}(x):=\int_{K} f(g k x) d k$. Integrating with respect to $k$ we infer

$$
\mathcal{F}_{S} f_{g}(\xi)=\mathcal{F}_{S} f(\xi) \varphi_{-\xi}\left(g^{-1} K\right)=\mathcal{F}_{S} f(\xi) \varphi_{\xi}(g K)
$$


from the functional equation of spherical functions and a symmetry property of $\varphi_{\xi}$, known for $\xi \in \mathfrak{a}^{*}$ ([28] p. 419) and extended here to $\mathfrak{p}^{*}$. The inversion formula (3.43) at the origin gives

$$
\begin{aligned}
f(g K) & =f_{g}(o)=\int_{\mathfrak{p}^{*}} \mathcal{F}_{S} f_{g}(\xi)\left(\mathcal{T}^{-1} \varphi_{i \xi}^{0}\right)(o) d \xi \\
& =\int_{\mathfrak{p}^{*}} \mathcal{F}_{S} f(\xi)\left(\mathcal{T}^{-1} \varphi_{i \xi}^{0}\right)(o) \varphi_{\xi}(g K) d \xi
\end{aligned}
$$

and our claim follows by comparison with (3.43).

\subsection{An $e$-Function for Symmetric Spaces of Rank One}

In this section we consider a Riemannian symmetric space $G / K$ of the noncompact type and of rank one, that is one of the hyperbolic spaces (real, complex, quaternionic or Cayley). Standard semisimple notation will be used throughout, as defined in Helgason's books [27][28]; see Section 0.5. Let $\alpha$, and possibly $2 \alpha$, be the positive roots with respective multiplicities $p \geq 1$ and $q \geq 0$; thus $\operatorname{dim} G / K=n=p+q+1$. On the Lie algebra $\mathfrak{g}$ it will be convenient to use the norm

$$
\|X\|:=\left(-\frac{1}{2(p+4 q)} B(X, \theta X)\right)^{1 / 2}
$$

where $B$ is the Killing form and $\theta$ the Cartan involution, so that $H \in \mathfrak{a}$ and $\alpha(H)=1$ imply $\|H\|=1$ by the root space decomposition of ad $H$. We now construct an explicit $e$-function ${ }^{7}$.

Theorem 3.23 Let $G / K$ be a Riemannian symmetric space of the noncompact type and of rank one. Let $\sigma(t):=\operatorname{sh} t / t, x=\|X\|, y=\|Y\|$, $z=\|X+Y\|$ for $X, Y \in \mathfrak{p}$ and

$$
\begin{aligned}
& A(x, y, z)=\frac{1}{\sigma(x) \sigma(y) \sigma(z)} \times \\
& \quad \times \sigma\left(\frac{x+y+z}{2}\right) \sigma\left(\frac{x+y-z}{2}\right) \sigma\left(\frac{x-y+z}{2}\right) \sigma\left(\frac{-x+y+z}{2}\right) \\
& B(x, y, z)=\frac{1}{\operatorname{ch} x \operatorname{ch} y \operatorname{ch} z} \times \\
& \quad \times \operatorname{sh}\left(\frac{x+y+z}{2}\right) \operatorname{sh}\left(\frac{x+y-z}{2}\right) \operatorname{sh}\left(\frac{x-y+z}{2}\right) \operatorname{sh}\left(\frac{-x+y+z}{2}\right)
\end{aligned}
$$

${ }^{7}$ This theorem is (unpublished) joint work with Mogens Flensted-Jensen. 
Then

$$
e(X, Y):=A(x, y, z)^{(n-3) / 2}{ }_{2} F_{1}\left(1-\frac{q}{2}, \frac{q}{2} ; \frac{n-1}{2} ; B(x, y, z)\right)
$$

is an e-function of $G / K$ associated with $j=J^{1 / 2}$ (the square root of the Jacobian of Exp), analytic on $\mathfrak{p} \times \mathfrak{p}$.

Remarks. (a) The hypergeometric factor ${ }_{2} F_{1}$ is identically 1 if $q=0$, that is when $G / K$ is a real hyperbolic space $H^{n}(\mathbb{R})$. Among them $H^{3}(\mathbb{R})$ is special; this results from Proposition 3.7 too, since $H^{3}(\mathbb{R})=S L(2, \mathbb{C}) / S U(2)$.

(b) Note that $e(X, Y)$ is here globally defined and invariant under all permutations of the three variables $x, y, z$. Further properties of $e$ will be given in Proposition 3.24.

(c) $A$ and $B$ may also be written as

$$
\begin{aligned}
& A(x, y, z)=\frac{4 x y z}{\operatorname{sh} x \operatorname{sh} y \operatorname{sh} z} \frac{\operatorname{ch}(x+y)-\operatorname{ch} z}{(x+y)^{2}-z^{2}} \frac{\operatorname{ch} z-\operatorname{ch}(x-y)}{z^{2}-(x-y)^{2}} \\
& B(x, y, z)=\frac{(\operatorname{ch}(x+y)-\operatorname{ch} z)(\operatorname{ch} z-\operatorname{ch}(x-y))}{4 \operatorname{ch} x \operatorname{ch} y \operatorname{ch} z}
\end{aligned}
$$

Example. For $H^{n}(\mathbb{R})$ the function $\log e=\frac{n-3}{2} \log A$ expands as

$$
\log e(X, Y)=\frac{3-n}{120}\left(\|X\|^{2}\|Y\|^{2}-(X \cdot Y)^{2}\right)+\cdots
$$

in a neighborhhod of the origin, where $\cdots$ have order $\geq 6$ with respect to $(X, Y)$ and

$$
X \cdot Y=\frac{1}{2(n-1)} B(X, Y)
$$

is the scalar product on $\mathfrak{p}$ associated with the norm (3.44). Proposition 4.31 below gives a similar expansion for arbitrary symmetric spaces.

Indeed $\log \sigma(t)=\left(t^{2} / 6\right)-\left(t^{4} / 180\right)+O\left(t^{6}\right)$, the second order terms in $\log A$ cancel out and it is easily checked that

$$
\begin{aligned}
\log A & =\frac{1}{240}\left(\left(x^{2}+y^{2}-z^{2}\right)^{2}-4 x^{2} y^{2}\right)+\cdots \\
& =-\frac{1}{60}\left(\|X\|^{2}\|Y\|^{2}-(X \cdot Y)^{2}\right)+\cdots
\end{aligned}
$$

\subsubsection{Outline of the Proof}

Not surprisingly the proof of Theorem 3.23 is a bit technical; we only give here a brief sketch of its main steps and refer to Appendix B for full details. What we are looking for is an integral formula of the form

$$
\int_{K} f(Z(X, k \cdot Y)) d k=\int_{K} f(X+k \cdot Y) D(X, k \cdot Y) d k,
$$


valid for any $K$-invariant continuous function $f$ on $\mathfrak{p}$ and any $X, Y \in \mathfrak{p}$, where the factor $D$ is to be explicitly computed and, as above, $Z(X, Y) \in \mathfrak{p}$ is defined by $e^{Z(X, Y)} K=e^{X} e^{Y} K$. In view of Proposition 3.16 (ii) this will exhibit an $e$-function for $G / K$.

By the polar decomposition of $X \in \mathfrak{p}$ we have $X=k_{X} \cdot(\|X\| H)$ for some $k_{X} \in K$, since our space has rank one and $\|H\|=1$. Letting $\varphi(t)=f(t H)$ it is therefore sufficient to prove that

$$
\int_{K} \varphi(\|Z(X, k \cdot Y)\|) d k=\int_{K} \varphi(\|X+k \cdot Y\|) D(X, k \cdot Y) d k
$$

for any continuous function $\varphi$ on $[0, \infty[$. This will be achieved first in the special case $X, Y \in \mathfrak{a}^{+}$: taking $X=x H$ and $Y=y H$ with $x, y>0$ we will prove that

$$
\begin{aligned}
\int_{K} \varphi(\|x H+k \cdot y H\|) d k & =\int_{|x-y|}^{x+y} \varphi(z) a(x, y, z) d z \\
\int_{K} \varphi(\|Z(x H, k \cdot y H)\|) d k & =\int_{|x-y|}^{x+y} \varphi(z) b(x, y, z) d z
\end{aligned}
$$

with $a, b$ given by explicit formulas. Replacing $\varphi(z)$ by $\varphi(z) b(x, y, z) / a(x, y, z)$ in (3.46) it will follow that

$$
\begin{aligned}
\int_{K} \varphi(\|Z(x H, k \cdot y H)\|) d k & =\int_{|x-y|}^{x+y} \varphi(z) \frac{b}{a}(x, y, z) a(x, y, z) d z \\
& =\int_{K} \varphi(\|x H+k \cdot y H\|) \frac{b}{a}(x, y,\|x H+k \cdot y H\|) d k
\end{aligned}
$$

which is $(3.45)$ for $X, Y \in \mathfrak{a}^{+}$with

$$
D(X, Y)=\frac{b}{a}(\|X\|,\|Y\|,\|X+Y\|) .
$$

The general case $X, Y \in \mathfrak{p}$ follows by $K$-invariance, writing $X=k_{X} \cdot x H$ and $Y=k_{Y} \cdot y H$ and changing $k$ into $k_{X}^{-1} k k_{Y}$ in both integrals over $K$. This will finally give the $e$-function

$$
e(X, Y)=\frac{j(X) j(Y)}{j(X+Y)} \frac{b}{a}(\|X\|,\|Y\|,\|X+Y\|) .
$$

The main point is therefore to prove (3.46) and (3.47). The former is elementary (Lemma B.2). For the latter (Lemma B.5) let $g=k(g) e^{H(g)} n(g)$ be the Iwasawa decomposition of $g \in G$ according to $G=K A N$, let $M$ be the centralizer of $A$ in $K$, and $\bar{N}=\theta N$ where $\theta$ is the Cartan involution. The proof relies on the classical integral formula (valid for arbitrary rank, see [28] p. 198):

$$
\int_{K} f(k) d k=\int_{\bar{N}} f(k(\bar{n})) e^{-2\langle\rho, H(\bar{n})\rangle} d \bar{n},
$$


if $f$ is any $M$-bi-invariant continuous function on $K, \rho$ is the half-sum of positive roots counted with mutiplicities and the Haar measure $d \bar{n}$ is suitably normalized. In the rank one case $A$ is one-dimensional and the right-hand side can be computed by $S U(2,1)$-reduction (Lemma B.4). Applying this to the $M$-bi-invariant function $f(k)=\varphi(\|Z(x H, k \cdot y H)\|)$, we shall finally obtain (3.47).

\subsubsection{Properties of the Rank One $e$-Function}

The main properties of the function obtained in the rank one case can be easily read from the explicit formula in Theorem 3.23. Let us emphasize their similarity with the properties of the $e$-function constructed in the next chapter by a completely different method (cf. Section 4.4). Proposition 3.25 explains this, for real hyperbolic spaces at least.

Proposition 3.24 Let $G / K$ be a Riemannian symmetric space of the noncompact type and of rank one. The e-function of Theorem 3.23 has the following properties.

(i) It is analytic and strictly positive on $\mathfrak{p} \times \mathfrak{p}$.

(ii) For any $k \in K$ and $X, Y \in \mathfrak{p}$,

$$
e(k \cdot X, k \cdot Y)=e(X, Y)=e(-X,-Y)=e(Y, X)=e(X, Z)
$$

whenever $Z \in \mathfrak{p}$ and $X+Y+Z=0$.

(iii) $\partial_{Y} e(X, 0)=0$ and $\partial_{X} e(0, Y)=0$.

(iv) $e(X, Y)=1$ whenever $[X, Y]=0$.

(v) Let $X, Y \in \mathfrak{p}$ with $Y \neq 0, x=\|X\|$ and $x^{\prime}=(X \cdot Y) /\|Y\|$, where $\|X\|$ is the norm (3.44) and $X \cdot Y$ the corresponding scalar product on $\mathfrak{p}$. Then

$$
\begin{aligned}
& \lim _{t \rightarrow \pm \infty} e(X, t Y)= \\
& \quad=\left(2 \frac{x}{\operatorname{sh} x} \frac{\operatorname{ch} x-\operatorname{ch} x^{\prime}}{x^{2}-x^{\prime 2}}\right)^{(n-3) / 2}{ }_{2} F_{1}\left(1-\frac{q}{2}, \frac{q}{2} ; \frac{n-1}{2} ; \frac{\operatorname{ch} x-\operatorname{ch} x^{\prime}}{2 \operatorname{ch} x}\right),
\end{aligned}
$$

uniformly for fixed $Y$ and $X$ running in an arbitrary compact subset of $\mathfrak{p}$. The right-hand side is an analytic function of $(X, Y)$ on $\mathfrak{p} \times(\mathfrak{p} \backslash\{0\})$.

Proof. We retain the notation $A, B$ of Theorem 3.23 and Remark (c) immediately after. They are even analytic functions of $x, y, z$, therefore analytic in $(X, Y) \in \mathfrak{p} \times \mathfrak{p}$.

(i) In view of the expressions (B.6)(B.7) of $B$ in Appendix B we have $0 \leq$ $B(x, y, z)<1 / 2$ for $|x-y| \leq z \leq x+y$ and the hypergeometric factor of $e$ is analytic with respect to $(X, Y) \in \mathfrak{p} \times \mathfrak{p}$. It is strictly positive too (see the end of proof of Lemma B.5). Besides $A(x, y, z)$ is strictly positive for $(x, y, z) \in \mathbb{R}^{3}$. 
(ii) is easy. By Remark (b) following Theorem $3.23 e$ is a symmetric function of $x, y, z$, hence

$$
\begin{aligned}
e(X, Y) & =f(\|X\|,\|Y\|,\|X+Y\|)=f(\|X\|,\|-X-Y\|,\|-Y\|) \\
& =e(X,-X-Y) .
\end{aligned}
$$

(iii) Differentiating $e(X, Y)=e(-X, X+Y)$ with respect to $X$ at $X=0$ we obtain

$$
\partial_{X} e(0, Y)=-\partial_{X} e(0, Y)+\partial_{Y} e(0, Y) .
$$

But $e(0, Y)=1$ hence $\partial_{Y} e(0, Y)=0$ and we infer $\partial_{X} e(0, Y)=0$. Then $\partial_{Y} e(X, 0)=0$ because $e(X, Y)=e(Y, X)$.

(iv) By $K$-invariance it suffices to consider the special case $X=x H, x \geq 0$. If $x=0$ we have $e(0, Y)=1$. If $x>0$ the assumption $[X, Y]=0$ and $Y \in \mathfrak{p}$ implies $Y=t H$ for some $t \in \mathbb{R}$, therefore $y= \pm t, z= \pm(x+t)$ and it is easily checked that $A=1$ and $B=0$ whence $e(X, Y)=1$.

(v) Let $Y \in \mathfrak{p}$ with $\|Y\|=y>0$. Since $e(X, t Y)=e(-X,-t Y)$ we may assume $t>0$. First

$$
z=\|X+t Y\|=t y+x^{\prime}+r \text { with } 0 \leq r \leq x^{2} / 2 t y ;
$$

indeed $z^{2}=x^{2}+t^{2} y^{2}+2 t x^{\prime} y$ and $2 t y\left(z-t y-x^{\prime}\right)=x^{2}-(t y-z)^{2}$ lies between 0 and $x^{2}$. We can now study the behavior of the factors of $A(x, t y, z)$ as $t \rightarrow+\infty$ and $X$ remains in a compact subset of $\mathfrak{p}$, say $\|X\|=x \leq C$. First

$\frac{1}{\sigma(x)} \sigma\left(\frac{x-t y+z}{2}\right) \sigma\left(\frac{x+t y-z}{2}\right)=\frac{1}{\sigma(x)} \sigma\left(\frac{x+x^{\prime}+r}{2}\right) \sigma\left(\frac{x-x^{\prime}-r}{2}\right)$

tends to $\sigma\left(\frac{x+x^{\prime}}{2}\right) \sigma\left(\frac{x-x^{\prime}}{2}\right) / \sigma(x)$, uniformly for $\|X\| \leq C$. Furthermore these factors remain uniformly bounded for $\|X\| \leq C$ and $t \geq 1 / 2 y$ because $\left|x \pm x^{\prime} \pm r\right| \leq 2 x+x^{2} \leq 2 C+C^{2}$ and $\sigma(x) \geq 1$. The remaining factors of $A$ are

$$
\begin{gathered}
\frac{\sigma\left(t y+\frac{x^{\prime}+x+r}{2}\right) \sigma\left(t y+\frac{x^{\prime}-x+r}{2}\right)}{\sigma(t y) \sigma\left(t y+x^{\prime}+r\right)}=A_{1} A_{2}, \\
\text { with } A_{1}:=\left(1-\frac{\operatorname{ch} x}{\operatorname{ch}\left(2 t y+x^{\prime}+r\right)}\right)\left(1-\frac{\operatorname{ch}\left(x^{\prime}+r\right)}{\operatorname{ch}\left(2 t y+x^{\prime}+r\right)}\right)^{-1} \\
A_{2}:=\left(1+\frac{x^{\prime}+r}{t y}\right)\left(1+\frac{x^{\prime}+x+r}{2 t y}\right)^{-1}\left(1+\frac{x^{\prime}-x+r}{2 t y}\right)^{-1} .
\end{gathered}
$$

No problem arises from the denominators for $t$ large enough (independently of $X$ ). Thus $A_{1}$ and $A_{2}$ converge to 1 as $t \rightarrow+\infty$, uniformly for $\|X\| \leq C$. Gathering all factors we see that $A(x, t y, z)$ tends to $\sigma\left(\frac{x+x^{\prime}}{2}\right) \sigma\left(\frac{x-x^{\prime}}{2}\right) / \sigma(x)$, uniformly for $\|X\| \leq C$, and remains bounded. 
A similar argument shows that $B(x, t y, z)$ tends to $\operatorname{sh}\left(\frac{x+x^{\prime}}{2}\right) \operatorname{sh}\left(\frac{x-x^{\prime}}{2}\right) / \operatorname{ch} x$, uniformly for $\|X\| \leq C$, and remains bounded. This completes the proof of the proposition.

We end this section with a discussion of uniqueness of $e$-functions.

Proposition 3.25 (i) On the real hyperbolic space $H^{n}(\mathbb{R}), n \geq 3$, for any $j$ there exists a unique $e$-function associated to $j$. For $j=J^{1 / 2}$ it is

$$
e(X, Y)=A(x, y, z)^{(n-3) / 2}
$$

in the notation of Theorem 3.23, with $X, Y \in \mathfrak{p}, x=\|X\|, y=\|Y\|, z=$ $\|X+Y\|$.

(ii) This formula also holds for $n=2$ but e is not unique in this case.

Proof. Existence for $n \geq 2$. For $j=J^{1 / 2}$ the result follows from Theorem 3.23 with $q=0$. Any other function $j$ may be written as $j(X)=\lambda(x) J^{1 / 2}(X)$ with $x=\|X\|$. By Remark $(a)$ in 3.1.2, multiplication of the previous function $e$ by $\lambda(x) \lambda(y) / \lambda(z)$ gives an $e$-function associated to $j$.

Uniqueness for $n \geq 3$. Let $\varepsilon=e_{1}-e_{2}$ where $e_{1}$ and $e_{2}$ are two $e$-functions of $G / K$ associated to the same $j$. By Proposition 3.16 (ii) with $j^{-1} f$ instead of $f$ we have

$$
\int_{K} f(X+k \cdot Y) \varepsilon(X, k \cdot Y) d k=0
$$

for any $K$-invariant smooth function $f$ on $\mathfrak{p}$ and any $X, Y \in \mathfrak{p}$. The uniqueness of $e$ for $G / K=H^{n}(\mathbb{R})$ will stem from a Cartan type decomposition $K=M L M$, where $L$ is here a one-dimensional subgroup, allowing to replace (3.49) by an integral with respect to a single variable and finally conclude that $\varepsilon=0$.

Here $H^{n}(\mathbb{R})=G / K$ with $G=S O_{0}(n, 1), K=S O(n) \times\{1\}$ and, as in the proof of Lemma B.3, we identify an element $X$ of $\mathfrak{p}$ with $X=\left(x_{1}, \ldots, x_{n}\right) \in \mathbb{R}^{n}$ for short. The adjoint action of $K$ on $\mathfrak{p}$ is then the natural action of $S O(n)$ on $\mathbb{R}^{n}$. Taking the unit vector $H=(1,0, \ldots, 0)$ as a basis of $\mathfrak{a}$, the stabilizer of $H$ in $K$ is $M=\{1\} \times S O(n-1) \times\{1\}$. The group $M$ rotates the $n-1$ last components of vectors in $\mathbb{R}^{n}$ therefore, given any $k \in K$, there exist $m \in M$ and $\theta \in \mathbb{R}$ such that

$$
m k \cdot H=(\cos \theta, \sin \theta, 0, \ldots, 0)=l_{\theta} \cdot H
$$

where $l_{\theta} \in L$, the one-dimensional subgroup of rotations in the $\left(x_{1}, x_{2}\right)$ plane. This implies $k=m^{-1} l_{\theta} m^{\prime}$ for some $m, m^{\prime} \in M$ and $l_{\theta} \in L$; thus $K=M L M$. For $n \geq 3, M$ is a non-trivial group and we may even choose $m \in M$ such that $\sin \theta \geq 0$ in $(3.50)$, whence $l_{\theta} \cdot H=\left(t, \sqrt{1-t^{2}}, 0, \ldots, 0\right)$ with $t=\cos \theta=\pi\left(l_{\theta} \cdot H\right)=\pi(k \cdot H), \pi$ denoting the orthogonal projection $\mathfrak{p} \rightarrow \mathfrak{a}$. Going back to (3.49), we take $X=x H, Y=y H$ with $x, y>0$ at first. The invariances allow replacing $k$ by $l_{\theta}$ in $f(x H+y k \cdot H) \varepsilon(x H, y k \cdot H)$, which 
therefore only depends on the a-component $t$ (and $x, y$ ). Now Lemma B.1 and the proof of Lemma B.2 in Appendix B lead to

$\int_{K} f(x H+k \cdot y H) \varepsilon(x H, k \cdot y H) d k=\int_{|x-y|}^{x+y} f(z H) \varepsilon\left(x H, l_{\theta} \cdot y H\right) a(x, y, z) d z$,

with $z$ linked to $\theta \in[0, \pi]$ by $z=\left\|x H+l_{\theta} \cdot y H\right\|=\sqrt{x^{2}+y^{2}+2 x y t}$ and $a$ as in Lemma B.2. The vanishing of this integral for any ( $K$-invariant) $f$ implies $\varepsilon\left(x H, l_{\theta} \cdot y H\right)=0$ for $\left.\theta \in\right] 0, \pi[$ and $x, y>0$, whence $\varepsilon(x H, k \cdot y H)=0$ for $k \in K$ and finally $\varepsilon(X, Y)=0$ for all $X, Y \in \mathfrak{p}$ by $K$-invariance and continuity, as claimed.

Non-uniqueness for $n=2$. The above argument breaks down for $n=2$. In this case, with $X=\left(x_{1}, x_{2}\right)$ and $Y=\left(y_{1}, y_{2}\right)$ as above, let $\varepsilon(X, Y):=$ $\varphi\left(x_{1} y_{2}-x_{2} y_{1}\right)$ where $\varphi$ is an arbitrary analytic odd function on $\mathbb{R}$. Then $\varepsilon(k \cdot X, k \cdot Y)=\varepsilon(X, Y)$ and we claim that the integral

$$
\int_{0}^{2 \pi} f\left(X+k_{\theta} \cdot Y\right) \varepsilon\left(X, k_{\theta} \cdot Y\right) \frac{d \theta}{2 \pi}
$$

with $k_{\theta}=\left(\begin{array}{cc}\cos \theta & -\sin \theta \\ \sin \theta & \cos \theta\end{array}\right) \in K$, vanishes for any $K$-invariant function $f$. Indeed, fixing $X=x H$ and $Y=y H \in \mathfrak{a}$ (which will suffice in view of the $K$-invariance of $f$ and $\varepsilon$ ), we see that $f\left(X+k_{\theta} \cdot Y\right)$ is a function of $\cos \theta$ and $\varepsilon\left(X, k_{\theta} \cdot Y\right)=\varphi(x y \sin \theta)$ is an odd function of $\sin \theta$, hence our claim. Thus, if $e$ is an $e$-function of $H^{2}(\mathbb{R}), e+\varepsilon$ is a new one.

\subsubsection{Application to Spherical Functions}

As above let $H \in \mathfrak{a}$ be defined by $\alpha(H)=1$ (so that $\|H\|=1$ ) and let $\pi: \mathfrak{p} \rightarrow \mathfrak{a}$ denote the orthogonal projection. Applying Proposition 3.24 $(v)$ with $X \in \mathfrak{p}$ and $Y=H$, we obtain $x^{\prime}=\pi(X)$ (under the obvious identification of $\mathfrak{a}=\mathbb{R} H$ with $\mathbb{R}$ ) and

$$
\begin{array}{r}
e_{\infty}(X)=\left(2 \frac{\|X\|}{\operatorname{sh}\|X\|} \frac{\operatorname{ch}\|X\|-\operatorname{ch} \pi(X)}{\|X\|^{2}-\pi(X)^{2}}\right) \stackrel{(n-3) / 2}{ }{ }^{(x} \times \\
\quad \times{ }_{2} F_{1}\left(1-\frac{q}{2}, \frac{q}{2} ; \frac{n-1}{2} ; \frac{\operatorname{ch}\|X\|-\operatorname{ch} \pi(X)}{2 \operatorname{ch}\|X\|}\right),
\end{array}
$$

analytic on $\mathfrak{p}$, in the notation of Theorem 3.18 which applies to rank one spaces. Thus, identifying $\mathfrak{a}^{*}$ with $\mathbb{R}$ too,

$$
J(t H)^{1 / 2} \varphi_{\lambda}(\operatorname{Exp} t H)=\int_{K} e^{i t \lambda \pi(k \cdot H)} e_{\infty}(t k \cdot H) d k
$$

for all $t, \lambda \in \mathbb{R}$, that is

$$
\left(\frac{\operatorname{sh} t}{t}\right)^{(n-1) / 2}(\operatorname{ch} t)^{q / 2} \varphi_{\lambda}(\operatorname{Exp} t H)=\int_{K} f(\pi(k \cdot H)) d k
$$


with

$f(u):=e^{i t \lambda u}\left(\frac{2}{t \operatorname{sh} t} \frac{\operatorname{ch} t-\operatorname{ch} t u}{1-u^{2}}\right)^{(n-3) / 2}{ }_{2} F_{1}\left(1-\frac{q}{2}, \frac{q}{2} ; \frac{n-1}{2} ; \frac{\operatorname{ch} t-\operatorname{ch} t u}{2 \operatorname{ch} t}\right)$.

The integral over $K$ can be expressed by means of Lemma B.1 in the Appendix and it follows easily (with $s=t u$ ) that

$$
\begin{aligned}
& \frac{\Gamma\left(\frac{n-1}{2}\right) \Gamma\left(\frac{1}{2}\right)}{2^{(n-1) / 2} \Gamma\left(\frac{n}{2}\right)}(\operatorname{sh} t)^{n-2}(\operatorname{ch} t)^{q / 2} \varphi_{\lambda}(\operatorname{Exp} t H)= \\
& \quad=\int_{0}^{t} \cos \lambda s(\operatorname{ch} t-\operatorname{ch} s)^{(n-3) / 2}{ }_{2} F_{1}\left(1-\frac{q}{2}, \frac{q}{2} ; \frac{n-1}{2} ; \frac{\operatorname{ch} t-\operatorname{ch} s}{2 \operatorname{ch} t}\right) d s .
\end{aligned}
$$

Obtained here by means of $e_{\infty}$, this is a formula proved by Koornwinder ([33] p. 149 or [34] p. 47-48) for all Jacobi functions.

\subsection{Extension to Line Bundles}

Given a symmetric space $S=G / H$ and a character $\chi$ of $H$ we consider the line bundle $L_{\chi}$, keeping to the notation of Section 2.2. Elements of $L_{\chi}$ are denoted by $(x, z)$ with $x \in G, z \in \mathbb{C}$. Given $j$ as in Definition 3.2, the transfer of a smooth function $f$ on $\mathfrak{s}^{\prime}$ is now the section $\widetilde{f}$ of $L_{\chi}$ above $\operatorname{Exp} \mathfrak{s}^{\prime}$ defined by

$$
\widetilde{f}(\operatorname{Exp} X)=\underline{\left(e^{X}, j(X)^{-1} f(X)\right) .}
$$

For a distribution $u$ on $\mathfrak{s}^{\prime}$ the distribution $\widetilde{u}$ on $L_{\chi}$ is defined by

$$
\langle\widetilde{u}, \tilde{f}\rangle=\langle u, f\rangle
$$

for all $f \in \mathcal{D}\left(\mathfrak{s}^{\prime}\right)$. The transfer map preserves $H$-invariance. When restricting to $H$-invariant distributions supported at the origin, that is $u={ }^{t} P \delta_{0}$ with $P \in \mathbb{D}(\mathfrak{s})^{H}$, we obtain an order preserving linear isomorphism $P \mapsto \widetilde{P}$ of $\mathbb{D}(\mathfrak{s})^{H}$ onto $\mathbb{D}\left(L_{\chi}\right)$. If $s(x H)=(x, f(x))$ is a smooth section we have

$$
(\widetilde{P} s)(x H)=\underline{(x, g(x))} \text { with } g(x)=P\left(\partial_{X}\right)\left(j(X) f\left(x e^{X}\right)\right)_{X=0} .
$$

Definition 3.26 An e-function of $L_{\chi}$ is an analytic function $e_{\chi}: \Omega \rightarrow \mathbb{R}$ on an open neighborhood $\Omega$ of $(0,0)$ in $\mathfrak{s}^{\prime} \times \mathfrak{s}^{\prime}$ such that:

(i) for all $h \in H$ and $(X, Y) \in \Omega$ one has $(h \cdot X, h \cdot Y) \in \Omega$ and $e_{\chi}(h \cdot X, h \cdot Y)=$ $e_{\chi}(X, Y)$

(ii) for any $H$-invariant distributions $u, v$ and any smooth function $f$ on open neighborhoods of the origin in $\mathfrak{s}^{\prime}$ (with suitable supports),

$$
\langle\widetilde{u} * \widetilde{v}, \tilde{f}\rangle=\left\langle u(X) \otimes v(Y), e_{\chi}(X, Y) f(X+Y)\right\rangle .
$$


The product $*$ is here the convolution on $L_{\chi}$ defined in Section 2.2.2. The existence of such a function on some suitably chosen $\Omega$ and its link with an $e$-function of $S$ will be given by Theorem 4.25. We now derive some consequences of this definition. Theorems 3.8 and 3.9 generalize as follows, with the same proof.

Theorem 3.27 Let $e_{\chi}: \Omega \rightarrow \mathbb{R}$ be an e-function of the line bundle $L_{\chi}$ and $\Omega_{1}=\{X \in \mathfrak{s} \mid(X, 0) \in \Omega\}$.

(i) For $P \in \mathbb{D}(\mathfrak{s})^{H}$ the equation

$$
P_{\chi}\left(X, \partial_{X}\right) f(X):=\left.P\left(\partial_{Y}\right)\left(e_{\chi}(X, Y) f(X+Y)\right)\right|_{Y=0},
$$

$f \in C^{\infty}\left(\Omega_{1}\right)$, defines an $H$-invariant differential operator $P_{\chi}$ with analytic coefficients on $\Omega_{1}$ and symbol $P_{\chi}(X, \xi)=e_{\chi}\left(X, \partial_{\xi}\right) P(\xi)$. For any $H$-invariant distribution $u$ on $\Omega_{1}$

$$
{ }^{t} \widetilde{P} \widetilde{u}=\left({ }^{t} P_{\chi}\left(X, \partial_{X}\right) u\right) \sim \text { on } \operatorname{Exp} \Omega_{1} .
$$

(ii) If $H$ is compact and $f$ is any $H$-invariant smooth function on $\Omega_{1}$

$$
\widetilde{P} \tilde{f}=\left(P_{\chi}\left(X, \partial_{X}\right) f\right)^{\sim} \text { on } \operatorname{Exp} \Omega_{1} \text {. }
$$

(iii) The map $P \mapsto \widetilde{P}$ is an order preserving isomorphism of algebras of $\left(\mathbb{D}(\mathfrak{s})^{H}, \times\right)$ onto $\left(\mathbb{D}\left(L_{\chi}\right), \circ\right)$, where the product $\times$ is defined by

$$
(P \times Q)(\xi):=\left.e_{\chi}\left(\partial_{\xi}, \partial_{\eta}\right)(P(\xi) Q(\eta))\right|_{\xi=\eta} .
$$

Remarks. (a) The algebra $\mathbb{D}\left(L_{\chi}\right)$ is therefore commutative if $e_{\chi}(X, Y)=$ $e_{\chi}(Y, X)$ (see Theorem 4.28).

(b) As in 3.3.4 Example 2 we obtain the following corollary: assuming $\mathbb{D}\left(L_{\chi}\right)$ is commutative and $\left(S(\mathfrak{s})^{H}, \cdot\right)$ is a polynomial algebra with generators $P_{1}, \ldots$, $P_{l}$, there exists a unique generalized Duflo isomorphism of algebras $\gamma$ : $\left(S(\mathfrak{s})^{H}, \cdot\right) \rightarrow\left(\mathbb{D}\left(L_{\chi}\right), \circ\right)$ such that $\gamma\left(P_{j}\right)=\widetilde{P_{j}}$ for $j=1, \ldots, l$.

The line bundle $L_{\chi}$ is called special if Definition 3.26 holds with $e_{\chi}=$ 1. Examples are provided by Theorem 4.27. Let us recall the notation $Z_{0}(X, Y)=X+Y$.

Proposition $\mathbf{3 . 2 8}$ Assume $L_{\chi}$ is a special line bundle. Then (i) Let $u, v$ be $H$-invariant distributions on open subsets of $\mathfrak{s}^{\prime}$ and $f \in C^{\infty}(\mathfrak{s})$ such that $\Omega \cap(\operatorname{supp} u \times \operatorname{supp} v) \cap \operatorname{supp}(f \circ Z)$ and $\Omega \cap(\operatorname{supp} u \times \operatorname{supp} v) \cap$ $\operatorname{supp}\left(f \circ Z_{0}\right)$ are compact. Then

$$
\langle\widetilde{u} * \widetilde{v}, \widetilde{f}\rangle=\left\langle\widetilde{u *_{\mathfrak{s}} v}, \widetilde{f}\right\rangle=\left\langle u *_{\mathfrak{s}} v, f\right\rangle .
$$

If $P \in \mathbb{D}(\mathfrak{s})^{H}$ and $u$ is an $H$-invariant distribution on an open subset $U$ of $\mathfrak{s}^{\prime}$

$$
\widetilde{{ }^{t} P u}={ }^{t} \widetilde{P} \widetilde{u},
$$


an equality of distributions on $\operatorname{Exp}\left(\Omega_{1} \cap U\right)$.

(ii) The map $P \mapsto \widetilde{P}$ is an algebra isomorphism of $\mathbb{D}(\mathfrak{s})^{H}$ onto $\mathbb{D}\left(L_{\chi}\right)$.

(iii) Any non-zero differential operator in ${ }^{t} \mathbb{D}(S)$ has an $H$-invariant fundamental solution on $\Omega_{1}$ and is locally solvable.

(iv) Let $\xi \in \mathfrak{s}^{*}$ be such that the coadjoint orbit $H \cdot \xi$ is tempered, and let $T_{\xi}$ be the Fourier transform of an orbital measure on $H \cdot \xi$. Then $\widetilde{T_{\xi}}$ is an $H$-invariant eigendistribution: for all $P \in S(\mathfrak{s})^{H}$

$$
{ }^{t} \widetilde{P} \widetilde{T_{\xi}}=P(i \xi) \widetilde{T_{\xi}} \text { on } \operatorname{Exp} \Omega_{1} .
$$

Proof. (i) to (iii) are proved as in Proposition 3.5.

(iv) The coadjoint action of $H$ on $\mathfrak{s}^{*}$ is defined by $\langle h \cdot \xi, X\rangle=\left\langle\xi, h^{-1} \cdot X\right\rangle$. We assume here that $H \cdot \xi$ carries a measure $\mu_{\xi}$ which is a tempered distribution on $\mathfrak{s}$ such that, for all $h \in H$,

$$
\int_{H \cdot \xi} \varphi(h \cdot \eta) d \mu_{\xi}(\eta)=\left|\operatorname{det}_{\mathfrak{s}} \operatorname{Ad} h\right| \int_{H \cdot \xi} \varphi(\eta) d \mu_{\xi}(\eta) .
$$

The Fourier transform of $\mu_{\xi}$ is $T_{\xi}$ defined by

$$
\left\langle T_{\xi}, f\right\rangle=\int_{H \cdot \xi} \widehat{f} d \mu_{\xi}, \widehat{f}(\eta):=\int_{\mathfrak{s}} f(X) e^{-i\langle\eta, X\rangle} d X
$$

where $d X$ is a Lebesgue measure on $\mathfrak{s}$. Then $T_{\xi}$ is an $H$-invariant distribution on $\mathfrak{s}$,

$$
{ }^{t} P\left(\partial_{X}\right) T_{\xi}=P(i \xi) T_{\xi}
$$

for all $P \in S(\mathfrak{s})^{H}$ and we conclude with (i).

\subsection{Open Problems}

a. Much work remains to be done in order to extend to symmetric spaces and line bundles the Duflo isomorphism for bi-invariant differential operators on Lie groups. As noted in Section 3.3.4, where this is achieved under various assumptions, the problem is to construct an isomorphism of algebras $\varepsilon$ : $\left(S(\mathfrak{s})^{H}, \cdot\right) \rightarrow\left(S(\mathfrak{s})^{H}, \times\right)$, and it seems reasonable to expect that it can be obtained from the $e$-function itself. An example is given by (3.40) for a $G / K$ of the noncompact type, using $e_{\infty}$ - if this limit exists.

As explained in the notes below, a different approach to Duflo's isomorphism for symmetric spaces has been developed by Torossian [52][53] by means of a generalized Harish-Chandra homomorphism; see also his paper [56]. It would be interesting to compare both methods.

One may then look for a further extension of the isomorphism to convolution of $H$-invariant distributions, encompassing the special case of composition of invariant differential operators and possibly leading to solvability theorems for $\mathbb{D}(S)$. See (3.42) and the discussion in Section 3.6.3. 
b. Find a direct proof of (3.33), implying Theorem 3.18, from the general properties of $e(X, Y)$ (Remark (e) in Section 3.6.1).

c. Extend the explicit construction of $e(X, Y)$ in the rank one case (Section 3.7 ) to all spaces $G / K$ of the noncompact type. The existence and properties of $e_{\infty}$ might then be read from the result.

A link was noted in 3.6.3.c between Harish-Chandra's $c$-function and $e_{\infty}$. But can $c(\lambda)$ be explicitly obtained from $e$ ? Some additional information would be needed here about the $K$-invariants on $\mathfrak{p}$ : when $G$ has a complex structure, $e$ and $e_{\infty}$ are identically 1 whereas $c(\lambda)^{-1}=C \prod_{\alpha>0}\langle\alpha, \lambda\rangle$ with some constant factor $C$ (product over all positive roots, see [28] p. 432).

d. Investigate the link between $c(X, Y), e_{\infty}$ and Duistermaat's Jacobian in 3.6.2, so as to clarify the heuristic arguments in this section.

\section{Notes}

When trying to extend to a symmetric space $S=G / H$ the KashiwaraVergne method for Lie groups, the first step is to focus on those spaces for which $H$-invariant convolution products on $S$ and its tangent space $\mathfrak{s}$ exactly correspond under the transfer map . This is the «special» case studied in Section 3.2, taken from [43] and motivated by previous work by Michel Duflo, and by Yves Benoist [10] for nilpotent symmetric spaces.

It soon becomes clear, however, that this property no longer holds for general symmetric spaces (see Proposition 3.7), unless the convolution on $\mathfrak{s}$ is twisted by some factor $e(X, Y)$. The necessity of such an «e-function» appears when computing $\left\langle\widetilde{u} *_{S} \widetilde{v}, \widetilde{f}\right\rangle$ (for $H$ compact and $u, v, f$ functions, say) as an integral over $\mathfrak{s} \times \mathfrak{s}$ by means of the diffeomorphism $\Phi$ of Section 4.2.4; details are given at the beginning of 4.3.2. It was first introduced in [44] and its link with invariant differential operators (Section 3.3) was developed in [45]. Also taken from [45] is the application to spherical functions (Section 3.4), extended here to mean value operators as in [49]. An expansion of mean values similar to Theorem 3.13 was proved by Sigurður Helgason in 1959 (see [29] p. 77) for general Riemannian homogeneous spaces; confining ourselves here to the case of Riemannian symmetric spaces, we obtain a more precise form of his result by means of $e$.

Solvability theorems for invariant differential operators on Riemannian symmetric spaces of the noncompact type have been proved by Helgason $(1964,1973)$, reducing them to operators with constant coefficients by means of the Abel transform; see [29] Chapter V, $\S 1$.

Except for a brief sketch of Proposition 3.21 in [46] (without proof) the material in Sections 3.5 and 3.6 was unpublished. The introduction of $e_{\infty}$ was suggested to me by Mogens Flensted-Jensen. For Riemannian symmetric spaces of the noncompact type, Piotr Graczyk and Patrice Sawyer study in 
[23] an interesting variant of the integral formula (3.28), written as an integral over a certain convex subset of $\mathfrak{a}$.

An obvious prerequisite for constructing a generalized Duflo isomorphism is the commutativity of the algebra $\mathbb{D}(S)$, known (for Riemannian symmetric spaces) from earlier work by Gelfand (1950) and Selberg (1956). In 1964, only assuming the symmetric space $S$ has an invariant volume element, Lichnerowicz [36] proved that the transpose ${ }^{t} D$ of $D \in \mathbb{D}(S)$ is the image of $D$ by the symmetry, whence an easy proof of this commutativity. A purely algebraic proof was later given by Duflo [19] in the more general case of line bundles.

In [52][53] Charles Torossian constructs a generalized Harish-Chandra homomorphism, giving an injective morphism of algebras from the (commutative) algebra of invariant differential operators on the line bundle of halfdensities over $S$ into the field of $H$-invariant fractions of $\mathfrak{s}$. He conjectures that it is actually an isomorphism onto the algebra $S(\mathfrak{s})^{H}$ (the «polynomial conjecture») and proves the conjecture for quadratic or solvable symmetric pairs; in the latter case Torossian's isomorphism is the inverse of our transfer map $\sim$. In [56] he proves a similar result for quadratic symmetric pairs with a skew-invariant bilinear form; see also 4.7.c below.

The first explicit example of an $e$-function was found by Flensted-Jensen for the real hyperbolic space $H^{n}(\mathbb{R})$ (unpublished). Then, drawing inspiration from his calculations with Tom Koornwinder in the more general framework of Jacobi analysis ([22] or [34] §7.1), Flensted-Jensen and I extended the result to all Riemannian symmetric spaces of rank one, leading to Theorem 3.23 (as yet unpublished). The first appearance of the kernel $a$ in (3.46) was in Fritz John's study of iterated spherical means in Euclidean space (1955; see [29] p. 356). The kernel $b$ in (3.47) was introduced by Flensted-Jensen and Koornwinder in [22] $\S 4$.

An explicit $e$-function for Riemannian symmetric spaces of the compact type is put forward in Anthony Dooley's survey paper [17]; the detailed proof hasn't appeared yet.

Taken from [47], the extension to line bundles in Section 3.8 answers a question asked me by Duflo. 


\section{Chapter 4}

\section{$e$-Functions and the Campbell-Hausdorff Formula}

Throughout this chapter $S=G / H$ denotes a connected and simply connected symmetric space, where $G$ is a simply connected (we may assume this, see 0.4) real Lie group with involutive automorphism $\sigma$ and $H$ is the connected component of the identity in the fixed point subgroup of $\sigma$ in $G$. The main use of these topological assumptions is to specify the open sets we are working on. We still denote by $\sigma$ the corresponding automorphism of the Lie algebra $\mathfrak{g}$, whence the decomposition $\mathfrak{g}=\mathfrak{h} \oplus \mathfrak{s}$.

The goal of this chapter is to extend to any such space $S$ the KashiwaraVergne method of Chapter 1 for Lie groups. We show that $S$ admits an $e$-function, which can be constructed by means of the Campbell-Hausdorff formula, and we prove some properties of this function. As explained in Section 3.1.2 we want a function $e(X, Y)$ on an open subset of $\mathfrak{s} \times \mathfrak{s}$, relating convolutions of invariant distributions on $S$ and $\mathfrak{s}$, namely

$\left\langle u(X) \otimes v(Y), \frac{j(X) j(Y)}{j(Z(X, Y))} f(Z(X, Y))\right\rangle=\langle u(X) \otimes v(Y), e(X, Y) f(X+Y)\rangle$

for any $H$-invariant distributions $u, v$ and any function $f$ (with suitable supports), where $Z(X, Y) \in \mathfrak{s}$ is defined by

$$
\operatorname{Exp} Z(X, Y)=\exp X \cdot \operatorname{Exp} Y .
$$

This will be done by

- studying $Z(X, Y)$, defined on a domain $\mathcal{O}$ in $\mathfrak{s} \times \mathfrak{s}$ (Section 4.2.2), in order to obtain a symmetric space analog of the Campbell-Hausdorff formula, written by means of Lie series $A, B, C$ in a specific form similar 
to the first Kashiwara-Vergne equation (KV1) for Lie groups (Section $4.2 .3)$

- then transforming $Z(X, Y)$ into its flat analog $X+Y$ by a local diffeomorphism $\Phi$ of $\mathfrak{s} \times \mathfrak{s}$ (Section 4.2.4), linked to contractions of the symmetric space into its tangent space at the origin (Section 4.1) and given by adjoint action of elements $a(X, Y), b(X, Y)$ of $H$, viz. $Z(\Phi(X, Y))=$ $X+Y$ with $\Phi(X, Y)=(a(X, Y) \cdot X, b(X, Y) \cdot Y)$. This $\Phi$ is the symmetric space analog of $\mathbf{F}$ in Section 1.3 for Lie groups.

Along the way we point out some properties of the element $c(X, Y)=$ $a(X, Y)^{-1} b(X, Y)$ of $H$ and of the $H$-component $h(X, Y)$ defined by $e^{Z(X, Y)} h(X, Y)=e^{X} e^{Y}$ (Section 4.2.5). The latter is needed when dealing with line bundles.

The Jacobian of $\Phi$ is an essential factor of our $e$-function, constructed in Section 4.3.2 and studied in detail in 4.4. Roughly speaking, $e$ conveys the lack of validity of the second Kashiwara-Vergne equation (KV2) for general symmetric spaces; see Proposition 4.19 for a precise statement.

The results are extended in Section 4.5 to the line bundle over $S$ defined by a character $\chi$ of $H$. The Taylor expansions in Section 4.6 aim at getting a better grasp of the objects introduced in this chapter. Section 4.7 suggests a few open problems.

Though it draws inspiration from the Kashiwara-Vergne method for Lie groups the present chapter can be read, to a large extent, independently from Chapter 1. The main results are Theorem 4.6 (construction of $\Phi$ ), Theorem 4.12 (construction of a «Campbell-Hausdorff $e$-function»), Theorem 4.20 (structure of this $e$-function), Theorem 4.22 ( $e=1$ identically for solvable symmetric spaces and strongly symmetric spaces), Theorem 4.24 (symmetry $e(X, Y)=e(Y, X)$ in many cases, implying the commutativity of the algebra of invariant differential operators on $S$ ) and their extensions to line bundles (Theorems 4.25, 4.27, 4.28). Theorem 4.16 expresses invariant differential operators on $S$ in exponential coordinates by means of the $e$-function.

\subsection{Contractions of Symmetric Spaces}

Though not essential for the sequel this section explains the geometrical background of all subsequent work in this chapter with a parameter $t$.

For the general theory of symmetric spaces we refer to the classical books by Kobayashi-Nomizu [31], Loos [37] and, for the Riemannian case, Helgason [27]. Let us simply recall the equivalence between the category of simply connected pointed symmetric spaces $(S, o)$ and the category of finite dimensional Lie triple systems $(\mathfrak{s},[., .,]$.$) . Here \mathfrak{s}$ is the tangent space to $S$ at the origin $o$, with trilinear structure

$$
[X, Y, Z]:=-R_{o}(X, Y) Z=[[X, Y], Z]
$$


where $R_{o}$ is the curvature tensor at $o$ and the latter brackets are the Lie brackets of $\mathfrak{g}$; see [37] chapter II for details.

Given a Lie triple system $(\mathfrak{s},[., .,]$.$) and a real parameter t$, we define the contracted Lie triple system $\mathfrak{s}_{t}$ as the vector space $\mathfrak{s}$ with trilinear product

$$
[X, Y, Z]_{t}:=t^{2}[X, Y, Z] .
$$

Let $\left(S_{t}, o\right)$ be the corresponding simply connected pointed symmetric space, unique up to isomorphism; its curvature tensor at the origin is $t^{2} R_{o}$. The subscript $t$ will always refer to notions relative to this contracted space.

For $t \neq 0$ the map $f^{t}: X \mapsto t X$ is an isomorphism of the Lie triple system $\mathfrak{s}_{t}$ onto $\mathfrak{s}$. Still denoting by $f^{t}$ the corresponding isomorphism of $S_{t}$ onto $S=S_{1}$ we have

$$
f^{t}\left(\operatorname{Exp}_{t} X\right)=\operatorname{Exp} t X \text { for } X \in \mathfrak{s}, t \neq 0 .
$$

The flat space $S_{0}$ is not, in general, isomorphic with other $S_{t}$ 's; it can be identified with the tangent vector space at the origin of $S$. We call this process contraction of $S$ into its tangent space.

If $\mathfrak{s}$ is given by a symmetric Lie algebra $(\mathfrak{g}, \sigma)$, we may extend $f^{t}$ to $\mathfrak{g}=\mathfrak{h} \oplus \mathfrak{s}$ by $f^{t}(A+X)=A+t X, A \in \mathfrak{h}, X \in \mathfrak{s}$. For $t \neq 0, f^{t}$ is an isomorphism of $\mathfrak{g}_{t}$ onto $\mathfrak{g}=\mathfrak{g}_{1}$ where $\mathfrak{g}_{t}$ is the vector space $\mathfrak{g}$ with Lie bracket

$$
[A+X, B+Y]_{t}:=\left([A, B]+t^{2}[X, Y]\right)+([A, Y]-[B, X]),
$$

$A, B \in \mathfrak{h}, X, Y \in \mathfrak{s}$, and $\mathfrak{s}_{t}$ is given by the symmetric Lie algebra $\left(\mathfrak{g}_{t}, \sigma\right)$. This definition agrees with the classical «contraction of $\mathfrak{g}$ with respect to $\mathfrak{h}$ 》 (Dooley-Rice [16]), or with the contraction of a filtered Lie algebra into its graded algebra (Guillemin-Sternberg [25] p. 447). Besides $\mathfrak{g}_{0}$ is the semidirect product of the vector space $\mathfrak{s}$ (an abelian Lie algebra) by $\mathfrak{h}$.

Likewise, when $S$ is given by $(G, H, \sigma)$ the space $S_{0}$ is $G_{0} / H$ where $G_{0}$ is the semi-direct product $\mathfrak{s} \times H$.

The dual $\mathfrak{s}_{*}$ of a Lie triple system $\mathfrak{s}$ is defined as the same vector space $\mathfrak{s}$ with trilinear product $[X, Y, Z]_{*}=-[X, Y, Z]([37]$ p. 150, [31] p. 253); this gives, in particular, the classical duality between compact and noncompact types. An obvious but useful remark is that $\mathfrak{s}_{*}$ may be formally considered as $\mathfrak{s}_{t}$ with $t=i$.

Example. As a typical example let us consider the hyperbolic unit disk $S=H^{2}(\mathbb{R})$ with $G=S U(1,1), H=S O(2)$. Then $S_{t}$ can be realized, for $t>0$, as the disk $|z|<1 / t$ in $\mathbb{C}$ with Riemannian metric

$$
d s^{2}=\frac{4|d z|^{2}}{\left(1-t^{2}|z|^{2}\right)^{2}} .
$$

Here $f^{t}(z)=t z$ and the curvature of $S_{t}$ is $-t^{2}$. The space $S_{0}$ is the Euclidean plane and $G_{0}$ its motion group. The same $S_{0}$ and $G_{0}$ arise from the unit sphere with $G=S O(3), H=S O(2)$ too, with $S_{t}$ realized then as a sphere with radius $1 / t$ as a Riemannian submanifold of $\mathbb{R}^{3}$. 


\subsection{The Diffeomorphism $\Phi$}

\subsubsection{Lie Series}

We work with Lie series, that is series of Lie brackets in $\mathfrak{g}$ of the form

$$
A(X, Y)=a X+b Y+c[X, Y]+d[X,[X, Y]]+e[Y,[X, Y]]+\cdots
$$

(where $a, b$, etc. are scalars). In this chapter Lie series are assumed to converge for $(X, Y)$ in some neighborhood of the origin of $\mathfrak{g} \times \mathfrak{g}$. More precisely, writing (in multi-index notation)

$$
A(X, Y)=a X+\sum_{\alpha, \beta} a_{\alpha \beta} Z_{\alpha \beta} \text { with } Z_{\alpha \beta}=x^{\alpha_{1}} y^{\beta_{1}} x^{\alpha_{2}} y^{\beta_{2}} \cdots y^{\beta_{n-1}} x^{\alpha_{n}} Y,
$$

$x=\operatorname{ad} X, y=\operatorname{ad} Y$, we assume $\sum_{\alpha, \beta}\left|a_{\alpha \beta}\right| R^{|\alpha|+|\beta|}<\infty$ for some $R>0$. If we pick a norm on $\mathfrak{g}$ such that $\|[X, Y]\| \leq\|X\|\|Y\|$, the above series is absolutely convergent for $\|X\|<R$ and $\|Y\|<R$.

A Lie series is equivariant under any automorphism of the Lie algebra, in particular $g \cdot A(X, Y)=A(g \cdot X, g \cdot Y)$ for $g \in G$ (adjoint action of the group) and $\sigma A(X, Y)=A(\sigma X, \sigma Y)$. An even Lie series maps $\mathfrak{s} \times \mathfrak{s}$ into $\mathfrak{h}$.

If $A$ is a Lie series then

$$
\operatorname{ad} A(X, Y)=\sum_{\alpha, \beta} a_{\alpha \beta}^{\prime} u_{\alpha \beta} \text { with } u_{\alpha \beta}=x^{\alpha_{1}} y^{\beta_{1}} x^{\alpha_{2}} y^{\beta_{2}} \cdots x^{\alpha_{n}} y^{\beta_{n}},
$$

a (non-commutative) power series in $x, y$. This follows from the identity (where the $C_{p}^{r}$ 's are the binomial coefficients and $V \in \mathfrak{g}$ is arbitrary)

$$
\operatorname{ad}\left(x^{p} y^{q} V\right)=\sum_{r, s}(-1)^{r+s} C_{p}^{r} C_{q}^{s} x^{p-r} y^{q-s}(\operatorname{ad} V) y^{s} x^{r}
$$

applied to each $Z_{\alpha \beta}$. Since $\left\|\operatorname{ad} Z_{\alpha \beta}\right\| \leq\left\|Z_{\alpha \beta}\right\| \leq R^{|\alpha|+|\beta|+1}$ (with the operator norm for endomorphisms of $\mathfrak{g}$ ) we have

$$
\left\|\sum_{|\alpha|=k,|\beta|=l} a_{\alpha \beta}^{\prime} u_{\alpha \beta}\right\|=\left\|\sum_{|\alpha|=k,|\beta|=l-1} a_{\alpha \beta} \operatorname{ad} Z_{\alpha \beta}\right\| \leq \sum_{|\alpha|=k,|\beta|=l-1}\left|a_{\alpha \beta}\right| R^{k+l}
$$

and the series (4.1) converges in the following sense:

$$
\sum_{k, l}\left\|\sum_{|\alpha|=k,|\beta|=l} a_{\alpha \beta}^{\prime} u_{\alpha \beta}\right\|<\infty \text { for }\|X\|<R,\|Y\|<R .
$$

The partial differentials $\partial_{X} A, \partial_{Y} A$ defined by $\partial_{X} A(X, Y) V=\left.\partial_{t} A(X+t V, Y)\right|_{t=0}, \partial_{Y} A(X, Y) V=\left.\partial_{t} A(X, Y+t V)\right|_{t=0}$ 
for $V \in \mathfrak{g}$ are also given by series of the form (4.1). This follows from the identity, for $p \geq 1$,

$$
\partial_{X}\left(x^{p} y^{q} F(X, Y)\right)=x^{p} y^{q} \partial_{X} F(X, Y)-\sum_{0 \leq r<p} x^{p-r-1} \operatorname{ad}\left(x^{r} y^{q} F(X, Y)\right),
$$

applied to each $Z_{\alpha \beta}$.

A linear endomorphism $u: \mathfrak{g} \rightarrow \mathfrak{g}$ commutes with $\sigma$ if and only if it maps $\mathfrak{h}$ into $\mathfrak{h}$ and $\mathfrak{s}$ into $\mathfrak{s}$. Thus

$$
u \sigma=\sigma u \text { implies } \operatorname{tr}_{\mathfrak{g}} u=\operatorname{tr}_{\mathfrak{h}} u+\operatorname{tr}_{\mathfrak{s}} u
$$

where $\operatorname{tr}_{\mathfrak{h}} u$ etc. means the trace of the restriction of $u$ to $\mathfrak{h}$ etc.

An endomorphism $u$ of $\mathfrak{g}$ anticommutes with $\sigma$ if and only if it maps $\mathfrak{h}$ into $\mathfrak{s}$ and $\mathfrak{s}$ into $\mathfrak{h}$. Then, $u, v$ being two endomorphisms of $\mathfrak{g}$,

$$
u \sigma=-\sigma u \text { and } v \sigma=-\sigma v \text { imply } \operatorname{tr}_{\mathfrak{h}} u v=\operatorname{tr}_{\mathfrak{s}} v u .
$$

If furthermore $u v=v u$ then (4.2) also applies to $u v$ and $\operatorname{tr}_{\mathfrak{g}} u v=2 \operatorname{tr}_{\mathfrak{h}} u v=$ $2 \operatorname{tr}_{\mathfrak{s}} u v$.

These trace identities will be used several times with endomorphisms defined by series similar to (4.1): if $X, Y \in \mathfrak{s}$ we have $\sigma x=-x \sigma, \sigma y=-y \sigma$ and $u$ commutes (resp. anticommutes) with $\sigma$ if it is given by a series of even (resp. odd) terms.

\subsection{2 $\mathcal{O}, Z, h$}

We need to gather some information on our basic tool, the map $Z: \mathcal{O} \rightarrow \mathfrak{s}$ defined on an open set $\mathcal{O} \subset \mathfrak{s} \times \mathfrak{s}$ by

$$
\operatorname{Exp} Z(X, Y)=\exp X \cdot \operatorname{Exp} Y .
$$

We first discuss its domain $\mathcal{O}$ then $Z$ and, at the same time, the $H$-component $h(X, Y)$ defined by $e^{Z(X, Y)} h(X, Y)=e^{X} e^{Y}$, which is useful when dealing with line bundles.

All maps we consider will be defined on suitable neighborhoods of the origin only. As in 1.3 let $\mathfrak{g}^{\prime}$ be a connected open neighborhood of 0 in $\mathfrak{g}$, invariant under all automorphisms of $\mathfrak{g}$ and all maps $X \mapsto t X, t \in[-1,1]$, and such that $\exp : \mathfrak{g}^{\prime} \rightarrow \exp \mathfrak{g}^{\prime}$ is a diffeomorphism; $\log$ will denote the inverse map. The group $G$ being simply connected we may take as $\mathfrak{g}^{\prime}$ the set of all $X \in \mathfrak{g}$ such that $|\operatorname{Im} \lambda|<\pi$ for all eigenvalues $\lambda$ of $\operatorname{ad} X$ (see [58] p. 113). Having chosen such a $\mathfrak{g}^{\prime}$ let

$$
\mathcal{U}:=\left\{(X, Y) \mid 2 X, 2 Y \in \mathfrak{g}^{\prime} \text { and } e^{2 t X} e^{2 t Y} \in \exp \mathfrak{g}^{\prime} \text { for all } t \in[0,1]\right\} .
$$

Slightly different from (1.10) in use throughout Chapter 1, this definition of $\mathcal{U}$ with factors 2 is more convenient when working with symmetric spaces. By 
Lemma 1.1 the set $\mathcal{U}$ is a connected open neighborhood of $(0,0)$ in $\mathfrak{g} \times \mathfrak{g}$, invariant under the maps $(X, Y) \mapsto(t X, t Y), t \in[-1,1],(X, Y) \mapsto(Y, X)$ and $(X, Y) \mapsto(A X, A Y)$ with $A \in \operatorname{Ad} G$ or $A=\sigma$. Thus $(X, Y) \mapsto \frac{1}{2} \log e^{2 X} e^{2 Y}$ defines an analytic map $\mathcal{U} \rightarrow \frac{1}{2} \mathfrak{g}^{\prime}$.

For the symmetric space $S=G / H$ the set $\mathfrak{s}^{\prime}=\mathfrak{s} \cap \frac{1}{2} \mathfrak{g}^{\prime}$ of Lemma 3.1 is a connected open neighborhood of 0 in $\mathfrak{s}$, invariant under $\operatorname{Ad}_{G} H$ and all maps $X \mapsto t X, t \in[-1,1]$, such that Exp $: \mathfrak{s}^{\prime} \rightarrow \operatorname{Exp} \mathfrak{s}^{\prime}$ is a diffeomorphism. Let

$$
\mathcal{O}:=\left\{(X, Y) \mid X, Y \in \mathfrak{s}^{\prime} \text { and } \exp t X \cdot \operatorname{Exp} t Y \in \operatorname{Exp} \mathfrak{s}^{\prime} \text { for all } t \in[0,1]\right\} .
$$

This $\mathcal{O}$ is a natural domain for studying the map $(X, Y) \mapsto \exp X \cdot \operatorname{Exp} Y$. In geometrical terms, denoting by $s_{x}$ the symmetry with respect to the point $x$ in $S$,

$\operatorname{Exp} Z(X, Y)=\exp X \cdot \operatorname{Exp} Y=s_{\operatorname{Exp}(X / 2)} s_{o}(\operatorname{Exp} Y)=s_{\operatorname{Exp}(X / 2)}(\operatorname{Exp}(-Y))$.

Remark. If $G$ is a solvable exponential group we may take $\mathfrak{g}^{\prime}=\mathfrak{g}$, whence $\exp \mathfrak{g}^{\prime}=G, \mathcal{U}=\mathfrak{g} \times \mathfrak{g}, \mathfrak{s}^{\prime}=\mathfrak{s}$ and $\mathcal{O}=\mathfrak{s} \times \mathfrak{s}$. If Exp is a global diffeomorphism of $\mathfrak{s}$ onto $S$ (e.g. if $S$ is a Riemannian symmetric space of the noncompact type, see [27] p. 253) we may take $\mathfrak{s}^{\prime}=\mathfrak{s}, \mathcal{O}=\mathfrak{s} \times \mathfrak{s}$, even though $\mathfrak{g}^{\prime}$ is smaller than $\mathfrak{g}$ in that case.

Lemma 4.1 We have $\mathcal{O}=\mathcal{U} \cap\left(\mathfrak{s}^{\prime} \times \mathfrak{s}^{\prime}\right)=\mathcal{U} \cap(\mathfrak{s} \times \mathfrak{s})$, and $\mathcal{O}$ is a connected open neighborhood of $(0,0)$ in $\mathfrak{s} \times \mathfrak{s}$, invariant under all maps

(i) $(X, Y) \mapsto(t X, t Y)$ for $t \in[-1,1]$

(ii) $(X, Y) \mapsto(h \cdot X, h \cdot Y)$ for $h \in H$

(iii) $(X, Y) \mapsto(Y, X)$

Proof. The equality $\mathcal{U} \cap\left(\mathfrak{s}^{\prime} \times \mathfrak{s}^{\prime}\right)=\mathcal{U} \cap(\mathfrak{s} \times \mathfrak{s})$ is clear from the definitions of $\mathcal{U}$ and $\mathfrak{s}^{\prime}$. Let $(X, Y) \in \mathfrak{s}^{\prime} \times \mathfrak{s}^{\prime}$. To prove that $(X, Y)$ belongs to $\mathcal{O}$ if and only if it belongs to $\mathcal{U}$, let $g:=e^{t X} e^{t Y}, t \in[0,1]$. Using the symmetry $\sigma$ we have $\sigma X=-X, \sigma Y=-Y$ therefore

$$
e^{2 t X} e^{2 t Y}=e^{t X} g(\sigma g)^{-1} e^{-t X} .
$$

Due to the Ad $G$-invariance of $\mathfrak{g}^{\prime}$ we see that $e^{2 t X} e^{2 t Y} \in \exp \mathfrak{g}^{\prime}$ is equivalent to $g(\sigma g)^{-1} \in \exp \mathfrak{g}^{\prime}$, that is $g(\sigma g)^{-1}=e^{2 Z}$ with $Z \in \frac{1}{2} \mathfrak{g}^{\prime}$, and in fact $Z \in$ $\mathfrak{s}^{\prime}$ (as follows from the behavior under $\sigma$ and the injectivity of exp on $\mathfrak{g}^{\prime}$ ). Therefore $e^{2 t X} e^{2 t Y}$ belongs to exp $\mathfrak{g}^{\prime}$ if and only if there exists $Z \in \mathfrak{s}^{\prime}$ such that $e^{-Z} g=\sigma\left(e^{-Z} g\right)$ or else

$$
g=e^{t X} e^{t Y}=e^{Z} h
$$

where $h$ belongs to the fixed point subgroup $G^{\sigma}$ of $\sigma$ in $G$. But $g, Z$ and $h$ are continuous functions of $t \in[0,1]$; for $t=0$ we have $g H=\operatorname{Exp} 0=\operatorname{Exp} Z$ hence $Z=0$ by the injectivity of Exp on $\mathfrak{s}^{\prime}$, and $h=e$. It follows that, for all $t, h$ belongs to the identity component of $G^{\sigma}$, which equals $H$ by our 
assumptions. We conclude that $e^{2 t X} e^{2 t Y}$ belongs to $\exp \mathfrak{g}^{\prime}$ for all $t \in[0,1]$ if and only if $g=e^{Z} h$ with $Z \in \mathfrak{s}^{\prime}$ and $h \in H$, that is $\exp t X \cdot \operatorname{Exp} t Y \in \operatorname{Exp} \mathfrak{s}^{\prime}$ for all $t \in[0,1]$. This implies our claim.

The invariance properties of $\mathcal{O}$ are immediate consequences of the corresponding properties of $\mathcal{U}$.

For $(X, Y) \in \mathcal{O}$ the point $\exp X \cdot \operatorname{Exp} Y=e^{X} e^{Y} H$ lies in $S^{\prime}=\operatorname{Exp} \mathfrak{s}^{\prime}$, whence a unique $Z \in \mathfrak{s}^{\prime}$ such that $\operatorname{Exp} Z=\exp X \cdot \operatorname{Exp} Y$. This defines a map $Z: \mathcal{O} \rightarrow \mathfrak{s}^{\prime}$, a symmetric space analogue of the Campbell-Hausdorff function, expressing the action of $G$ on $S=G / H$ in exponential coordinates. The following proposition extends $Z$ to $\mathcal{U}$, as well as the corresponding $H$ component. We write $x=\operatorname{ad} X, y=\operatorname{ad} Y, z=\operatorname{ad} Z(X, Y)$.

Proposition 4.2 The formulas

$$
\begin{aligned}
Z(X, Y) & :=\frac{1}{2} e^{-x} \log \left(e^{2 X} e^{2 Y}\right)=\frac{1}{2} e^{x} \log \left(e^{2 Y} e^{2 X}\right)=\frac{1}{2} \log \left(e^{X} e^{2 Y} e^{X}\right) \\
h(X, Y) & :=e^{-Z(X, Y)} e^{X} e^{Y}
\end{aligned}
$$

define an analytic map $Z: \mathcal{U} \rightarrow \frac{1}{2} \mathfrak{g}^{\prime}$, given by an odd Lie series, and an analytic map $h: \mathcal{U} \rightarrow G$ such that, for all $(X, Y) \in \mathcal{U}$,

(i) $Z(-X,-Y)=-Z(X, Y)$ and $h(-X,-Y)=h(X, Y)$

(ii) $Z(g \cdot X, g \cdot Y)=g \cdot Z(X, Y)$ and $h(g \cdot X, g \cdot Y)=g h(X, Y) g^{-1}$ for all $g \in G$

(iii) $Z(Y, X)=h(X, Y)^{-1} \cdot Z(X, Y)$ and $h(Y, X)=h(X, Y)^{-1}$

(iv) $\psi(h(X, Y))=1$ for any character $\psi$ of the group $G$.

(v) For $(X, Y) \in \mathcal{U}, Z=Z(X, Y)$ and $h=h(X, Y)$ we have

$$
\frac{\operatorname{sh} z}{z} \partial_{X} Z=\operatorname{Ad} h \circ\left(\operatorname{ch} y \frac{\operatorname{sh} x}{x}+\operatorname{sh} y \frac{\operatorname{ch} x-1}{x}\right), \frac{\operatorname{sh} z}{z} \partial_{Y} Z=\operatorname{Ad} h \circ \frac{\operatorname{sh} y}{y},
$$

as endomorphisms of $\mathfrak{g}$.

(vi) When restricted to $(X, Y) \in \mathcal{O}, Z(X, Y)$ and $h(X, Y)$ are characterized by

$$
Z(X, Y) \in \mathfrak{s}^{\prime}, h(X, Y) \in H \text { and } e^{X} e^{Y}=e^{Z(X, Y)} h(X, Y) .
$$

Proof. For $(X, Y) \in \mathcal{U}$ the point $e^{-X}\left(e^{2 X} e^{2 Y}\right) e^{X}=e^{X}\left(e^{2 Y} e^{2 X}\right) e^{-X}=$ $e^{X} e^{2 Y} e^{X}$ belongs to $\exp \mathfrak{g}^{\prime}$, by the $\operatorname{Ad} G$-invariance of $\mathfrak{g}^{\prime}$. Thus $Z(X, Y)$ is well-defined and belongs to $\frac{1}{2} \mathfrak{g}^{\prime}$.

(i) First $Z(-X,-Y)=\frac{1}{2} \log \left(e^{-X} e^{-2 Y} e^{-2 X}\right)=-Z(X, Y)$. Then

$$
h(-X,-Y)=e^{Z(X, Y)} e^{-X} e^{-Y}=e^{-Z(X, Y)} e^{X} e^{Y}=h(X, Y)
$$

because $e^{2 Z(X, Y)}=e^{X} e^{2 Y} e^{X}$.

(ii) is straightforward.

(iii) The formula for $Z(Y, X)$ follows from

$$
Z(Y, X)=\frac{1}{2} e^{-y} \log \left(e^{2 Y} e^{2 X}\right)=e^{-y} e^{-x} Z(X, Y)
$$


and $h(X, Y)^{-1}=e^{-Y} e^{-X} e^{Z(X, Y)}$. Therefore, remembering (4.4),

$h(Y, X)=e^{-Z(Y, X)} e^{Y} e^{X}=h(X, Y)^{-1} e^{-Z(X, Y)} h(X, Y) e^{Y} e^{X}=h(X, Y)^{-1}$.

(iv) Similarly

$$
h(X, Y)^{2}=\left(e^{Z(X, Y)} e^{-X} e^{-Y}\right)\left(e^{-Z(X, Y)} e^{X} e^{Y}\right)
$$

thus $\psi(h(X, Y))^{2}=1$, whence $\psi(h(X, Y))=1$ since $h(0,0)=e$ and $\mathcal{U}$ is connected.

(v) Let $L_{g}$, resp. $R_{g}$, denote the left, rep. right, translation by $g$ in $G$. Using $e^{2 Z}=e^{X} e^{2 Y} e^{X}$ and the differential of exp at $X$ :

$$
D_{X} \exp =D_{e} L_{e^{X}} \circ \frac{1-e^{-x}}{x}
$$

differentiation with respect to $X$ gives

$$
D_{e} L_{e^{2 Z}} \frac{1-e^{-2 z}}{z} \partial_{X} Z=\left(D_{e^{X}} L_{e^{X} e^{2 Y}}+D_{e^{X}} R_{e^{2 Y} e^{X}}\right) \circ D_{X} \exp .
$$

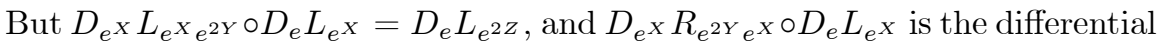
at $e$ of the map

$$
g \mapsto e^{X} g e^{2 Y} e^{X}=e^{X} g e^{-X} e^{2 Z}=e^{2 Z}\left(e^{-2 Z} e^{X}\right) g\left(e^{-2 Z} e^{X}\right)^{-1},
$$

thus

$$
D_{e^{X}} R_{e^{2 Y} e^{X}} \circ D_{e} L_{e^{X}}=D_{e} L_{e^{2 Z}} \circ \operatorname{Ad}\left(e^{-2 Z} e^{X}\right)=D_{e} L_{e^{2 Z}} \circ e^{-2 z} e^{x} .
$$

Simplifying by $D_{e} L_{e^{2 Z}}$ and multiplying by $e^{z}$ on the left we obtain

$$
2 \frac{\operatorname{sh} z}{z} \partial_{X} Z=e^{z} \frac{1-e^{-x}}{x}+e^{-z} \frac{e^{x}-1}{x} .
$$

From (4.4) we know that $e^{X} e^{Y}=e^{Z} h$ and $e^{-X} e^{-Y}=e^{-Z} h$ therefore, by the adjoint representation,

$$
e^{z}=\operatorname{Ad} h \circ e^{y} e^{x}, e^{-z}=\operatorname{Ad} h \circ e^{-y} e^{-x}
$$

whence

$$
2 \frac{\operatorname{sh} z}{z} \partial_{X} Z=\operatorname{Ad} h \circ\left(e^{y} \frac{e^{x}-1}{x}+e^{-y} \frac{1-e^{-x}}{x}\right) .
$$

Writing $e^{x}-1=\operatorname{ch} x-1+\operatorname{sh} x$ and $1-e^{-x}=1-\operatorname{ch} x+\operatorname{sh} x$ we obtain the first result.

Computing $\partial_{Y} Z$ is similar but easier, and left to the Reader.

(vi) Restricting now to $X, Y \in \mathfrak{s}^{\prime}$, that is $(X, Y) \in \mathcal{O}$, we obtain by means of the symmetry $\sigma$

$$
\sigma Z(X, Y)=\frac{1}{2} \log \left(e^{-X} e^{-2 Y} e^{-X}\right)=-Z(X, Y)
$$


therefore $Z(X, Y)$ belongs to $\mathfrak{s} \cap \frac{1}{2} \mathfrak{g}^{\prime}=\mathfrak{s}^{\prime}$. Then, by (4.4),

$$
\sigma h(X, Y)=e^{Z(X, Y)} e^{-X} e^{-Y}=h(X, Y) .
$$

Thus $h(X, Y)$ belongs to the fixed point subgroup of $\sigma$ in $G$, in fact to its identity component since $h(\mathcal{O})$ is connected, therefore to $H$. Conversely, given $(X, Y) \in \mathcal{O}, e^{X} \cdot \operatorname{Exp} Y$ belongs to $\operatorname{Exp} \mathfrak{s}^{\prime}$ whence a unique $Z \in \mathfrak{s}^{\prime}$ such that $e^{X} \cdot \operatorname{Exp} Y=\operatorname{Exp} Z$, then a unique $h \in H$ such that $e^{X} e^{Y}=e^{Z} h$.

\subsection{3 $A, B, C$}

We can now extend to symmetric spaces the Kashiwara-Vergne method of Chapter 1. The present section is motivated by our aim, Theorem 4.6 below: construct a diffeomorphism $\Phi$ of $\mathfrak{s} \times \mathfrak{s}$ (near the origin) transforming the map $Z$ of 4.2.2 into its flat analogue i.e. $Z \circ \Phi(X, Y)=X+Y$. Following Moser's method we shall take $\Phi=\Phi_{1}$ where $\Phi_{t}:(X, Y) \mapsto\left(X_{t}, Y_{t}\right), 0 \leq t \leq 1$, is a one-parameter deformation of the identity $\Phi_{0}$ given by the flow of a suitably chosen (time-dependent) vector field on $\mathfrak{s} \times \mathfrak{s}$. More precisely we want $\Phi_{t}$ to be given by the adjoint action of elements $a_{t}, b_{t}$ of $H$ (depending on $X$ and $Y)$ :

$$
\begin{aligned}
\Phi_{t}(X, Y)= & \left(X_{t}, Y_{t}\right)=\left(a_{t}(X, Y) \cdot X, b_{t}(X, Y) \cdot Y\right) \\
& \text { and } Z_{t}\left(X_{t}, Y_{t}\right)=X+Y
\end{aligned}
$$

with $Z_{t}(X, Y):=t^{-1} Z(t X, t Y)$ for $t>0$, which extends by $Z_{0}(X, Y)=$ $X+Y$. The latter line is thus equivalent to

$$
\partial_{t} Z_{t}+\left(\partial_{X} Z_{t}\right) \partial_{t} X_{t}+\left(\partial_{Y} Z_{t}\right) \partial_{t} Y_{t}=0
$$

where all derivatives of $Z_{t}$ are taken at $\left(X_{t}, Y_{t}\right)$. In a matrix Lie group we have $X_{t}=a_{t} \cdot X=a_{t} X a_{t}^{-1}$ whence $\partial_{t} X_{t}=\left[\left(\partial_{t} a_{t}\right) a_{t}^{-1}, X_{t}\right]$. This extends to the general case as

$$
\begin{gathered}
\partial_{t} X_{t}=\left[A_{t}\left(X_{t}, Y_{t}\right), X_{t}\right], \partial_{t} Y_{t}=\left[B_{t}\left(X_{t}, Y_{t}\right), Y_{t}\right] \\
A_{t}\left(X_{t}, Y_{t}\right)=\left(D_{e} R_{a_{t}}\right)^{-1} \partial_{t} a_{t}, B_{t}\left(X_{t}, Y_{t}\right)=\left(D_{e} R_{b_{t}}\right)^{-1} \partial_{t} b_{t},
\end{gathered}
$$

where $R_{a}$ denotes the right translation by $a$ in $H$ and $D_{e} R_{a}$ its tangent map at the identity. Thus $A_{t}$ and $B_{t}$ belong to $\mathfrak{h}$ and depend on $(X, Y)$.

Summarizing, we want a map $(t, X, Y) \mapsto\left(A_{t}(X, Y), B_{t}(X, Y)\right)$ from a neighborhood of the origin in $\mathbb{R} \times \mathfrak{s} \times \mathfrak{s}$ into $\mathfrak{h} \times \mathfrak{h}$ such that

$$
\partial_{t} Z_{t}=\left(\partial_{X} Z_{t}\right)\left[X, A_{t}\right]+\left(\partial_{Y} Z_{t}\right)\left[Y, B_{t}\right]
$$

an equality now written at $(X, Y)$ instead of $\left(X_{t}, Y_{t}\right)$ above. The construction of $A_{t}$ and $B_{t}$ will be given by Proposition 4.5 and the properties of $a_{t}, b_{t}$ and $\Phi_{t}$ by Theorem 4.6. We first give equivalent forms of this equation. 
Proposition 4.3 Let $Z_{t}(X, Y):=t^{-1} Z(t X, t Y)$, let $A, B$ be given elements of $\mathfrak{g}$ and $C=B-A$. For $(X, Y) \in \mathcal{U}$ the following relations are equivalent (with $x=\operatorname{ad} X, y=\operatorname{ad} Y)$ :

(i) $\left.\partial_{t} Z_{t}(X, Y)\right|_{t=1}=\left(\partial_{X} Z\right)(X, Y)[X, A]+\left(\partial_{Y} Z\right)(X, Y)[Y, B]$

(i') $\left.\partial_{t} Z_{t}(X, Y)\right|_{t=1}=[Z(X, Y), A]+\left(\partial_{Y} Z\right)(X, Y)[Y, C]$

(ii) $Z(Y, X)=(\operatorname{ch} y)(X+Y)-(\operatorname{ch} y \operatorname{sh} x+\operatorname{sh} y \operatorname{ch} x) A-(\operatorname{sh} y) C$.

Later on (Proposition 4.5) $A$ and $C$ will be chosen, depending on $(X, Y)$, so that (ii) holds identically.

Proof. (i) $\Leftrightarrow\left(i^{\prime}\right)$ is immediate from (a) in the next lemma.

Lemma 4.4 (a) Let $\mathcal{U}$ be an open subset of $\mathfrak{g} \times \mathfrak{g}$ and $F: \mathcal{U} \rightarrow \mathfrak{g}$ be differentiable, such that $F(g \cdot X, g \cdot Y)=g \cdot F(X, Y)$ whenever $(X, Y) \in \mathcal{U}$ and $(g \cdot X, g \cdot Y) \in \mathcal{U}, g \in G$. Then

$$
\partial_{X} F(X, Y) \circ \operatorname{ad} X+\partial_{Y} F(X, Y) \circ \operatorname{ad} Y=\operatorname{ad} F(X, Y)
$$

as endomorphisms of $\mathfrak{g}$.

(b) Let $\mathcal{O}$ be an open subset of $\mathfrak{s} \times \mathfrak{s}$ and $F: \mathcal{O} \rightarrow \mathfrak{g}$ be differentiable, with $F(h \cdot X, h \cdot Y)=h \cdot F(X, Y)$ whenever $(X, Y) \in \mathcal{O}$ and $(h \cdot X, h \cdot Y) \in \mathcal{O}$, $h \in H$. Then

$$
\partial_{X} F(X, Y) \circ \operatorname{ad} X+\partial_{Y} F(X, Y) \circ \operatorname{ad} Y=\operatorname{ad} F(X, Y)
$$

as linear maps of $\mathfrak{h}$ into $\mathfrak{g}$.

Proof. (a) is Lemma 1.7.

(b) is proved similarly, looking at the derivative at $t=0$ of $F\left(e^{t V} \cdot X, e^{t V} \cdot Y\right)=$ $e^{t V} \cdot F(X, Y)$ with $V \in \mathfrak{h}$.

(i) $\Leftrightarrow$ (ii). We have $\left.\partial_{t} Z_{t}\right|_{t=1}=\left(\partial_{X} Z\right) X+\left(\partial_{Y} Z\right) Y-Z$ and (i) is equivalent to

$$
Z=\left(\partial_{X} Z\right)(X-x A)+\left(\partial_{Y} Z\right)(Y-y B),
$$

where everything is computed at $(X, Y)$. For $(X, Y) \in \mathcal{U}$ we have $2 Z \in \mathfrak{g}^{\prime}$ and, exp being a diffeomorphism on $\mathfrak{g}^{\prime}$, the map $\operatorname{sh} z / z=e^{z}\left(1-e^{-2 z}\right) / 2 z$ is an invertible endomorphism of $\mathfrak{g}$. We thus obtain an equivalent equality when applying it to both sides of (4.5). Since $f(x) X=f(0) X$ for any power series $f$ (and similarly with $Y$ or $Z$ ) the resulting equality is, in view of Proposition $4.2(v)$,

$$
Z(X, Y)=h \cdot((\operatorname{ch} y)(X+Y)-(\operatorname{ch} y \operatorname{sh} x+\operatorname{sh} y \operatorname{ch} x) A-(\operatorname{sh} y) C) .
$$

This is (ii) since $h^{-1} \cdot Z(X, Y)=Z(Y, X)$ by Proposition 4.2 (iii).

Before stating our next proposition we recall the first Kashiwara-Vergne equation (KV1): the Campbell-Hausdorff formula for the Lie algebra $\mathfrak{g}$ may be written as

$$
\frac{1}{2} \log \left(e^{2 Y} e^{2 X}\right)=X+Y-\left(1-e^{-2 x}\right) F(X, Y)-\left(e^{2 y}-1\right) G(X, Y),
$$


$F, G: \mathcal{U} \rightarrow \mathfrak{g}$ being analytic and given, in a neighborhood of the origin, by convergent series of Lie brackets of $X$ and $Y$. The existence of such Lie series is proved in 1.5.2.a. Note our slight change of notation in comparison with Section 1.3: if $F^{1}, G^{1}$ denote the functions we used in Chapter 1 we have $F(X, Y)=\frac{1}{2} F^{1}(2 X, 2 Y)$ and $G(X, Y)=\frac{1}{2} G^{1}(2 X, 2 Y)$ here. The present notation is more convenient in this chapter.

Proposition 4.5 (i) There exist two maps $A, C: \mathcal{U} \rightarrow[\mathfrak{g}, \mathfrak{g}]$, given by even Lie series of brackets of $X$ and $Y$, mapping the subset $\mathcal{O}=\mathcal{U} \cap(\mathfrak{s} \times \mathfrak{s})$ into $\mathfrak{h}_{*}=[\mathfrak{s}, \mathfrak{s}]$ and such that, for $(X, Y) \in \mathcal{U}$,

$$
Z(Y, X)=(\operatorname{ch} y)(X+Y)-(\operatorname{ch} y \operatorname{sh} x+\operatorname{sh} y \operatorname{ch} x) A(X, Y)-(\operatorname{sh} y) C(X, Y) .
$$

Besides the functions defined by $Z_{t}(X, Y):=t^{-1} Z(t X, t Y), A_{t}(X, Y):=$ $t^{-1} A(t X, t Y), C_{t}(X, Y):=t^{-1} C(t X, t Y)$ and $B_{t}:=A_{t}+C_{t}$ are analytic with respect to $(t, X, Y)$ on the open subset of $\mathbb{R} \times \mathfrak{g} \times \mathfrak{g}$ defined by $(t X, t Y) \in \mathcal{U}$. For $0 \leq t \leq 1,(X, Y) \in \mathcal{U}$,

$$
\begin{aligned}
\partial_{t} Z_{t} & =\left(\partial_{X} Z_{t}\right)\left[X, A_{t}\right]+\left(\partial_{Y} Z_{t}\right)\left[Y, B_{t}\right] \\
& =\left[Z_{t}, A_{t}\right]+\left(\partial_{Y} Z_{t}\right)\left[Y, C_{t}\right]
\end{aligned}
$$

(ii) If $F$ and $G$ are Lie series on $\mathcal{U}$ satisfying the Kashiwara-Vergne equation (KV1) one can take, for example,

$$
\begin{aligned}
& A(X, Y)=e^{-x} F(X, Y)+e^{x} F(-X,-Y), \\
& C(X, Y)=(G-F)(X, Y)+(G-F)(-X,-Y),
\end{aligned}
$$

and $B=A+C, B_{t}(X, Y)=t^{-1} B(t X, t Y)$.

Equation (4.6), restricted to $(X, Y) \in \mathcal{O}$, is the symmetric space analog of the Kashiwara-Vergne equation (KV1) for Lie groups. There is no analog of (KV2) in general; the $e$-function introduced below expresses the failure of this trace equality (see Proposition 4.19).

Proof. It suffices to prove (ii). According to Proposition 4.3 the main point is to prove (4.6). Let $F, G$ satisfy (KV1); then, for all $(X, Y) \in \mathcal{U}$,

$$
\begin{aligned}
Z(Y, X) & =\frac{1}{2} e^{-y} \log \left(e^{2 Y} e^{2 X}\right) \\
& =e^{-y}(X+Y)-\left(e^{y} e^{x}-e^{-y} e^{-x}\right) e^{-x} F-\left(e^{y}-e^{-y}\right)(G-F) \\
& =e^{-y}(X+Y)-2(\operatorname{ch} y \operatorname{sh} x+\operatorname{sh} y \operatorname{ch} x) e^{-x} F-2(\operatorname{sh} y)(G-F) .
\end{aligned}
$$

Changing signs of $X$ and $Y$ we get a similar expression of $Z(-Y,-X)=$ $-Z(Y, X)$ and the half difference is

$$
\begin{array}{r}
Z(Y, X)=(\operatorname{ch} y)(X+Y)-(\operatorname{ch} y \operatorname{sh} x+\operatorname{sh} y \operatorname{ch} x)\left(e^{-x} F+e^{x} F^{\vee}\right)- \\
-(\operatorname{sh} y)\left(G-F+G^{\vee}-F^{\vee}\right),
\end{array}
$$


with $F=F(X, Y), F^{\vee}=F(-X,-Y)$ and similarly for $G$. Choosing $A$ and $C$ as given by (4.9) and (4.10) we thus obtain (4.6).

Besides the Lie series $F$ begins as $F=\alpha X+\beta Y+\gamma[X, Y]+\cdots$ where $\alpha, \beta, \gamma$ are scalars, and (4.9) implies that $A$ is an even series of brackets of $X$ and $Y$ beginning as $A=2(\gamma-\beta)[X, Y]+\cdots$. Thus, for $(X, Y)$ near the origin, $A(X, Y)$ belongs to $[\mathfrak{g}, \mathfrak{g}]$. Let $\left(\lambda_{i}\right)$ be a finite family of linear forms on $\mathfrak{g}$ such that $\bigcap \operatorname{ker} \lambda_{i}=[\mathfrak{g}, \mathfrak{g}]$. By analytic continuation the functions $\lambda_{i} \circ A$ vanish identically on the connected open set $\mathcal{U}$, therefore $A$ maps $\mathcal{U}$ into $[\mathfrak{g}, \mathfrak{g}]$. Similarly $A$ maps $\mathcal{O}$ into $[\mathfrak{s}, \mathfrak{s}]$, and the same properties hold for $C$ and $B$.

By Proposition 4.3 our claim is proved for $\left.\partial_{t} Z_{t}\right|_{t=1}$ and, replacing $(X, Y)$ by $(t X, t Y)$, we obtain $(4.7)(4.8)$.

Remarks. (a) If $(F, G)$ is an $\alpha$ - and $\beta$-invariant solution of (KV1) (see Section 1.7), Proposition 1.13 shows that (with the suitable factors 2 included)

$$
\begin{aligned}
2 e^{-x} F(X, Y) & =H(2 X, 2 Y)+\frac{1}{4} e^{-x} \log \left(e^{2 X} e^{2 Y}\right)-\frac{1}{2} X \\
& =H(2 X, 2 Y)+\frac{1}{2}(Z(X, Y)-X)
\end{aligned}
$$

where $H$ is an even Lie series. The function $A$ of (4.9) is then $A(X, Y)=$ $H(2 X, 2 Y)$ since $Z(X, Y)-X$ is odd (Proposition 4.2).

(b) Any solution $(A, C)$ of (4.6) can be replaced by the even solution

$$
\left(\frac{1}{2}\left(A+A^{\vee}\right), \frac{1}{2}\left(C+C^{\vee}\right)\right)
$$

since $Z(Y, X)-\operatorname{ch} y(X+Y)$ is odd.

\subsection{4 $a, b$ and $\Phi$}

With the above tools we can now construct a diffeomorphism $\Phi$ transforming the map $Z$ into its flat analog $Z_{0}(X, Y)=X+Y$. As before dots denote the adjoint action.

Theorem 4.6 There exists a connected open neighborhood $\Omega$ of the origin in $\mathfrak{s} \times \mathfrak{s}$, contained in $\mathcal{O}$ and having the same invariance properties as $\mathcal{O}$ (Lemma 4.1), and an analytic diffeomorphism $\Phi$ of $\Omega$ onto $\Phi(\Omega) \subset \mathcal{O}$ endowed with the following properties:

(i) $\Phi(X, Y)=(a \cdot X, b \cdot Y)$ where $a=a(X, Y)$ and $b=b(X, Y)$ are given by even analytic maps from $\Omega$ into the Lie subgroup $H_{*}$ of $H$ with Lie algebra $\mathfrak{h}_{*}=[\mathfrak{s}, \mathfrak{s}]$.

(ii) $\Phi(-X,-Y)=-\Phi(X, Y), \Phi(h \cdot(X, Y))=h \cdot \Phi(X, Y)$ (diagonal adjoint action of $H)$ for all $(X, Y) \in \Omega, h \in H$.

(iii) If $(X, Y) \in \Omega$ and $[X, Y]=0$, then $a(X, Y)=b(X, Y)=e$ and $\Phi(X, Y)=(X, Y)$. 
(iv) $(Z \circ \Phi)(X, Y)=X+Y$ for all $(X, Y) \in \Omega$.

(v) Assume $H$ is compact and $\operatorname{Exp}: \mathfrak{s} \rightarrow S$ is a global diffeomorphism. Then the above is valid on $\Omega=\mathfrak{s} \times \mathfrak{s}$ and $\Phi$ is a global diffeomorphism of $\mathfrak{s} \times \mathfrak{s}$ onto itself.

For later reference we collect here a few facts taken from the proof below. The diffeomorphism $\Phi$ is obtained from maps $A_{t}, B_{t}: \mathcal{O} \rightarrow \mathfrak{h}_{*}$ chosen according to Proposition 4.5 ( $i$ ) (for example those given by (4.9)(4.10)), and taking $a=a_{1}, b=b_{1}, \Phi=\Phi_{1}$ where $a_{t}, b_{t}, \Phi_{t}$ solve, for $0 \leq t \leq 1$, the differential equations

$$
\begin{gathered}
\left\{\begin{array}{c}
\partial_{t} a_{t}(X, Y)=\left(D_{e} R_{a_{t}}\right) A_{t}\left(a_{t} \cdot X, b_{t} \cdot Y\right), a_{0}(X, Y)=e \\
\partial_{t} b_{t}(X, Y)=\left(D_{e} R_{b_{t}}\right) B_{t}\left(a_{t} \cdot X, b_{t} \cdot Y\right), b_{0}(X, Y)=e
\end{array}\right. \\
\Phi_{t}(X, Y)=\left(X_{t}, Y_{t}\right)=\left(a_{t} \cdot X, b_{t} \cdot Y\right) \\
\left\{\begin{array}{l}
\partial_{t} X_{t}=\left[A_{t}\left(X_{t}, Y_{t}\right), X_{t}\right], X_{0}=X \\
\partial_{t} Y_{t}=\left[B_{t}\left(X_{t}, Y_{t}\right), Y_{t}\right], Y_{0}=Y \\
\Phi_{0}=I d, \Phi_{1}=\Phi .
\end{array}\right.
\end{gathered}
$$

Besides, for all $(X, Y) \in \Omega, t \in[0,1], h \in H$,

$$
\begin{gathered}
a_{t}(h \cdot X, h \cdot Y)=h a_{t}(X, Y) h^{-1}, b_{t}(h \cdot X, h \cdot Y)=h b_{t}(X, Y) h^{-1} \\
a_{t}(X, Y)=a(t X, t Y), b_{t}(X, Y)=b(t X, t Y) \\
\Phi_{t}(X, Y)=t^{-1} \Phi(t X, t Y),\left(Z_{t} \circ \Phi_{t}\right)(X, Y)=X+Y .
\end{gathered}
$$

The Jacobian of $\Phi_{t}$ is positive and given by

$$
\begin{aligned}
\partial_{t} \log \operatorname{det}_{\mathfrak{s} \times \mathfrak{s}} D \Phi_{t}(X, Y) & = \\
& =\operatorname{tr}_{\mathfrak{s}}\left(\operatorname{ad}\left(A_{t}+B_{t}\right)-x \circ \partial_{X} A_{t}-y \circ \partial_{Y} B_{t}\right) \circ \Phi_{t}(X, Y) .
\end{aligned}
$$

Proof. (i) and (ii) Let us consider the differential system (4.11) on the manifold $H_{*} \times H_{*}$, with parameters $(X, Y)$. The functions $A_{t}$ and $B_{t}$ are analytic with respect to $(t, X, Y)$ in the open neighborhood of $[0,1] \times \mathcal{O}$ in $\mathbb{R} \times \mathfrak{s} \times \mathfrak{s}$ defined by $(t X, t Y) \in \mathcal{O}$, and their values belong to $\mathfrak{h}_{*}$. It follows that there exist $\varepsilon>0$, an open subset $\omega$ of $\mathcal{O}$, which may be assumed invariant under $(X, Y) \mapsto(t X, t Y)$ for $-1 \leq t \leq 1$ and $(X, Y) \mapsto(Y, X)$, and two unique analytic functions $a_{t}(X, Y), b_{t}(X, Y) \in H_{*}$, solutions of (4.11) for $|t| \leq \varepsilon$ and $(X, Y) \in \omega$. In particular $\left(t a_{t} \cdot X, t b_{t} \cdot Y\right)$ belongs to $\mathcal{O}$ at such points. From the uniqueness and the properties of $A_{t}, B_{t}$ we infer that

$$
a_{t}(-X,-Y)=a_{t}(X, Y), a_{t}(s X, s Y)=a_{s t}(X, Y)
$$

for $(X, Y) \in \omega,|t| \leq \varepsilon, 0 \leq s \leq 1$, and the same for $b_{t}$. Replacing $\omega$ by $\varepsilon \omega$ we can therefore assume $\varepsilon=1$. Likewise

$$
a_{t}(h \cdot X, h \cdot Y)=h a_{t}(X, Y) h^{-1}
$$


for $h \in H$, whenever $(X, Y)$ and $(h \cdot X, h \cdot Y)$ belong to $\omega$, and the same for $b_{t}$. We can therefore replace $\omega$ by $\Omega:=H \cdot \omega$ (diagonal adjoint action of $H$ on $\mathfrak{s} \times \mathfrak{s}$ ). This set $\Omega$ is star-shaped, therefore connected, and meets all our requirements. Our $a_{t}, b_{t}$ extend to $\Omega$ as solutions of (4.11) up to $t=1$; they still belong to $H_{*}$ since $H_{*}$ is a normal subgroup of $H$.

Now $X_{t}:=a_{t} \cdot X, Y_{t}:=b_{t} \cdot Y$ satisfy the differential equations (4.13) for $0 \leq t \leq 1$, with initial conditions $\left(X_{0}, Y_{0}\right)=(X, Y) \in \Omega$. By the general theory of time-dependent vector fields the map $\Phi_{t}:(X, Y) \mapsto\left(X_{t}, Y_{t}\right)$ is an analytic diffeomorphism of $\Omega$ onto the open set $\Phi_{t}(\Omega)$. As noted above $\left(t X_{t}, t Y_{t}\right)$ belongs to $\mathcal{O}$ for $0 \leq t \leq 1$. Thus, writing $\Phi$ instead of $\Phi_{1}$, we see that $\Phi(\Omega)$ is an open subset of $\mathcal{O}$ and $\Phi$ satisfies (i) and (ii) in the theorem. (iii) If $(X, Y) \in \Omega$ and $[X, Y]=0$ we have $A(X, Y)=B(X, Y)=0$, since $A$ and $B$ are series of brackets of $X$ and $Y$. It follows that $a_{t}(X, Y)=$ $b_{t}(X, Y)=e$ is the (unique) solution of $(4.11)$, whence $\Phi(X, Y)=(X, Y)$ in this case.

(iv) By (4.13) and Proposition 4.5 we have

$$
\partial_{t}\left(Z_{t}\left(X_{t}, Y_{t}\right)\right)=\left(\partial_{t} Z_{t}\right)\left(X_{t}, Y_{t}\right)+\left(\partial_{X} Z_{t}\right) \partial_{t} X_{t}+\left(\partial_{Y} Z_{t}\right) \partial_{t} Y_{t}=0,
$$

therefore $Z_{t}\left(X_{t}, Y_{t}\right)=Z_{0}(X, Y)=X+Y$ for $0 \leq t \leq 1$.

Finally $\partial_{t} \log \operatorname{det} \Phi_{t}(X, Y)$ is the divergence, computed at $\left(X_{t}, Y_{t}\right)$, of the vector field $\left(\left[A_{t}(X, Y), X\right],\left[B_{t}(X, Y), Y\right]\right) \in \mathfrak{s} \times \mathfrak{s}$ defining the flow $\Phi_{t}$, that is

$$
\begin{aligned}
\operatorname{tr}_{\mathfrak{s} \times \mathfrak{s}}\left(\begin{array}{cc}
\operatorname{ad} A_{t}-x \partial_{X} A_{t} & -x \partial_{Y} A_{t} \\
-y \partial_{X} B_{t} & \operatorname{ad} B_{t}-y \partial_{Y} B_{t}
\end{array}\right)\left(X_{t}, Y_{t}\right)= \\
\quad=\operatorname{tr}_{\mathfrak{s}}\left(\operatorname{ad}\left(A_{t}+B_{t}\right)-x \circ \partial_{X} A_{t}-y \circ \partial_{Y} B_{t}\right) \circ \Phi_{t}(X, Y),
\end{aligned}
$$

and (4.17) is proved.

(v) If Exp is a global diffeomorhism we may take $\mathcal{O}=\mathfrak{s} \times \mathfrak{s}$ (as noted before Lemma 4.1) and $A_{t}, B_{t}$ are analytic functions of $(t, X, Y)$ in $\mathbb{R} \times \mathfrak{s} \times \mathfrak{s}$. Furthermore, if $H$ is compact, $\left(\left(D_{e} R_{a_{t}}\right) A_{t}\left(a_{t} \cdot X, b_{t} \cdot Y\right),\left(D_{e} R_{b_{t}}\right) B_{t}\left(a_{t} \cdot X, b_{t} \cdot Y\right)\right)$ is an analytic time-dependent vector field on the compact manifold $H \times H$ for any given $(X, Y) \in \mathfrak{s} \times \mathfrak{s}$. It follows that $a_{t}, b_{t}$ and $\Phi_{t}$ are globally defined and analytic on $\mathbb{R} \times \mathfrak{s} \times \mathfrak{s}$. Moreover, if $\Phi(X, Y)=(a \cdot X, b \cdot Y)$ belongs to a compact subset $K_{1} \times K_{2}$ of $\mathfrak{s} \times \mathfrak{s}$, then $(X, Y)$ belongs to the compact set $\left(H \cdot K_{1}\right) \times\left(H \cdot K_{2}\right)$. Thus $\Phi$ is a proper mapping of $\mathfrak{s} \times \mathfrak{s}$ into itself, hence a global diffeomorphism onto by Hadamard's theorem.

\subsection{5 $c, h$ and $\Phi$}

Keeping to the notation of Theorem 4.6, we now study

$$
c_{t}(X, Y):=a_{t}(X, Y)^{-1} b_{t}(X, Y) \in H .
$$

A remarkable symmetry of our $e$-function (Theorem 4.24) will follow from the properties of $c_{t}$. 
In order to lighten notation in all proofs of this section we shall compute as in a matrix Lie group, writing e.g. $\left(\partial_{t} a_{t}\right) c_{t}$ rather than $\left(D_{a_{t}} R_{c_{t}}\right) \partial_{t} a_{t}$, and sometimes $a X a^{-1}$ for $a \cdot X=\operatorname{Ad} a(X)$.

We first show that $c_{t}$ can be obtained from a simple differential equation.

Lemma 4.7 Let $C(X, Y)=B(X, Y)-A(X, Y), C_{t}(X, Y)=t^{-1} C(t X, t Y)$. Then $c_{t}=c_{t}(X, Y)$ is given by the differential equation, for $(X, Y) \in \Omega$, $0 \leq t \leq 1$,

$$
\partial_{t} c_{t}=\left(D_{e} R_{c_{t}}\right) C_{t}\left(X, c_{t} \cdot Y\right), c_{0}=e
$$

and $U_{t}:=c_{t} \cdot Y$ by

$$
\partial_{t} U_{t}=\left[C_{t}\left(X, U_{t}\right), U_{t}\right], U_{0}=Y .
$$

Proof. From $a_{t} c_{t}=b_{t}$ it follows that (in our simplified notation)

$$
a_{t}\left(\partial_{t} c_{t}\right)+\left(\partial_{t} a_{t}\right) c_{t}=\partial_{t} b_{t}
$$

that is, with (4.11),

$$
a_{t}\left(\partial_{t} c_{t}\right)+A_{t}\left(a_{t} \cdot X, b_{t} \cdot Y\right) a_{t} c_{t}=B_{t}\left(a_{t} \cdot X, b_{t} \cdot Y\right) b_{t} .
$$

Therefore $a_{t}\left(\partial_{t} c_{t}\right)=C_{t}\left(a_{t} \cdot X, b_{t} \cdot Y\right) b_{t}$ and (4.19) follows from the $H$ equivariance of $A$ and $B$, which implies $C_{t}\left(a_{t} \cdot X, b_{t} \cdot Y\right)=a_{t} C_{t}\left(X, c_{t} \cdot Y\right) a_{t}^{-1}$. Then $U_{t}=c_{t} Y c_{t}^{-1}$ gives

$$
\begin{aligned}
\partial_{t} U_{t} & =\left(\partial_{t} c_{t}\right) Y c_{t}^{-1}-c_{t} Y c_{t}^{-1}\left(\partial_{t} c_{t}\right) c_{t}^{-1} \\
& =\left[\left(\partial_{t} c_{t}\right) c_{t}^{-1}, U_{t}\right]=\left[C_{t}\left(X, U_{t}\right), U_{t}\right] .
\end{aligned}
$$

Remark. The solution $\left(a_{t}, b_{t}\right)$ of the system (4.11) can thus be obtained from $A$ and $C$ by solving (4.19) for $c_{t}$ first, then solving

$$
\partial_{t} a_{t}=\left(D_{e} L_{a_{t}}\right) A_{t}\left(X, c_{t} \cdot Y\right), a_{0}=e
$$

whence $b_{t}=a_{t} c_{t}$ finally.

Proposition 4.8 (i) For $(X, Y) \in \Omega$ let $c(X, Y)=a(X, Y)^{-1} b(X, Y)$. Then $c(X, Y)$ belongs to $H_{*}$ and $c_{t}(X, Y)=c(t X, t Y)$ for $t \in[0,1]$. Besides

$$
Z(X, c(X, Y) \cdot Y)=a(X, Y)^{-1} \cdot(X+Y), c(h \cdot X, h \cdot Y)=h c(X, Y) h^{-1}
$$

for any $h \in H$, and $c(X, Y)=e$ whenever $[X, Y]=0$.

(ii) Assume $F, G$ satisfy (KV1) with $G(X, Y)=F(-Y,-X)$, and let $C$ be defined by (4.10). Then $C(X, Y)=C(-X,-Y)=-C(Y, X)$ and

$$
c(X, Y)=c(-X,-Y)=c(Y, X)^{-1} .
$$


By Proposition 1.12 we can always assume that $F$ and $G$ satisfy the condition in (ii) ( $\alpha$-invariance).

Proof. (i) is immediate from Theorem 4.6 and (4.14), (4.15) for $a$ and $b$. (ii) We have $C(X, Y)=F(-Y,-X)-F(X, Y)+F(Y, X)-F(-X,-Y)$ by (4.10) and the assumption on $F$ and $G$, therefore $C(X, Y)=C(-X,-Y)=$ $-C(Y, X)$. The equality $c(X, Y)=c(-X,-Y)$ now follows from uniqueness of solutions of the differential equation (4.19). Likewise, letting $\bar{c}_{t}=c_{t}(Y, X)$ we have $\bar{c}_{0}=e$ and

$$
\begin{aligned}
\partial_{t}\left(\bar{c}_{t}^{-1}\right) & =-\bar{c}_{t}^{-1}\left(\partial_{t} \bar{c}_{t}\right) \bar{c}_{t}^{-1}=-\bar{c}_{t}^{-1} C_{t}\left(Y, \bar{c}_{t} \cdot X\right) \\
& =C_{t}\left(X, \bar{c}_{t}^{-1} \cdot Y\right) \bar{c}_{t}^{-1}
\end{aligned}
$$

in view of the skew symmetry and $H$-equivariance of $C_{t}$. Thus $\bar{c}_{t}^{-1}=$ $c_{t}(X, Y)$ by uniqueness.

The function $c(X, Y)$ is closely related to the $e$-function constructed below: see Propositions 4.18 and 4.19; see also Corollary 3.17 above, with an example for the 2-dimensional hyperbolic space.

The next proposition will be useful when extending our $e$-function to line bundles, with the $H$-component $h(X, Y)$ defined by $e^{X} e^{Y}=e^{Z(X, Y)} h(X, Y)$ playing a significant role in that case. Let us begin with a lemma.

Lemma 4.9 For $(X, Y) \in \mathcal{U}$ let $A=A(X, Y), \bar{A}=A(Y, X)$ and similarly for other functions. If $A$ and $C$ in (4.9)(4.10) arise from $F, G$ satisfying (KV1) and $G(X, Y)=F(-Y,-X)$, then

$$
\begin{aligned}
& \frac{1}{4}\left(\log e^{2 Y} e^{2 X}+\log e^{2 X} e^{2 Y}\right)=(\operatorname{ch} y) \bar{Z}=X+Y-(\operatorname{sh} x) A-(\operatorname{sh} y) \bar{A} \\
& \frac{1}{4}\left(\log e^{2 Y} e^{2 X}-\log e^{2 X} e^{2 Y}\right)=(\operatorname{sh} y) \bar{Z}=C+(\operatorname{ch} x) A-(\operatorname{ch} y) \bar{A}
\end{aligned}
$$

Proof. By (4.9) and the symmetry assumption we have $A=e^{-x} F+e^{x} \bar{G}$ hence

$$
(\operatorname{sh} x) A=\frac{1}{2}\left(1-e^{-2 x}\right) F+\frac{1}{2}\left(e^{2 x}-1\right) \bar{G} .
$$

Permuting $X$ and $Y$ and adding, we obtain (4.21) in view of the KashiwaraVergne equality (KV1). The expression with $\bar{Z}$ follows from Proposition 4.2. Likewise

$$
(\operatorname{ch} x) A=F+\bar{G}-\frac{1}{2}\left(1-e^{-2 x}\right) F+\frac{1}{2}\left(e^{2 x}-1\right) \bar{G} .
$$

Permuting $X$ and $Y$, then combining with $C=G-F+\bar{F}-\bar{G}$ we obtain (4.22).

Remark. With $V=\frac{1}{2} \log e^{2 X} e^{2 Y}$ equation (4.22) may be rewritten as

$$
C=\overline{\left((\operatorname{ch} x) A+\frac{1}{2} V\right)}-\left((\operatorname{ch} x) A+\frac{1}{2} V\right) .
$$


Proposition 4.10 Assume $F, G$ satisfy $(K V 1)$ and $G(X, Y)=F(-Y,-X)$. Let $\Phi$ be constructed by means of $A, C$ defined by (4.9), (4.10) and let $\widehat{h}:=$ $h \circ \Phi$. Then, for all $(X, Y) \in \Omega$,

(i) $\widehat{h}(X, Y)=a(Y, X) b(X, Y)^{-1}=b(Y, X) a(X, Y)^{-1}, \widehat{h}(Y, X)=\widehat{h}(X, Y)^{-1}$.

(ii) Let $\left(X_{1}, Y_{1}\right):=\Phi(X, Y)$. Then $\Phi(Y, X)=\widehat{h}(X, Y) \cdot\left(Y_{1}, X_{1}\right)$ (diagonal adjoint action of $H$ on $\mathfrak{s} \times \mathfrak{s})$.

(iii) $e^{X} c(X, Y) e^{Y}=a(X, Y)^{-1} e^{X+Y} a(Y, X)$, and $e^{X} e^{c(X, Y) \cdot Y}=a(X, Y)^{-1} e^{X+Y} b(Y, X)$.

The latter equation improves $Z(X, c(X, Y) \cdot Y)=a(X, Y)^{-1} \cdot(X+Y)$ given by Proposition 4.8 .

Proof. (i) and (ii). Let $a_{t}=a_{t}(X, Y), \bar{a}_{t}=a_{t}(Y, X)$ (and similar notation for other functions) and $k_{t}:=\bar{a}_{t} b_{t}^{-1}$. The symmetry $\bar{c}_{t}=c_{t}^{-1}$ (Proposition $4.8(i i))$ gives $k_{t}=\bar{b}_{t} a_{t}^{-1}=\bar{k}_{t}^{-1}$. Besides

$$
\Phi_{t}(Y, X)=\left(\bar{a}_{t} \cdot Y, \bar{b}_{t} \cdot X\right)=\left(k_{t} b_{t} \cdot Y, k_{t} a_{t} \cdot X\right)=k_{t} \cdot\left(Y_{t}, X_{t}\right) .
$$

Thus (i) and (ii) will follow from the equality $k_{t}=\widehat{h}_{t}$ with $\widehat{h}_{t}(X, Y):=$ $\widehat{h}(t X, t Y)=h\left(t X_{t}, t Y_{t}\right)$. Let us prove that $k_{t}$ and $\widehat{h}_{t}$ solve the same differential equation.

On the one hand

$$
k_{t}^{-1} \partial_{t} k_{t}=k_{t}^{-1}\left(\partial_{t} \bar{a}_{t}\right) b_{t}^{-1}-\left(\partial_{t} b_{t}\right) b_{t}^{-1} .
$$

But $\left(\partial_{t} a_{t}\right) a_{t}^{-1}=A_{t}\left(\Phi_{t}(X, Y)\right)$ and $\left(\partial_{t} b_{t}\right) b_{t}^{-1}=B_{t}\left(\Phi_{t}(X, Y)\right)$ by (4.11), hence

$$
\begin{aligned}
\left(\partial_{t} \bar{a}_{t}\right) \bar{a}_{t}^{-1} & =A_{t}\left(\Phi_{t}(Y, X)\right)=A_{t}\left(k_{t} \cdot Y_{t}, k_{t} \cdot X_{t}\right) \\
& =\bar{A}_{t}\left(k_{t} \cdot X_{t}, k_{t} \cdot Y_{t}\right)=k_{t} \bar{A}_{t}\left(\Phi_{t}(X, Y)\right) k_{t}^{-1}
\end{aligned}
$$

and finally

$$
k_{t}^{-1} \partial_{t} k_{t}=\left(\bar{A}_{t}-B_{t}\right) \circ \Phi_{t} .
$$

On the other hand, replacing $(X, Y)$ by $\left(t X_{t}, t Y_{t}\right)$ in the definition of $h(X, Y)$ we have

$$
\widehat{h}_{t}(X, Y)=e^{-t(X+Y)} e^{t X_{t}} e^{t Y_{t}} .
$$

Now $\partial_{t}\left(t X_{t}\right)=X_{t}-t x_{t}\left(A_{t} \circ \Phi_{t}\right), \partial_{t}\left(t Y_{t}\right)=Y_{t}-t y_{t}\left(B_{t} \circ \Phi_{t}\right)$ by (4.13), with $x_{t}=\operatorname{ad} X_{t}, y_{t}=\operatorname{ad} Y_{t}$; therefore, remembering the differential of exp,

$$
\begin{aligned}
\partial_{t}\left(e^{t X_{t}}\right) & =e^{t X_{t}}\left(X_{t}+\left(e^{-t x_{t}}-1\right)\left(A_{t} \circ \Phi_{t}\right)\right) \\
\partial_{t}\left(e^{t Y_{t}}\right) & =e^{t Y_{t}}\left(Y_{t}+\left(e^{-t y_{t}}-1\right)\left(B_{t} \circ \Phi_{t}\right)\right) .
\end{aligned}
$$

It follows that, at $(X, Y)$,

$$
\begin{aligned}
\widehat{h}_{t}^{-1} \partial_{t} \widehat{h}_{t}=-\widehat{h}_{t}^{-1} & \cdot(X+Y)+ \\
& +\left[e^{-t y}\left(X+\left(e^{-t x}-1\right) A_{t}\right)+Y+\left(e^{-t y}-1\right) B_{t}\right] \circ \Phi_{t} .
\end{aligned}
$$


The left-hand side and $A_{t}, B_{t}$ belong to $\mathfrak{h}$, whereas the first term on the right belongs to $\mathfrak{s}$. Writing $e^{-t x}=\operatorname{ch} t x-\operatorname{sh} t x$ etc. the $\mathfrak{h}$-components separate as

$$
\begin{aligned}
\widehat{h}_{t}^{-1} \partial_{t} \widehat{h}_{t} & =H_{t} \circ \Phi_{t}, \text { with } H_{t}(X, Y)=t^{-1} H(t X, t Y) \text { and } \\
H(X, Y) & =-(\operatorname{sh} y) X+(\operatorname{ch} y \operatorname{ch} x+\operatorname{sh} y \operatorname{sh} x) A(X, Y)+ \\
& +(\operatorname{ch} y) C(X, Y)-B(X, Y),
\end{aligned}
$$

with $C=B-A$ as above. Now the symmetry assumption $G(X, Y)=$ $F(-Y,-X)$ and Lemma 4.9 imply the simpler expression $H=\bar{A}-B$ : indeed, to eliminate $\bar{Z}$ between (4.21) and (4.22) we may apply $\operatorname{sh} y$, resp. $\operatorname{ch} y$, to both sides of these equalities and substract ${ }^{1}$. We infer that $k_{t}=\widehat{h}_{t}$, which proves (i) and (ii).

(iii) Replacing $(X, Y)$ by $\Phi(X, Y)=(a \cdot X, b \cdot Y)$ in the equality $e^{X} e^{Y}=e^{Z} h$ we obtain

$$
e^{a \cdot X} e^{b \cdot Y}=e^{X+Y} \bar{a} b^{-1}
$$

since $Z \circ \Phi(X, Y)=X+Y$ and $h \circ \Phi(X, Y)=\bar{a} b^{-1}$ by (i) above and Theorem $4.6(i v)$. Writing $e^{a \cdot X}=a e^{X} a^{-1}$ etc. our claim follows with $c=$ $a^{-1} b=\bar{b}^{-1} \bar{a}$.

\subsection{Campbell-Hausdorff e-Functions}

\subsubsection{An Auxiliary Lemma}

Terms like $\operatorname{det} \operatorname{Ad}(\ldots)$ or $\operatorname{trad}(\ldots)$ will frequently occur in the sequel and it will be useful to keep in mind the simplifications provided, on many examples, by the following lemma. As before $H_{*}$ is the (connected) Lie subgroup of $H$ with Lie algebra $\mathfrak{h}_{*}=[\mathfrak{s}, \mathfrak{s}] \subset \mathfrak{h}$.

Lemma 4.11 Let $G / H$ be a symmetric space. The following properties (i) to (v) are equivalent:

(i) $\operatorname{tr}_{\mathfrak{h}} \operatorname{ad} \mathfrak{h}_{*}=0$

(ii) $\operatorname{tr}_{\mathfrak{s}}$ ad $\mathfrak{h}_{*}=0$

(iii) the character $\operatorname{tr}_{\mathfrak{h}}$ ad of $\mathfrak{h}$ extends to a character of the Lie algebra $\mathfrak{g}$

(iv) $\operatorname{det}_{\mathfrak{h}} \operatorname{Ad} h=1$ for all $h \in H_{*}$

(v) $\operatorname{det}_{\mathfrak{s}} \operatorname{Ad} h=1$ for all $h \in H_{*}$

Property (vi) implies (i) to (v), and is equivalent to them if $G$ is simply connected:

(vi) $G / H$ has a $G$-semi-invariant measure (i.e. $G$-invariant up to some factor, which is a character of the group $G$ ).

By Theorem 4.24 (ii) below properties (i) to (v) will also be equivalent to the symmetry of our $e$-function. They hold true in particular if $G / H$ has a

\footnotetext{
${ }^{1}$ Applying $\operatorname{ch} y$, resp. $\operatorname{sh} y$, instead and adding eliminates $\bar{A}$ and gives back the expression (4.6) of $\bar{Z}$.
} 
$G$-invariant measure (which is equivalent, for $H$ connected, to $\operatorname{det}_{\mathfrak{s}} \operatorname{Ad} h=1$ for all $h \in H$ ), e.g. if $\operatorname{Ad} H$ is a compact group, a fortiori if $H$ is compact.

Proof. (i) $\Leftrightarrow$ (ii) because $\operatorname{tr}_{\mathfrak{h}} \operatorname{ad}[X, Y]+\operatorname{tr}_{\mathfrak{s}} \operatorname{ad}[X, Y]=\operatorname{tr}_{\mathfrak{g}} \operatorname{ad}[X, Y]=0$ for $X, Y \in \mathfrak{s}$.

(iii) $\Rightarrow($ i $)$. A character of $\mathfrak{g}$ is a linear form $f$ on $\mathfrak{g}$ such that $f([\mathfrak{g}, \mathfrak{g}])=0$; a fortiori $f([\mathfrak{s}, \mathfrak{s}])=0$, hence (i) if $f$ extends $\operatorname{tr}_{\mathfrak{h}}$ ad.

(i) $\Rightarrow$ (iii). If (i) holds $\operatorname{tr}_{\mathfrak{h}}$ ad can be extended (by 0 on $\mathfrak{s}$ ) to a character of $\mathfrak{g}=\mathfrak{h} \oplus \mathfrak{s}$, since $[\mathfrak{h}, \mathfrak{s}] \subset \mathfrak{s}$ and $[\mathfrak{s}, \mathfrak{s}]=\mathfrak{h}_{*}$.

(i) $\Leftrightarrow(i v)$ and $(i i) \Leftrightarrow(v)$, because $\operatorname{det} \operatorname{Ad}\left(e^{X}\right)=e^{\operatorname{trad} X}$ and $\exp \mathfrak{h}_{*}$ generates $H_{*}$.

(vi) $\Leftrightarrow$ (iii). Indeed the space $G / H$ has a $G$-semi-invariant measure ${ }^{2}$ with multiplier $\chi$ if and only if $\chi(h)=\Delta_{H}(h) / \Delta_{G}(h)$ for all $h \in H$, where $\Delta_{H}$, resp. $\Delta_{G}$, is the modular function of the group $H$, resp. $G$, and such a measure exists if and only if $\Delta_{H}$ extends to a character of $G$. Since $\Delta_{H}\left(e^{X}\right)=$ $e^{\operatorname{tr}_{\mathfrak{h}} \text { ad } X}$ for $X \in \mathfrak{h}$ the latter property implies (iii), and is equivalent to it if $G$ is simply connected.

\subsubsection{Construction of an $e$-Function}

Let us go back to the transfer map of Definition 3.2 associated to a factor $j$ (analytic, strictly positive and $H$-invariant on $\mathfrak{s}^{\prime}$, with $j(0)=1$ ). With the tools $a, b, \Phi$ from Section 4.2.4 we shall now construct an $e$-function (Definition 3.3) on an arbitrary symmetric space.

In order to motivate the definition of $e$ in the next theorem let us consider first the simple case of a Riemannian symmetric space, with $H$ compact, assuming here $j=J^{1 / 2}$ so that $\sim$ agrees for functions and distributions, with $(u(X) d X)^{\sim}=\widetilde{u}(x) d x$ (see Example 1 in 3.1.1). If $u, v$ are $H$-invariant functions on $\mathfrak{s}$ (with supports in a neighborhood of the origin) we have, for $f \in \mathcal{D}\left(\mathfrak{s}^{\prime}\right)$,

$$
\begin{gathered}
\int_{S}\left(\widetilde{u} *_{S} \widetilde{v}\right)(x) \widetilde{f}(x) d x=\int_{S \times S} \widetilde{u}(g H) \widetilde{v}\left(g^{\prime} H\right) \widetilde{f}\left(g g^{\prime} H\right) d(g H) d\left(g^{\prime} H\right) \\
=\int_{\mathfrak{s} \times \mathfrak{s}} \widetilde{u}(\operatorname{Exp} X) \widetilde{v}(\operatorname{Exp} Y) \widetilde{f}(\operatorname{Exp} Z(X, Y)) J(X) J(Y) d X d Y \\
=\int_{\mathfrak{s} \times \mathfrak{s}} u(X) v(Y)\left(\frac{J(X) J(Y)}{J(Z(X, Y))}\right)^{1 / 2} f(Z(X, Y)) d X d Y .
\end{gathered}
$$

Replacing $(X, Y)$ by $\Phi(X, Y)=(a \cdot X, b \cdot Y)$ and remembering the $H$ invariance of $u, v$ and $J$, the integral becomes

$$
\begin{aligned}
\int_{S}\left(\widetilde{u} *_{S} \widetilde{v}\right)(x) \widetilde{f}(x) d x & =\int_{\mathfrak{s} \times \mathfrak{s}} u(X) v(Y) e(X, Y) f(X+Y) d X d Y \\
\text { with } e(X, Y) & : \quad=\left(\frac{J(X) J(Y)}{J(X+Y)}\right)^{1 / 2} \operatorname{det}_{\mathfrak{s} \times \mathfrak{s}} D \Phi(X, Y)
\end{aligned}
$$

${ }^{2}$ See e.g. Bourbaki, Intégration, chap. VII, $\S 2 \mathrm{n}^{\circ} 6$. 
(remembering that the Jacobian of $\Phi$ is positive). This equality generalizes as follows.

Theorem 4.12 Let $S=G / H$ be a (simply connected) symmetric space and $\mathfrak{g}=\mathfrak{h} \oplus \mathfrak{s}$ the corresponding decomposition of the Lie algebra of $G$. Let $u$, $v$, be $H$-invariant distributions on open subsets of $\mathfrak{s}$ and let $f \in C^{\infty}(\mathfrak{s})$, with suitable supports. Then

$$
\left\langle\widetilde{u} *_{S} \widetilde{v}, \tilde{f}\right\rangle=\langle u(X) \otimes v(Y), e(X, Y) f(X+Y)\rangle
$$

with

$$
e(X, Y):=\frac{j(X) j(Y)}{j(X+Y)} \operatorname{det}_{\mathfrak{s} \times \mathfrak{s}} D \Phi(X, Y)\left(\operatorname{det}_{\mathfrak{s}} \operatorname{Ad} a(X, Y) b(X, Y)\right)^{-1} .
$$

Formula (4.23) defines an e-function for $S=G / H$ associated with $j$ and arising from $A, B$ as introduced in Proposition 4.5 (i). It is analytic and strictly positive on $\Omega$.

The convolution equality holds in particular if $u, v$ are $H$-invariant distributions on open subsets of $\mathfrak{s}$, with $\operatorname{supp} v=\{0\}, f \in C^{\infty}(\mathfrak{s})$, and $\operatorname{supp} u \cap$ supp $f \cap \Omega_{1}$ is compact.

Here $\Omega_{1}=\{X \in \mathfrak{s} \mid(X, 0) \in \Omega\}$. For instance, the latter assumption holds true if $U$ is open in $\mathfrak{s}, u \in \mathcal{D}^{\prime}(U)^{H}$ and $f \in \mathcal{D}\left(\Omega_{1} \cap U\right)$. A more general assumption on supports will be specified at the end of the proof, but the special case mentioned in the theorem suffices for the application to invariant differential operators. We refer to (4.23) as a Campbell-Hausdorff $\boldsymbol{e}-$ function. More precisely:

Definition 4.13 We shall call (4.23) the $(j, \Phi)$ - function of $S$. If $\Phi$ is defined by means of $A, B$ chosen according to Proposition 4.5 (ii), with $F, G$ satisfying the Kashiwara-Vergne equation (KV1), we shall call (4.23) the $(\boldsymbol{j}, \boldsymbol{F}, \boldsymbol{G})$ - function of $S$.

Remarks. (a) An equivalent expression of $e$ follows from the identity

$$
\left(\operatorname{det}_{\mathfrak{s}} \operatorname{Ad} a(X, Y) b(X, Y)\right)^{-1}=\operatorname{det}_{\mathfrak{h}} \operatorname{Ad} a(X, Y) b(X, Y) .
$$

Indeed $\operatorname{det}_{\mathfrak{h}} \operatorname{Ad} a \operatorname{det}_{\mathfrak{s}} \operatorname{Ad} a=\operatorname{det}_{\mathfrak{g}} \operatorname{Ad} a$ for any $a \in H$, and

$$
\partial_{t} \log \operatorname{det}_{\mathfrak{g}} \operatorname{Ad} a_{t}(X, Y)=\operatorname{tr}_{\mathfrak{g}} \text { ad } A_{t}\left(\Phi_{t}(X, Y)\right)=0,
$$

where the first equality is proved as (4.27) below and the latter follows from $A_{t}(\ldots) \in[\mathfrak{s}, \mathfrak{s}]$. Thus $\operatorname{det}_{\mathfrak{g}} \operatorname{Ad} a_{t}(X, Y)=1$ which proves our claim.

Recall that, by Lemma 4.11, the $\operatorname{det}$ Ad factors in $e(X, Y)$ are identically 1 if $S$ has a $G$-semi-invariant measure.

(b) Let us emphasize that the $(j, F, G)$ - function is a special case of the $(j, \Phi)$ - function, corresponding to the specific choice of $A, B=A+C$ arising 
from (4.9) and (4.10). Section 4.4 will show the stronger properties of $e$ when $j=J^{1 / 2}$, the square root of the Jacobian of Exp.

(c) According to Theorem $4.6(v)$, the above is valid on $\Omega=\mathfrak{s} \times \mathfrak{s}$ if $H$ is compact and Exp : $\mathfrak{s} \rightarrow S$ is a global diffeomorphism.

(d) If $j$ is even, then $e(-X,-Y)=e(X, Y)$ because $a$ and $b$ are even by the proof of Theorem 4.6.

Proof of Theorem 4.12. For $(X, Y) \in \Omega$ we know from Theorem 4.6 that $\Phi(X, Y)$ belongs to $\mathcal{O}$ and $X+Y=Z(\Phi(X, Y))$ belongs to $\mathfrak{s}^{\prime}$, hence $j(X+Y)>0$ and the expression (4.23) of $e$ shows its analyticity on $\Omega$. It is $H$-invariant because of the invariance of $j, \Phi, a$ and $b$. No factor of $e$ vanishes, hence $e>0$ on $\Omega$ since $\Omega$ is connected and $e(0,0)=1$ (see (4.17) with $X=Y=0$ ). It will therefore be an $e$-function for $S$ if we prove that (Proposition 3.4)

$\left\langle u(X) \otimes v(Y), \frac{j(X) j(Y)}{j(Z(X, Y))} f(Z(X, Y))\right\rangle=\langle u(X) \otimes v(Y), e(X, Y) f(X+Y)\rangle$

This is done by deformation according to a parameter $t \in[0,1]$. Recalling $a_{t}, b_{t}$ and the diffeomorphisms $\Phi_{t}$ from (4.11)-(4.17), let $g \in C^{\infty}(\Omega)$ and $g_{t}$ be defined on $\Phi_{t}(\Omega)$ by

$$
\left(g_{t} \circ \Phi_{t}\right)(X, Y)=g(X, Y) \operatorname{det} D \Phi_{t}(X, Y)^{-1} \operatorname{det}_{\mathfrak{s}} \operatorname{Ad} a_{t}(X, Y) b_{t}(X, Y) .
$$

We shall prove that

$$
\left\langle u \otimes v, \partial_{t} g_{t}\right\rangle=0
$$

therefore

$$
\left\langle u \otimes v, g_{1}\right\rangle=\left\langle u \otimes v, g_{0}\right\rangle .
$$

Applying this to $g(X, Y):=e(X, Y) f(X+Y)$ with $e$ defined by (4.23), $f \in C^{\infty}(\mathfrak{s})$, and remembering the properties of $\Phi$ and the $H$-invariance of $j$ we obtain

$$
g_{1}(X, Y)=\frac{j(X) j(Y)}{j(Z(X, Y))} f(Z(X, Y)), g_{0}(X, Y)=e(X, Y) f(X+Y)
$$

and our claim will follow. The proof of (4.25) is split in the next two lemmas.

Lemma 4.14 With $A_{t}, B_{t}, \Phi_{t}$ as in (4.11)-(4.17) and $g_{t}$ defined by (4.24) we have

$$
\partial_{t} g_{t}=\operatorname{tr}_{\mathfrak{s}}\left(x \circ \partial_{X}\left(g_{t} A_{t}\right)+y \circ \partial_{Y}\left(g_{t} B_{t}\right)\right)
$$

on $\Phi_{t}(\Omega)$, with $x=\operatorname{ad} X, y=\operatorname{ad} Y$.

Proof. From (4.17) we know that

$\partial_{t} \log \operatorname{det}_{\mathfrak{s} \times \mathfrak{s}} D \Phi_{t}(X, Y)=\operatorname{tr}_{\mathfrak{s}}\left(\operatorname{ad}\left(A_{t}+B_{t}\right)-x \partial_{X} A_{t}-y \partial_{Y} B_{t}\right)\left(\Phi_{t}(X, Y)\right)$. 
Besides

$$
\begin{aligned}
\partial_{t} \log \operatorname{det}_{\mathfrak{s}} \operatorname{Ad} a_{t} & =\operatorname{tr}_{\mathfrak{s}}\left(\left(\operatorname{Ad} a_{t}^{-1} \circ D_{a_{t}} \operatorname{Ad}\right) \partial_{t} a_{t}\right) \\
& =\operatorname{tr}_{\mathfrak{s}} \operatorname{ad}\left(\left(D_{e} L_{a_{t}}\right)^{-1} \partial_{t} a_{t}\right)=\operatorname{tr}_{\mathfrak{s}} \operatorname{ad}\left(\operatorname{Ad} a_{t}^{-1}\left(A_{t} \circ \Phi_{t}\right)\right)
\end{aligned}
$$

by (4.11), therefore

$$
\begin{aligned}
& \partial_{t} \log \operatorname{det}_{\mathfrak{s}} \operatorname{Ad} a_{t}(X, Y)=\operatorname{tr}_{\mathfrak{s}} \operatorname{ad} A_{t}\left(\Phi_{t}(X, Y)\right) \\
& \partial_{t} \log \operatorname{det}_{\mathfrak{s}} \operatorname{Ad} b_{t}(X, Y)=\operatorname{tr}_{\mathfrak{s}} \operatorname{ad} B_{t}\left(\Phi_{t}(X, Y)\right),
\end{aligned}
$$

and the definition (4.24) implies

$$
\partial_{t}\left(g_{t} \circ \Phi_{t}\right)=\left(g_{t} \operatorname{tr}_{\mathfrak{s}}\left(x \partial_{X} A_{t}+y \partial_{Y} B_{t}\right)\right) \circ \Phi_{t} .
$$

On the other hand

$$
\partial_{t}\left(g_{t} \circ \Phi_{t}\right)=\left(\partial_{t} g_{t}+\left(\partial_{X} g_{t}\right)\left[A_{t}, X\right]+\left(\partial_{Y} g_{t}\right)\left[B_{t}, Y\right]\right) \circ \Phi_{t}
$$

by (4.13) and, putting together the latter equalities we obtain, after composition by $\Phi_{t}^{-1}$,

$$
\begin{aligned}
\partial_{t} g_{t} & =\left(\partial_{X} g_{t}\right) x A_{t}+\left(\partial_{Y} g_{t}\right) y B_{t}+\operatorname{tr}_{\mathfrak{s}}\left(g_{t} x \partial_{X} A_{t}+g_{t} y \partial_{Y} B_{t}\right) \\
& =\operatorname{tr}_{\mathfrak{s}}\left(x \partial_{X}\left(g_{t} A_{t}\right)+y \partial_{Y}\left(g_{t} B_{t}\right)\right) .
\end{aligned}
$$

Lemma 4.15 Let $u$ be a distribution on an open subset $U$ of $\mathfrak{s}$. If $u$ is $H$ invariant then, for any smooth map $F: U \rightarrow \mathfrak{h}$ such that $\operatorname{supp} u \cap \operatorname{supp} F$ is compact,

$$
\left\langle u(X), \operatorname{tr}_{\mathfrak{s}}\left(x \circ \partial_{X} F(X)\right)\right\rangle=0 .
$$

The converse holds true if $H$ is connected.

Proof. Repeat the proof of Lemma 1.4 with a basis $\left(E_{i}\right)$ of $\mathfrak{h}$.

Conversely, applying (4.28) to $F_{s}(X)=f\left(e^{-s \text { ad } E} X\right) E$ where $s \in \mathbb{R}$ and $E \in \mathfrak{h}$, we get

$$
\partial_{s}\left\langle u(X), f\left(e^{-s \operatorname{ad} E} X\right)\right\rangle=\left\langle u(X), \operatorname{tr}_{\mathfrak{s}}\left(x \circ \partial_{X} F_{s}(X)\right)\right\rangle=0
$$

and $u$ is $H$-invariant since $H$ is generated by all $\exp s E$.

Going back to the proof of Theorem 4.12 we apply Lemma 4.15 with $u$, $H, \mathfrak{s}, \mathfrak{h}, X$ and $F$ respectively replaced by $u \otimes v, H \times H, \mathfrak{s} \times \mathfrak{s}, \mathfrak{h} \times \mathfrak{h},(X, Y)$ and $\left(g_{t} A_{t}, g_{t} B_{t}\right)$ :

$$
\left\langle u(X) \otimes v(Y), \operatorname{tr}_{\mathfrak{s}}\left(x \circ \partial_{X}\left(g_{t} A_{t}\right)+y \circ \partial_{Y}\left(g_{t} B_{t}\right)\right)\right\rangle=0,
$$

and (4.25) follows in view of Lemma 4.14. 
As regards supports we need to make sure that, given $g \in C^{\infty}(\Omega),\left\langle u \otimes v, g_{t}\right\rangle$ is well defined for $0 \leq t \leq 1$ and Lemma 4.15 applies. But $\operatorname{supp} g_{t}=$ $\Phi_{t}\left(\operatorname{supp}\left(g_{t} \circ \Phi_{t}\right)\right)=\Phi_{t}(\operatorname{supp} g)$ in view of (4.24) and our proof works if $(\operatorname{supp} u \times \operatorname{supp} v) \cap \Phi_{t}(\operatorname{supp} g)$ is compact for all $t \in[0,1]$. In order to apply it to $g=e .\left.F\right|_{\Omega}$ with $F(X, Y):=f(X+Y)$, we assume $f \in C^{\infty}(\mathfrak{s})$ and $(\operatorname{supp} u \times \operatorname{supp} v) \cap \Phi_{t}(\Omega \cap \operatorname{supp} F)$ compact for all $t \in[0,1]$.

If $\operatorname{supp} v=\{0\}$ it is easily checked, remembering that $\Phi_{t}(X, 0)=(X, 0)$ for $(X, 0) \in \Omega$, that this assumption boils down to supp $u \cap \operatorname{supp} f \cap \Omega_{1}$ compact. This concludes the proof of Theorem 4.12.

\subsubsection{Application to Invariant Differential Operators}

An alteration of the proof of Theorem 4.12 leads to a more precise form of Theorem 3.8, extending to symmetric spaces Proposition 4.2 of [30] for Lie groups. We denote by $\xi_{V}$ the adjoint vector field associated to a vector $V \in \mathfrak{h}$, defined by

$$
\begin{aligned}
\xi_{V} f(X): & =\left.\partial_{s}\left(f\left(\operatorname{Ad}\left(e^{-s V}\right) X\right) \operatorname{det}_{\mathfrak{h}} \operatorname{Ad}\left(e^{s V}\right)\right)\right|_{s=0} \\
& =\langle D f(X),[X, V]\rangle+f(X) \operatorname{tr}_{\mathfrak{h}} \operatorname{ad} V
\end{aligned}
$$

where $f$ is smooth on an open subset $U$ of $\mathfrak{s}$ and $X \in U$.

Let $\mathcal{I}(U)$ be the left ideal generated by the $\xi_{V}$ 's with $V \in \mathfrak{h}_{*}=[\mathfrak{s}, \mathfrak{s}]$ in the algebra of all differential operators on $U$ with analytic coefficients. As before $H_{*}$ denotes the Lie subgroup of $H$ with Lie algebra $\mathfrak{h}_{*}$.

For $R \in \mathcal{I}(U)$ we have $R f=0$ whenever $f(X) d X$ is an $H_{*}$-invariant measure (in particular if it is $H$-invariant). Indeed this invariance is equivalent to $f(h \cdot X) \operatorname{det}_{\mathfrak{s}} \operatorname{Ad} h=f(X)$ for $h \in H_{*}, X \in U$. But for $V \in \mathfrak{h}_{*}$ we have $\operatorname{tr}_{\mathfrak{h}}$ ad $V+\operatorname{tr}_{\mathfrak{s}}$ ad $V=\operatorname{tr}_{\mathfrak{g}}$ ad $V=0$ and

$$
\operatorname{det}_{\mathfrak{h}} \operatorname{Ad}\left(e^{s V}\right)=\exp \left(s \operatorname{tr}_{\mathfrak{h}} \text { ad } V\right)=\exp \left(-s \operatorname{tr}_{\mathfrak{s}} \operatorname{ad} V\right)=\operatorname{det}_{\mathfrak{s}} \operatorname{Ad}\left(e^{-s V}\right)
$$

whence $\xi_{V} f=0$.

Furthermore, if $\operatorname{tr}_{\mathfrak{h}}$ ad $\mathfrak{h}_{*}=0$ (in particular if $G / H$ admits a $G$-invariant measure, see Lemma 4.11) we have $R f=0$ whenever $f$ is an $H_{*}$-invariant function (since $\operatorname{tr}_{\mathfrak{h}}$ ad $V=\operatorname{tr}_{\mathfrak{s}}$ ad $V=0$ in this case).

Theorem 4.16 Let $P=P(\partial) \in \mathbb{D}(\mathfrak{s})^{H}$ be a constant coefficients $H$-invariant differential operator on $\mathfrak{s}$, let $\widetilde{P} \in \mathbb{D}(S)^{G}$ be the corresponding $G$-invariant operator on $S$ (see 3.1.1) and let $P_{e}$ be defined as in Theorem 3.8 by

$$
P_{e}\left(X, \partial_{X}\right) f(X)=\left.P\left(\partial_{Y}\right)(e(X, Y) f(X+Y))\right|_{Y=0},
$$

where $e$ is the $(j, \Phi)$ - function (4.23).

Let $\Omega_{1}=\{X \in \mathfrak{s} \mid(X, 0) \in \Omega\}$. There exists a differential operator $R=$ $R\left(X, \partial_{X}\right) \in \mathcal{I}\left(\Omega_{1}\right)$ such that, for all $f \in C^{\infty}(\mathfrak{s})$,

$$
\widetilde{P} \widetilde{f}=\widetilde{P_{e} f}+\widetilde{R f} \text { on } \operatorname{Exp} \Omega_{1} \text {. }
$$


Proof. From the expression (3.3) of $\widetilde{P}$ and the definition of the map ${ }^{\sim}$ we have

$$
\begin{aligned}
\widetilde{P} \widetilde{f}(\operatorname{Exp} X) & =P\left(\partial_{Y}\right)\left(\left.j(Y) \widetilde{f}(\exp X \cdot \operatorname{Exp} Y)\right|_{Y=0}\right. \\
& =\left.P\left(\partial_{Y}\right)\left(\frac{j(Y)}{j(Z(X, Y))} f(Z(X, Y))\right)\right|_{Y=0},
\end{aligned}
$$

that is

$$
\widetilde{P} \widetilde{f}(\operatorname{Exp} X)=\widetilde{Q f}(\operatorname{Exp} X)
$$

where $Q=Q\left(X, \partial_{X}\right)$ is the differential operator with analytic coefficients defined by

$$
Q f(X):=\left.P\left(\partial_{Y}\right)\left(\frac{j(X) j(Y)}{j(Z(X, Y))} f(Z(X, Y))\right)\right|_{Y=0} .
$$

To prove the theorem we must show that the operator $R=Q-P_{e}$ belongs to $\mathcal{I}\left(\Omega_{1}\right)$. But, comparing $P_{e}$ and $Q$,

$$
R f(X)=\left.P\left(\partial_{Y}\right)\left(g_{1}(X, Y)-g_{0}(X, Y)\right)\right|_{Y=0}
$$

in the notation $g_{t}$ from (4.24) and (4.26).

We shall therefore study $P\left(\partial_{Y}\right)\left(\partial_{t} g_{t}(X, Y)\right)_{Y=0}$. Differentiation with respect to $Y$ will be easier from the following expression of $\partial_{t} g_{t}$, giving up the symmetry in Lemma 4.14. Let us recall the notation $Z_{t}(X, Y)=t^{-1} Z(t X, t Y)$.

\section{Lemma 4.17 Then}

$$
\partial_{t} g_{t}=\left(\xi_{V_{t}} f\right) \circ Z_{t}+\operatorname{tr}_{\mathfrak{s}}\left(y \partial_{Y}\left(g_{t} C_{t}\right)\right),
$$

where $C_{t}=B_{t}-A_{t}$ and $V_{t} \in \mathfrak{h}_{*}$ is defined at the end of the proof.

Proof. By Lemma 4.14

$$
\partial_{t} g_{t}=\operatorname{tr}_{\mathfrak{s}}\left(x \partial_{X}\left(g_{t} A_{t}\right)+y \partial_{Y}\left(g_{t} B_{t}\right)\right)=\operatorname{tr}_{\mathfrak{h}} u+\operatorname{tr}_{\mathfrak{s}}\left(y \partial_{Y}\left(g_{t} C_{t}\right)\right),
$$

where $u$ is the endomorphism of $\mathfrak{h}$ defined by $u:=\partial_{X}\left(g_{t} A_{t}\right) \circ x+\partial_{Y}\left(g_{t} A_{t}\right) \circ y$. For $W \in \mathfrak{h}$ we have

$$
u(W)=\left.\partial_{s}\left(\left(g_{t} A_{t}\right)\left(e^{-s W} \cdot X, e^{-s W} \cdot Y\right)\right)\right|_{s=0}
$$

and we must look at the behavior of $g_{t} A_{t}$ under the adjoint action of $e^{-s W} \in$ $H$. On the one hand $A_{t}(h \cdot X, h \cdot Y)=h \cdot A_{t}(X, Y)$ for any $h \in H$. On the other hand, remembering that $Z_{t} \circ \Phi_{t}(X, Y)=X+Y$, the definition (4.24) of $g_{t}$ may be rewritten as

$$
g_{t}(X, Y)=\gamma_{t}(X, Y) f\left(Z_{t}(X, Y)\right)
$$


where $\gamma_{t}$ is the function $g_{t}$ corresponding to $f=1$, that is

$$
\left(\gamma_{t} \circ \Phi_{t}\right)(X, Y)=e(X, Y) \operatorname{det} D \Phi_{t}(X, Y)^{-1} \operatorname{det}_{\mathfrak{s}} \operatorname{Ad} a_{t}(X, Y) b_{t}(X, Y) .
$$

But $\left(\gamma_{t} \circ \Phi_{t}\right)(h \cdot X, h \cdot Y)=\left(\gamma_{t} \circ \Phi_{t}\right)(X, Y)$ by the $H$-equivariance of $\Phi_{t}, a_{t}$, $b_{t}$ and $e$, therefore $\gamma_{t}(h \cdot X, h \cdot Y)=\gamma_{t}(X, Y)$. Then, looking back on $u$,

$$
u(W)=\left.\gamma_{t} \partial_{s}\left(f\left(e^{-s W} \cdot Z_{t}\right) e^{-s W} \cdot A_{t}\right)\right|_{s=0} .
$$

But for $V \in \mathfrak{h}$ and $X \in \mathfrak{s}$ we have

$$
\xi_{V} f(X)=\operatorname{tr}_{\mathfrak{h}}\left[\left.W \mapsto \partial_{s}\left(f\left(e^{-s W} \cdot X\right) e^{-s W} \cdot V\right)\right|_{s=0}\right]
$$

since this derivative at $s=0$ is $\langle D f(X),[X, W]\rangle V+f(X)$ ad $V(W)$. We finally obtain

$$
\operatorname{tr}_{\mathfrak{h}} u=\gamma_{t}\left(\xi_{A_{t}} f\right)\left(Z_{t}\right)=\left(\xi_{V_{t}} f\right)\left(Z_{t}\right)
$$

with $V_{t}:=\gamma_{t}(X, Y) A_{t}(X, Y) \in \mathfrak{h}_{*}$ and the lemma follows.

When applying $P\left(\partial_{Y}\right)$ at $Y=0$ to $\partial_{t} g_{t}$ as given by Lemma 4.17 , the second term vanishes in view of Lemma 4.15 for the $H$-invariant distribution ${ }^{t} P \delta_{0}$. Thus

$$
\left.P\left(\partial_{Y}\right)\left(\partial_{t} g_{t}(X, Y)\right)\right|_{Y=0}=\left.P\left(\partial_{Y}\right)\left(\xi_{V_{t}} f\right)\left(Z_{t}(X, Y)\right)\right|_{Y=0} .
$$

Let $\left(E_{i}\right)$ be a fixed basis of $\mathfrak{h}_{*}$ and $\xi_{i}=\xi_{E_{i}}$; decomposing $V_{t}$ according to this basis we obtain $\xi_{V_{t}}=\sum_{i} V_{i}(t, X, Y) \xi_{i}$ where the coefficients $V_{i}$ 's are analytic functions. Then, by Leibniz' formula in multi-index notation (with respect to some basis of $\mathfrak{s})$,

$$
\begin{aligned}
& \left.P\left(\partial_{Y}\right)\left(\partial_{t} g_{t}(X, Y)\right)\right|_{Y=0}= \\
& \quad=\sum_{i, \alpha} \frac{1}{\alpha !}\left(P^{(\alpha)}\left(\partial_{Y}\right) V_{i}\right)(t, X, 0) \partial_{Y}^{\alpha}\left(\left.\left(\xi_{i} f\right)\left(Z_{t}(X, Y)\right)\right|_{Y=0} .\right.
\end{aligned}
$$

But, $Y_{j}$ denoting the components of $Y$,

$$
\partial_{Y_{j}}\left(\left(\xi_{i} f\right)\left(Z_{t}(X, Y)\right)=\left\langle D\left(\xi_{i} f\right)\left(Z_{t}(X, Y), \partial_{Y_{j}} Z_{t}(X, Y)\right\rangle\right.\right.
$$

and, since $Z_{t}(X, 0)=X$, each $\partial_{Y}^{\alpha}\left(\left.\left(\xi_{i} f\right)\left(Z_{t}(X, Y)\right)\right|_{Y=0}\right.$ is obtained by applying to $\xi_{i} f$ a differential operator with analytic coefficients and evaluating at $X$. Finally

$$
\left.P\left(\partial_{Y}\right)\left(\partial_{t} g_{t}(X, Y)\right)\right|_{Y=0}=\sum_{i} R_{i}\left(t, X, \partial_{X}\right)\left(\xi_{i} f\right)(X)
$$

where the $R_{i}$ are differential operators on $\Omega_{1}$ with analytic coefficients. Integrating from 0 to 1 with respect to $t$ we obtain an equality of the form

$$
\left.P\left(\partial_{Y}\right)\left(g_{1}(X, Y)-g_{0}(X, Y)\right)\right|_{Y=0}=\sum_{i} R_{i}\left(X, \partial_{X}\right)\left(\xi_{i} f\right)(X)=R f(X)
$$

with $R=\sum_{i} R_{i}\left(X, \partial_{X}\right) \circ \xi_{i} \in \mathcal{I}\left(\Omega_{1}\right)$, as claimed. 


\subsection{Properties of Campbell-Hausdorff $e$-Functions}

We keep to the notation of Section 4.2 .

\subsection{1 $e$ and $c$}

We first give a new expression of $e$ by means of the element $c(X, Y)=$ $a(X, Y)^{-1} b(X, Y)$ of $H$ introduced in 4.2 .5 , similar to Corollary 3.17 in the Riemannian case ( $H$ compact).

Proposition 4.18 For $(X, Y) \in \Omega$ let $\psi_{X}(Y):=c(X, Y) \cdot Y$ and $\Psi(X, Y):=$ $\left(X, \psi_{X}(Y)\right)$. The map $\Psi$ is then a diffeomorphism of $\Omega$ onto $\Phi(\Omega)$ and

$$
\operatorname{det}_{\mathfrak{s} \times \mathfrak{s}} D \Phi(X, Y)=\operatorname{det}_{\mathfrak{s}} D \psi_{X}(Y)\left(\operatorname{det}_{\mathfrak{s}} \operatorname{Ad} a(X, Y)\right)^{3}
$$

(where $D \psi_{X}$ is the differential of $Y \mapsto \psi_{X}(Y)$ for fixed $\left.X\right)$. The $(j, \Phi)-$ function defined in Theorem 4.12 may be written as

$$
e(X, Y)=\frac{j(X) j(Y)}{j(X+Y)} \operatorname{det}_{\mathfrak{s}} D \psi_{X}(Y) \operatorname{det}_{\mathfrak{s}} \operatorname{Ad}\left(a(X, Y) c(X, Y)^{-1}\right) .
$$

Proof. Remembering the expression (4.23) of $e$ we only have to study $\Psi$ and prove (4.30). Let $h \cdot(X, Y):=(h \cdot X, h \cdot Y)$ denote the diagonal adjoint action of $h \in H$ on $\mathfrak{s} \times \mathfrak{s}$. The $H$-invariance $c(h \cdot(X, Y))=h c(X, Y) h^{-1}$ implies

$$
\Psi(h \cdot(X, Y))=(h \cdot X, c(h \cdot(X, Y)) h \cdot Y)=h \cdot \Psi(X, Y) .
$$

In particular, $h=a(X, Y)$ gives

$$
\Psi(a(X, Y) \cdot(X, Y))=(a(X, Y) \cdot X, b(X, Y) \cdot Y),
$$

that is

$$
\Psi \circ \Delta=\Phi \text { with } \Delta(X, Y):=a(X, Y) \cdot(X, Y) .
$$

Because $\Omega$ is $H$-invariant, $\Delta$ maps $\Omega$ into itself. Using $a(h \cdot(X, Y))=$ $h a(X, Y) h^{-1}$ with $h=a(X, Y)$, we obtain $(a \circ \Delta)(X, Y)=a(X, Y)$ and infer that $\Delta$ is a diffeomorphism of $\Omega$ onto itself, with $\Delta^{-1}(X, Y)=a(X, Y)^{-1}$. $(X, Y)$. Therefore $\Psi=\Phi \circ \Delta^{-1}$ is a diffeomorphism of $\Omega$ onto $\Phi(\Omega)$.

Besides $D \Phi(X, Y)=(D \Psi)(a(X, Y) \cdot(X, Y)) \circ D \Delta(X, Y)$ and the $H$-invariance of $\Psi$ gives $(D \Psi)(h \cdot(X, Y)) \circ \operatorname{Ad} h=\operatorname{Ad} h \circ D \Psi(X, Y)$ (where $\operatorname{Ad} h$ is the diagonal adjoint action) so that, with $h=a(X, Y)$ again,

$$
D \Phi(X, Y)=\operatorname{Ad} a(X, Y) \circ D \Psi(X, Y) \circ(\operatorname{Ad} a(X, Y))^{-1} \circ D \Delta(X, Y) .
$$

Since $\operatorname{det}_{\mathfrak{s} \times \mathfrak{s}} D \Psi(X, Y)=\operatorname{det}_{\mathfrak{s}} D \psi_{X}(Y)$ it will suffice to show that

$$
\operatorname{det}_{\mathfrak{s} \times \mathfrak{s}} D \Delta(X, Y)=\left(\operatorname{det}_{\mathfrak{s}} \operatorname{Ad} a(X, Y)\right)^{3} .
$$


Introducing a parameter $t$ let

$$
\Delta_{t}(X, Y):=t^{-1} \Delta(t X, t Y)=\left(a_{t}(X, Y) \cdot X, a_{t}(X, Y) \cdot Y\right)
$$

and $\Psi_{t}(X, Y):=t^{-1} \Psi(t X, t Y)$, so that $\Phi_{t}=\Psi_{t} \circ \Delta_{t}$. Let $\mathcal{A}_{t}:=A_{t} \circ \Psi_{t}$. By (4.11) we have

$$
\partial_{t} a_{t}=\left(D_{e} R_{a_{t}}\right)\left(A_{t} \circ \Phi_{t}\right)=\left(D_{e} R_{a_{t}}\right)\left(\mathcal{A}_{t} \circ \Delta_{t}\right)
$$

where all functions are evaluated at $(X, Y)$, therefore $\partial_{t} \log \operatorname{det}_{\mathfrak{s} \times \mathfrak{s}} D \Delta_{t}$ can be obtained as $\partial_{t} \log \operatorname{det}_{\mathfrak{s} \times \mathfrak{s}} D \Phi_{t}$ in $(4.17)$, with $b_{t}$ replaced by $a_{t}$ and $A_{t}$ by $\mathcal{A}_{t}$. Using (4.3) and Lemma 4.4 we infer that

$$
\begin{aligned}
\partial_{t} \log \operatorname{det}_{\mathfrak{s} \times \mathfrak{s}} D \Delta_{t} & =\operatorname{tr}_{\mathfrak{s}}\left(2 \operatorname{ad} \mathcal{A}_{t}-x \partial_{X} \mathcal{A}_{t}-y \partial_{Y} \mathcal{A}_{t}\right) \circ \Delta_{t} \\
& =\left(2 \operatorname{tr}_{\mathfrak{s}} \operatorname{ad} \mathcal{A}_{t}-\operatorname{tr}_{\mathfrak{h}} \operatorname{ad} \mathcal{A}_{t}\right) \circ \Delta_{t} \\
& =\left(2 \operatorname{tr}_{\mathfrak{s}} \operatorname{ad} A_{t}-\operatorname{tr}_{\mathfrak{h}} \operatorname{ad} A_{t}\right) \circ \Phi_{t} .
\end{aligned}
$$

But $\operatorname{tr}_{\mathfrak{h}}$ ad $A_{t}=-\operatorname{tr}_{\mathfrak{s}}$ ad $A_{t}$ since $A_{t} \in[\mathfrak{s}, \mathfrak{s}]$ implies $\operatorname{tr}_{\mathfrak{g}}$ ad $A_{t}=0$, whence $\partial_{t} \log \operatorname{det}_{\mathfrak{s} \times \mathfrak{s}} D \Delta_{t}=3 \operatorname{tr}_{\mathfrak{s}}$ ad $A_{t} \circ \Phi_{t}$ and our claim in view of (4.27).

\subsubsection{Some Deeper Properties of $e$}

Let $e_{t}(X, Y):=e(t X, t Y)$ with $e$ given by Theorem 4.12. By (4.15) and (4.16) we have

$$
e_{t}(X, Y)=\frac{j(t X) j(t Y)}{j(t X+t Y)} \operatorname{det}_{\mathfrak{s} \times \mathfrak{s}} D \Phi_{t}(X, Y)\left(\operatorname{det}_{\mathfrak{s}} \operatorname{Ad} a_{t}(X, Y) b_{t}(X, Y)\right)^{-1}
$$

All properties of $e$ in this subsection will follow from various expressions of the derivative $\partial_{t} \log e_{t}$ given by the next proposition. The particular choice $j(X)=J(X)^{1 / 2}=\left(\operatorname{det}_{\mathfrak{s}}(\operatorname{sh} x / x)\right)^{1 / 2}$ (the square root of the Jacobian of Exp) now becomes important.

For later reference let us recall the Kashiwara-Vergne equation (KV1) for the Lie algebra $\mathfrak{g}$ and the trace condition (KV2) (Section 1.5.1), with $(X, Y)$ replaced by $(2 X, 2 Y)$ for convenience:

$$
\begin{aligned}
& \frac{1}{2} \log \left(e^{2 Y} e^{2 X}\right)=X+Y-\left(1-e^{-2 x}\right) F(X, Y)-\left(e^{2 y}-1\right) G(X, Y) \\
& \frac{1}{4} \operatorname{tr}_{\mathfrak{g}}(x \operatorname{coth} x+y \operatorname{coth} y-v \operatorname{coth} v-1)=\operatorname{tr}_{\mathfrak{g}}\left(x \circ \partial_{X} F+y \circ \partial_{Y} G\right)
\end{aligned}
$$

Here $(X, Y)$ belongs to $\mathcal{U}$ (Section 4.2.2) and $v=\operatorname{ad} V$ with $V:=\frac{1}{2} \log e^{2 X} e^{2 Y}$. Any solution of (KV1) alone, or of (KV1) and (KV2), can be modified by Proposition 1.12 so as to satisfy $G(X, Y)=F(-Y,-X)$ too. The existence of Lie series $F, G$ solutions of (KV1) and (KV2) (the «Kashiwara-Vergne conjecture») has been proved in full generality (see Chapter 1). 
Proposition 4.19 (i) Let e be the $\left(J^{1 / 2}, \Phi\right)$ - function of a symmetric space and $e_{t}(X, Y)=e(t X, t Y)$. Then, for $(X, Y) \in \Omega$,

$$
\partial_{t} \log e_{t}(X, Y)=\mathcal{J}-\mathcal{F}
$$

with

$$
\begin{aligned}
\mathcal{J} & :=\frac{1}{2} \operatorname{tr}_{\mathfrak{h}}\left(x \operatorname{coth} t x+y \operatorname{coth} t y-(x+y) \operatorname{coth} t(x+y)-\frac{1}{t}\right) \\
& =\frac{1}{2} \operatorname{tr}_{\mathfrak{h}}\left(x \operatorname{coth} t x+y \operatorname{coth} t y-z \operatorname{coth} t z-\frac{1}{t}\right) \circ \Phi_{t}(X, Y),
\end{aligned}
$$

$z=\operatorname{ad} Z(X, Y)$, and

$$
\begin{aligned}
\mathcal{F} & :=\operatorname{tr}_{\mathfrak{h}}\left(\partial_{X} A_{t} \circ x+\partial_{Y} B_{t} \circ y\right) \circ \Phi_{t}(X, Y) \\
& =\operatorname{tr}_{\mathfrak{h}}\left(\partial_{Y} C_{t} \circ y+\operatorname{ad} A_{t}\right) \circ \Phi_{t}(X, Y) \\
& =\operatorname{tr}_{\mathfrak{h}}\left(-\partial_{X} C_{t} \circ x+\operatorname{ad} B_{t}\right) \circ \Phi_{t}(X, Y) .
\end{aligned}
$$

(ii) Lete be the $\left(J^{1 / 2}, F, G\right)$ - function, where $F$ and $G$ satisfy the KashiwaraVergne equations (KV1) and (KV2). Then, for $(X, Y) \in \Omega$,

$$
\begin{aligned}
\partial_{t} \log e_{t}(X, Y) & =\operatorname{tr}_{\mathfrak{h}}\left(\frac{1}{2}\left[y, \partial_{Y} C_{t}\right]-\operatorname{ad} A_{t}\right) \circ \Phi_{t}(X, Y) \\
& =\operatorname{tr}_{\mathfrak{h}}\left(\frac{1}{2}\left[\partial_{X} C_{t}, x\right]-\operatorname{ad} B_{t}\right) \circ \Phi_{t}(X, Y) .
\end{aligned}
$$

Also, with $U_{t}=c_{t}(X, Y) \cdot Y$ given by Lemma 4.7,

$$
\partial_{t} \log e_{t}(X, Y)=\operatorname{tr}_{\mathfrak{h}}\left(\frac{1}{2}\left[\left(\partial_{X} C_{t}\right)\left(X, U_{t}\right), x\right]-\operatorname{ad} B_{t}\left(X, U_{t}\right)\right) .
$$

Remarks. (a) The ad $A_{t}$ and ad $B_{t}$ terms in these equalities can be forgotten if $\operatorname{tr}_{\mathfrak{h}}$ ad $\mathfrak{h}_{*}=0$, for example when the symmetric space $G / H$ has a $G$-invariant measure (Lemma 4.11). The $\left(J^{1 / 2}, F, G\right)$ - function can then be written in terms of $C$ only, with $U_{t}$ given by (4.20). See also Proposition 4.18 above, applying to the more general $(j, \Phi)$ - function.

(b) Writing $\partial_{t} \log e_{t}$ by means of (4.33) and (4.34) shows that it embodies the obstruction of an analog of (KV2) for symmetric spaces.

Proof. In order to lighten notation we compute derivatives at $t=1$ only. The general case will follow from the behavior of all terms under scalings $(X, Y) \mapsto(t X, t Y)$ (see (4.16)).

(i) The terms in $\mathcal{J}$ come from the $J^{1 / 2}$ factors in $e_{t}$, those in $\mathcal{F}$ from $\Phi_{t}$, $a_{t}$ and $b_{t}$. The definition $J(X)=\operatorname{det}_{\mathfrak{s}}(\operatorname{sh} x / x)$ implies $\left.\partial_{t} \log J(t X)\right|_{t=1}=$ $\operatorname{tr}_{\mathfrak{s}}(x \operatorname{coth} x-1)$, therefore

$$
\begin{aligned}
\partial_{t} \log \left(\frac{J(t X) J(t Y)}{J(t X+t Y)}\right)_{t=1}^{1 / 2} & = \\
= & \frac{1}{2} \operatorname{tr}_{\mathfrak{s}}(x \operatorname{coth} x+y \operatorname{coth} y-(x+y) \operatorname{coth}(x+y)-1) .
\end{aligned}
$$


We may replace here $\operatorname{tr}_{\mathfrak{s}}$ by $\operatorname{tr}_{\mathfrak{h}}$ because of (4.3). Since $X+Y=Z \circ \Phi(X, Y)$ and $\Phi(X, Y)=(a \cdot X, b \cdot Y)$ by Theorem 4.6, this may be rewritten as

$\partial_{t} \log \left(\frac{J(t X) J(t Y)}{J(t X+t Y)}\right)_{t=1}^{1 / 2}=\frac{1}{2} \operatorname{tr}_{\mathfrak{h}}(x \operatorname{coth} x+y \operatorname{coth} y-z \operatorname{coth} z-1) \circ \Phi(X, Y)$

with $z=\operatorname{ad} Z(X, Y)$, and both formulas for $\mathcal{J}$ are proved.

Combining this with the derivatives of the determinants in $e_{t}$ (see the proof of Lemma 4.14) we obtain

$$
\left.\partial_{t} \log e_{t}\right|_{t=1}=\mathcal{J}-\mathcal{F}, \text { with } \mathcal{F}=\operatorname{tr}_{\mathfrak{s}}\left(x \partial_{X} A+y \partial_{Y} B\right) \circ \Phi .
$$

In view of Lemma $4.4(b)$ the latter term can be changed into

$$
\begin{aligned}
\mathcal{F} & =\operatorname{tr}_{\mathfrak{h}}\left(\partial_{X} A \circ x+\partial_{Y} B \circ y\right) \circ \Phi=\operatorname{tr}_{\mathfrak{h}}\left(\partial_{Y} C \circ y+\operatorname{ad} A\right) \circ \Phi \\
& =\operatorname{tr}_{\mathfrak{h}}\left(-\partial_{X} C \circ x+\operatorname{ad} B\right) \circ \Phi
\end{aligned}
$$

with $C=B-A$. This proves (4.34), (4.35) and (4.36).

(ii) Let us now compare with the Kashiwara-Vergne trace condition (KV2). Since $Z(X, Y)=e^{-x} V$ by Proposition 4.2, $v$ can be replaced by $z=e^{-x} v e^{x}$ in the left-hand side. Using Lemma $4.4(a)$ to modify the right-hand side we see that (KV2) is equivalent to

$$
\frac{1}{4} \operatorname{tr}_{\mathfrak{g}}(x \operatorname{coth} x+y \operatorname{coth} y-z \operatorname{coth} z-1)=\operatorname{tr}_{\mathfrak{g}}\left(\partial_{Y}(G-F) \circ y+\operatorname{ad} F\right) .
$$

For the symmetric space we have chosen $A=e^{-x} F+e^{x} F^{\vee}$ and $C=(G-$ $F)+(G-F)^{\vee}$ in $(4.9),(4.10)$ with $F^{\vee}(X, Y)=F(-X,-Y)$ etc. Adding (4.40) and $(4.40)^{\vee},(\mathrm{KV} 2)$ implies

$$
\frac{1}{2} \operatorname{tr}_{\mathfrak{g}}(x \operatorname{coth} x+y \operatorname{coth} y-z \operatorname{coth} z-1)=\operatorname{tr}_{\mathfrak{g}}\left(\partial_{Y} C \circ y\right) ;
$$

indeed $Z^{\vee}=-Z$ by Proposition 4.2 and $\operatorname{tr}_{\mathfrak{g}}$ ad $\left(F+F^{\vee}\right)=0$ because $F+F^{\vee}$ is an even series of Lie brackets, thus maps $\mathcal{U}$ into $[\mathfrak{g}, \mathfrak{g}]$.

Restricting now to $(X, Y) \in \mathcal{O} \subset \mathfrak{s} \times \mathfrak{s}$ we may replace, in view of (4.2) and (4.3), $\operatorname{tr}_{\mathfrak{g}}$ by $\operatorname{tr}_{\mathfrak{h}}+\operatorname{tr}_{\mathfrak{s}}=2 \operatorname{tr}_{\mathfrak{h}}$ in the left-hand side and by $\operatorname{tr}_{\mathfrak{h}}+\operatorname{tr}_{\mathfrak{s}}$ in the right-hand side. Thus, for $(X, Y) \in \mathcal{O}$,

$$
\frac{1}{2} \operatorname{tr}_{\mathfrak{h}}(x \operatorname{coth} x+y \operatorname{coth} y-z \operatorname{coth} z-1)=\frac{1}{2}\left(\operatorname{tr}_{\mathfrak{h}}+\operatorname{tr}_{\mathfrak{s}}\right)\left(\partial_{Y} C \circ y\right) .
$$

Together with (4.33) and (4.35) we obtain

$$
\left.\partial_{t} \log e_{t}\right|_{t=1}=\left(\frac{1}{2}\left(\operatorname{tr}_{\mathfrak{s}}-\operatorname{tr}_{\mathfrak{h}}\right)\left(\partial_{Y} C \circ y\right)-\operatorname{tr}_{\mathfrak{h}} \operatorname{ad} A\right) \circ \Phi
$$

on $\Omega$, and finally (4.37) because $\operatorname{tr}_{\mathfrak{s}}\left(\partial_{Y} C \circ y\right)=\operatorname{tr}_{\mathfrak{h}}\left(y \circ \partial_{Y} C\right)$. A similar proof gives (4.38) - or else (4.38) is equivalent to (4.37) in view of Lemma 4.4 and $\operatorname{tr}_{\mathfrak{g}}$ ad $C=0$. 
With $c=a^{-1} b$ we have $\Phi(X, Y)=(a \cdot X, b \cdot Y)=a \cdot(X, c \cdot Y)$ (diagonal adjoint action of $a \in H$ on $\mathfrak{s} \times \mathfrak{s})$, thus

$$
\operatorname{ad}(B \circ \Phi)(X, Y)=\operatorname{Ad} a \circ \operatorname{ad}(B(X, c \cdot Y)) \circ \operatorname{Ad} a^{-1}
$$

and similarly for $\left[\partial_{X} C_{t}, x\right]$. Consequently (4.39) follows from (4.38).

Theorem 4.20 (structure of $e$-functions) If Lie series F, G satisfy (KV1) and (KV2) the corresponding $\left(J^{1 / 2}, F, G\right)$ - function has the following properties: there exist sequences $\left(a_{2 p}\right),\left(b_{2 p}\right),\left(c_{2 p}\right),\left(v_{2 p-1}\right),\left(w_{2 p-1}\right)$, of noncommutative homogeneous polynomials in $x=\operatorname{ad} X$ and $y=\operatorname{ad} Y$ (with degrees given by the subscripts) such that, in a neighborhood of the origin of $\mathfrak{s} \times \mathfrak{s}$,

(i) $\log e(X, Y)=\sum_{1}^{\infty} \operatorname{tr}_{\mathfrak{h}}\left(\left[x, v_{2 p-1}\right]+\left[y, w_{2 p-1}\right]\right)=\sum_{1}^{\infty}\left(\operatorname{tr}_{\mathfrak{s}}-\operatorname{tr}_{\mathfrak{h}}\right) a_{2 p}=$ $\sum_{1}^{\infty}\left(\operatorname{tr}_{\mathfrak{g}}-2 \operatorname{tr}_{\mathfrak{h}}\right) a_{2 p}$

(ii) $\log e(X, Y)=\sum_{0}^{\infty} \operatorname{tr}_{\mathfrak{h}}\left([x, y] b_{2 p}\right)+\sum_{0}^{\infty} \operatorname{tr}_{\mathfrak{s}}\left([x, y] c_{2 p}\right)$.

In particular $e(X, Y)=1$ for any $(X, Y) \in \Omega$ such that $[X, Y]=0$.

Remarks. (a) All series above are absolutely convergent in a neighborhood of the origin.

(b) Property (i) proves a conjecture of [44] p. 255.

(c) There exists a sequence $\left(f_{p}\right)$ of homogeneous polynomials in four (noncommutative) variables such that $\log e(X, Y)=\sum_{1}^{\infty} \operatorname{tr}_{\mathfrak{s}} f_{p}\left(x^{2}, x y, y x, y^{2}\right)$. Indeed $\log e(X, Y)=\sum_{1}^{\infty} \operatorname{tr}_{\mathfrak{s}} u_{2 p}$ by (i) with $u_{2 p}=\left[v_{2 p-1}, x\right]+\left[w_{2 p-1}, y\right]$, and an easy induction on the degree shows that each monomial in the even polynomial $u_{2 p}$ may be written as a monomial in $x^{2}, x y, y x$ and $y^{2}$.

Since $x y$ is the endomorphism $V \mapsto[V, Y, X]$ of the Lie triple system $\mathfrak{s}$ (Section 0.3 ) and similarly for $x^{2}, y x, y^{2}$, we see that $e$ is directly linked to the structure of Lie triple system corresponding to our symmetric space $S$. When replacing $S$ by the contracted space $S_{t}$ (Section 4.1), $x y$ etc. is multiplied by $t^{2}$ therefore $e_{t}(X, Y)=e(t X, t Y)$ is an $e$-function for $S_{t}$. When replacing $S$ by the dual space $S_{*}, x y$ etc. changes into $-x y$ etc. therefore $e_{*}(X, Y)=e(i X, i Y)$ is an $e$-function for $S_{*}$.

Besides the $f_{p}$ 's are obtained from $(F, G)$ in (KV1), therefore universal for all symmetric spaces if $(F, G)$ are universal.

(d) From (ii) we infer that there exists a sequence $\left(\lambda_{p}\right)$ of scalars such that

$$
\log e(X, Y)=\sum_{0}^{\infty} \lambda_{p} \operatorname{tr}_{\mathfrak{h}} \text { ad }\left(x^{2 p+1} Y\right)+O\left(Y^{2}\right),
$$

whence $\partial_{Y} e(X, 0)=0$ if $\operatorname{tr}_{\mathfrak{h}}$ ad $\mathfrak{h}_{*}=0$; Proposition 4.36 will provide a more precise result. Indeed $\log e(X, Y)$ is, modulo $Y^{2}$, a sum of terms of the form (with scalars $\beta_{p}, \gamma_{p}$ ):

$$
\begin{aligned}
\operatorname{tr}_{\mathfrak{h}}\left([x, y] \beta_{p} x^{2 p}\right)+\operatorname{tr}_{\mathfrak{s}}\left([x, y] \gamma_{p} x^{2 p}\right) & =\left(\beta_{p}-\gamma_{p}\right) \operatorname{tr}_{\mathfrak{h}}\left[x^{2 p+1}, y\right] \\
& =2^{-2 p}\left(\beta_{p}-\gamma_{p}\right) \operatorname{tr}_{\mathfrak{h}} \text { ad }\left(x^{2 p+1} Y\right)
\end{aligned}
$$


by Lemma 4.37 .

(e) The equality $e(X, Y)=1$ is actually valid under a weaker assumption: see Theorem $4.22(i)$ below.

Proof. (i) Since $e$ is analytic, even, and $e(0,0)=1$ we know that

$$
\log e(t X, t Y)=\sum_{p \geq 1} t^{2 p} e_{2 p}(X, Y),
$$

where $e_{2 p}$ is homogeneous of degree $2 p$ in $(X, Y)$, and there exists $R>0$ such that the series converges absolutely for $\|X\|<R,\|Y\|<R$ and $|t| \leq 1$. We shall obtain the coefficients $e_{2 p}$ from (4.39) by identification of formal power series with respect to $t$.

Let us consider first the solution $U_{t}=c(t X, t Y) \cdot Y$ of the differential equation $\partial_{t} U_{t}=\left[C_{t}\left(X, U_{t}\right), U_{t}\right], U_{0}=Y$ (Lemma 4.7). On the right-hand side is a Lie series of the form

$$
\left[C_{t}(X, Y), Y\right]=\sum_{q \geq 1} t^{2 q-1} \sum_{|\alpha|+|\beta|=2 q+1} c_{\alpha \beta} Z_{\alpha \beta}(X, Y)
$$

where the $c_{\alpha \beta}$ 's are coefficients and $Z_{\alpha \beta}(X, Y)=x^{\alpha_{1}} y^{\beta_{1}} \cdots x^{\alpha_{n}} Y$. We claim that $U_{t}$ can be expanded as ${ }^{3}$

$$
U_{t}=Y+\sum_{p \geq 1} t^{2 p} U_{2 p+1}
$$

where $U_{2 p+1}$ is a homogeneous Lie polynomial in $(X, Y)$ of degree $2 p+1$. Indeed, substituting $U_{t}$ for $Y$,

$$
Z_{\alpha \beta}\left(X, U_{t}\right)=Z_{\alpha \beta}(X, Y)+\sum_{r \geq 1} t^{2 r} Z_{\alpha \beta}^{r}\left(X, Y, U_{3}, \ldots, U_{2 r+1}\right)
$$

where $Z_{\alpha \beta}^{r}$ is a homogeneous Lie polynomial in $\left(X, \ldots, U_{2 r+1}\right)$. The differential equation for $U_{t}$ becomes

$$
\begin{aligned}
\sum_{p \geq 1} 2 p t^{2 p-1} U_{2 p+1} & =\sum_{q \geq 1} t^{2 q-1} \times \\
\times & \sum_{|\alpha|+|\beta|=2 q+1} c_{\alpha \beta}\left(Z_{\alpha \beta}(X, Y)+\sum_{r \geq 1} t^{2 r} Z_{\alpha \beta}^{r}\left(X, Y, \ldots, U_{2 r+1}\right)\right)
\end{aligned}
$$

and determines the $U_{2 p+1}$ 's inductively, Lie polynomials in $(X, Y)$ of degree $2 p+1$.

Similarly $B_{t}(X, Y)=\sum_{p \geq 1} t^{2 p-1} \sum_{|\alpha|+|\beta|=2 p} b_{\alpha \beta} Z_{\alpha \beta}(X, Y)$. Substituting $U_{t}$ for $Y$ and reordering according to the powers of $t$ we obtain $B_{t}\left(X, U_{t}\right)=$

\footnotetext{
${ }^{3}$ An explicit expression of $U_{3}$ and $U_{5}$ is given by Lemma 4.30 .
} 
$\sum_{p>1} t^{2 p-1} B_{2 p}(X, Y)$, where $B_{2 p}$ is a homogeneous Lie polynomial in $(X, Y)$ of degree $2 p$. Each $B_{2 p}$ begins with $[X, \ldots]$ or $[Y, \ldots]$ and, separating both types, it follows that

$$
\text { ad } B_{t}\left(X, U_{t}\right)=\sum_{p \geq 1} t^{2 p-1}\left(\left[x, b_{2 p-1}^{\prime}(x, y)\right]+\left[y, b_{2 p-1}^{\prime \prime}(x, y)\right]\right)
$$

with $b_{2 p-1}^{\prime}, b_{2 p-1}^{\prime \prime}$ homogeneous non-commutative polynomials in $(x, y)$ of degree $2 p-1$.

Next $\partial_{X} C_{t}(X, Y)=\sum_{p \geq 1} t^{2 p-1} c_{2 p-1}(x, y)$ with $c_{2 p-1}$ of degree $2 p-1$ and, replacing $y$ by ad $U_{t}$ and reordering,

$$
\left(\partial_{X} C_{t}\right)\left(X, U_{t}\right)=\sum_{p \geq 1} t^{2 p-1} c_{2 p-1}^{\prime}(x, y) .
$$

Finally (4.39) becomes, in similar notation,

$$
\begin{aligned}
\partial_{t} \log e(t X, t Y) & =\operatorname{tr}_{\mathfrak{h}}\left(\frac{1}{2}\left[\left(\partial_{X} C_{t}\right)\left(X, U_{t}\right), x\right]-\operatorname{ad} B_{t}\left(X, U_{t}\right)\right) \\
& =\sum_{p \geq 1} t^{2 p-1} \operatorname{tr}_{\mathfrak{h}}\left(\left[x, f_{2 p-1}(x, y)\right]+\left[y, g_{2 p-1}(x, y)\right]\right),
\end{aligned}
$$

whence the first claim in (i) with

$$
v_{2 p-1}=\frac{1}{2 p} f_{2 p-1}(x, y), w_{2 p-1}=\frac{1}{2 p} g_{2 p-1}(x, y) .
$$

Then $\operatorname{tr}_{\mathfrak{h}}\left[x, v_{2 p-1}\right]=\operatorname{tr}_{\mathfrak{h}}\left(x v_{2 p-1}-v_{2 p-1} x\right)=\left(\operatorname{tr}_{\mathfrak{h}}-\operatorname{tr}_{\mathfrak{s}}\right)\left(x v_{2 p-1}\right)$ by $(4.3)$, hence the second expression of $\log e$ with $a_{2 p}=x v_{2 p-1}+y w_{2 p-1}$, even. The third follows since $\operatorname{tr}_{\mathfrak{g}} a_{2 p}=\operatorname{tr}_{\mathfrak{h}} a_{2 p}+\operatorname{tr}_{\mathfrak{s}} a_{2 p}$.

(ii) The bracket $[x, \ldots]$ acts as a derivation on all monomials $x^{\alpha_{1}} y^{\beta_{1}} \cdots x^{\alpha_{k}} y^{\beta_{k}}$ in $v_{2 p-1}$, giving

$$
\left[x, v_{2 p-1}\right]=\sum_{j} b_{j}[x, y] b_{j}^{\prime}+\sum_{k} c_{k}[x, y] c_{k}^{\prime}
$$

with $b_{j}, b_{j}^{\prime}$ even and $c_{k}, c_{k}^{\prime}$ odd. Thus

$$
\operatorname{tr}_{\mathfrak{h}}\left[x, v_{2 p-1}\right]=\operatorname{tr}_{\mathfrak{h}}\left([x, y] \sum b_{j}^{\prime} b_{j}\right)+\operatorname{tr}_{\mathfrak{s}}\left([x, y] \sum c_{k}^{\prime} c_{k}\right),
$$

the same for $\operatorname{tr}_{\mathfrak{h}}\left[y, w_{2 p-1}\right]$, and (i) implies (ii).

If $[X, Y]=0$ then $[x, y]=\operatorname{ad}[X, Y]=0$ and (ii) applies to $(t X, t Y)$ for $|t|$ small enough, giving $\log e(t X, t Y)=0$. For $(X, Y) \in \Omega$ the function $t \mapsto \log e(t X, t Y)$ is analytic on an open interval containing [-1,1], therefore $\log e(X, Y)=0$ by analytic continuation.

Definition 4.21 A symmetric Lie algebra $(\mathfrak{g}, \sigma)$ is called strongly symmetric if there exists a linear isomorphism $\gamma$ of $\mathfrak{g}$ which commutes with all ad $X$ for $X \in \mathfrak{g}$ and anticommutes with $\sigma$. A symmetric space with strongly symmetric Lie algebra is called strongly symmetric. 
In other words $\gamma$ maps $\mathfrak{h}$ onto $\mathfrak{s}, \mathfrak{s}$ onto $\mathfrak{h}$ and $\gamma \circ \operatorname{ad} X=\operatorname{ad} X \circ \gamma=\operatorname{ad}(\gamma(X))$ for all $X \in \mathfrak{g}$; the latter equality follows from $\operatorname{ad}(\gamma(X)) Y=-(\operatorname{ad} Y \circ \gamma) X=$ $-(\gamma \circ \operatorname{ad} Y) X=(\gamma \circ$ ad $X) Y$. This property implies $\operatorname{dim} \mathfrak{g}=2 \operatorname{dim} \mathfrak{h}=$ $2 \operatorname{dim} \mathfrak{s}$. The basic examples of strongly symmetric Lie algebras are:

(a) $\left(\mathfrak{g}_{\mathbb{C}}, \sigma\right)$ where the symmetry $\sigma$ is the conjugation with respect to a real form $\mathfrak{h}=\mathfrak{g}_{\mathbb{R}}$ of the complex Lie algebra $\mathfrak{g}_{\mathbb{C}}$ and $\gamma$ is the multiplication by $i$ (b) $(\mathfrak{g} \times \mathfrak{g}, \sigma)$ where $\mathfrak{g}$ is a Lie algebra, $\sigma\left(X_{1}, X_{2}\right)=\left(X_{2}, X_{1}\right), \mathfrak{h}$ is the diagonal subalgebra of $\mathfrak{g} \times \mathfrak{g}$ and $\gamma\left(X_{1}, X_{2}\right)=\left(X_{1},-X_{2}\right)$.

These examples are dual to each other: the map $X+i Y \mapsto(X, X)+i(Y,-Y)$ identifies (a) with the dual of the symmetric Lie algebra (b) ([31] p. 253). The corresponding strongly symmetric spaces are $G_{\mathbb{C}} / G_{\mathbb{R}}$ (a complex Lie group modulo a real form) and a Lie group viewed as a symmetric space.

Let us recall that a symmetric space is called special if 1 is an $e$-function. Properties of special spaces are studied in Section 3.2.

Theorem 4.22 (special symmetric spaces) For a symmetric space with the $\left(J^{1 / 2}, F, G\right)$ - function constructed from $j=J^{1 / 2}$ and Lie series $F, G$ satisfying the Kashiwara-Vergne equations (KV1) and (KV2), the following holds.

(i) Let $(X, Y) \in \Omega$ such that $X$ and $Y$ belong to a solvable Lie subalgebra of $\mathfrak{g}$. Then $e(X, Y)=1$. In particular, solvable symmetric spaces are special.

(ii) Strongly symmetric spaces are special.

(iii) $e$ is identically 1 on $\Omega$ if and only if $\operatorname{tr}_{\mathfrak{h}} \operatorname{ad}_{\mathfrak{h}}=0$ and $\operatorname{tr}_{\mathfrak{h}}\left[y, \partial_{Y} C\right]=0$ for all $(X, Y) \in \mathcal{O}$. Then $\operatorname{det}_{\mathfrak{s} \times \mathfrak{s}} D \Phi(X, Y)=(J(X+Y) / J(X) J(Y))^{1 / 2}$.

Proof. Replacing $(X, Y)$ by $(t X, t Y)$ if necessary, we work in a neighorhood of the origin where Theorem 4.20 applies. The result will extend by analytic continuation on $t$.

(i) Let $\mathfrak{g}^{\prime}$ be a solvable subalgebra of $\mathfrak{g}$ containing $X$ and $Y$. By Lie's theorem for the adjoint representation of $\mathfrak{g}^{\prime}$ on the complexification $\mathfrak{g}_{\mathbb{C}}$ of $\mathfrak{g}$, there exists a basis of $\mathfrak{g}_{\mathbb{C}}$ in which $x=\operatorname{ad} X$ and $y=\operatorname{ad} Y$ are given by upper triangular matrices. The same holds for $v_{2 p-1}$ and $w_{2 p-1}$ in Theorem $4.20(i)$ therefore $\left[x, v_{2 p-1}\right]$ and $\left[y, w_{2 p-1}\right]$ are nilpotent, their $\operatorname{tr}_{\mathfrak{h}}$ vanishes and $\log e(X, Y)=0$. (ii) Now assume the space is strongly symmetric. Given $X, Y \in \mathfrak{s}$ let $v$ be an odd (non-commutative) monomial in $x=\operatorname{ad} X$ and $y=\operatorname{ad} Y$ of degree $2 p-1 \geq 1$. Let us write $X=\gamma\left(X^{\prime}\right), Y=\gamma\left(Y^{\prime}\right)$ with $X^{\prime}, Y^{\prime} \in \mathfrak{h}$, and let $v^{\prime}$ denote the monomial obtained from $v$ by replacing $x$ by $x^{\prime}=\operatorname{ad} X^{\prime}$ and $y$ by $y^{\prime}=\operatorname{ad} Y^{\prime}$. Observing that $x=\gamma \circ x^{\prime}=x^{\prime} \circ \gamma, y=\gamma \circ y^{\prime}=y^{\prime} \circ \gamma$ and that $x^{\prime}, v^{\prime}, \gamma^{2}$ are endomorphisms of $\mathfrak{h}$, we have

$$
\operatorname{tr}_{\mathfrak{h}}(x v)=\operatorname{tr}_{\mathfrak{h}}\left(x^{\prime} \gamma^{2 p} v^{\prime}\right)=\operatorname{tr}_{\mathfrak{h}}\left(\gamma^{2 p} v^{\prime} x^{\prime}\right)=\operatorname{tr}_{\mathfrak{h}}(v x),
$$

therefore $\operatorname{tr}_{\mathfrak{h}}[x, v]=0$ for any odd monomial $v$ in $x$ and $y$. Similarly $\operatorname{tr}_{\mathfrak{h}}[y, w]=$ 0 for $w$ odd, hence $\log e=0$ by $^{4}$ Theorem $4.20(i)$.

\footnotetext{
${ }^{4}$ The result also follows from (4.37) directly, since we have here $\operatorname{tr}$ ad $*=0$ and $\operatorname{tr}\left[y, \partial_{Y} C\right]=0, C$ being even.
} 
(iii) If $\operatorname{tr}_{\mathfrak{h}}\left[y, \partial_{Y} C\right]=0$ and $\operatorname{tr}_{\mathfrak{h}}$ ad $\mathfrak{h}_{*}=0$, then (4.37) implies $\partial_{t} \log e_{t}=0$ and $e$ is identically 1 . Conversely, the expansion $\log e(X, Y)=\frac{1}{4} \operatorname{tr}_{\mathfrak{h}} \operatorname{ad}[X, Y]+$ $O_{4}$ (Proposition 4.31) shows that $e \equiv 1$ implies $\operatorname{tr}_{\mathfrak{h}}$ ad $\mathfrak{h}_{*}=0$, therefore $\operatorname{tr}_{\mathfrak{h}}$ ad $A=0$ and finally $\operatorname{tr}_{\mathfrak{h}}\left[y, \partial_{Y} C\right]=0$ by (4.37). The latter result, valid in a neighborhood of the origin in $\mathfrak{s} \times \mathfrak{s}$, extends to $\mathcal{O}$ by analytic continuation. The expression of $\operatorname{det} D \Phi$ follows from (4.23) and Lemma 4.11.

Remarks. (a) Let $B_{\mathfrak{g}}$, resp. $B_{\mathfrak{h}}$, denote the Killing forms of the Lie algebras $\mathfrak{g}$, resp. $\mathfrak{h}$. Considering the second term in the expansion of $\log e$ in Proposition 4.31 we see that $e \equiv 1$ on $\Omega$ also implies $B_{\mathfrak{g}}-2 B_{\mathfrak{h}}=0$ on $\mathfrak{h}_{*} \times \mathfrak{h}_{*}$. (b) The above proof of (ii) shows that, for all strongly symmetric Lie algebras, $\operatorname{tr}_{\mathfrak{h}}[x, y]=0$ for $X, Y \in \mathfrak{s}$, that is $\operatorname{tr}_{\mathfrak{h}}$ ad $\mathfrak{h}_{*}=0$. Besides, for $X^{\prime}, Y^{\prime} \in \mathfrak{h}$ we have

$$
\left(B_{\mathfrak{g}}-2 B_{\mathfrak{h}}\right)\left(X^{\prime}, Y^{\prime}\right)=\left(\operatorname{tr}_{\mathfrak{h}}+\operatorname{tr}_{\mathfrak{s}}\right)\left(x^{\prime} y^{\prime}\right)-2 \operatorname{tr}_{\mathfrak{h}}\left(x^{\prime} y^{\prime}\right)=\left(\operatorname{tr}_{\mathfrak{s}}-\operatorname{tr}_{\mathfrak{h}}\right)\left(x^{\prime} y^{\prime}\right) .
$$

But $X^{\prime}=\gamma(X), Y^{\prime}=\gamma(Y)$ for some $X, Y \in \mathfrak{s}$, thus $x^{\prime}=\gamma x=x \gamma, y^{\prime}=\gamma y=$ $y \gamma$ and

$$
\operatorname{tr}_{\mathfrak{s}}\left(x^{\prime} y^{\prime}\right)=\operatorname{tr}_{\mathfrak{s}}(\gamma x \gamma y)=\operatorname{tr}_{\mathfrak{h}}(y \gamma x \gamma)=\operatorname{tr}_{\mathfrak{h}}\left(y^{\prime} x^{\prime}\right)=\operatorname{tr}_{\mathfrak{h}}\left(x^{\prime} y^{\prime}\right) .
$$

Therefore $B_{\mathfrak{g}}-2 B_{\mathfrak{h}}=0$ on $\mathfrak{h} \times \mathfrak{h}$ for all strongly symmetric algebras.

The next proposition supplements Theorem 4.22. It shows that $j=J^{1 / 2}$ is essentially the only choice allowing some symmetric spaces to be special.

Proposition 4.23 Let $e_{j}$ denote the $(j, \Phi)$ - function, where $\Phi$ is constructed from universal Lie series $A$ and $C$ (see 4.6.4). Assume $e_{j}$ is identically 1. Then $\operatorname{tr}_{\mathfrak{h}}$ ad $\mathfrak{h}_{*}=0$ and there exists a linear form $\xi \in \mathfrak{s}^{*}$ such that $j(X)=$ $J(X)^{1 / 2} e^{<\xi, X>}$ for all $X$. Thus $e_{j}=e_{J^{1 / 2}}=1$, and $j=J^{1 / 2}$ if $j$ is even.

Proof. By Proposition 4.36 below the $\left(J^{1 / 2}, \Phi\right)$-function satisfies

$$
\log e_{J^{1 / 2}}(X, Y)=\frac{1}{2} \operatorname{tr}_{\mathfrak{h}} \text { ad }\left(\left(\operatorname{th} \frac{x}{2}\right) Y\right)+O\left(Y^{2}\right) .
$$

Let $f$ be defined by $j(X)=J^{1 / 2}(X) e^{f(X)}$, an analytic function on a neighborhood of 0 in $\mathfrak{s}$ with $f(0)=0$. If

$$
\log e_{j}(X, Y)=\log e_{J^{1 / 2}}(X, Y)+f(X)+f(Y)-f(X+Y)
$$

vanishes identically near the origin, the first order terms in $Y$ give

$$
\frac{1}{2} \operatorname{tr}_{\mathfrak{h}} \text { ad }\left(\left(\operatorname{th} \frac{x}{2}\right) Y\right)=(D f(X)-D f(0)) Y .
$$

At first order in $X$ this implies $\frac{1}{4} \operatorname{tr}_{\mathfrak{h}} \operatorname{ad}[X, Y]=D^{2} f(0)(X, Y)$ and both sides must vanish identically, the right being symmetric and the left skewsymmetric with respect to $(X, Y)$. Thus $\operatorname{tr}_{\mathfrak{h}} \operatorname{ad}[\mathfrak{s}, \mathfrak{s}]=0$. Besides

$$
\left(\operatorname{th} \frac{x}{2}\right) Y=\left[X, \frac{\operatorname{th} x / 2}{x} Y\right]
$$


belongs to $[\mathfrak{s}, \mathfrak{s}]$ and the left-hand side of $(4.42)$ vanishes, hence $D f(X)=$ $D f(0)$. Since $f(0)=0, f$ is a linear form on $\mathfrak{s}$ and $e_{j}=e_{J^{1 / 2}}$. If $f$ is even, it must vanish identically and $j=J^{1 / 2}$.

Theorem 4.24 (symmetry of $e$-functions) Let e be the $(j, F, G)-$ function of a symmetric space, constructed from functions $F, G$ satisfying the Kashiwara-Vergne equation (KV1) and $G(X, Y)=F(-Y,-X)$. Then

(i) The function $e_{\omega}(X, Y):=e(X, Y)\left(\operatorname{det}_{\mathfrak{h}} \operatorname{Ad}(h \circ \Phi(X, Y))^{-1 / 2}\right.$ is symmetric on $\Omega$, that is $e_{\omega}(Y, X)=e_{\omega}(X, Y)$.

(ii) $e(X, Y)=e(Y, X)$ for all $(X, Y) \in \Omega$ if and only if $\operatorname{tr}_{\mathfrak{h}} \operatorname{ad}_{\mathfrak{h}}=0$.

As shown in the next section $e_{\omega}$ is the natural $e$-function for the line bundle of half-densities.

By Lemma 4.11 the symmetry of $e$ in (ii) holds true if (and only if for simply connected $G$ ) the space $S=G / H$ has a $G$-semi-invariant measure. By Theorem 3.9 this symmetry implies the commutativity of the algebra $\mathbb{D}(S)$.

Proof. The skew-symmetry of $C(X, Y)$ (Proposition 4.8) is the key to the proof. In view of the definition (4.23) of $e$ the factor $j$ plays no role here and we may as well assume $j=J^{1 / 2}$ and use Proposition 4.19.

(i) Let $e_{\omega, t}:=e_{t} \cdot\left(\operatorname{det}_{\mathfrak{h}} \operatorname{Ad} \widehat{h}_{t}\right)^{-1 / 2}$ with $\widehat{h}_{t}(X, Y)=h \circ \Phi(t X, t Y)$. Writing as before $\overline{A_{t}}(X, Y)=A_{t}(Y, X)$ etc. we know from the proof of Proposition 4.10 that $\widehat{h}_{t}^{-1} \partial_{t} \widehat{h}_{t}=\left(\bar{A}_{t}-B_{t}\right) \circ \Phi_{t}$, therefore

$$
\partial_{t} \log \left(\operatorname{det}_{\mathfrak{h}} \operatorname{Ad} \widehat{h}_{t}\right)^{-1 / 2}=-\frac{1}{2} \operatorname{tr}_{\mathfrak{h}} \operatorname{ad}\left(\bar{A}_{t}-B_{t}\right) \circ \Phi_{t} .
$$

Besides we have by (4.32), (4.35) and (4.36)

$$
\partial_{t} \log e_{t}=\mathcal{J}-\frac{1}{2} \operatorname{tr}_{\mathfrak{h}}\left(-\partial_{X} C_{t} \circ x+\partial_{Y} C_{t} \circ y+\operatorname{ad}\left(A_{t}+B_{t}\right)\right) \circ \Phi_{t}
$$

with $\mathcal{J}(X, Y)=\mathcal{J}(Y, X)$. But $C_{t}=B_{t}-A_{t}=-\overline{C_{t}}$ by Proposition 4.8 (ii), therefore $\partial_{Y} C_{t}=-\partial_{Y} \overline{C_{t}}=-\overline{\partial_{X} C_{t}}$ and $-\partial_{X} C_{t} \circ x+\partial_{Y} C_{t} \circ y$ is invariant under permutation of $X$ and $Y$. From this we infer an equality of the form

$$
\partial_{t} \log e_{\omega, t}(X, Y)=\operatorname{tr}_{\mathfrak{h}}\left(u_{t}(X, Y)+v_{t}\left(\Phi_{t}(X, Y)\right)\right)
$$

where $u_{t}, v_{t}$ are non-commutative series in ad $X, \operatorname{ad} Y$ such that $u_{t}(X, Y)=$ $u_{t}(Y, X)$ and $v_{t}(X, Y)=v_{t}(Y, X)$.

The map $\Phi_{t}$ is not symmetric but, in view of Proposition 4.10 (ii) with $\left(X_{t}, Y_{t}\right)=\Phi_{t}(X, Y)=t^{-1} \Phi(t X, t Y)$

$$
\begin{aligned}
v_{t}\left(\Phi_{t}(Y, X)\right) & =v_{t}\left(\widehat{h}_{t} \cdot\left(Y_{t}, X_{t}\right)\right)=\operatorname{Ad} \widehat{h}_{t} \circ v_{t}\left(Y_{t}, X_{t}\right) \circ \operatorname{Ad} \widehat{h}_{t}^{-1} \\
& =\operatorname{Ad} \widehat{h}_{t} \circ v_{t}\left(\Phi_{t}(X, Y)\right) \circ \operatorname{Ad} \widehat{h}_{t}^{-1}
\end{aligned}
$$

Thus $\operatorname{tr}_{\mathfrak{h}} v_{t}\left(\Phi_{t}(X, Y)\right)$ is symmetric and (i) is proved. 
(ii) Since $\bar{A}_{t}-B_{t}$ belongs to $\mathfrak{h}_{*}$ we have $\operatorname{det}_{\mathfrak{h}} \operatorname{Ad} \widehat{h}_{t}=1$ if $\operatorname{tr}_{\mathfrak{h}}$ ad $\mathfrak{h}_{*}=0$ and (i) implies the symmetry of $e$.

Conversely the Taylor expansion $\log e(X, Y)=\frac{1}{4} \operatorname{tr}_{\mathfrak{h}} \operatorname{ad}[X, Y]+O_{4}$ (Proposition 4.31 below) shows that the symmetry of $e$ on $\Omega$ implies $\operatorname{tr}_{\mathfrak{h}}$ ad $\mathfrak{h}_{*}=0$.

\subsection{Extension to Line Bundles}

In this section we extend the construction of $e$ and the above results to line bundles over symmetric spaces, using the notation $L_{\chi},(x, z)$ etc. of Sections 2.2 and 3.8. The previous study coresponds to the trivial character $\chi=1$. The symbol $\sim$, resp. $*$, now denotes the transfer map, resp. the convolution, for the line bundle. Our first theorem generalizes Theorem 4.12.

Theorem 4.25 Let $S=G / H$ be a symmetric space and $\chi$ a character of $H$. Let $u, v$ be $H$-invariant distributions on open subsets of $\mathfrak{s}$ and let $f \in C^{\infty}(\mathfrak{s})$, with suitable supports. Then

$$
\langle\widetilde{u} * \widetilde{v}, \widetilde{f}\rangle=\left\langle u(X) \otimes v(Y), e_{\chi}(X, Y) f(X+Y)\right\rangle
$$

with

$$
e_{\chi}(X, Y):=e(X, Y) \chi(h \circ \Phi(X, Y))^{-1}
$$

where $e$ is the $(j, \Phi)$ - function (4.23) of $S$.

The convolution equality holds in particular if $u, v$ are $H$-invariant distributions on open subsets of $\mathfrak{s}$, with $\operatorname{supp} v=\{0\}, f \in C^{\infty}(\mathfrak{s})$, and $\operatorname{supp} u \cap$ supp $f \cap \Omega_{1}$ is compact.

Here again $\Omega_{1}=\{X \in \mathfrak{s} \mid(X, 0) \in \Omega\}$. More generally, the result holds under the assumption on supports specified at the end of the proof of Theorem 4.12, but the special case mentioned here suffices for the application to invariant differential operators. We shall call $(4.43)$ the $(j, \Phi, \chi)-$ function of the line bundle $L_{\chi}$.

Proof. From the definitions of $\sim$ and $*$ we have

$$
\langle\widetilde{u} * \widetilde{v}, \tilde{f}\rangle=\left\langle u(X), j(X)\left\langle\widetilde{v}, \tilde{f}_{\exp X}\right\rangle\right\rangle,
$$

where the section $\widetilde{f}_{\exp X}$ of the line bundle is given by $\widetilde{f}_{\exp X}(\operatorname{Exp} Y)=$ $e^{-X} \cdot \tilde{f}\left(e^{X} e^{Y} H\right)$. Using the decomposition $e^{X} e^{Y}=e^{Z} h$ with $Z=Z(X, Y)$ and $h=h(X, Y)$ we obtain

$$
\begin{aligned}
\tilde{f}_{\exp X}(\operatorname{Exp} Y) & =e^{-X} \cdot \underline{\left(e^{Z}, j(Z)^{-1} f(Z)\right)}=\underline{\left(e^{Y} h^{-1}, j(Z)^{-1} f(Z)\right)} \\
& =\underline{\left(e^{Y}, \chi(h)^{-1} j(Z)^{-1} f(Z)\right)}
\end{aligned}
$$


and it follows that

$$
\langle\widetilde{u} * \widetilde{v}, \widetilde{f}\rangle=\left\langle u(X) \otimes v(Y), \frac{j(X) j(Y)}{j(Z(X, Y))} \chi(h(X, Y))^{-1} f(Z(X, Y))\right\rangle .
$$

We can then use (4.25) with $g(X, Y):=e_{\chi}(X, Y) f(X+Y)$ and $e_{\chi}$ defined by (4.43). The resulting equality is $\left\langle u \otimes v, g_{1}\right\rangle=\left\langle u \otimes v, g_{0}\right\rangle$, with

$$
\begin{aligned}
g_{1}(X, Y) & =\frac{j(X) j(Y)}{j(Z(X, Y))} \chi(h(X, Y))^{-1} f(Z(X, Y)) \\
g_{0}(X, Y) & =e_{\chi}(X, Y) f(X+Y),
\end{aligned}
$$

which implies our claim.

Given a character $\chi$ of $H$ we denote by $\chi^{\prime}$ its differential at the origin, which is a character of the Lie algebra $\mathfrak{h}$ (that is $\chi^{\prime}([\mathfrak{h}, \mathfrak{h}])=0$ ); thus $\chi\left(e^{V}\right)=$ $e^{\chi^{\prime}(V)}$ for $V \in \mathfrak{h}$. A character of $\mathfrak{h}$ extends to a character of $\mathfrak{g}=\mathfrak{h} \oplus \mathfrak{s}$ if and only if it vanishes on the ideal $\mathfrak{h}_{*}=[\mathfrak{s}, \mathfrak{s}]$ of $\mathfrak{h}$ : indeed extending it by 0 on $\mathfrak{s}$ proves the «if»part, and the converse is obvious.

Proposition 4.26 The function $e_{\chi}$ defined by (4.43) has the following properties.

(i) It is analytic on $\Omega$, strictly positive, and $e_{\chi}(X, Y)=e_{\chi}(h \cdot X, h \cdot Y)$ for all $h \in H$. Besides $e_{\chi}(X, Y)=e_{\chi}(-X,-Y)$ if $j$ is even.

(ii) Given two characters $\chi_{1}, \chi_{2}$ of $H$ we have $e_{\chi_{1}}=e_{\chi_{2}}$ if and only if the character $\chi_{1}^{\prime}-\chi_{2}^{\prime}$ of $\mathfrak{h}$ extends to a character of $\mathfrak{g}$.

Under the assumption of (ii) the line bundles $L_{\chi_{1}}$ and $L_{\chi_{2}}$ are isomorphic. Proof. (i) is clear by Proposition $4.2(i)$, Theorem $4.6(i i)$ and Remark $(d)$ after Theorem 4.12.

(ii) Assume $\chi_{1}^{\prime}-\chi_{2}^{\prime}$ extends to $\mathfrak{g}$. Then, $G$ being simply connected, $\chi_{1} \chi_{2}^{-1}$ extends to a character of $G$. By Proposition $4.2(i v)$ it follows that $\chi_{1}(h \circ \Phi)=$ $\chi_{2}(h \circ \Phi)$ hence $e_{\chi_{1}}=e_{\chi_{2}}$.

Conversely $e_{\chi_{1}}=e_{\chi_{2}}$ implies $\chi_{1} \chi_{2}^{-1}(h \circ \Phi(X, Y))=1$ for all $(X, Y) \in \Omega$. But $h \circ \Phi(X, Y)=\exp \left(\frac{1}{2}[X, Y]+O_{4}\right)$ by Lemma 4.30 , therefore $\left(\chi_{1}^{\prime}-\chi_{2}^{\prime}\right)[X, Y]=$ 0 for all $X, Y \in \mathfrak{s}$. The character $\chi_{1}^{\prime}-\chi_{2}^{\prime}$ of $\mathfrak{h}$, which vanishes on $[\mathfrak{s}, \mathfrak{s}]$, extends to a character of $\mathfrak{g}$.

Let us consider the following characters of $H$, resp. $\mathfrak{h}$,

$$
\omega(h):=\left(\operatorname{det}_{\mathfrak{h}} \operatorname{Ad} h\right)^{1 / 2}, \text { resp. } \omega^{\prime}:=\frac{1}{2} \operatorname{tr}_{\mathfrak{h}} \text { ad } .
$$

Since $\operatorname{det}_{\mathfrak{g}} \operatorname{Ad} h=\left(\operatorname{det}_{\mathfrak{h}} \operatorname{Ad} h\right)\left(\operatorname{det}_{\mathfrak{g} / \mathfrak{h}} \operatorname{Ad} h\right)$ extends to a character of $G$ we see that, up to isomorphism, $\omega$ defines the same bundle as $\left(\operatorname{det}_{\mathfrak{g} / \mathfrak{h}} \operatorname{Ad} h\right)^{-1 / 2}$, that is the bundle of half-densities on $G / H$ (Section 2.2.1). 
The line bundle $L_{\chi}$ is called special if the convolution equality of Theorem 4.25 holds with $e_{\chi}=1$. In the next theorem we specialize to $j=J^{1 / 2}$, an assumption motivated by an easy extension of Proposition 4.23: if $j$ is even, $A, C$ are universal and the $(j, \Phi, \chi)$-function of a line bundle is identically 1 , then $j=J^{1 / 2}$.

Theorem 4.27 (special line bundles) Let $e_{\chi}$ be the $\left(J^{1 / 2}, \Phi, \chi\right)-$ function of a line bundle $L_{\chi}$, where $\Phi$ is constructed from Lie series $F, G$ satisfying the Kashiwara-Vergne equations (KV1) and (KV2). Then

(i) $e_{\chi}$ is identically 1 on $\Omega$ if and only if $e_{\omega}$ is identically 1 on $\Omega$ and $\chi^{\prime}-\omega^{\prime}$ extends to a character of $\mathfrak{g}$.

(ii) $e_{\chi}(X, Y)=1$ if $(X, Y) \in \Omega$, if $X, Y$ belong to a solvable Lie subalgebra of $\mathfrak{g}$ and $\chi^{\prime}-\omega^{\prime}$ extends to a character of $\mathfrak{g}$.

(iii) $e_{\chi}$ is identically 1 on $\Omega$ if the space $G / H$ is strongly symmetric and $\chi^{\prime}-\omega^{\prime}$ extends to a character of $\mathfrak{g}$.

Thus, among bundles equipped with $e_{\chi}$ defined by (4.43), only the bundle $L_{\omega}$ of half-densities can be special (up to isomorphism). It actually is if $G$ is a solvable Lie group or if $G / H$ is strongly symmetric. See Proposition 3.28 for some properties of special bundles.

Proof. (i) The "if" part follows from Proposition 4.26 (ii). Conversely the expansion $\log e_{\chi}(X, Y)=\frac{1}{2}\left(\omega^{\prime}-\chi^{\prime}\right)[X, Y]+O_{4}$ (Proposition 4.31) shows that $e_{\chi}=1$ implies the vanishing of $\chi^{\prime}-\omega^{\prime}$ on $[\mathfrak{s}, \mathfrak{s}]$. Thus it extends to a character of $\mathfrak{g}$ and $e_{\omega}=e_{\chi}=1$ by Proposition 4.26 (ii) again.

(ii) By Theorem $4.22(i)$ we have $e(X, Y)=1$ and it remains to show that

$$
\chi(h \circ \Phi(X, Y))=1 .
$$

We know from the proof of Proposition 4.10 that $\widehat{h}_{t}(X, Y):=h \circ \Phi(t X, t Y)$ solves the differential equation

$$
\widehat{h}_{t}^{-1} \partial_{t} \widehat{h}_{t}=H_{t} \circ \Phi_{t}
$$

and the right-hand side belongs to $[\mathfrak{s}, \mathfrak{s}]$ (note that the assumption $G(X, Y)=$ $F(-Y,-X)$ of Proposition 4.10 was not used for that). Therefore $h \circ \Phi(X, Y)$ belongs to $H_{*}$, the connected Lie subgroup of $H$ generated by $\exp [\mathfrak{s}, \mathfrak{s}]$. But for $V \in[\mathfrak{s}, \mathfrak{s}]$ we have $\chi\left(e^{V}\right)=e^{\chi^{\prime}(V)}=e^{\omega^{\prime}(V)}$ since $\chi^{\prime}-\omega^{\prime}$ vanishes on $[\mathfrak{s}, \mathfrak{s}]$, and $\omega^{\prime}(V)=\frac{1}{2} \operatorname{tr}_{\mathfrak{h}}$ ad $V=0$ since ad $V$ is nilpotent as in the proof of Theorem 4.22 .

(iii) The proof is similar to (ii): here $e=1$ and $\operatorname{tr}_{\mathfrak{h}} \operatorname{ad}[\mathfrak{s}, \mathfrak{s}]=0$ by Theorem 4.22 , hence $\chi^{\prime}(V)=\omega^{\prime}(V)=0$ and $\chi$ is trivial on $H_{*}$.

Theorem 4.28 (symmetry of $\left.e_{\chi}\right)$ Let $e_{\chi}$ be the $(j, \Phi, \chi)-$ function of a line bundle $L_{\chi}$, where $\Phi$ is constructed from functions $F, G$ satisfying the Kashiwara-Vergne equation (KV1) and $G(X, Y)=F(-Y,-X)$. Then

(i) $e_{\chi}(Y, X)=e_{\omega^{2} \chi^{-1}}(X, Y)$ for $(X, Y) \in \Omega$

(ii) $e_{\chi}$ is symmetric on $\Omega$, that is $e_{\chi}(X, Y)=e_{\chi}(Y, X)$, if and only if $e_{\chi}=e_{\omega}$, that is if the character $\chi^{\prime}-\omega^{\prime}$ of $\mathfrak{h}$ extends to a character of $\mathfrak{g}$. 
Remarks. (a) The bundle of half-densities is thus (up to isomorphism) the only line bundle over $S$ with a symmetric function $e_{\chi}$. By $(i i)$ the convolution of $H$-invariant distributions and the algebra $\mathbb{D}\left(L_{\chi}\right)$ (see Theorem 3.27) are commutative whenever $\chi^{\prime}-\omega^{\prime}$ extends to a character of $\mathfrak{g}$; the latter result was originally proved by Duflo [19].

(b) Combining Theorem 3.27 (iii) with Proposition 4.31 the following necessary condition is easily obtained (cf. [45] p. 125): if $\mathbb{D}\left(L_{\chi}\right)$ is commutative, then $\left(\chi^{\prime}-\omega^{\prime}\right)[X, Y]=0$ whenever $X=D P(\xi)$ and $Y=D Q(\xi)$ with $P, Q \in S(\mathfrak{s})^{H}$ and $\xi \in \mathfrak{s}^{*}$. The differentials $D P(\xi), D Q(\xi)$ are here identified with elements of $\mathfrak{s}$.

(c) For the trivial bundle $(\chi=1)$, (ii) shows that $e_{\chi}=e$ is symmetric if and only if $\omega^{\prime}=\frac{1}{2} \operatorname{tr}_{\mathfrak{h}}$ ad vanishes on $[\mathfrak{s}, \mathfrak{s}]$. Thus Theorem 4.28 generalizes Theorem 4.24.

Proof. (i) By (4.43) we have $e_{\chi}(X, Y)=e_{\omega}(X, Y)\left(\omega \chi^{-1}\right)(h \circ \Phi(X, Y))$. Under permutation of $X$ and $Y$ the factor $e_{\omega}$ is invariant (Theorem 4.24 (i)) whereas the second factor changes into its inverse (Proposition $4.10(i)$ ), hence

$$
e_{\chi}(Y, X)=e_{\omega}(X, Y)\left(\omega^{-1} \chi\right)(h \circ \Phi(X, Y))=e_{\omega^{2} \chi^{-1}}(X, Y) .
$$

(ii) By (i) $e_{\chi}$ is symmetric if and only if $e_{\chi}=e_{\omega^{2} \chi^{-1}}$, in other words if $\chi^{\prime}-\left(2 \omega^{\prime}-\chi^{\prime}\right)=2\left(\chi^{\prime}-\omega^{\prime}\right)$ extends to a character of $\mathfrak{g}$ (Proposition 4.26 (ii)). This is equivalent to the vanishing of $\chi^{\prime}-\omega^{\prime}$ on $[\mathfrak{s}, \mathfrak{s}]$, or else to $e_{\chi}=e_{\omega}$.

\subsection{Taylor Expansions}

In this section we expand all important expressions considered in the chapter, first up to order 4 or 5 with respect to $(X, Y)$, then up to an arbitrary order with respect to $X$ but up to order 1 with respect to $Y$. These expansions aim at giving a better understanding of the functions we have introduced, and possibly testing conjectures about their properties. They are also used in the course of several proofs in 4.4 and 4.5 .

In the following $O_{n}$ means terms of order $\geq n$ with respect to $(X, Y)$ - not to be confused with the degree with respect to $t$ in expressions like $A_{t}(X, Y)=t^{-1} A(t X, t Y)$.

As usual we write $x=\operatorname{ad} X, y=\operatorname{ad} Y$. All terms in our expansions will be written as $x^{\alpha_{1}} y^{\beta_{1}} \ldots x^{\alpha_{n}} Y$, which is short and convenient but not unique: for instance $[[X, Y],[X, Y]]=0$ gives $x y x Y=y x^{2} Y$ because ad $[X, Y]=x y-y x$ and $[X, Y]=x Y$. Such relations will be taken into account.

\subsubsection{Expansions of $Z, h, A, B, C$}

Recall the definitions $Z(X, Y)=\frac{1}{2} e^{-x} \log \left(e^{2 X} e^{2 Y}\right), h(X, Y)=e^{-Z(X, Y)} e^{X} e^{Y}$ (Proposition 4.2). Moreover $A, B, C$ are even Lie series such that $B=A+C$ 
and

$$
Z(Y, X)=(\operatorname{ch} y)(X+Y)-(\operatorname{ch} y \operatorname{sh} x+\operatorname{sh} y \operatorname{ch} x) A(X, Y)-(\operatorname{sh} y) C(X, Y)
$$

for $(X, Y) \in \mathcal{U}$ (Proposition $4.5(i))$.

Lemma 4.29 For $X, Y$ near the origin of $\mathfrak{g} \times \mathfrak{g}$ we have

$$
\begin{gathered}
Z(X, Y)=X+Y-\frac{1}{6}\left(x^{2}+2 y x\right) Y+ \\
+\frac{1}{360}\left(7 x^{4}+16 y x^{3}+12 x y x^{2}+48 y^{2} x^{2}-16 x y^{2} x+8 y^{3} x\right) Y+O_{7} \\
h(X, Y)=\exp \left(\frac{1}{2} x Y-\frac{1}{24}\left(x^{2}+3 x y+y^{2}\right) x Y+O_{6}\right) \\
A(X, Y)=-\frac{1}{3} x Y+\frac{1}{90}\left(7 x^{2}+12 y x+4 y^{2}\right) x Y+O_{6} \\
C(X, Y)=-\frac{1}{3} x Y+\frac{1}{45}\left(2 x^{2}+3 y x+2 y^{2}\right) x Y+O_{6}
\end{gathered}
$$

Proof. From the classical Campbell-Hausdorff expansion up to order 5:

$$
\begin{aligned}
\log \left(e^{X} e^{Y}\right) & =X+Y+\frac{1}{2} x Y+\frac{1}{12} x^{2} Y-\frac{1}{12} y x Y-\frac{1}{24} y x^{2} Y+ \\
& +\frac{1}{720}\left(-x^{4}+2 y x^{3}-6 x y x^{2}+6 y^{2} x^{2}-2 x y^{2} x+y^{3} x\right) Y+O_{6}
\end{aligned}
$$

the expansion of $Z(X, Y)$ is easily obtained. Since $Z(-X,-Y)=-Z(X, Y)$ all even terms may be neglected during the calculations.

The expansion of $h=e^{-Z}\left(e^{X} e^{Y}\right)$ follows, applying twice the CampbellHausdorff formula up to order 4 .

It turns out that the expansion of $Z$ together with (4.44) uniquely determine $A$ and $C$ up to order 4. Indeed the expansions (4.45) and (4.46) are easily obtained by identification: we may look for

$$
A=a x Y+b x^{3} Y+c y x^{2} Y+d y^{2} x Y+O_{6}
$$

(where $a, b, c, d$ are unknown coefficients) and a similar expression for $C$; all terms have different degrees with respect to $X$ and to $Y$, and $x y x Y=$ $y x^{2} Y$. The result drops down from (4.44) and the expansion of $Z$ after some computations. Note that (4.46) implies $C(Y, X)=-C(X, Y)$ necessarily, up to order 4 at least.

Then $A_{t}=t^{-1} A(t X, t Y)$ and $B_{t}=A_{t}+C_{t}$ are given by

$$
\begin{aligned}
& A_{t}(X, Y)=-\frac{t}{3} x Y+\frac{t^{3}}{90}\left(7 x^{3}+12 y x^{2}+4 y^{2} x\right) Y+O_{6} \\
& B_{t}(X, Y)=-\frac{2 t}{3} x Y+\frac{t^{3}}{90}\left(11 x^{3}+18 y x^{2}+8 y^{2} x\right) Y+O_{6} .
\end{aligned}
$$




\subsubsection{Expansion of $\Phi$}

Let $\Phi$ and $\Phi_{t}$ be given by Theorem 4.6, from $A, C$ satisfying (4.44) and $B=A+C$, and let $c_{t}=a_{t}^{-1} b_{t}$.

Lemma 4.30 In a neighborhood of the origin of $\mathfrak{s} \times \mathfrak{s}, \Phi_{t}(X, Y)=\left(X_{t}, Y_{t}\right)$ and $U_{t}=c_{t}(X, Y) \cdot Y$ expand as

$$
\begin{aligned}
X_{t} & =X+\frac{t^{2}}{6} x^{2} Y+\frac{t^{4}}{360}\left(-7 x^{4}-12 x y x^{2}+5 y x^{3}-4 x y^{2} x\right) Y+O_{7} \\
Y_{t} & =Y+\frac{t^{2}}{3} y x Y+\frac{t^{4}}{360}\left(-11 y x^{3}+12 y^{2} x^{2}-20 x y^{2} x-8 y^{3} x\right) Y+O_{7} \\
U_{t} & =Y+\frac{t^{2}}{6} y x Y+\frac{t^{4}}{360}\left(-4 y x^{3}+4 y^{2} x^{2}-5 x y^{2} x-4 y^{3} x\right) Y+O_{7} .
\end{aligned}
$$

Also

$$
\begin{aligned}
& c_{t}(X, Y)=\exp \left(-\frac{t^{2}}{6} x Y+\frac{t^{4}}{360}\left(4 x^{2}+x y+4 y^{2}\right) x Y+O_{6}\right) \\
& h \circ \Phi(X, Y)=\exp \left(\frac{1}{2} x Y-\frac{1}{24}\left(x^{2}+x y+y^{2}\right) x Y+O_{6}\right) .
\end{aligned}
$$

Proof. In view of Theorem 4.6 we must solve

$$
\begin{aligned}
\partial_{t} X_{t} & =\left[A_{t}\left(X_{t}, Y_{t}\right), X_{t}\right], X_{0}=X \\
\partial_{t} Y_{t} & =\left[B_{t}\left(X_{t}, Y_{t}\right), Y_{t}\right], Y_{0}=Y
\end{aligned}
$$

in the form of $X_{t}=X+t^{2} X_{3}+t^{4} X_{5}+O_{7}, Y_{t}=Y+t^{2} Y_{3}+t^{4} Y_{5}+O_{7}$. Taking into account the identities

$$
\begin{aligned}
& x^{2} y x Y=x y x^{2} Y, \operatorname{ad}\left(x^{2} Y\right)=x^{2} y-2 x y x+y x^{2} \\
& y x y x Y=y^{2} x^{2} Y, \operatorname{ad}\left(y^{2} X\right)=y^{2} x-2 y x y+x y^{2}
\end{aligned}
$$

and the above expansions of $A_{t}, B_{t}$, the result is easily obtained by identification.

The expansion of $U_{t}$ follows similarly from $\partial_{t} U_{t}=\left[C_{t}\left(X, U_{t}\right), U_{t}\right], U_{0}=Y$ (Lemma 4.7).

According to Proposition 4.8, $c_{t}=\exp \gamma_{t}$ with $\gamma_{t} \in \mathfrak{h}_{*}=[\mathfrak{s}, \mathfrak{s}]$ and $\gamma_{t}(-X,-Y)=$ $\gamma_{t}(X, Y)$, thus

$$
\gamma_{t}=t^{2} a x Y+t^{4}\left(b x^{2}+c x y+d y^{2}\right) x Y+O_{6}
$$

where $a, b, c, d$ are scalars. These coefficients may be obtained by identification of $c_{t} \cdot Y=\exp \left(\operatorname{ad} \gamma_{t}\right) Y$ with the above expansion of $U_{t}$, or else from the differential equation $\left(D_{e} R_{c_{t}}\right)^{-1} \partial_{t} c_{t}=C_{t}\left(X, U_{t}\right)$ (Lemma 4.7) which is equivalent to

$$
\frac{e^{\operatorname{ad} \gamma_{t}}-1}{\operatorname{ad} \gamma_{t}} \gamma_{t}^{\prime}=\left(1+\frac{1}{2} \operatorname{ad} \gamma_{t}+\frac{1}{6}\left(\operatorname{ad} \gamma_{t}\right)^{2}+\cdots\right) \gamma_{t}^{\prime}=C_{t}\left(X, U_{t}\right) .
$$


The definition of $h$ and the properties of $\Phi$ imply

$$
h \circ \Phi(X, Y)=\left(e^{-X-Y} e^{X_{1}}\right) e^{Y_{1}} .
$$

Knowing that

$$
X_{1}=X+\frac{1}{6} x^{2} Y+O_{5}, Y_{1}=Y+\frac{1}{3} y x Y+O_{5} .
$$

the result for $h \circ \Phi$ follows from the classical Campbell-Hausdorff formula, applied twice up to order 4 . Note that the remainder is actually $\mathrm{O}_{6}$ since $h \circ \Phi$ is an even function.

\subsubsection{Expansions of $e$ and $e_{\chi}$}

We can now expand the $\left(J^{1 / 2}, \Phi\right)$ - function defined by $(4.23)$ with $j=$ $J^{1 / 2}$. Let us introduce $T:=[X, Y]=x Y$ and the Killing forms $B_{\mathfrak{g}}(U, V):=$ $\operatorname{tr}_{\mathfrak{g}}(\operatorname{ad} U \operatorname{ad} V)$, resp. $B_{\mathfrak{h}}(U, V):=\operatorname{tr}_{\mathfrak{h}}(\operatorname{ad} U \operatorname{ad} V)$, of the Lie algebras $\mathfrak{g}$, resp. $\mathfrak{h}$. For line bundles we recall the notation $\chi^{\prime}$ (the differential at the origin of the character $\chi$ of $H), \omega^{\prime}=\frac{1}{2} \operatorname{tr}_{\mathfrak{h}}$ ad (that is $\chi^{\prime}$ for the bundle of half-densities) and the definition $(4.43) e_{\chi}=e \cdot \chi(h \circ \Phi)^{-1}$.

Proposition 4.31 In a neighborhood of the origin of $\mathfrak{s} \times \mathfrak{s}$ the logarithm of the $\left(J^{1 / 2}, \Phi\right)$ - function e expands as

$\log e(X, Y)=\frac{1}{4} \operatorname{tr}_{\mathfrak{h}}$ ad $\left(T-\frac{1}{12}\left(x^{2}+x y+y^{2}\right) T\right)-\frac{1}{240}\left(B_{\mathfrak{g}}-2 B_{\mathfrak{h}}\right)(T, T)+O_{6}$.

More generally, for any character $\chi$ of $H$,

$\log e_{\chi}(X, Y)=\frac{1}{2}\left(\omega^{\prime}-\chi^{\prime}\right)\left(T-\frac{1}{12}\left(x^{2}+x y+y^{2}\right) T\right)-\frac{1}{240}\left(B_{\mathfrak{g}}-2 B_{\mathfrak{h}}\right)(T, T)+O_{6}$.

Note that the first term of $\log e$ vanishes if $\operatorname{tr}_{\mathfrak{h}}$ ad $\mathfrak{h}_{*}=0$. More generally, the first term of $\log e_{\chi}$ vanishes if $\chi^{\prime}-\omega^{\prime}$ extends to a character of $\mathfrak{g}$.

Proof. By Proposition $4.19(i)$ :

$$
\begin{aligned}
\left.\partial_{t} \log e_{t}(X, Y)\right|_{t=1}=\frac{1}{2} \operatorname{tr}_{\mathfrak{h}}(x \operatorname{coth} x+ & y \operatorname{coth} y-(x+y) \operatorname{coth}(x+y)-1)- \\
& -\operatorname{tr}_{\mathfrak{h}}\left(\left(\partial_{Y} C\right) \circ y+\operatorname{ad} A\right)\left(X_{1}, Y_{1}\right) .
\end{aligned}
$$

Having computed this for $t=1$ the general case will follow, observing that $\log e_{s t}(X, Y)=\log e_{s}(t X, t Y)$ hence

$$
\partial_{t} \log e_{t}(X, Y)=t^{-1}\left(\left.\partial_{s} \log e_{s}\right|_{s=1}\right)(t X, t Y)
$$

by taking the derivative with respect to $s$ at $s=1$; then $\log e_{t}(X, Y)=$ $\log e(t X, t Y)$ is obtained by integration. To perform the calculations one uses the following: 
- $x \operatorname{coth} x=1+\frac{x^{2}}{3}-\frac{x^{4}}{45}+O_{6}$

- $x^{2} y x \sim y x^{3}$ (and similar relations), where $a \sim b$ means $\operatorname{tr}_{\mathfrak{h}} a=\operatorname{tr}_{\mathfrak{h}} b$

- $y^{2} x Y=-\operatorname{ad}(y x Y) Y=-y \operatorname{ad}(x Y) Y$, implying

$$
\partial_{Y}\left(y^{2} x Y\right)=y^{2} x-\operatorname{ad}(y x Y)-y \operatorname{ad}(x Y)
$$

(and similar relations), to compute $\partial_{Y} C$

- $\operatorname{ad}\left(x^{3} Y\right)=x^{3} y-3 x^{2} y x+3 x y x^{2}-y x^{3} \sim 4\left(x^{3} y-y x^{3}\right)$ (and similar relations) to compute ad $A$

- replace $x$ by ad $X_{1}=x+\frac{1}{6} \operatorname{ad}\left(x^{2} Y\right)+O_{5}$ and $y$ by ad $Y_{1}=y+$ $\frac{1}{3} \operatorname{ad}(y x Y)+O_{5}$ in view of Lemma 4.30.

We skip the details, lengthy but easy. One checks that

$$
\begin{aligned}
\left(B_{\mathfrak{g}}-2 B_{\mathfrak{h}}\right)(T, T) & =2 \operatorname{tr}_{\mathfrak{h}}\left(x y^{2} x-x^{2} y^{2}\right)=\operatorname{tr}_{\mathfrak{h}}\left(\left[x y^{2}, x\right]+\left[y x^{2}, y\right]\right) \\
\operatorname{ad}\left(\left(x^{2}+x y+y^{2}\right) T\right) & \sim\left[x, 4 y^{3}+y x y\right]-\left[y, 4 x^{3}+x y x\right],
\end{aligned}
$$

and the expansion of $\log e$ can finally be written as claimed.

Moreover $\log \chi(h \circ \Phi)^{-1}=-\chi^{\prime}(\log (h \circ \Phi))$ and the result for $\log e_{\chi}$ follows from the expansion of $h \circ \Phi$ in Lemma 4.30.

In the following examples $|T|^{2}=\operatorname{tr}\left({ }^{t} T T\right)$ denotes the Hilbert-Schmidt norm of a matrix $T$.

Example 1. For the real hyperbolic space $H^{n}(\mathbb{R})=S O_{o}(n, 1) / S O(n)$ Proposition 4.31 gives

$$
\log e(X, Y)=\frac{3-n}{240}|T|^{2}+O_{6}
$$

with $X, Y \in \mathfrak{s}, T=[X, Y] \in \mathfrak{g}=\operatorname{so}(n, 1)$, and this expansion agrees with the example following Theorem 3.23.

Indeed $\operatorname{tr}_{\mathfrak{h}}$ ad $\mathfrak{h}=0$ since $H=S O(n)$ is a compact group. Besides $B_{\mathfrak{h}}(T, T)=$ $(n-2) \operatorname{tr}\left(T^{2}\right)$ by a classical result for the Killing form of $s o(n)$ ([27] p. 189), and we must evaluate $B_{\mathfrak{g}}(T, T)$. Let

$$
X=\left(\begin{array}{cc}
A & V \\
{ }^{t} V & 0
\end{array}\right) \text {, or } X=(A, V) \text { for short },
$$

denote an element of $\mathfrak{g}=s o(n, 1)$, where $A \in s o(n)$ is a skew-symmetric $n \times n$ real matrix and $V \in \mathbb{R}^{n}$ is a column vector. The subalgebra $\mathfrak{h}$ of $\mathfrak{g}$ is defined by $V=0$ and the subspace $\mathfrak{s}$ by $A=0$. Let $H \in \mathfrak{s}$ be defined by $A=0$ and ${ }^{t} V=(1,0, \ldots, 0)$. Computing brackets it is easily checked, with the root space decomposition of $\mathfrak{g}$ defined by the abelian subspace $\mathbb{R} H$, that the eigenvalues of ad $H$ are 1, 0 and -1 , with respective multiplicities $n-1,1$ and $n-1$. Thus $B_{\mathfrak{g}}(H, H)=\operatorname{tr}(\operatorname{ad} H)^{2}=2(n-1)$ and $H$ is a unit vector with respect 
to the norm $\|$.$\| of Section 3.7$ with $p=n-1, q=0$. Extending this remark by $H$-invariance (with $H=S O(n)$ ) to any $X \in \mathfrak{s}$, we see that $\|X\|$ coincides with the canonical norm of $V$ in $\mathbb{R}^{n}$ and $B_{\mathfrak{g}}(X, X)=2(n-1)\|V\|^{2}$.

Now let $X=(0, V), Y=(0, W) \in \mathfrak{s}$; then $T=[X, Y]=(A, 0) \in \mathfrak{h}$ with $A=V^{t} W-W^{t} V$. Observing that $B_{\mathfrak{g}}(T, T)=B_{\mathfrak{g}}(x Y, x Y)=-B_{\mathfrak{g}}\left(Y, x^{2} Y\right)$ and $x^{2} Y=[X, T]=(0,-A V)$, we obtain

$$
\begin{aligned}
B_{\mathfrak{g}}(T, T) & =-2(n-1)(W \cdot(-A V)) \\
& =2(n-1)\left((V \cdot W)^{2}-\|V\|^{2}\|W\|^{2}\right) \\
& =2(n-1)\left((X \cdot Y)^{2}-\|X\|^{2}\|Y\|^{2}\right) .
\end{aligned}
$$

But $2\left((V \cdot W)^{2}-\|V\|^{2}\|W\|^{2}\right)=\operatorname{tr}\left(T^{2}\right)=-|T|^{2}$, therefore

$$
\begin{aligned}
\left(B_{\mathfrak{g}}-2 B_{\mathfrak{h}}\right)(T, T) & =(3-n) \operatorname{tr}\left(T^{2}\right) \\
& =2(n-3)\left(\|X\|^{2}\|Y\|^{2}-(X \cdot Y)^{2}\right) .
\end{aligned}
$$

This implies our claims.

The same expansion of $\log e$ holds for the $n$-sphere $S^{n}=S O(n+1) / S O(n)$ since $B_{\mathfrak{g}}(T, T)=(n-1) \operatorname{tr}\left(T^{2}\right)$ and we still have $B_{\mathfrak{h}}(T, T)=(n-2) \operatorname{tr}\left(T^{2}\right)$.

Example 2. For the symmetric space $S L(n, \mathbb{R}) / S O(n)$ Proposition 4.31 gives

$$
\log e(X, Y)=\frac{1}{60}|T|^{2}+O_{6}
$$

where $|T|^{2}=\operatorname{tr}\left({ }^{t} T T\right)$ is again the Hilbert-Schmidt norm of the matrix $T=$ $[X, Y] \in \mathfrak{g}=\operatorname{sl}(n, \mathbb{R})$.

Indeed, by [27] p. 187 and 189 ,

$$
B_{\mathfrak{g}}(T, T)=2 n \operatorname{tr}\left(T^{2}\right), B_{\mathfrak{h}}(T, T)=(n-2) \operatorname{tr}\left(T^{2}\right),
$$

therefore $\left(B_{\mathfrak{g}}-2 B_{\mathfrak{k}}\right)(T, T)=4 \operatorname{tr}\left(T^{2}\right)=-4|T|^{2}$ for $T \in \operatorname{so}(n)$.

Remark. The expansions in Examples 1 and 2 seem to disagree for the space $H^{2}(\mathbb{R})=S O_{o}(2,1) / S O(2)=S L(2, \mathbb{R}) / S O(2) \ldots$ but it should be noted that the Lie algebra $s o(2,1)$ is isomorphic to $s l(2, \mathbb{R})$ under the map

$$
X=\left(\begin{array}{ccc}
0 & t & u \\
-t & 0 & v \\
u & v & 0
\end{array}\right) \longmapsto X^{\prime}=\frac{1}{2}\left(\begin{array}{cc}
u & v+t \\
v-t & -u
\end{array}\right)
$$

and that $|X|^{2}=4\left|X^{\prime}\right|^{2}$. 


\subsubsection{Expansions of $Z, h, A, C$ Modulo $Y^{2}$}

We now give several expansions up to an arbitrary order with respect to $X$ but up to order 1 only with respect to $Y$. All functions we deal with are analytic in $(X, Y)$ so that $f(X, Y)=O\left(Y^{2}\right)$ will mean: there exist a neighborhood $U$ of the origin in $\mathfrak{g} \times \mathfrak{g}($ or $\mathfrak{s} \times \mathfrak{s})$ and a constant $C>0$ such that $\|f(X, Y)\| \leq C\|Y\|^{2}$ for all $(X, Y) \in U$.

Lie series $A, C$ are called universal solutions of (4.44) if, for any finite dimensional real Lie algebra $\mathfrak{g}$, this equality holds identically in some neighborhood of the origin in $\mathfrak{g}$.

Lemma 4.32 (i) Let $Z(X, Y)$ and $h(X, Y)$ be defined as in Proposition 4.2. Then

$$
\begin{aligned}
& Z(X, Y)=X+\frac{x}{\operatorname{sh} x} Y+O\left(Y^{2}\right) \\
& Z(Y, X)=X+(x \operatorname{coth} x) Y+O\left(Y^{2}\right) \\
& h(X, Y)=\exp \left(\left(\operatorname{th} \frac{x}{2}\right) Y+O\left(Y^{2}\right)\right) .
\end{aligned}
$$

(ii) Let $A$ and $C$ be even Lie series which are universal solutions of (4.44). Then

$$
\begin{aligned}
& A(X, Y)=\frac{1-x \operatorname{coth} x}{\operatorname{sh} x} Y+O\left(Y^{2}\right) \\
& C(X, Y)=\frac{x-\operatorname{sh} x \operatorname{ch} x}{2 \operatorname{sh}^{2} x} Y+O\left(Y^{2}\right)
\end{aligned}
$$

Proof. (i) In view of Proposition $4.2(v)$ we have

$$
\begin{aligned}
& Z(X, Y)=Z(X, 0)+\partial_{Y} Z(X, 0) Y+O\left(Y^{2}\right)=X+\frac{x}{\operatorname{sh} x} Y+O\left(Y^{2}\right) \\
& Z(Y, X)=Z(0, X)+\partial_{X} Z(0, X) Y+O\left(Y^{2}\right)=X+(x \operatorname{coth} x) Y+O\left(Y^{2}\right)
\end{aligned}
$$

for $X, Y$ near the origin of $\mathfrak{g}$. Then $\partial_{Y} h(X, 0)$ is obtained by taking the derivative at $t=0$ of $e^{Z(X, t Y)} h(X, t Y)=e^{X} e^{t Y}$. Since $Z(X, 0)=X$ and $h(X, 0)=e$ it gives

$$
\partial_{Y} h(X, 0) Y=Y-\frac{1-e^{-x}}{x} \partial_{Y} Z(X, 0) Y=\left(1-\frac{1-e^{-x}}{\operatorname{sh} x}\right) Y=\left(\operatorname{th} \frac{x}{2}\right) Y,
$$

whence the expansion of $h(X, Y)$.

(ii) As in Section 1.8.7.d we shall prove that (4.44) and the universality determine the expansions of $A$ and $C$ modulo $Y^{2}$. They are even Lie series, therefore

$$
A(X, Y)=a(x) Y+O\left(Y^{2}\right), C(X, Y)=c(x) Y+O\left(Y^{2}\right)
$$


where $a$ and $c$ are odd power series in the variable $x=\operatorname{ad} X$. Writing (4.44) modulo $Y^{2}$ at first we obtain

$$
Z(Y, X)=X+(x \operatorname{coth} x) Y+O\left(Y^{2}\right)=X+Y-(\operatorname{sh} x) a(x) Y+O\left(Y^{2}\right),
$$

hence $a(x)=(1-x \operatorname{coth} x) / \operatorname{sh} x)$ in view of the next lemma.

Lemma 4.33 Let u be a power series in one variable, convergent in a neighborhood of the origin. The following are equivalent:

(i) for all Lie algebras $u(x) Y=0$ for all $X, Y$ in a neighborhood of 0 in $\mathfrak{g}$ (ii) for all symmetric spaces $u(x) Y=0$ for all $X, Y$ in a neighborhood of 0 in $\mathfrak{s}$

(iii) $u$ is identically 0 .

Proof. Only (ii) $\Rightarrow$ (iii) needs to be proved. For the symmetric space $S L(2, \mathbb{R}) / S O(2), \mathfrak{s}$ is the space of symmetric $2 \times 2$ matrices with trace 0 . Taking

$$
X=\left(\begin{array}{cc}
t / 2 & 0 \\
0 & -t / 2
\end{array}\right), Y=\left(\begin{array}{ll}
0 & 1 \\
1 & 0
\end{array}\right)
$$

we obtain

$$
u(x) Y=\left(\begin{array}{cc}
0 & u(t) \\
u(-t) & 0
\end{array}\right)
$$

and the lemma.

To reach $c(x)$ we need to consider the second order terms in (4.44) as follows. Let $Z(t):=Z(t Y, X)$ and $z(t):=\operatorname{ad} Z(t)$. Then, according to Proposition $4.2(v)$,

$$
Z^{\prime}(t)=\left(\partial_{X} Z\right)(t Y, X) Y=\frac{z(t)}{\operatorname{sh} z(t)} \circ \operatorname{Ad} h(t Y, X) \circ(\operatorname{ch} x) Y .
$$

But $h(t Y, X)=e^{-Z(t)} e^{t Y} e^{X}=e^{Z(t)} e^{-t Y} e^{-X}$ by (4.4), hence

$$
\operatorname{Ad} h(t Y, X) \circ e^{x}=e^{z(t)} e^{-t y}, \operatorname{Ad} h(t Y, X) \circ e^{-x}=e^{-z(t)} e^{t y}
$$

and $\operatorname{Ad} h(t Y, X) \circ(\operatorname{ch} x) Y=(\operatorname{ch} z(t)) Y$, therefore

$$
Z^{\prime}(t)=f(z(t)) Y \text { with } f(z):=z \operatorname{coth} z=1+\sum_{n \geq 1} f_{n} z^{2 n} .
$$

The first order expansion $Z(t)=X+t f(x) Y+O\left(t^{2}\right)$ gives $z(t)=x+$ $t \operatorname{ad}(f(x) Y)+O\left(t^{2}\right)$ whence

$$
\begin{aligned}
Z^{\prime}(t) & =f\left(x+t \operatorname{ad}(f(x) Y)+O\left(t^{2}\right)\right) Y \\
& =f(x) Y+t \sum_{n \geq 1} f_{n} \sum_{1 \leq p \leq 2 n} x^{p-1} \operatorname{ad}(f(x) Y) x^{2 n-p} Y+O\left(t^{2}\right) .
\end{aligned}
$$


A more convenient expression of $Z^{\prime \prime}(0)=\sum_{n \geq 1}(\cdots)$ is obtained by means of Petracci's notation

$$
\left.\left(\zeta\left(x_{1}, x_{2}\right) \mid Y, Y\right)\right):=\sum_{p, q} \zeta_{p q}\left[x^{p} Y, x^{q} Y\right]
$$

where $\zeta(u, v)=\sum_{p, q} \zeta_{p q} u^{p} v^{q}$ is any (commutative) power series. In particular, as in Section 1.8.7.d,

$$
\sum_{1 \leq p \leq 2 n} x^{p-1} \operatorname{ad}(f(x) Y) x^{2 n-p} Y=\left(\frac{\left(x_{1}+x_{2}\right)^{2 n}-x_{2}^{2 n}}{x_{1}} f\left(x_{1}\right) \mid Y, Y\right)
$$

and we infer that $\left.Z^{\prime \prime}(0)=\left(\zeta\left(x_{1}, x_{2}\right) \mid Y, Y\right)\right)$ with

$$
\zeta\left(x_{1}, x_{2}\right)=\frac{f\left(x_{1}+x_{2}\right)-f\left(x_{2}\right)}{x_{1}} f\left(x_{1}\right) .
$$

Thus

$$
\left.Z(t Y, X)=X+t f(x) Y+\frac{t^{2}}{2}\left(\zeta\left(x_{1}, x_{2}\right) \mid Y, Y\right)\right)+O\left(t^{3}\right)
$$

Similarly, let

$$
\left.A(X, t Y)=t a(x) Y+t^{2}\left(\alpha\left(x_{1}, x_{2}\right) \mid Y, Y\right)\right)+O\left(t^{3}\right)
$$

denote the Taylor expansion of $A$ up to order 3 . Transferring this and $C(X, t Y)=t c(x) Y+O\left(t^{2}\right)$ into (4.44) the coefficients of $t^{2}$ lead to the identity $\left.\left(E\left(x_{1}, x_{2}\right) \mid Y, Y\right)\right)=0$, where

$E\left(x_{1}, x_{2}\right):=\frac{1}{2} \zeta\left(x_{1}, x_{2}\right)+\frac{1}{2} x_{2}+a\left(x_{2}\right) \operatorname{ch} x_{2}+c\left(x_{2}\right)+\operatorname{sh}\left(x_{1}+x_{2}\right) \alpha\left(x_{1}, x_{2}\right)$.

Lemma 4.34 Let $E(u, v)$ be a (commutative) power series, convergent in a neighborhood of the origin. The following are equivalent:

(i) for any Lie algebra $\left.\mathfrak{g},\left(E\left(x_{1}, x_{2}\right) \mid Y, Y\right)\right)=0$ identically for $X, Y$ in a neighborhood of the origin of $\mathfrak{g}$

(ii) $E$ is symmetric: $E(u, v)=E(v, u)$.

Proof. Let $E(u, v)=\sum E_{p q} u^{p} v^{q}$ be the given series.

(ii) $\Rightarrow$ (i) clearly, since $\left[x^{p} Y, x^{q} Y\right]$ is skew-symmetric with respect to $(p, q)$.

$(i) \Rightarrow(i i)$. Splitting $E$ as the sum of a symmetric and a skew-symmetric series we may assume $E$ is skew-symmetric and, replacing $X$ by $t X$ in the assumption (i), we infer that $\sum_{p+q=n} E_{p q}\left[x^{p} Y, x^{q} Y\right]=0$ for any $n \geq 1$, or else

$$
\sum_{p+q=n, p>q} E_{p q}\left[x^{p} Y, x^{q} Y\right]=0
$$


for all $X, Y \in \mathfrak{g}$. Let us apply this to the nilpotent Lie algebra of strictly upper triangular $(n+3) \times(n+3)$ real matrices and choose the elements

$$
X=\left(\begin{array}{ccccc}
0 & 1 & \cdots & 0 & 0 \\
& 0 & \ddots & \vdots & \vdots \\
& & \ddots & 1 & 0 \\
& & & 0 & 0 \\
& & & & 0
\end{array}\right), Y=\left(\begin{array}{ccccc}
0 & y_{0} & \cdots & 0 & 0 \\
& 0 & \ddots & \vdots & \vdots \\
& & \ddots & y_{n} & 0 \\
& & & 0 & 1 \\
& & & & 0
\end{array}\right),
$$

which lead to simple computations. Then, for $p+q=n$, the matrix $\left[x^{p} Y, x^{q} Y\right]$ is filled with 0 everywhere except in its upper right corner, equal to

$f_{p}(y)-f_{q}(y)$ with $f_{p}(y)=(-1)^{p} y_{0}+(-1)^{p-1} C_{p}^{1} y_{1}+\cdots-C_{p}^{p-1} y_{p-1}+y_{p}$ (where the $C_{p}^{k}$ 's are the binomial coefficients). Therefore (4.55) implies

$$
\sum_{n / 2<p \leq n} E_{p, n-p}\left(f_{p}(y)-f_{n-p}(y)\right)=0
$$

hence $E_{p, n-p}=0$ since the linear forms $y=\left(y_{0}, \ldots, y_{n}\right) \mapsto f_{p}(y)-f_{n-p}(y)$ are linearly independent. The lemma is proved.

Our previous calculations and Lemma 4.34 imply that

$\frac{f\left(x_{1}+x_{2}\right)-f\left(x_{2}\right)}{2 x_{1}} f\left(x_{1}\right)+\frac{1}{2} x_{2}+a\left(x_{2}\right) \operatorname{ch} x_{2}+c\left(x_{2}\right)+\operatorname{sh}\left(x_{1}+x_{2}\right) \alpha\left(x_{1}, x_{2}\right)$

is a symmetric function of $\left(x_{1}, x_{2}\right)$. In particular, taking $x_{1}=-x, x_{2}=x$, we see that

$$
\frac{f(x)-1}{2 x} f(-x)+\frac{x}{2}+a(x) \operatorname{ch} x+c(x)
$$

is an even function of $x$, thus identically 0 since $f$ is even and $a, c$ are odd. We finally obtain

$$
c(x)=\frac{1-f(x)}{2 x} f(x)-\frac{x}{2}-a(x) \operatorname{ch} x=\frac{x-\operatorname{sh} x \operatorname{ch} x}{2 \operatorname{sh}^{2} x},
$$

which completes the proof of Lemma 4.32 .

\subsubsection{Expansion of $\Phi$ Modulo $Y^{2}$}

Lemma 4.35 Let $\Phi$ be constructed from a solution $(A, C)$ of (4.44). Then

$$
\begin{aligned}
\Phi(X, Y) & =\left(X+\left(1-\frac{x}{\operatorname{sh} x}\right) Y+O\left(Y^{2}\right), Y+O\left(Y^{2}\right)\right) \\
h \circ \Phi(X, Y) & =\exp \left(\left(\operatorname{th} \frac{x}{2}\right) Y+O\left(Y^{2}\right)\right) .
\end{aligned}
$$


Proof. We refer to 4.2 .4 for the definition and properties of $\Phi_{t}(X, Y)=$ $\left(X_{t}, Y_{t}\right)=t^{-1} \Phi(t X, t Y)$; in particular $\Phi_{t}(X, 0)=(X, 0)$ (Theorem $4.6($ iii $\left.)\right)$. Let us recall (4.13):

$$
\partial_{t} Y_{t}(X, Y)=\left[B_{t} \circ \Phi_{t}(X, Y), Y_{t}(X, Y)\right] .
$$

Taking derivatives with respect to a component $Y_{j}$ of $Y$ (with respect to some basis of $\mathfrak{s})$ we obtain $\partial_{t}\left(\partial_{Y_{j}} Y_{t}\right)(X, 0)=0$ since $B_{t} \circ \Phi_{t}(X, 0)=B_{t}(X, 0)=0$ and $Y_{t}(X, 0)=0$. Thus $\partial_{Y} Y_{t}(X, 0)$ does not depend on $t$. Since $Y_{0}(X, Y)=Y$ it is the identity, and the second component of $\Phi(X, Y)$ is $Y+O\left(Y^{2}\right)$.

Let $\left(X_{1}, Y_{1}\right)=\Phi(X, Y)$. We have $Z\left(X_{1}, Y_{1}\right)=X+Y$ by the fundamental property of $\Phi$ therefore, by differentiation with respect to $Y$ at $Y=0$,

$$
\partial_{X} Z(X, 0) \circ \partial_{Y} X_{1}(X, 0)+\partial_{Y} Z(X, 0) \circ \partial_{Y} Y_{1}(X, 0)=1,
$$

the identity mapping of $\mathfrak{s}$. The derivatives of $Z$ are given by Proposition 4.2 $(v)$, with $Z=X$ and $h=e$ for $Y=0$, and $\partial_{Y} Y_{1}(X, 0)=1$ by the first part of the proof. It follows that

$$
\partial_{Y} X_{1}(X, 0)=1-\frac{x}{\operatorname{sh} x},
$$

whence our claim for the first component of $\Phi$.

Then $h \circ \Phi(X, Y)=h\left(X+O(Y), Y+O\left(Y^{2}\right)\right)$ and Lemma $4.32(i)$ implies (4.57).

Remark. For $\left(X_{t}, Y_{t}\right)=t^{-1} \Phi(t X, t Y)$ the lemma gives

$$
X_{t}=X+\left(1-\frac{t x}{\operatorname{sh} t x}\right) Y+O\left(Y^{2}\right), Y_{t}=Y+O\left(Y^{2}\right),
$$

whence a new proof of the expansion (4.52) of $A$. Indeed, writing $A(X, Y)=$ $a(x) Y+O\left(Y^{2}\right)$, the first order terms with respect to $Y$ in the differential equation $\partial_{t} X_{t}=\left[A_{t}\left(X_{t}, Y_{t}\right), X_{t}\right]$ lead to

$$
\partial_{t}\left(1-\frac{t x}{\operatorname{sh} t x}\right) Y=-x a(t x) Y
$$

hence, for $t=1$,

$$
\left(\frac{1-x \operatorname{coth} x}{\operatorname{sh} x}-a(x)\right) x Y=0
$$

and the result by the universality of $A$ (Lemma 4.33). Thus $A$ is uniquely determined modulo $Y^{2}$ by its universality and the properties of $\Phi$.

\subsubsection{Expansions of $e$ and $e_{\chi}$ Modulo $Y^{2}$}

For a line bundle $L_{\chi}$ we recall the notation $\chi^{\prime}$ (the differential of the character $\chi$ at the origin), replaced by $\omega^{\prime}=\frac{1}{2} \operatorname{tr}_{\mathfrak{h}}$ ad for the bundle of half-densities. 
Proposition 4.36 Let $e$ be the $\left(J^{1 / 2}, \Phi\right)$-function, where $\Phi$ is constructed from even Lie series $A, C$ which are universal solutions of (4.44). Then, for $X, Y$ near the origin of $\mathfrak{s}$,

$$
\log e(X, Y)=\frac{1}{2} \operatorname{tr}_{\mathfrak{h}} \text { ad }\left(\left(\operatorname{th} \frac{x}{2}\right) Y\right)+O\left(Y^{2}\right)=\frac{1}{4} \operatorname{tr}_{\mathfrak{h}}[\operatorname{th} x, y]+O\left(Y^{2}\right) .
$$

More generally, for any character $\chi$ of $H$,

$$
\log e_{\chi}(X, Y)=\left(\omega^{\prime}-\chi^{\prime}\right)\left(\left(\operatorname{th} \frac{x}{2}\right) Y\right)+O\left(Y^{2}\right) .
$$

Proof. By (4.32) and (4.35) in Proposition $4.19(i)$ we have, with $t=1$ for simplicity and $f(x):=x \operatorname{coth} x$,

$$
\begin{aligned}
& \left.2 \partial_{t} \log e_{t}(X, Y)\right|_{t=1}= \\
& =\operatorname{tr}_{\mathfrak{h}}(f(x)-f(x+y)+f(y)-1)-\operatorname{tr}_{\mathfrak{h}}\left(2 \partial_{Y} C \circ y+2 \operatorname{ad} A\right) \circ \Phi(X, Y) .
\end{aligned}
$$

We know from Lemmas 4.32 and 4.35 that

$$
\begin{aligned}
& C(X, Y)=c(x) Y+O\left(Y^{2}\right), A(X, Y)=a(x) Y+O\left(Y^{2}\right) \\
& \Phi(X, Y)=\left(X+O(Y), Y+O\left(Y^{2}\right)\right)
\end{aligned}
$$

with $2 c(x)=(x-\operatorname{sh} x \operatorname{ch} x) / \operatorname{sh}^{2} x=-f^{\prime}(x)$ and $a(x)=(1-x \operatorname{coth} x) / \operatorname{sh} x$. Since $f(y)-1=O\left(Y^{2}\right)$ we infer

$$
\begin{aligned}
& \left.2 \partial_{t} \log e_{t}(X, Y)\right|_{t=1}= \\
& \quad=\operatorname{tr}_{\mathfrak{h}}(f(x)-f(x+y))-\operatorname{tr}_{\mathfrak{h}}\left(-f^{\prime}(x) y+2 \operatorname{ad}(a(x) Y)\right)+O\left(Y^{2}\right) .
\end{aligned}
$$

Lemma 4.37 (i) For any even power series $f$ in one variable

$$
\operatorname{tr}_{\mathfrak{h}}(f(x+y)-f(x))=\frac{1}{2} \operatorname{tr}_{\mathfrak{h}}\left(f^{\prime}(x) y+y f^{\prime}(x)\right)+O\left(Y^{2}\right) .
$$

(ii) For any odd power series a in one variable

$$
2 \operatorname{tr}_{\mathfrak{h}} \operatorname{ad}(a(x) Y)=\operatorname{tr}_{\mathfrak{h}}[a(2 x), y] .
$$

Proof. (i) is easily checked for $f(x)=x^{2 n}$.

(ii) Since $\operatorname{ad}\left(x^{n+1} Y\right)=\left[x, \operatorname{ad}\left(x^{n} Y\right)\right]$ it follows that

$$
\operatorname{ad}\left(x^{n} Y\right)=\sum_{k=0}^{n}(-1)^{k} C_{n}^{k} x^{n-k} y x^{k}
$$

inductively, where $C_{n}^{k}$ is the binomial coefficient. Replacing $n$ by $2 n+1$ we can separate out even and odd $k$ 's so as to obtain, after repeated commutation of $x^{2}$ with even monomials,

$$
\operatorname{ad}\left(x^{2 n+1} Y\right)=\left(\sum_{k} C_{2 n+1}^{2 k}\right) x^{2 n+1} y-\left(\sum_{k} C_{2 n+1}^{2 k+1}\right) y x^{2 n+1}+\left[x^{2}, v_{2 n}\right]
$$


where $v_{2 n}$ is an even monomial in $(x, y)$. Thus

$$
2 \operatorname{ad}\left(x^{2 n+1} Y\right)=\left[(2 x)^{2 n+1}, y\right]+2\left[x^{2}, v_{2 n}\right]
$$

and the lemma follows.

Going back to the proof of Proposition 4.36 we now obtain by elementary computations

$$
\begin{aligned}
\left.\partial_{t} \log e_{t}(X, Y)\right|_{t=1} & =\frac{1}{4} \operatorname{tr}_{\mathfrak{h}}\left(\left[f^{\prime}(x), y\right]-2[a(2 x), y]\right)+O\left(Y^{2}\right) \\
& =\frac{1}{4} \operatorname{tr}_{\mathfrak{h}}\left[\operatorname{th} x+\frac{x}{\operatorname{ch}^{2} x}, y\right]+O\left(Y^{2}\right) .
\end{aligned}
$$

Then

$$
\begin{aligned}
\left(\partial_{t} \log e_{t}\right)(X, Y) & =t^{-1}\left(\left.\partial_{s} \log e_{s}\right|_{s=1}\right)(t X, t Y) \\
& =\frac{1}{4} \operatorname{tr} \mathfrak{h}\left[\operatorname{th} t x+\frac{t x}{\operatorname{ch}^{2} t x}, y\right]+O\left(Y^{2}\right) \\
& =\frac{1}{4} \operatorname{tr}_{\mathfrak{h}}\left[\partial_{t}(t \operatorname{th} t x), y\right]+O\left(Y^{2}\right)
\end{aligned}
$$

and integration from $t=0$ to $t=1$ gives

$$
\log e(X, Y)=\frac{1}{4} \operatorname{tr}_{\mathfrak{h}}[\operatorname{th} x, y]+O\left(Y^{2}\right) .
$$

The other expression of $\log e$ follows from (ii) in the lemma.

For the line bundle

$$
\begin{aligned}
\log e_{\chi} & =\log e-\chi^{\prime}(\log (h \circ \Phi))=\log e-\chi^{\prime}\left(\left(\operatorname{th} \frac{x}{2}\right) Y\right)+O\left(Y^{2}\right) \\
& =\left(\omega^{\prime}-\chi^{\prime}\right)\left(\left(\operatorname{th} \frac{x}{2}\right) Y\right)+O\left(Y^{2}\right)
\end{aligned}
$$

by (4.57) and the proof is complete.

\subsection{Open Problems}

a. Characterize special symmetric spaces in a more explicit way than Theorem 4.22 (iii). Give other examples (see $\mathbf{c}$ below).

b. Characterize the line bundles $L_{\chi}$ such that $\mathbb{D}\left(L_{\chi}\right)$ is a commutative algebra (see Remarks after Theorem 4.28).

c. Besides solvable and strongly symmetric spaces (Theorems 4.22 and 4.27), another interesting candidate for a special line bundle was introduced by 
Alekseev and Meinrenken in [4]. These authors consider a quadratic symmetric algebra $(\mathfrak{g}, \sigma)$ with skew $\sigma$-invariant quadratic form, that is a nondegenerate symmetric bilinear form $B$ such that $B([X, Y], Z)=-B(X,[Y, Z])$ and $B(\sigma X, \sigma Y)=-B(X, Y)$ for all $X, Y, Z \in \mathfrak{g}$; examples are given in Section 8.2 of [4]. Using their non-commutative Chern-Weil homomorphism, they prove a version of Duflo's isomorphism in this case: our transfer map $\sim$ is an isomorphism of the algebra of $H$-invariant differential operators with constant coefficients on $\mathfrak{s}$ onto the algebra of $G$-invariant differential operators on the bundle of half-densities on $G / H$. A different proof (and a generalization) was given by Torossian [56]. It is therefore natural to conjecture that our function $e_{\omega}$ is identically 1 in this case.

Yet another proof of the same result appears in Section 3.6 of the paper ${ }^{5}$ [15] by Cattaneo and Torossian who, more generally, propose a completely different approach to $e$-functions. Using Kontsevich diagrams they construct a function $E_{\chi}(X, Y)$ for the line bundle $L_{\chi}$, which has many similarities to our $e_{\omega \chi^{-1}}(X, Y)$. It would be interesting to know whether both functions coincide.

d. Can the $\left(J^{1 / 2}, F, G\right)$-function of 4.4.2 be written as

$$
\log e(X, Y)=\operatorname{tr}_{\mathfrak{h}} \operatorname{ad}(a(x, y) T)+\left(B_{\mathfrak{g}}-2 B_{\mathfrak{h}}\right)(T, b(x, y) T)
$$

where $T=[X, Y]$ and $a(x, y), b(x, y)$ are even non-commutative series? This result (suggested by the expansion in Proposition 4.31) would improve Theorem $4.20(i)$ : since $a(x, y) T=[X, V]+[Y, W]$ for some $V, W \in \mathfrak{s}$ and $b(x, y) T=T^{\prime}$ belongs to $\mathfrak{h}$, the above expression would imply

$$
\begin{aligned}
\log e & =\operatorname{tr}_{\mathfrak{h}}([x, v]+[y, w])+\left(\operatorname{tr}_{\mathfrak{s}}-\operatorname{tr}_{\mathfrak{h}}\right)\left((x y-y x) t^{\prime}\right) \\
& =\operatorname{tr}_{\mathfrak{h}}\left(\left[x, v-t^{\prime} y\right]+\left[y, w+t^{\prime} x\right]\right)
\end{aligned}
$$

with $v=\operatorname{ad} V, w=\operatorname{ad} W, t^{\prime}=\operatorname{ad} T^{\prime}$. It would also give a direct proof that strongly symmetric spaces are special (see Remark (b) after Theorem 4.22).

e. The property $e(X, Y)=e(X, Z)$ whenever $X+Y+Z=0$ holds true for the rank one function of Section 3.7 (Proposition 3.24 (ii)) and obviously for special spaces. Is is true in some other cases?

\section{Notes}

This chapter collects several facts on $e$-functions directly related to their construction by means of the Campbell-Hausdorff formula, in the spirit of the fundamental work [30]. A large part of the chapter is taken from my papers [43] - [47]. Most proofs have been rewritten however, because their counterparts in those papers made use of a specific choice of the Lie series

\footnotetext{
${ }^{5}$ See also the correction in [14].
} 
$F, G$, given by some integrals which we do not need any more. Theorem 4.16 is new. The function $c(X, Y)$ was introduced in [46]. Section 4.4, which contains our main theorems, is partly new, with some results relying on the Kashiwara-Vergne conjecture now proved in full generality (see Chapter 1). The proof of Lemma 4.32 is adapted from Alekseev and Petracci [6] (arXiv version).

In [55] Torossian gives a new proof of Theorem 4.22 by means of the Kontsevich star product adapted to symmetric spaces.

Theorem 4.28 implies the commutativity of the algebra $\mathbb{D}\left(L_{\chi}\right)$ if $\chi^{\prime}-\omega^{\prime}$ extends to a character of $\mathfrak{g}$. It gives a new proof of a result due to Michel Duflo [19], generalizing a previous theorem by André Lichnerowicz [36] which states that $\mathbb{D}(S)$ is commutative if $S$ is a symmetric space with $G$-invariant measure. 


\section{Appendix A}

\section{Proof of Lemma 3.22}

Keeping to the semisimple notation in use in Section 3.6, we recall that the radial component of $g \in G$ is defined as the unique $R(g) \in \overline{\mathfrak{a}^{+}}$such that

$$
g=k e^{R(g)} k^{\prime}
$$

for some $k, k^{\prime} \in K$ (Cartan decomposition in $G$, see [27] p. 402). Let $|X|=$ $\sqrt{\langle X, X\rangle}$ denote the norm on $\mathfrak{g}, o$ the origin of $G / K$ and $d(.,$.$) its distance$ function.

Lemma A.1 For all $g, g^{\prime} \in G$

$$
\left|R(g)-R\left(g^{\prime}\right)\right| \leq d\left(g \cdot o, g^{\prime} \cdot o\right) .
$$

Remark. This inequality is equivalent to $d\left(a \cdot o, a^{\prime} \cdot o\right) \leq d\left(k a \cdot o ; a^{\prime} \cdot o\right)$ for all $a, a^{\prime} \in \overline{\operatorname{exp\mathfrak {a}^{+}}}$ and $k \in K$.

Proof. ${ }^{1}$ The classical decomposition $G=(\exp \mathfrak{p}) K$ reduces the problem to the case $g=e^{X}, g^{\prime}=e^{X^{\prime}}$ with $X, X^{\prime} \in \mathfrak{p}$. We split the proof into two steps. (i) $\left|R\left(e^{X}\right)-R\left(e^{X^{\prime}}\right)\right| \leq\left|X-X^{\prime}\right|$.

Indeed, by Cartan decomposition in $\mathfrak{p}$ we may write $X=k \cdot H, X^{\prime}=k^{\prime} \cdot H^{\prime}$ for some $k, k^{\prime} \in K, H, H^{\prime} \in \overline{\mathfrak{a}^{+}}$and Proposition 5.18 in [28] p. 196 implies

$$
\left|R\left(e^{X}\right)-R\left(e^{X^{\prime}}\right)\right|=\left|H-H^{\prime}\right| \leq\left|k \cdot H-k^{\prime} \cdot H^{\prime}\right|=\left|X-X^{\prime}\right| .
$$

(ii) $\left|X-X^{\prime}\right| \leq d\left(\operatorname{Exp} X, \operatorname{Exp} X^{\prime}\right)=d\left(e^{X} \cdot o, e^{X^{\prime}} \cdot o\right)$.

Indeed $d\left(\operatorname{Exp} X, \operatorname{Exp} X^{\prime}\right)$ is the length of the geodesic segment $\Gamma$ joining both points. Let $\gamma$ be the corresponding curve segment in the chart Exp, joining $X$ to $X^{\prime}$ in $\mathfrak{p}$. Then ( $\ell$ denoting length) $\left|X-X^{\prime}\right| \leq \ell(\gamma)$ in the Euclidean space $\mathfrak{p}$. Besides $\ell(\gamma) \leq \ell(\Gamma)$ by a general property, due to Élie Cartan, of

\footnotetext{
${ }^{1}$ I am indebted to Sigurður Helgason for shortening my original proof of the lemma.
} 
the exponential chart in a Riemannian manifold of negative curvature ([27] p. 73). Thus $\left|X-X^{\prime}\right| \leq \ell(\Gamma)$ and the proof is complete.

Remark. For $G / K$ Cartan's theorem is easily proved as follows. The point is to show that $\left|\left(D_{X} \operatorname{Exp}\right) V\right| \geq|V|$ for $X, V \in \mathfrak{p}$, with $D_{X} \operatorname{Exp}=$ $D_{o} \tau\left(e^{X}\right) \circ(\operatorname{sh} a d X / \operatorname{ad} X)$. By Cartan decomposition of $X$ it suffices to consider $X \in \mathfrak{a}$. In the orthogonal decomposition $\mathfrak{p}=\mathfrak{a} \oplus\left(\oplus_{\alpha>0} \mathfrak{p}_{\alpha}\right)$ the endomorphism $(\operatorname{ad} X)^{2}$ is then diagonal with respective eigenvalues $0, \alpha(X)^{2}$, therefore sh ad $X / \operatorname{ad} X=1+\sum_{1}^{\infty}(\operatorname{ad} X)^{2 n} /(2 n+1)$ ! is diagonal with eigenvalues $\geq 1$ and the result follows.

Proof of Lemma 3.22. We now explain how Lemma A.1 implies the estimate

$$
R\left(e^{X} e^{t H}\right)=t H+A(X)+O\left(e^{-t \mu(H)}\right)
$$

as $t \rightarrow+\infty$ for $X \in \mathfrak{p}, H \in \mathfrak{a}^{+}$, with $\mu(H):=\inf _{\alpha>0} \alpha(H)$.

First, replacing $g$ by $e^{H^{\prime}} g$ and $g^{\prime}$ by $e^{H^{\prime}}$ with $H^{\prime} \in \overline{\mathfrak{a}^{+}}$, the lemma gives

$$
\left|R\left(e^{H^{\prime}} g\right)-H^{\prime}\right| \leq d\left(e^{H^{\prime}} g \cdot o, e^{H^{\prime}} \cdot o\right)=d(o, g \cdot o) .
$$

Then let $e^{X}=k(X) e^{A(X)} e^{\Sigma X_{\alpha}}$ be the Iwasawa decomposition for $X \in \mathfrak{p}$, with $k(X) \in K, A(X) \in \mathfrak{a}, X_{\alpha} \in \mathfrak{g}_{\alpha}$, the sum $\Sigma$ running over all positive roots $\alpha$. Fixing $H \in \mathfrak{a}^{+}$and remembering $\left[H, X_{\alpha}\right]=\alpha(H) X_{\alpha}$ we obtain

$$
e^{X} e^{t H}=k(X) e^{t H+A(X)} e^{V(t)} \text { with } V(t):=\sum_{\alpha>0} e^{-t \alpha(H)} X_{\alpha} \in \mathfrak{n} .
$$

Since $\alpha(H)>0$ for all positive roots $\alpha$, we have $t H+A(X) \in \mathfrak{a}^{+}$for $t$ large enough (uniformly for $X$ in a compact subset of $\mathfrak{p}$ ) and (A.1) applies to $R\left(e^{t H+A(X)} e^{V(t)}\right)=R\left(e^{X} e^{t H}\right)$ :

$$
\left|R\left(e^{X} e^{t H}\right)-t H-A(X)\right| \leq d\left(o, e^{V(t)} \cdot o\right) .
$$

The latter distance can be evaluated by means of the decomposition $G=$ $(\exp \mathfrak{p}) K$. Forgetting $t$ for the moment let us write $e^{s V}=e^{W(s)} k(s)$ with $W(s) \in \mathfrak{p}$ and $k(s) \in K$, smooth functions of $s \in \mathbb{R}$, hence $e^{2 W(s)}=e^{s V} e^{-s \theta V}$ and $d\left(o, e^{V} \cdot o\right)=|W(1)|$. Disregarding a trivial case we assume $V \neq 0$. For the $s$-derivative $W^{\prime}$ we obtain, with $w=\operatorname{ad} W(s)$,

$$
\frac{1-e^{-2 w}}{2 w} 2 W^{\prime}=\left(e^{-2 w}-1\right) V+(V-\theta V) .
$$

Since $\left(1-e^{-2 w}\right) / 2 w=e^{-w}(\operatorname{sh} w) / w$ is, for $W \in \mathfrak{p}$, an invertible endomorphism of $\mathfrak{p}$ it follows that $2 W^{\prime}=V-\theta V+[W, \cdots]$ and, by scalar product with $W$,

$$
2\left\langle W(s), W^{\prime}(s)\right\rangle=\langle W(s), V-\theta V\rangle,
$$


hence $f^{\prime}(s) \leq|V-\theta V| \sqrt{f(s)}$ with $f(s)=|W(s)|^{2}$. To integrate this, observe that $f(0)=0$ and $f(s)>0$ for $s \neq 0$ because $W(s)=0$ implies $e^{s V}=e^{s \theta V} \in N \cap \theta N=\{e\}$ hence $s=0$. Thus $\sqrt{f(s)} \leq \frac{s}{2}|V-\theta V|$ and

$$
d\left(o, e^{V} \cdot o\right)=\sqrt{f(1)} \leq \frac{1}{2}|V-\theta V|
$$

For $V=V(t)$ defined above we have $|V-\theta V| \leq C e^{-t \mu(H)}$ with $\mu(H)=$ $\inf _{\alpha>0} \alpha(H)>0$ and a constant $C$ uniform for all $X$ in a compact subset of $\mathfrak{p}$. This completes the proof of Lemma 3.22. 


\section{Appendix B}

\section{Proof of Theorem 3.23}

Here $G / K$ is a rank one Riemannian symmetric space of the noncompact type and we use the notation of Section 3.7. As explained in the outline 3.7.1 our main task is to make explicit the kernels $a, b$ in the integral formulas (Lemmas B.2 and B.5 below)

$$
\begin{aligned}
\int_{K} \varphi(\|x H+k \cdot y H\|) d k & =\int_{|x-y|}^{x+y} \varphi(z) a(x, y, z) d z \\
\int_{K} \varphi(\|Z(x H, k \cdot y H)\|) d k & =\int_{|x-y|}^{x+y} \varphi(z) b(x, y, z) d z
\end{aligned}
$$

where $\varphi$ is a continuous function on $[0, \infty[, H \in \mathfrak{a}$ with $\alpha(H)=1,\|H\|=1$ and $x, y>0$. As usual the Haar measure $d k$ over $K$ is normalized by $\int_{K} d k=$ 1. After identification of $\mathfrak{a}=\mathbb{R} H$ with $\mathbb{R}$ the orthogonal projection $\pi: \mathfrak{p} \rightarrow \mathfrak{a}$ is $\pi(X)=X \cdot H$ (the dot denotes here the scalar product on $\mathfrak{p}$ corresponding to the norm $\|\cdot\|$ in $(3.44))$.

Lemma B.1 Let $f$ be a continuous function on $[-1,1]$. Then

$$
\int_{K} f(\pi(k \cdot H)) d k=\frac{\Gamma\left(\frac{n}{2}\right)}{\Gamma\left(\frac{n-1}{2}\right) \Gamma\left(\frac{1}{2}\right)} \int_{-1}^{1} f(t)\left(1-t^{2}\right)^{(n-3) / 2} d t .
$$

Proof. The map $k \mapsto k \cdot H=\operatorname{Ad}(k) H$ induces a diffeomorphism of $K / M$ onto the unit sphere $\Sigma$ of $\mathfrak{p}$. The classical spherical measure $d \sigma$ on $\Sigma$ is invariant under all isometries preserving the origin of $\mathfrak{p}$, therefore under the adjoint action of $K$, and $d \sigma$ corresponds to a $K$-invariant measure on $K / M$ : there exists a positive constant $C$ such that

$$
\int_{K} f(\pi(k \cdot H)) d k=\int_{K / M} f(\pi(k \cdot H)) d(k M)=C \int_{\Sigma} f\left(x_{1}\right) d \sigma(X) .
$$

Here $X=\left(x_{1}, \ldots, x_{n}\right)$ are coordinates with respect to an orthonormal basis of $\mathfrak{p}$, with $H$ as the first basis vector. Given $t \in[-1,1]$ the intersection of $\Sigma$ 
with the hyperplane $x_{1}=t$ is (for $\left.n \geq 3\right)$ a $(n-2)$-dimensional sphere with radius $\sqrt{1-t^{2}}$ and it follows that (with another constant $C^{\prime}$ )

$$
\int_{K} f(\pi(k \cdot H)) d k=C^{\prime} \int_{-1}^{1} f(t)\left(1-t^{2}\right)^{(n-3) / 2} d t,
$$

which remains valid for $n=2$ too. Taking $f=1$ we obtain the value of $C^{\prime}$ and the lemma.

Lemma B.2 Given $x, y>0$ let $\varphi$ be continuous on $[|x-y|, x+y]$. Then, for all rank one spaces,

$$
\int_{K} \varphi(\|x H+k \cdot y H\|) d k=\int_{|x-y|}^{x+y} \varphi(z) a(x, y, z) d z
$$

with

$$
\begin{aligned}
a(x, y, z)= & \frac{2^{3-n} \Gamma\left(\frac{n}{2}\right)}{\Gamma\left(\frac{n-1}{2}\right) \Gamma\left(\frac{1}{2}\right)} \times \\
& \times z \frac{((x+y+z)(x+y-z)(x-y+z)(-x+y+z))^{(n-3) / 2}}{(x y)^{n-2}}
\end{aligned}
$$

One has $a(x, y, z)>0$ for $x, y>0$ and $|x-y|<z<x+y$.

Proof. Since $\|x H+k \cdot y H\|^{2}=x^{2}+y^{2}+2 x y \pi(k \cdot H)$, this follows from the previous lemma with $f(t)=\varphi\left(\sqrt{x^{2}+y^{2}+2 x y t}\right)$ and the change of variable $t \mapsto z=\sqrt{x^{2}+y^{2}+2 x y t}$.

Remark. A similar proof would give John's formula for the iterated spherical means (see [29] p. 356), where the same factor $a(x, y, z)$ appears.

Lemma B.1 turns out to imply (B.2) too in the simple case of real hyperbolic spaces, as follows.

Lemma B.3 Given $x, y>0$ let $\varphi$ be continuous on $[|x-y|, x+y]$. Then, for $H^{n}(\mathbb{R})$

$$
\int_{K} \varphi(\|Z(x H, k \cdot y H)\|) d k=\int_{|x-y|}^{x+y} \varphi(z) b(x, y, z) d z
$$

with

$$
b(x, y, z)=\frac{2^{n-3} \Gamma\left(\frac{n}{2}\right)}{\Gamma\left(\frac{n-1}{2}\right) \Gamma\left(\frac{1}{2}\right)} \frac{(\operatorname{ch} x \operatorname{ch} y \operatorname{ch} z)^{(n-3) / 2}}{(\operatorname{sh} x \operatorname{sh} y)^{n-2}} \operatorname{sh} z B^{(n-3) / 2}
$$

and

$$
\begin{aligned}
B= & \frac{1}{\operatorname{ch} x \operatorname{ch} y \operatorname{ch} z} \times \\
& \times \operatorname{sh}\left(\frac{x+y+z}{2}\right) \operatorname{sh}\left(\frac{x+y-z}{2}\right) \operatorname{sh}\left(\frac{x-y+z}{2}\right) \operatorname{sh}\left(\frac{-x+y+z}{2}\right)
\end{aligned}
$$


Proof. Here $H^{n}(\mathbb{R})=G / K$ with $G=S O_{0}(n, 1), K=S O(n) \times\{1\}$, and $\mathfrak{p}$ is the set of matrices

$$
Y=\left(\begin{array}{cccc}
0 & 0 & 0 & y_{1} \\
0 & 0 & 0 & \vdots \\
0 & 0 & 0 & y_{n} \\
y_{1} & \cdots & y_{n} & 0
\end{array}\right)
$$

identified with $Y=\left(y_{1}, \ldots, y_{n}\right) \in \mathbb{R}^{n}$ for short. We take the unit vector $H=(1,0, \ldots, 0)$ as a basis of $\mathfrak{a}$. The adjoint action of $K$ on $\mathfrak{p}$ is the natural action of $S O(n)$ on $\mathbb{R}^{n}$. Since

$$
e^{Y}=I+\frac{\operatorname{sh} y}{y} Y+\frac{\operatorname{ch} y-1}{y^{2}} Y^{2}
$$

with $y=\|Y\|=\left(\sum_{1}^{n} y_{i}^{2}\right)^{1 / 2}$, the equality $e^{Z} K=e^{x H} e^{Y} K$ implies (looking at the element in the last row and column) $\operatorname{ch} z=\operatorname{ch} x \operatorname{ch} y+\operatorname{sh} x \frac{\operatorname{sh} y}{y} y_{1}$ with $z=\|Z\|$, hence $z \in[|x-y|, x+y]$. Taking $Y=k \cdot y H$ with $y \in \mathbb{R}$ and $k \in K$ we see that $z=\|Z(x H, k \cdot y H)\|$ is given by

$$
\operatorname{ch} z=\operatorname{ch} x \operatorname{ch} y+\pi(k \cdot H) \operatorname{sh} x \operatorname{sh} y .
$$

Then, by Lemma B.1,

$$
\int_{K} \varphi(z) d k=\frac{\Gamma\left(\frac{n}{2}\right)}{\Gamma\left(\frac{n-1}{2}\right) \Gamma\left(\frac{1}{2}\right)} \int_{-1}^{1} f(t)\left(1-t^{2}\right)^{(n-3) / 2} d t
$$

if $\varphi$ and $f$ are related by $\varphi(z)=f(t)$ and $\operatorname{ch} z=\operatorname{ch} x \operatorname{ch} y+t \operatorname{sh} x \operatorname{sh} y$. Expressing the latter integral with the variable $z$, the result now follows since

$$
1-t^{2}=4 \frac{\operatorname{ch} x \operatorname{ch} y \operatorname{ch} z}{\operatorname{sh}^{2} x \operatorname{sh}^{2} y} B(x, y, z) .
$$

Our goal is now to prove Lemma B.5, giving (B.2) for the other hyperbolic spaces, by the classical technique of reduction to $S U(2,1)$ ([27], Chapter IX, $\S 3)$. Let $V \in \mathfrak{g}_{-\alpha}$ and $W \in \mathfrak{g}_{-2 \alpha}$ be fixed, with $\|V\|=\|W\|=\sqrt{2}$. The next lemma reduces integration of $M$-bi-invariant functions on $K$ to a 2dimensional integral; for a similar lemma with different coordinates see Orloff [39] p. 588. Let us recall that $\operatorname{dim} G / K=n=p+q+1$ with $p=\operatorname{dim} \mathfrak{g}_{-\alpha}$, $q=\operatorname{dim} \mathfrak{g}_{-2 \alpha}$ and let $g=k(g) e^{H(g)} n(g)$ denote the Iwasawa decomposition of $g \in G$.

Lemma B.4 Let $k(r, \omega):=k(\bar{n})$ be the K-component of $\bar{n}=\exp (v V+w W)$ $\in \bar{N}=\theta N$, where $v>0, w>0$ are related to $(r, \omega)$ by

$$
1+r e^{i \omega}=\frac{2}{1+v^{2}-2 i w} .
$$


Then, for any continuous function $f$ on $K$ such that $f\left(m k m^{\prime}\right)=f(k)$ for $m, m^{\prime} \in M$ and $k \in K$,

(i) if $q>1$ (quaternionic and exceptional hyperbolic spaces)

$$
\begin{aligned}
& \int_{K} f(k) d k= \\
& \quad=\frac{2 \Gamma\left(\frac{n}{2}\right)}{\Gamma\left(\frac{p}{2}\right) \Gamma\left(\frac{q}{2}\right) \Gamma\left(\frac{1}{2}\right)} \int_{0}^{1}\left(1-r^{2}\right)^{(p / 2)-1} r^{q} d r \int_{0}^{\pi} f(k(r, \omega)) \sin ^{q-1} \omega d \omega
\end{aligned}
$$

(ii) if $q=1$ (complex hyperbolic spaces)

$$
\int_{K} f(k) d k=\frac{p}{2 \pi} \int_{0}^{1}\left(1-r^{2}\right)^{(p / 2)-1} r d r \int_{-\pi}^{\pi} f(k(r, \omega)) d \omega .
$$

Proof. (i) The $M$-invariance of $f$ implies

$$
\int_{K} f(k) d k=\int_{\bar{N}} f(k(\bar{n})) e^{-2<\rho, H(\bar{n})>} d \bar{n}
$$

by a classical integral formula valid for arbitrary rank ([28] p. 198), if the Haar measure $d \bar{n}$ is suitably normalized. In the rank one case $\bar{N}=$ $\exp \left(\mathfrak{g}_{-\alpha} \oplus \mathfrak{g}_{-2 \alpha}\right)$; if $q>1$ we may use polar coordinates $(v, \sigma)$ in $\mathfrak{g}_{-\alpha}$, resp. $(w, \tau)$ in $\mathfrak{g}_{-2 \alpha}$, and obtain

$$
\begin{aligned}
& \int_{K} f(k) d k= \\
& \quad=C \int f(k(\exp (v \sigma+w \tau))) e^{-2<\rho, H(\exp (v \sigma+w \tau))>} v^{p-1} w^{q-1} d v d w d \sigma d \tau
\end{aligned}
$$

where $C$ is a constant, $v, w$ run over $] 0, \infty[$ and $\sigma, \tau$ over the unit spheres $S_{1}, S_{2}$ of $\mathfrak{g}_{-\alpha}, \mathfrak{g}_{-2 \alpha}$ with measures $d \sigma, d \tau$. By a theorem of Kostant ([60] p. 265) Ad $M$ acts transitively on $S_{1} \times S_{2}$ if $q>1$ so that, in view of $\|V\|=\|W\|=\sqrt{2}$, we have $v \sigma+w \tau=m \cdot\left(\frac{v}{\sqrt{2}} V+\frac{w}{\sqrt{2}} W\right)$ for some $m \in M$.

But $M$ commutes with $A$ and normalizes $N$, therefore

$$
k\left(m \bar{n} m^{-1}\right)=m k(\bar{n}) m^{-1}, H\left(m \bar{n} m^{-1}\right)=H(\bar{n})
$$

for $m \in M, \bar{n} \in \bar{N}$ and, by the $M$-invariance of $f$,

$$
\begin{aligned}
& \int_{K} f(k) d k= \\
= & C^{\prime} \int f(k(\exp (v V+w W))) e^{-2<\rho, H(\exp (v V+w W))>} v^{p-1} w^{q-1} d v d w
\end{aligned}
$$

an integral over $v>0, w>0$ with another constant $C^{\prime}$. 
The relation $\left(1+r e^{i \omega}\right)\left(1+v^{2}-2 i w\right)=2$ defines a change of variables $(v, w) \mapsto(r, \omega)$ (which will be convenient to prove the next lemma), a diffeomorphism of $] 0, \infty[\times] 0, \infty[$ onto $] 0,1[\times] 0, \pi[$ inverted by

$$
v=\left(\frac{1-r^{2}}{1+2 r \cos \omega+r^{2}}\right)^{1 / 2}, w=\frac{r \sin \omega}{1+2 r \cos \omega+r^{2}} .
$$

Besides

$$
v d v d w=\left(1+2 r \cos \omega+r^{2}\right)^{-2} r d r d \omega .
$$

As usual let $\theta$ denote the Cartan involution of $\mathfrak{g}$. To compute the Iwasawa decomposition of $\bar{n}=\exp (v V+w W)$ it suffices to work in the Lie subalgebra of $\mathfrak{g}$ generated by $V, W, \theta V$ and $\theta W$, a method known as $S U(2,1)$-reduction since this subalgebra corresponds to a Lie subgroup of $G$ isomorphic to $S U(2,1)$. By [27], Chapter IX, Theorem 3.8 we have (remembering our choice (3.44) of the norm and $\|V\|=\|W\|=\sqrt{2}$ )

$$
\begin{aligned}
e^{-2<\rho, H(\bar{n})>} & =\left(\left(1+v^{2}\right)^{2}+4 w^{2}\right)^{-(p / 2)-q} \\
& =2^{-p-2 q}\left(1+2 r \cos \omega+r^{2}\right)^{(p / 2)+q}
\end{aligned}
$$

and the integral formula follows, with $C^{\prime}$ given by the case $f=1$.

(ii) For $q=1$ the group Ad $M$ acts transitively on the unit sphere of $\mathfrak{g}_{-\alpha}$ by Kostant's theorem and trivially on $\mathfrak{g}_{-2 \alpha}$. The integral (B.3) now runs over $v>0$ and $w \in \mathbb{R}$ and the change $(v, w) \mapsto(r, \omega)$ is a diffeomorphism of (] $0, \infty[\times \mathbb{R}) \backslash([1, \infty[\times\{0\})$ onto $] 0,1[\times]-\pi, \pi[$. The result follows as above.

Lemma B.5 Given $x, y>0$ let $\varphi$ be continuous on $[|x-y|, x+y]$. Then, for all rank one spaces,

$$
\int_{K} \varphi(\|Z(x H, k \cdot y H)\|) d k=\int_{|x-y|}^{x+y} \varphi(z) b(x, y, z) d z
$$

with

$$
\begin{aligned}
b(x, y, z)=\frac{2^{n-3} \Gamma\left(\frac{n}{2}\right)}{\Gamma\left(\frac{n-1}{2}\right) \Gamma\left(\frac{1}{2}\right)} \frac{(\operatorname{ch} x \operatorname{ch} y \operatorname{ch} z)^{(p / 2)-1}}{(\operatorname{sh} x \operatorname{sh} y)^{n-2}} \times & \\
\times & \operatorname{sh} z(\operatorname{ch} z)^{q} B^{(n-3) / 2}{ }_{2} F_{1}\left(1-\frac{q}{2}, \frac{q}{2} ; \frac{n-1}{2} ; B\right)
\end{aligned}
$$

and

$$
\begin{aligned}
B= & \frac{1}{\operatorname{ch} x \operatorname{ch} y \operatorname{ch} z} \times \\
& \times \operatorname{sh}\left(\frac{x+y+z}{2}\right) \operatorname{sh}\left(\frac{x+y-z}{2}\right) \operatorname{sh}\left(\frac{x-y+z}{2}\right) \operatorname{sh}\left(\frac{-x+y+z}{2}\right)
\end{aligned}
$$

One has $b(x, y, z)>0$ for $x, y>0$ and $|x-y|<z<x+y$. 
Proof. For $q=0$ the hypergeometric factor is 1 and the result is given by Lemma B.3, where $n=p+1$.

We shall prove the lemma for $q>1$; the case $q=1$ is similar with minor changes.

(i) Since the function $k \mapsto z=\|Z(x H, k \cdot y H)\|$ is $M$-bi-invariant we only need to compute it, by the previous lemma, for $k=k(r, \omega)$. In order to find $z \geq 0$ such that

$$
e^{x H} k(r, \omega) e^{y H}=k^{\prime} e^{z H} k^{\prime \prime}
$$

for some $k^{\prime}, k^{\prime \prime} \in K$, we use $S U(2,1)$-reduction again. By [27] Chapter. IX, Th. 3.1, the Lie subalgebra of $\mathfrak{g}$ generated by $V, W, \theta V$ and $\theta W$ contains $H$ and is isomorphic to $s u(2,1)$. Under this isomorphism $H, V, W$ respectively correspond to

$$
H_{0}=\left(\begin{array}{ccc}
0 & 0 & 1 \\
0 & 0 & 0 \\
1 & 0 & 0
\end{array}\right), V_{0}=\left(\begin{array}{ccc}
0 & 1 & 0 \\
-1 & 0 & -1 \\
0 & -1 & 0
\end{array}\right), W_{0}=\left(\begin{array}{ccc}
i & 0 & i \\
0 & 0 & 0 \\
-i & 0 & -i
\end{array}\right) .
$$

All computations can now be performed in $S U(2,1)$ with the maximal compact subgroup $S(U(2) \times U(1))$; we use subscripts 0 for all notions relative to this group. Let $\bar{n}_{0}=k_{0} e^{t H_{0}} n_{0}$ be the Iwasawa decomposition of

$$
\bar{n}_{0}=\exp \left(v V_{0}+w W_{0}\right)=\left(\begin{array}{ccc}
1-\frac{v^{2}}{2}+i w & v & -\frac{v^{2}}{2}+i w \\
-v & 1 & -v \\
\frac{v^{2}}{2}-i w & -v & 1+\frac{v^{2}}{2}-i w
\end{array}\right),
$$

with

$$
k_{0}=\left(\begin{array}{ccc}
a & b & 0 \\
c & d & 0 \\
0 & 0 & u^{-1}
\end{array}\right),\left(\begin{array}{ll}
a & b \\
c & d
\end{array}\right) \in U(2), a d-b c=u,|u|=1 .
$$

Applying $\bar{n}_{0}$ to the (column) vector $\mathbf{v}={ }^{t}(1,0,1)$ it is easily checked, since $H_{0} \mathbf{v}=\mathbf{v}$ and $n_{0} \mathbf{v}=\mathbf{v}$, that

$$
a u=\frac{1-v^{2}+2 i w}{1+v^{2}-2 i w}=r e^{i \omega}
$$

with $\left(1+r e^{i \omega}\right)\left(1+v^{2}-2 i w\right)=2$ as in the previous lemma.

Besides, a look at the matrix element on third column and third row of $e^{x H_{0}} k_{0} e^{y H_{0}}=k_{0}^{\prime} e^{z H_{0}} k_{0}^{\prime \prime}$ gives (with $u^{\prime}, u^{\prime \prime}$ corresponding to $k_{0}^{\prime}, k_{0}^{\prime \prime}$ )

$$
u u^{\prime-1} u^{\prime \prime-1} \operatorname{ch} z=\operatorname{ch} x \operatorname{ch} y+a u \operatorname{sh} x \operatorname{sh} y .
$$

Setting $e^{i \psi}:=u u^{\prime-1} u^{\prime \prime-1}$ we conclude, first in $S U(2,1)$ then in $G$, that $e^{x H} k(r, \omega) e^{y H}=k^{\prime} e^{z H} k^{\prime \prime}$ implies

$$
e^{i \psi} \operatorname{ch} z=\operatorname{ch} x \operatorname{ch} y+r e^{i \omega} \operatorname{sh} x \operatorname{sh} y
$$


for some $\psi \in \mathbb{R}$.

(ii) Keeping $x, y>0$ fixed let us look at the map $(r, \omega) \mapsto(z, \psi)$ defined by (B.4), with $0<r<1,0<\omega<\pi$ and $z \geq 0,-\pi \leq \psi \leq \pi$. Taking the imaginary part we have

$$
\operatorname{ch} z \sin \psi=r \sin \omega \operatorname{sh} x \operatorname{sh} y
$$

thus $\sin \psi>0$ and $0<\psi<\pi$. Taking the modulus, $\operatorname{ch}(x-y)<\operatorname{ch} z<$ $\operatorname{ch}(x+y)$, thus $|x-y|<z<x+y$. With $B$ defined in the lemma it is readily checked that

$$
\begin{aligned}
B & =\frac{(\operatorname{ch}(x+y)-\operatorname{ch} z)(\operatorname{ch} z-\operatorname{ch}(x-y))}{4 \operatorname{ch} x \operatorname{ch} y \operatorname{ch} z} \\
1-2 B & =\frac{\operatorname{ch}^{2} x+\operatorname{ch}^{2} y+\operatorname{ch}^{2} z-1}{2 \operatorname{ch} x \operatorname{ch} y \operatorname{ch} z},
\end{aligned}
$$

thus $0<B<1 / 2$. Furthermore (B.4) implies $\left|e^{i \psi} \operatorname{ch} z-\operatorname{ch} x \operatorname{ch} y\right|^{2}=$ $r^{2} \operatorname{sh}^{2} x \operatorname{sh}^{2} y$, that is

$$
2 \operatorname{ch} x \operatorname{ch} y \operatorname{ch} z(\cos \psi-1+2 B)=\left(1-r^{2}\right) \operatorname{sh}^{2} x \operatorname{sh}^{2} y .
$$

Therefore $\cos \psi>1-2 B>0$ and we conclude that $(r, \omega) \mapsto(z, \psi)$ is a diffeomorphism of $] 0,1[\times] 0, \pi[$ onto the open set defined by

$$
|x-y|<z<x+y, 0<\psi<\pi / 2, \cos \psi>1-2 B(x, y, z) .
$$

By Lemma B.4 the integral $I=\int_{K} \varphi(\|Z(x H, k \cdot y H)\|) d k$ is

$$
I=C \int_{0}^{\pi} \varphi(z)\left(1-r^{2}\right)^{(p / 2)-1}(r \sin \omega)^{q-1} r d r d \omega
$$

with $z=z(r, \omega)$ given by (B.4) and $C$ by the previous lemma. Using (B.5), (B.8) and $\operatorname{sh} z \operatorname{ch} z d z d \psi=\operatorname{sh}^{2} x \operatorname{sh}^{2} y r d r d \omega$ the integral becomes

$$
\begin{aligned}
I & =C \frac{(2 \operatorname{ch} x \operatorname{ch} y)^{(p / 2)-1}}{(\operatorname{sh} x \operatorname{sh} y)^{n-2}} \times \\
& \times \int \varphi(z)(\operatorname{ch} z)^{(p / 2)-1+q} \operatorname{sh} z d z \int(\cos \psi-1+2 B)^{(p / 2)-1}(\sin \psi)^{q-1} d \psi .
\end{aligned}
$$

Considering the domain of $\psi$ for a given $z$ it is now natural to introduce a variable $t$, running over $] 0,1[$, such that

$$
\cos \psi=1-2 t B .
$$

Then $\sin ^{2} \psi=4 t B(1-t B)$ and the integral with respect to $\psi$ becomes

$$
\begin{aligned}
& 2^{(p / 2)+q-2} B^{(n-3) / 2} \int_{0}^{1} t^{(q / 2)-1}(1-t)^{(p / 2)-1}(1-t B)^{(q / 2)-1} d t= \\
& =2^{(p / 2)+q-2} \frac{\Gamma(p / 2) \Gamma(q / 2)}{\Gamma((n-1) / 2)} B^{(n-3) / 2}{ }_{2} F_{1}\left(1-\frac{q}{2}, \frac{q}{2} ; \frac{n-1}{2} ; B\right)
\end{aligned}
$$


by Euler's integral representation of the hypergeometric function. Since $0<$ $B<1 / 2$ the left-hand side is strictly positive. This implies the lemma, with $b(x, y, z)>0$ for $|x-y|<z<x+y$.

Remarks. (a) Similar computations appear in the study of generalized translation operators by Flensted-Jensen and Koornwinder ([22], or [34] §7.1) in the more general framework of Jacobi functions.

(b) The easy Lemma B.2 may be viewed as a flat limit of Lemma B.5. Indeed on the left-hand side of the integral formula $\varepsilon^{-1} Z(\varepsilon X, \varepsilon Y)$ tends to $X+Y$ as $\varepsilon$ tends to 0 whereas, replacing $x, y, z$ by $\varepsilon x, \varepsilon y, \varepsilon z$ respectively in the righthand side, $B$ tends to 0 , the hypergeometric factor to 1 and $\varepsilon b(\varepsilon x, \varepsilon y, \varepsilon z)$ to $a(x, y, z)$.

Proof of Theorem 3.23. We now combine Lemmas B.2 and B.5. The function

$$
\begin{aligned}
& \frac{b}{a}(x, y, z)=\left(\frac{\operatorname{ch} z}{\operatorname{ch} x \operatorname{ch} y}\right)^{q / 2} \frac{\sigma(z)}{(\sigma(x) \sigma(y))^{n-2}}{ }_{2} F_{1}\left(1-\frac{q}{2}, \frac{q}{2} ; \frac{n-1}{2} ; B\right) \times \\
\times & \left(\sigma\left(\frac{x+y+z}{2}\right) \sigma\left(\frac{x+y-z}{2}\right) \sigma\left(\frac{x-y+z}{2}\right) \sigma\left(\frac{-x+y+z}{2}\right)\right)^{(n-3) / 2}
\end{aligned}
$$

(where $\sigma(t)=\operatorname{sh} t / t$ and $B$ is defined in the previous lemma) is continuous on the set of $(x, y, z) \in \mathbb{R}^{3}$ such that $|x-y| \leq z \leq x+y$. Indeed $0 \leq B<1 / 2$ in this domain by (B.6)(B.7), and the hypergeometric factor is continuous. Taking $\varphi$ continuous on $[|x-y|, x+y]$ we may therefore replace $\varphi(z)$ by $\varphi(z)(b / a)(x, y, z)$ in Lemma B.2, whence

$$
\begin{aligned}
\int_{K} \varphi(\|Z(x H, k \cdot y H)\|) d k & =\int_{|x-y|}^{x+y} \varphi(z) \frac{b}{a}(x, y, z) a(x, y, z) d z \\
& =\int_{K} \varphi(\|x H+k \cdot y H\|) \frac{b}{a}(x, y,\|x H+k \cdot y H\|) d k .
\end{aligned}
$$

Our claim follows, as explained in the outline 3.7.1, with

$$
e(X, Y)=\frac{j(X) j(Y)}{j(X+Y)} \frac{b}{a}(\|X\|,\|Y\|,\|X+Y\|) .
$$

We now specialize to $j=J^{1 / 2}$. In the rank one case any $X \in \mathfrak{p}$ may be written as $X=k \cdot x H$ with $x \geq 0, k \in K$. Since $\alpha(x H)=x=\|X\|$ the Jacobian of Exp is

$$
J(X)=J(x H)=\left(\frac{\operatorname{sh} x}{x}\right)^{p}\left(\frac{\operatorname{sh} 2 x}{2 x}\right)^{q}=\sigma(x)^{n-1}(\operatorname{ch} x)^{q} .
$$

For $j=J^{1 / 2}$ we thus obtain

$$
e(X, Y)=A(x, y, z)^{(n-3) / 2}{ }_{2} F_{1}\left(1-\frac{q}{2}, \frac{q}{2} ; \frac{n-1}{2} ; B(x, y, z)\right)
$$


as claimed, with $x=\|X\|, y=\|Y\|, z=\|X+Y\|$. Clearly $e(k \cdot X, k \cdot Y)=$ $e(X, Y)$ for $k \in K$.

Besides, $A$ and $B$ are analytic functions of $(x, y, z) \in \mathbb{R}^{3}$, even with respect to each variable, therefore define analytic functions of $\left(x^{2}, y^{2}, z^{2}\right)$. Thus $A(\|X\|,\|Y\|,\|X+Y\|)$ is an analytic function of $(X, Y) \in \mathfrak{p} \times \mathfrak{p}$ and the same holds for $B$. Since $|x-y| \leq z \leq x+y$ for the chosen values we have $0 \leq B(x, y, z)<1 / 2$, which implies analyticity of the hypergeometric factor too. The theorem now follows from Proposition 3.16. 


\section{Bibliography}

[1] AlBERT, L., HARINCK, P. and TOROSSIAN, C., Solution non universelle pour le problème $K V$ 78, J. Lie Theory 18 (2008), 617-626; arXiv:0802.2049 (2008).

[2] Alekseev, A., Enriquez, B. and TOROSsian, C., Drinfeld associators, braid groups and explicit solutions of the Kashiwara-Vergne equations, Publ. Math. I.H.E.S. 112 (2010), 146-189; arXiv:0903.4067 (2009).

[3] AlEKSEeV, A. and MEINREnKEn, E., Poisson geometry and the Kashiwara-Vergne conjecture, C. R. Acad. Sci. Paris, Série I, 335 (2002), 723-728.

[4] ALEKSEEV, A. and MEINRENKEN, E., Lie theory and the Chern-Weil homomorphism, Ann. Sci. École Norm. Sup. 38 (2005), 303-338.

[5] AleKSEeV, A. and MeInREnKEN, E., On the Kashiwara-Vergne conjecture, Invent. Math. 164 (2006), 615-634.

[6] ALEKSEEV, A. and PETRACCI, E., Low order terms of the CampbellHausdorff series and the Kashiwara-Vergne conjecture, J. Lie Theory 16 (2006), 531-538; arXiv:math.QA/0508077 (2005).

[7] ALEKSEeV, A. and TOROSSIAN, C., The Kashiwara-Vergne conjecture and Drinfeld's associators, Ann. of Maths. 175 (2012), 415-463; arXiv:0802.4300 (2008).

[8] ALEKSEEV, A. and TOROSSIAN, C., On triviality of the KashiwaraVergne problem for quadratic Lie algebras, C. R. Acad. Sci. Paris, Série I, 347 (2009), 1231-1236; arXiv:0909.3743 (2009).

[9] ANDLER, M., SAHI, S. and TOROSSIAN, C., Convolution of invariant distributions: proof of the Kashiwara-Vergne conjecture, Lett. Math. Phys. 69 (2004), 177-203; arXiv: math.QA/0104100 (2001).

[10] BENOIST, Y., Sur l'algèbre des opérateurs différentiels invariants sur un espace symétrique nilpotent, C. R. Acad. Sci. Paris, Série A, 295 (1982), 59-62. 
[11] BONFIGLIOLI, A. and FULCI, R., Topics in noncommutative algebra: the theorem of Campbell, Baker, Hausdorff and Dynkin, Lecture Notes in Math. 2034, Springer 2012.

[12] BURGUNDER, E., Eulerian idempotent and Kashiwara-Vergne conjecture, Ann. Inst. Fourier 58 (2008), 1153-1184; arXiv:math.QA/0612548 (2006).

[13] CASAS, F. and MURUA, A., An efficient algorithm for computing the Baker-Campbell-Hausdorff series and some of its applications, J. Math. Physics 50 (2009), 033513; arXiv:0810. 2656 (2008).

[14] CATTANEO, A., ROSSI, C. and TOROSSIAN, C., Biquantization of symmetric pairs and the quantum shift, J. Geom. Phys. 74 (2013), 211250; arXiv:1105.5973v2 (2012).

[15] CATTANEO, A. and TOROSSIAN, C., Quantification pour les paires symétriques et diagrammes de Kontsevich, Ann. Sci. École Norm. Sup.41 (2008), 789-854; arXiv:math.RT/0609693 (2006).

[16] DOOLEY, A.H. and RICE, J.W., On contractions of semi-simple Lie groups, Trans. Amer. Math. Soc. 289 (1985), 185-202.

[17] DOOLEY, A.H., Orbital convolutions, wrapping maps and e-functions, Proc. Centre for Math. Anal. and Appl., National Research Symposium on Geometric Analysis and Applications, 39 (2001), 42-49.

[18] DUFLO, M., Opérateurs différentiels bi-invariants sur un groupe de Lie, Ann. Sci. École Norm. Sup. 10 (1977), 265-288.

[19] DUFLO, M., Opérateurs différentiels invariants sur un espace symétrique, C. R. Acad. Sci. Paris, Série I, Math. 289 (1979), 135-137.

[20] DUISTERMAAT, J.J., On the similarity between the Iwasawa projection and the diagonal part, Mém. Soc. Math. France n 15 (1984), 129-138.

[21] ETINGOF, P. and SCHIFFMAN, O., Lectures on quantum groups, International Press, Cambridge 1998.

[22] FLENSTED-JENSEN, M. and KOORNWINDER, T., The convolution structure for Jacobi function expansions, Ark. Mat. 10 (1973), 245-262.

[23] GRACZYK, P. and SAWYER, P., On the kernel of the product formula on symmetric spaces, J. Geom. Anal. 14 (2004), 653-672.

[24] GRAY, A. and WILLMORE, T.J., Mean-value theorems for Riemannian manifolds, Proc. Royal Soc. Edinburgh 92A (1982), 343-364.

[25] GUILlEMIN, V. and STERNBERG, S., Symplectic techniques in physics, Cambridge University Press, Cambridge 1984. 
[26] HARISH-CHANDRA, Invariant eigendistributions on a semi-simple Lie group, Trans. Amer. Math. Soc. 119 (1965), 457-508.

[27] HELGASON, S., Differential geometry, Lie groups and symmetric spaces, Academic Press, New-York 1978.

[28] HELGASON, S., Groups and geometric analysis, Academic Press, Orlando 1984.

[29] HELGASON, S., Geometric analysis on symmetric spaces, second edition, American Math. Society 2008.

[30] KASHIWARA, M. and VERGNE, M., The Campbell-Hausdorff formula and invariant hyperfunctions, Invent. Math. 47 (1978), 249-272.

[31] KOBAYASHI, S. and NOMIZU, K., Foundations of differential geometry, volume II, Interscience Publishers 1969.

[32] KONTSEVICH, M., Deformation quantization of Poisson manifolds, Lett. Math. Phys. 66 (2003), 157-216; arXiv:math.QA/9709040 (1997).

[33] KOORNWINDER, T., A new proof of a Paley-Wiener type theorem for the Jacobi transform, Arkiv för Mat. 13 (1975), 145-159.

[34] KOORNWINDER, T., Jacobi functions and analysis on noncompact semisimple Lie groups, in Special functions: group theoretical aspects and applications (R. Askey ed.), D. Reidel 1984.

[35] KOORNWINDER, T., Invariant differential operators on nonreductive homogeneous spaces, arXiv:math/0008116 (2000).

[36] LICHNEROWICZ, A., Opérateurs différentiels invariants sur un espace homogène, Ann. Sci. École Norm. Sup. 81 (1964), 341-385.

[37] LOOS, O., Symmetric spaces, volume I, Benjamin, New-York 1969.

[38] MINEMURA, K., Invariant differential operators and spherical sections on a homogeneous vector bundle, Tokyo J. Math. 15 (1992), 231-245.

[39] ORLOFF, J., Invariant Radon transforms on a symmetric space, Trans. Amer. Math. Soc. 318 (1990), 581-600.

[40] RAÏS, M., Solutions élémentaires des opérateurs différentiels biinvariants sur un groupe de Lie nilpotent, C. R. Acad. Sci. Paris, Série A, 273 (1971), 495-498.

[41] ROSSI, C., On the compatibility between cup products, the AlekseevTorossian connection and the Kashiwara-Vergne conjecture, C. R. Acad. Sci. Paris, Série I, 350 (2012), 823-826 and 871-874; arXiv: 1205.7029v3 (2012) 
[42] ROUVIÈRE, F., Démonstration de la conjecture de Kashiwara-Vergne pour l'algèbre sl(2), C. R. Acad. Sci. Paris, Série I, 292 (1981), 657-660.

[43] ROUVIÈRE, F., Espaces symétriques et méthode de Kashiwara-Vergne, Ann. Sci. École Norm. Sup. 19 (1986), 553-581.

[44] ROUVIÈRE, F., Invariant analysis and contractions of symmetric spaces I, Compositio Math. 73 (1990), 241-270.

[45] ROUVIÈRE, F., Invariant analysis and contractions of symmetric spaces II, Compositio Math. 80 (1991), 111-136.

[46] ROUVIÈRE, F., Une propriété de symétrie des espaces symétriques, C. R. Acad. Sci. Paris, Série I, 313 (1991), 5-8.

[47] ROUVIÈRE, F., Fibrés en droites sur un espace symétrique et analyse invariante, J. Funct. Anal. 124 (1994), 263-291.

[48] ROUVIÈRE, F., «Trivialité» du problème de Kashiwara-Vergne pour les algèbres de Lie résolubles, C. R. Acad. Sci. Paris, Série I, 348 (2010), 739-742.

[49] ROUVIÈRE, F., Mean value theorems on symmetric spaces, Contemporary Mathematics 598 (2013), 209-219.

[50] SHIMURA, G., Invariant differential operators on hermitian symmetric spaces, Ann. of Math. 132 (1990), 237-272.

[51] THOMPSON, R. C., Proof of a conjectured exponential formula, Linear and Multilinear Algebra 19 (1986), 187-197.

[52] TOROSSIAN, C., Opérateurs différentiels invariants sur les espaces symétriques, II: L’homomorphisme de Harish-Chandra généralisé, J. Funct. Anal. 117 (1993), 174-214.

[53] TOROSSIAN, C., L'homomorphisme de Harish-Chandra pour les paires symétriques orthogonales et parties radiales des opérateurs différentiels invariants sur les espaces symétriques, Bull. Soc. math. France 126 (1998), 295-354.

[54] TOROSSIAN, C., Sur la conjecture combinatoire de Kashiwara-Vergne, J. Lie Theory 12 (2002), 597-616.

[55] TOROSSIAN, C., Méthode de Kashiwara-Vergne-Rouvière pour certains espaces symétriques, in Non-commutative harmonic analysis, Birkhaüser, Progress in Math. vol. 220 (2004), 459-486; arXiv:math.QA/0202217 (2002).

[56] TOROSSIAN, C., Paires symétriques orthogonales et isomorphisme de Rouvière, J. Lie Theory 15 (2005), 79-87. 
[57] TOROSSIAN, C., La conjecture de Kashiwara-Vergne [d'après Alekseev et Meinrenken], Séminaire Bourbaki n 980, Astérisque 317 (2008), 441465 .

[58] VARADARAJAN, V.S., Lie groups, Lie algebras, and their representations, Springer 1984.

[59] VERGNE, M., Le centre de l'algèbre enveloppante et la formule de Campbell-Hausdorff, C. R. Acad. Sci. Paris, Série I, 329 (1999), 767772 .

[60] WALLACH, N., Harmonic analysis on homogeneous spaces, Marcel Dekker 1973.

[61] WOLF, J.A., Spaces of constant curvature, third edition, Publish or Perish 1974. 


\section{Index}

$A(X, Y), 121$

Abel transform, 93

associator, 44

Bessel function, 61, 78, 86, 97

$c(\lambda), 85,96$

$C(X, Y), 121$

$c(X, Y), 82,125$

Campbell-Hausdorff

$e$-function, 130

formula, 3

contraction, 113

convolution

on homogeneous space, 47

on line bundle, 51

$\mathbb{D}(S), 55$

$\mathbb{D}(S), 49$

density, 50

divergence, 30

Drinfeld associator, 44

Duflo isomorphism, 3

generalized, 71, 92

$e_{\infty}, 85$

$e(X, Y), 130$

$e_{\chi}(X, Y), 146$

$e$-function

construction of, 130

definition of, 57

expansion of, 152, 160

structure of, 140

symmetry of, 145

expansion of $e, e_{\chi}, 152,160$

of $\Phi, 151,158$

of mean value operator, 79

of spherical function, 79

of $Z, h, A, C, 150,155$

$F(X, Y), G(X, Y), 7,121$

Fischer product, 64

free Lie algebra, 10

$\mathfrak{h}_{*}, \mathrm{xvi}$

$h(X, Y), 117$

hyperbolic space, $69,83,98,103,113$,

153

invariant differential operator

and convolution, 49

and $e$-function, 65, 133

transfer of, 55

invariant measure, 55

isotropic symmetric space, 73

Iwasawa projection, 85

$J, 55$

$j, 2,54$

$(j, \Phi)$-function, 130

$(j, F, G)$-function, 130

$(j, \Phi, \chi)$-function, 146

Jacobian

"Jacobian" $\mathcal{J}, 31$

of Exp, xvii

of exp, xvi

Kashiwara-Vergne

conjecture, 10

first equation, $7,120,137$ 
method, 3

second equation, 9, 137

$L_{\chi}, 50$

$\mathfrak{l}_{2}, 10$

Laplace-Beltrami operator, 68

Lie series, 10, 114

line bundle, 50, 105, 146

mean value operator

general case, 78

on isotropic space, 74

$\mathcal{O}, 116$

$\Omega, 122$

$\Omega_{1}, 57$

orthogonal projection, 85

$P_{e}\left(X, \partial_{X}\right), 65$

$P \times Q, 66$

$\pi, 85$

$\Phi(X, Y), 122$

$\psi_{X}, 82,136$

Pizzetti's formula, 76

quadratic Lie algebra, 40

radial component, 91, 165

Riemannian symmetric space, 84 of rank one, 98

$\mathfrak{s}^{\prime}, 54$

scaling, 28

semi-invariant measure, 128, 145

solvable

Lie algebra, 40

symmetric space, 143

special line bundle, 106, 148

special symmetric space, 57, 59, 143

spherical function, 60,85

expansion of, 79

rank one case, 104

spherical transform, 92

strongly symmetric, 142

SU $(2,1)$-reduction, 173

symmetry of $e, 145$

of $e_{\chi}, 148$

of the $\mathrm{KV}$ problem, 20

tangential automorphism, 27

tangential derivation, 26

test function, $\mathrm{xv}$

transfer, 2, 54, 105

$\mathcal{U}, 115$

$V(X, Y), 3$

$Z(X, Y), 58,117$ 